\title{
Using Mobility for Agility: Enhancing Wireless Networks with Aerial Access Nodes and User Involvement
}

\author{
by \\ Irem Bor-Yaliniz, M.Sc. \\ A Dissertation Proposal in partial fulfillment \\ of the requirements for the degree of
}

Doctor of Philosophy in Electrical and Computer Engineering

The Ottawa-Carleton Institute for

Electrical and Computer Engineering (OCIECE)

Department of Systems and Computer Engineering

Carleton University

Ottawa, Ontario

March, 2020

(C)Copyright

Irem Bor-Yaliniz, 2020 


\section{Abstract}

Considering numerous futuristic applications that will be enabled by wireless networks, one may wonder the essence of the next evolution in wireless networks. Recently, concepts and technologies such as polar codes, wireless network virtualization, millimeter wave communications, and non-orthogonal multiple access have emerged as new enablers. They provide promising solutions for significant problems by enhancing capacity, flexibility, and spectrum usage. However, they cannot address precisely the most needed new capability to handle diverse applications without relying on to gross over-engineering: Agility. This study proposes a new solution to dramatically improve agility without leaning on overengineering: Using mobility for agility, where the inherent support of wireless networks for user terminal mobility is used to support the mobility of access points. In addition, we propose to influence the user demand in space and time to flexibly shape the network from both sink and source perspectives.

We propose the spatial network configuration (SNC) scheme, which utilizes dronebase-stations (drone-BSs) to re-configure topology of access points, and user-in-the-loop (UIL) to influence demand of users. Drone-BSs are shifting paradigms of heterogeneous wireless networks by providing radically flexible deployment opportunities. On the other hand, their limited endurance and potential high cost increase the importance of utilizing drone-BSs efficiently. Therefore, we thoroughly investigate efficient utilization of droneBSs from placement methods to refinements on air-to-ground channel modeling. To further exploit drone-BSs, we influence locations of users via the UIL method, which aims at influencing mobility of the users by offering incentives. Finally, we investigate the SNC scheme with a holistic approach and propose the drone management framework. We show that the integration methods severely affect the network performance when wireless access virtualization is applied. Furthermore, we survey the latest developments in 3GPP 5G Release-16 standardization to discuss the capabilities and shortcomings of current and 
prospective wireless networks to integrate drone-BSs efficiently. All in all, this study shows that it is time to re-imagine future wireless networks in three dimensions. 
To my mother, Gözen,

and

To my husband, Gökhan,

who have tirelessly supported me through thick and thin.... 


\section{Acknowledgments}

I am deeply grateful for my PhD studies, which enhanced my life both professionally and personally, with the help of many amazing people, among which certainly my supervisor Prof. Halim Yanikomeroglu comes first. His generous support, strong professionalism, exemplary attitudes, profound knowledge, and patience have guided this thesis for the more visionary and myself for the better.

Huawei Technologies Canada research and development team has provided fundamental support, the most valuable discussions, and nonesuch guidance for the completion of this thesis, specially Dr. Gamini Senarath, Dr. Mohamed Salem, Dr. Hang Zhang, Dr. Ngoc Dao, Dr. Xu Li, Bill Gage, Dr. Jaya Rao, and Arashmid Akhavain. I would also like to mention Dr. Gamini Senarath for his patience in turning me from a novice professional to a seasoned one, and for his guidance that is beyond the limits of this thesis; for generously sharing his profound wisdom on life, and for teaching how to cope with difficult times. I would also like to specifically mention Dr. Mohamed Salem for many valuable discussions, and being an exemplary colleague with his hard work and professionalism. Knowledge and talent of Dr. Amr El-Keyi have laid the foundations for this thesis. I cannot thank him enough for his valuable time spent on this thesis, and for his patience on reading the first conference paper draft that I have ever written, and giving me such valuable feedback that I could write many others on my own later; words are not enough to express my gratitude for him to teach me how to fish. I would like to mention Dr. Sebastian Szyszkowic for his help with channel modelling studies. His help provided me with the much needed kick-start. I would like to thank all my colleagues, with whom I collaborated at different capacities, for their knowledge, support, hard work and patience, specifically, Prof. Abbas Yongacoglu, Dr. Sergey Andreev, Dr. Elham Kalantari, Dr. Faraj Lagum, Margarita Gapeyenko, Dr. Mohammad Mozaffari, Dr. Bulent Tavli, Dr. Salman Durrani, Dr. Gunes Karabulut-Kurt, and Dr. Jalal Khamse-Ashari. I would like to thank Dr. Rozita Rastchi and 
Dr. Yasser Fouad for guiding me during the early times of my $\mathrm{PhD}$, when I needed it the most. Their presence has given me courage, and lighted my way. I am grateful for many enriching discussions on research, course work, and life with our wonderful research team, as well as Dr. Hamid Saber and Dr. Zeeshan Shakir. I would like to thank Dr. Chengchao Liang, and Dr. Monirosharieh Vameghestahbanati for their warm friendship and inspiring ideas.

Last but not the least, I would like to express my gratitude for my friends and family. Thankfully, the list of my friends would make a long one, and it is hard to express my gratitude for each dear friend. I would like to specifically mention Ebru, who is far away but the closest, who is my sister from another mother, whose presence is uniquely wonderful. I should extend a very special thanks to my uncle, Kâni Bor, who made the very beginning of this journey possible by supporting me during my undergraduate studies at many capacities. Although his presence and love would be more than enough to find encouragement, he have always gone above and beyond to support me generously; from the times when I was a sleepless baby till today when I am sleepless because of my baby. I am grateful for my husband, Gökhan, who respected and supported my studies, who bear with me during sleepless nights, as well as ups and downs of life. I am grateful for my mother, Gözen, who made me who I am today, supported me with her endless compassion and love, and gave me courage to go on when I wanted to give up. I would like to thank to my sister, İpek and my grandmother, Aynur, for making my belly hurt from laughing and cheering me up when I am vulnerable. And finally, I would like to acknowledge my son, Atlas, for being my hardest yet the most joyful challenge in completing my thesis. 


\section{Table of Contents}

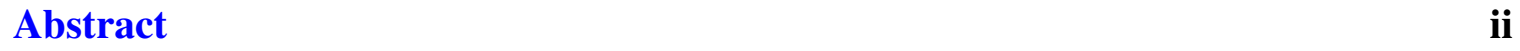

Acknowledgments $\quad$ v

Table of Contents vii

List of Tables $\quad$ xii

List of Figures $\quad$ xiv

Nomenclature $\quad$ xix

1 Introduction 1

1.1 List of Publications . . . . . . . . . . . . . . . . 8

1.1.1 Published Journal Papers — included in the thesis . . . . . . . 8

1.1.2 Published Journal Papers — not included in the thesis . . . . . . 9

1.1.3 Submitted Journal Papers — not included in the thesis . . . . . . 9

1.1.4 Published Conference Papers — included in the thesis . . . . . . 9

1.1.5 Published Conference Papers — not included in the thesis . . . . . 10 
1.1.6 Patent Filings - not included in the thesis $\ldots \ldots \ldots$

2 3-D Drone-BS Placement $\quad 12$

2.1 Efficient 3-D Placement . . . . . . . . . . . . . . . . . . . . . 14

$2.1 .1 \quad$ System Model . . . . . . . . . . . . . . . . . . . . 16

2.1.2 Efficient 3-D Placement of a Drone-BS . . . . . . . . . . . . 17

2.1.3 Numerical Results of Efficient 3-D Placement Method . . . . . . . 21

2.2 Efficient 3-D Placement for a MmWave-drone-BS . . . . . . . . . . . . 25

2.2.1 System Model for MmWave-drone-BS Placement . . . . . . . . . . 27

2.2.2 MmWave-drone-BS Deployment Method . . . . . . . . . . . 30

2.2.3 Numerical Results and Discussions _ . . . . . . . . . . . . 33

2.3 Environment-Aware Drone-Base-Station Placements in Modern Metropolitans . . . . . . . . . . . . . . . . . 37

2.3.1 Environment For Channel Modeling . . . . . . . . . . . . . . . 38

2.3.2 Channel Models for Low and Ultra-low Altitude Platforms . . . . 39

2.3.3 Simulation Results . . . . . . . . . . . . . . . . . . . . . 42

2.4 Summary and Discussions $\ldots \ldots \ldots \ldots$. . . . . . . . . . 47

3 User-Centric Spatial Network Configuration 49

3.1 User Involvement Models . . . . . . . . . . . . . . . . . . . . . . . 50

3.2 System Model for Evaluations $\ldots \ldots \ldots \ldots$

3.2.1 User-in-the-loop Model . . . . . . . . . . . . . . . . . . . . . 54

3.3 Uncoordinated Spatial Network Configuration . . . . . . . . . . . . 56 
3.3.1 3-D Placement of a Drone-BS . . . . . . . . . . . . 57

3.4 Incentive Design for User-in-the-loop . . . . . . . . . . . . . . 60

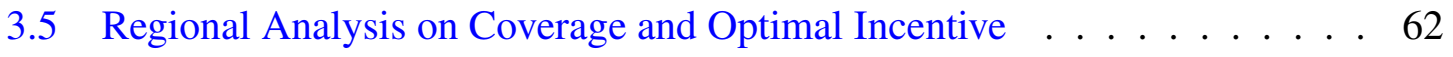

3.5.1 Extended Coverage of a BS in a System with UIL . . . . . . . . . . 62

3.5.2 Optimal Regional Incentive . . . . . . . . . . . . 63

3.6 Joint Spatial Network Configuration . . . . . . . . . . . . . . 65

3.7 Semi-Joint Spatial Network Configuration . . . . . . . . . . . . . 72

3.8 Simulations and Results . . . . . . . . . . . . . . . . 76

3.9 Summary and Discussions . . . . . . . . . . . . . . . 79

\section{Integration of Aerial Nodes $\quad 82$}

4.1 The New Frontier in RAN Heterogeneity: Multi-Tier Drone-Cells . . . . . 84

4.1.1 Descriptions and Opportunities . . . . . . . . . . . . . . . 84

4.1.2 Challenges of Drone-cells . . . . . . . . . . . . . . 87

4.1.3 Challenges of Multi-tier Drone-cell Networks . . . . . . . . . . . . 89

4.2 The Drone-cell Management Framework . . . . . . . . . . . . . . . 90

4.2.1 Enabling Technologies for DMF . . . . . . . . . . . . . 91

4.2.2 A Case Study: 3-D Placement of a Drone-cell via DMF . . . . . . 96

4.3 Drone-BS Integration with Virtual Wireless Access . . . . . . . . . 101

4.3 .1 System Model . . . . . . . . . . . . . . 103

4.3.2 Optimal TP Grouping and Drone-BS Integration strategies . . . . . 105

4.3.3 Reinforcement Learning Based Integration of a Drone-BS . . . . 109 
4.3.4 Simulation Results and Discussions . . . . . . . . . . . . 113

4.4 Summary and Discussions . . . . . . . . . . . . . 116

5 Is 5G Ready for Drones? $\quad 118$

5.1 Drones and Mobile Networks . . . . . . . . . . . . . . . . . 119

5.1.1 Mobile-Enabled Drones . . . . . . . . . . . . . . 119

5.1 .2 Wireless Infrastructure Drones . . . . . . . . . . . . . . 121

5.2 Progress of 5G Standardization and What It Means for Drones . . . . . . 122

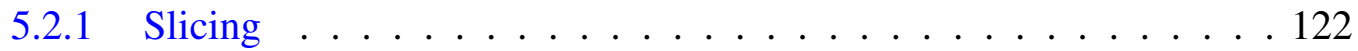

5.2.2 Standardization for MEDs in 5G RAN . . . . . . . . . . . . . . 124

5.2.3 Architectural Roles in 5G RAN . . . . . . . . . . . . . . . 124

5.2.4 Integrated Access and Backhaul . . . . . . . . . . . . . 132

5.2.5 Licensed/Unlicensed options for MEDs and WIDs . . . . . . . . 133

$5.35 \mathrm{G}$ Core $\ldots \ldots \ldots \ldots \ldots \ldots$

5.45 G Network Management . . . . . . . . . . . . . . . . . 137

5.5 Future of Standardization and Research Directions _ . . . . . . . . . 139

5.5.1 Network Configuration and Slice Design for WIDs . . . . . . . . 139

5.5.2 Support For New Business Models . . . . . . . . . . . . . . . . . . 140

5.5.3 New QoS and KPI Parameters . . . . . . . . . . . . . . 141

5.6 Summary and Discussions . . . . . . . . . . . . . . . . 142

6 Conclusions and Future Work 143 
6.1 Summary and Conclusions . . . . . . . . . . . . . . . 143

6.2 Future Work . . . . . . . . . . . . . . . . . . . . . 144

6.2 .1 User Involved IGASC . . . . . . . . . . . . . . . . . . 145

6.2.2 Self-Adapting and Autonomous IGASC Networks . . . . . . . . 146

6.2 .3 IoT in IGASC . . . . . . . . . . . . . . . . . . 148

6.2.4 Channel modelling for IGASC . . . . . . . . . . . . . . . . . . 148 


\section{List of Tables}

2.1 RF Propagation parameters of different environments . . . . . . . . . . . 22

2.2 Simulation Parameters ...................... 23

2.3 Notation and parameters . . . . . . . . . . . . . . . . 29

2.4 Baseline system parameters . . . . . . . . . . . . . . . 35

2.5 Parameters for ray-tracing and channel models. . . . . . . . . . . . . . 45

2.6 RMSE of propagation models for several building and drone-BS heights, $h_{B}$ and $h$, respectively. . . . . . . . . . . . . . . 46

3.1 Simulation Parameters . . . . . . . . . . . . . . . 77

4.1 An example of categorization of test cases of METIS requiring agility and resilience: An event can fall under one category or multiple categories and each combination may require different solutions. For instance, connectivity requirements in case of an only temporary event (e.g., stadium) may be addressed by over-engineering. Then, expenses of drone-BS operations may be compared to the expenses of over-engineering, including energy and maintenance costs. On the other hand, for both temporary and unexpected events, (e.g. traffic jam), drone-BSs may be utilized opportunistically. For temporary, unexpected and critical operations (e.g., emergency communications) drone-cells can provide much more than revenue, such as saving lives. . . . . . . . . . . . . . . . . . 86

4.2 Various information that can be gathered in the cloud. . . . . . . . . 95 
4.3 Simulation Parameters . . . . . . . . . . . . . . . . . . 114

5.1 QoS requirements for URLLC, eMBB and MED scenarios in 3GPP RAN documents. . . . . . . . . . . . . . . . . . 120 


\section{List of Figures}

2.1 A possible scenario showing the users that are not covered by the eNB. Three potential placements of a drone-BS is highlighted. . . . . . . . 15

$2.2 \Gamma(\alpha)$ versus $\alpha$ for various environments. . . . . . . . . . . . . 21

2.3 Effect of environment on the location and size of the coverage area (circles in the figure) is shown. . . . . . . . . . . . . . . 24

2.4 Mean number of users covered in different environments with 95\% confidence interval. 40 users are distributed uniformly in one macrocell. . . . . 25

2.5 Target scenario with mmWave-drone-BS, users, and blockers. . . . . . . . 27

2.6 Comparison of the optimal altitude results from 3-D placement with theoretical analysis vs. density of blockers, $\lambda . \ldots \ldots \ldots$. . . . . . 34

2.7 Radius, $\mathrm{R},(\mathrm{m})$ vs. density of blockers, $\lambda$, with error bar. . . . . . . . . . 35

2.8 Number of users served by mmWave-drone-BS vs. density of blockers, $\lambda$. . 36

2.9 Path loss patterns for a drone-BS at $50 \mathrm{~m}$ altitude. . . . . . . . . . . 41

2.10 Path loss patterns for a drone-BS at $1000 \mathrm{~m}$ altitude. . . . . . . . . . 41

2.11 Propagation data at $50 \mathrm{~m}$ in comparison to ray-tracing simulations. . . . . 43

2.12 Propagation data at $1000 \mathrm{~m}$ in comparison to ray-tracing simulations. . . . 44

2.13 Number of covered users with PM, UCM, and ray-tracing simulations. . . . 47 
3.1 Description of SNC: Users in region (1) are covered without additional cost. Users in region 2 are within the extended coverage region, therefore, they receive an incentive offer to move towards the coverage area. Users in region 3 cannot be covered even with UIL, and hence do not receive an offer. . . . . . . . . . . . . . . . . . . . 5 54

3.2 Probability of a user accepting to move with respect to incentive (percentage of price discount) and distance: Survey data is fit to $\beta(\tau)$ in (3.2) . . . . 56

3.3 Unit profit vs. incentive for several distances. . . . . . . . . . . . . 61

3.4 Gain from the UIL with respect to regional incentive, $\tau$ is calculated for $R=200, W=2000 \ldots \ldots \ldots \ldots \ldots \ldots$

3.5 Optimal regional incentive is calculated with respect to $d_{u}$ for $R=$ $\{100,200,600\}, W=2000 \ldots \ldots \ldots \ldots \ldots$

3.6 Coverage area of a drone-BS $(\mathcal{R})$, area where the UIL model is utilized $(\mathcal{D})$,

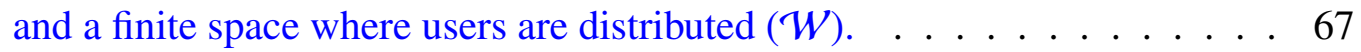

3.7 Illustration of the triangle method on a portion of $\Pi(\tau, d) \ldots \ldots$

3.8 Piece-wise approximation with 3 and 4 breakpoints, and the resulting RMSE values, where $d_{u}=200 \mathrm{~m} \ldots \ldots \ldots$. . . . . . . . . . 74

3.9 A simple example demonstrating the difference of placement and profit with and without SNC framework. Normalized profit is 3 without SNC, and 4.16 with JSNC . . . . . . . . . . . . . . . . . . . . 76

3.10 CDF of the computation time of the proposed methods. . . . . . . . . 78

3.11 CDF of the profit obtained by the proposed methods. . . . . . . . . . 79

3.12 The comparison of the proposed methods based on mean unit profit versus the density of the users. . . . . . . . . . . . . . . . . . . 80 
4.1 Multi-tier drone-cell networks can be used for many scenarios: (1) Providing service to rural areas (macro-drone-cell), 2 Deputizing for a malfunctioning BS (macro-drone-cell), 3 Serving users with high mobility (femto-drone-cell), (4) Assisting a macrocell in case of RAN congestion (pico-drone-cell), 5 Assisting a macrocell in case of core network congestion or malfunctioning (macro-drone-cell), 6 Providing additional resources for temporary events, e.g., concerts and sports events, 7 Providing coverage for temporary blind spots, and 8 Reducing energy dissipation of sensor networks by moving towards them (femto-drone-cell). . . . .

4.2 DMF mechanism and potential business and information model demonstrating partitioning of the traditional MNO into InP (cloud, server, droneBS etc.) and MVNO: (1) Collect and store global data; 2 Process data for network monitoring and creating intelligence; 3 Provide guidance for drone-cell's operation (placement, content to be loaded, access technology, service duration, coverage area, moving patterns); (4 Re-configure the virtual network of MVNO for drone-cell integration by SDN and NFV technologies, e.g., introduce another gateway to handle busy traffic and create new paths among the new and existing functions; 5 Drone-cell assists the network; 6 SP can continue delivering services successfully. . . . . . 94

4.3 Effect of different policies on 3-D placement of a drone-BS. . . . . . . . . 99

4.4 Mean number of users covered by the drone-cell with 3-D placement in different environments. . . . . . . . . . . . . . . . . . 100

4.5 The VWA framework with a drone-BS. . . . . . . . . . . . . . 102

4.6 A generic RL scheme. . . . . . . . . . . . . . . . . . 110

4.7 Grid world representation of the joint drone-BS placement and TP grouping

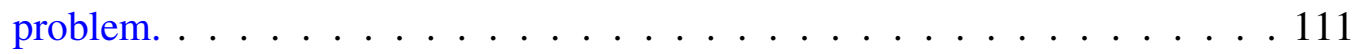

4.8 CDF of average SE and SINR with respect to TP group size. . . . . . . . 113

4.9 CDF of average SE with various integration methods. . . . . . . . . 115 
$4.10 \mathrm{CDF}$ of number of users covered by the drone-BS. . . . . . . . . . . . 116

$4.11 \mathrm{CDF}$ of number of iterations until convergence. . . . . . . . . . . . 117

5.1 Wireless networks with slicing and integration of WIDs: (a) Creation of logical networks from infrastructure pools. Some initial UE-attachment steps are highlighted. (b) Interfaces and service-based 5G-core slices. A WID-specific 5G-core slice may include common and non-shared NFs; the upper WID slice has isolated resources from the gNB slice below. Circle depicts gNB resources reserved to provide backhaul and/or fronthaul to the WID (hard-slicing in RAN). . . . . . . . . . . . . . . . . . . . . 123

5.2 Combined protocol stack architecture and simplified reference point model for licensed/unlicensed WID integration with Drone-SgNB using NR/NR$\mathrm{U}$ radio in EN-DC option 3/3A . . . . . . . . . . . . . . . . . . 125

5.3 Combined protocol stack architecture and simplified reference point model for licensed/unlicensed WID integration with Drone-SgNB using NR/NRU radio in NE-DC option 4/4A. . . . . . . . . . . . . . . . . . . . 126

5.4 Combined protocol stack architecture and simplified reference point model for licensed/unlicensed WID integration with WLAN termination drone in NR-WLAN aggregation. . . . . . . . . . . . . . . . . . . . 127

5.5 Combined protocol stack architecture and simplified reference point model for licensed/unlicensed WID integration with Drone IPsec Access Point in NR-WLAN integration using IPsec tunnel. . . . . . . . . . . . . . . 128

5.6 Architecture option for WIDs where a standalone aerial-gNB with a specific 5G-core-slice for WID-originated traffic (blue slice) is utilized. Interfaces with potential enhancements are marked with "w". . . . . . . . . . 130

5.7 Architecture option for WIDs where a fleet of WIDs with aerial-DUs and an aerial-CU with a specific 5G-core-slice for WID-originated traffic (blue slice) is utilized. Interfaces with potential enhancements are marked with "w". . . . . . . . . . . . . . . . . . . . 130 
5.8 Architecture option for WIDs where Aerial-DUs associated with terrestrial-CUs (no specific 5G-core-slice for WID-originated traffic) is utilized. Interfaces with potential enhancements are marked with “w”. . . . . 131

5.9 Architecture option for WIDs where WIDs are utilized as relay nodes and intermediate IAB nodes. There are at least protocol stack differences between the two types of nodes. . . . . . . . . . . . . . . . 131

5.10 Network management system overview: (a) SBA allows any network management service provider to access another via "request-response" or "subscribe-notify" messages; (b) Overview of network slice provisioning procedures: An NSI request may be responded via creation of a new slice or modification of an existing one. Provisioning requests are decomposed into their constituents by corresponding management service providers. Drones can have varying roles in mobile networks, e.g., as slice consumer, slice provider, and slice constituent. . . . . . . . . . . . . . . 138 


\section{Nomenclature}

\section{List of abbreviations}

$\begin{array}{ll}\text { A-NSS } & \text { Aerial network slice subnet } \\ \text { AMF } & \text { Access and mobility management function } \\ \text { AP } & \text { Access point } \\ \text { AR } & \text { Aerial-relay } \\ \text { ATN } & \text { Aerial transport network } \\ \text { AUE } & \text { Aerial-UEs } \\ \text { BBU } & \text { Baseband unit } \\ \text { BVLoS } & \text { Beyond-visual-LoS } \\ \text { C-RAN } & \text { Cloud radio access networks } \\ \text { CAPEX } & \text { Capital expenditure } \\ \text { CDF } & \text { Cumulative distribution function } \\ \text { CR } & \text { Corner region } \\ \text { CU } & \text { Central unit } \\ \text { DAS } & \text { Distributed antenna systems } \\ \text { DC } & \text { Dual connectivity } \\ \text { DL } & \text { Downlink }\end{array}$




\begin{tabular}{ll} 
DMF & Drone-BS management framework \\
DU & Distributed unit \\
Drone-BS & Drone-base-station \\
Drone-SgNB & Drone-secondary-gNB \\
E-UTRA & Evolved universal terrestrial radio access \\
EN-DC & E-UTRA - NR DC \\
EPC & Evolved packet core \\
FCAPS & Fault, charging, authorization, performance, and security \\
FDM & Frequency-division multiplexing \\
FSO & Free-space optical communication \\
GCS & Ground control station \\
HAP & High altitude platform \\
HetNet & Heterogeneous network \\
IAB & Integrated access and backhaul \\
IGASC & Integrated ground-air-space communication \\
IMSI & International mobile subscriber identity \\
InP & Infrastructure provider \\
IoT & Internet of things \\
JSNC & Joint spatial network configuration \\
LAA & Licensed-assisted access \\
LAP & Low-altitude platforms \\
LTE & Long term evolution \\
LTE-A & Long term evolution advanced \\
LoS & Line-of-sight \\
\hline
\end{tabular}




$\begin{array}{ll}\text { MED } & \text { Mobile-enabled drone } \\ \text { MILP } & \text { Mixed integer linear program } \\ \text { MIMO } & \text { Multiple-input multiple-output } \\ \text { MINLP } & \text { Mixed integer non-linear problem } \\ \text { MIQCP } & \text { Mixed integer quadratically constrained problem } \\ \text { MME } & \text { Mobility and management entity } \\ \text { MNO } & \text { Mobile network operator } \\ \text { MR-DC } & \text { Multi-RAT DC } \\ \text { MeNB } & \text { Macro master-eNB } \\ \text { MgNB } & \text { Master-gNB } \\ \text { MmWave } & \text { Millimeter wave } \\ \text { NE-DC } & \text { NR - E-UTRA DC } \\ \text { NEF } & \text { Network exposure function } \\ \text { NOE } & \text { Notwork slice management function } \\ \text { NF } & \text { Network function } \\ \text { NFMF } & \text { Network function management function } \\ \text { NGE } & \text { Network function virtualization } \\ \text { NG } & \text { Next generation } \\ \text { NG-RAN E-UTRA-NR DC }\end{array}$




\begin{tabular}{|c|c|}
\hline NSS & Network slice subnet \\
\hline NSSI & Managed network slice-subnet instances \\
\hline NSSMF & Network subnet slice management function \\
\hline NSaaS & Network-slice-as-a-Service \\
\hline NTN & Non-terrestrial network \\
\hline NWA & PDCP-level NR and WLAN \\
\hline NWIP & IP-level NR and WLAN \\
\hline OMA & Orthogonal multiple access \\
\hline OPEX & Operational expenditure \\
\hline PDCP & Packet data convergence protocol \\
\hline PL & Path loss \\
\hline $\mathrm{PM}$ & Performance management \\
\hline PM & Probabilistic model \\
\hline QoS & Quality of service \\
\hline RAN & Radio access network \\
\hline RAT & Radio access technology \\
\hline RHS & Right-hand-side \\
\hline RL & Reinforcement learning \\
\hline RLC & Radio link control \\
\hline $\mathrm{RN}$ & Relay nodes \\
\hline RRH & Remote radio head \\
\hline $\mathrm{Rx}$ & Receiver \\
\hline S-NSSAI & Single network slice selection assistance information \\
\hline SA & System and architecture \\
\hline
\end{tabular}




\begin{tabular}{|c|c|}
\hline SBA & Service based architecture \\
\hline SCG & Secondary cell group \\
\hline SD & Slice differentiator \\
\hline SDAP & Service data adaptation protocol \\
\hline SDM & Space-division multiplexing \\
\hline SDN & Software defined networking \\
\hline SE & Spectral efficiency \\
\hline $\mathrm{SMF}$ & Session management function \\
\hline $\mathrm{SNC}$ & Spatial network configuration \\
\hline SNR & Signal-to-noise ratio \\
\hline SOS3 & Special ordered set of type 3 \\
\hline SP & Service provider \\
\hline SP & Service provider \\
\hline SST & Slice/service type \\
\hline TD & Temporal-difference \\
\hline TDM & Time-division multiplexing \\
\hline $\mathrm{TP}$ & Transmit point \\
\hline $\mathrm{TR}$ & Technical recommendation \\
\hline TS & Technical standardization \\
\hline $\mathrm{Tx}$ & Transmitter \\
\hline UAV & Unmanned aerial vehicle \\
\hline UBeAS & User-behavior-aware Stackelberg repeated game \\
\hline UCM & Urban canyon model \\
\hline UCNC & User-centric no-cell \\
\hline
\end{tabular}




$\begin{array}{ll}\text { UE } & \text { User equipment } \\ \text { UL } & \text { Uplink } \\ \text { ULAP } & \text { Ultra-low-altitude platforms } \\ \text { UPF } & \text { User-plane function } \\ \text { USNC } & \text { Uncoordinated spatial network coordination } \\ \text { VNF } & \text { Virtual network function } \\ \text { VWA } & \text { Virtual wireless access } \\ \text { WID } & \text { Wireless infrastructure drone }\end{array}$




\title{
Frequently used symbols
}

\author{
$x_{D} \quad$ Position of the drone-BS in x-dimension \\ $y_{D} \quad$ Position of the drone-BS in y-dimension \\ $h, h_{D} \quad$ Altitude of the drone-BS \\ $h_{R} \quad$ Height of receiver \\ $h_{B} \quad$ Height of human blockers \\ $g_{B} \quad$ Diameter of a human blocker \\ $r_{i} \quad$ Horizontal distance between the drone-BS and $i^{\text {th }}$ user \\ $e_{i} \quad 3 \mathrm{D}$ distance between drone-BS and $i^{\text {th }}$ user \\ $f_{c} \quad$ Carrier frequency $(\mathrm{Hz})$ \\ c Speed of light $(\mathrm{m} / \mathrm{s})$ \\ $\eta_{\text {LoS }} \quad$ Loss of the LoS connection (dB) \\ $\eta_{N L S S} \quad$ Loss of the NLoS connection (dB) \\ $\gamma \quad$ Pathloss corresponding to the QoS requirement \\ $L\left(h, r_{i}\right) \quad$ Pathloss of a user that is $r_{i}$ away from a drone-BS at altitude $h$ \\ $u_{i} \quad$ Binary variable indicating whether user $i$ is served by the drone-BS, or not \\ U Set of users that are not covered by the macrocell \\ $R \quad$ Radius of the area to be covered \\ $\alpha \quad$ Ratio of altitude to the radius of the coverage area \\ $N_{u} \quad$ Number of users \\ $\tau_{i} \quad$ Incentive offered to user $i$ \\ $d_{i} \quad$ Spatial displacement amount offered to user $i$ \\ $\beta\left(\tau_{i}\right) \quad$ Persuasion parameter
}

$\Gamma(\alpha) \quad$ Maximum allowable distance for coverage by a drone-BS as a function of $\alpha$ 


$\begin{array}{ll}\Gamma^{*} & \text { Maxima of } \Gamma(\alpha) \\ \Pi\left(\tau_{i}, d_{i}\right) & \text { Average unit profit from a user } d_{i} \text { meters away from the coverage region with incentive } \tau_{i} \\ \mathcal{R} & \text { Coverage area of the drone-BS } \\ W & \text { Area where users are distributed } \\ \mathcal{D} & \text { Extended coverage area where incentives are offered (UIL area) } \\ \mathrm{P}^{\mathrm{R}} & \text { Probability that a user is covered } \\ \mathrm{P}^{\mathrm{D}} & \text { Probability that a user is covered in the UIL area } \\ \mathrm{P}^{\mathrm{W}} & \text { Coverage probability of a user in } \mathcal{W} \\ \Pi_{\circ}(\tau) & \text { Average normalized revenue with regional incentive } \\ \Pi_{N}(\tau) & \text { Average normalized revenue in a region with } N \text { users } \\ \Pi_{N}^{\infty}(\tau) & \text { Limit of } \Pi_{N}(\tau) \text { for an infinite region } \\ \lambda & \text { User density }\end{array}$




\section{Chapter 1}

\section{Introduction}

From tactile Internet to Internet of things, the future of wireless services is as exciting as it is challenging. The main challenge is that the ever-increasing wireless demand is expected to grow in different dimensions due to varying requirements of new applications. Very low latency, very high data rate, or very high energy efficiency can be listed among those requirements. As a straightforward remedy to these challenges, densification of wireless networks seems to be inevitable [1]. On the other hand, leaning to gross over-engineering comes at a high cost of not only CAPEX and OPEX (capital and operational expenses, respectively), but also environmental footprint. Moreover, applications have requirements with demands at opposing extremes, which makes it hard to design robust networks that can respond to all requirements efficiently. Therefore, flexible and agile networking solutions gained importance. Technologies enabling on-demand network configuration and programming, such as network function virtualization (NFV) and software defined networking (SDN), became integral parts of such solutions. However, these technologies and solutions do not stem from wireless networks; they are from the field of wired networking. Therefore, they do not consider all degrees of freedom that wireless networks can utilize. Note that wireless networks are fundamentally designed to support user mobility. This thesis investigates whether that capability can be used to support mobility of the access points, and eventually, help to create a completely flexible and customizable wireless net-

work. From a wide perspective, we try to understand whether support for "mobility" can be turned into network "agility". In particular, we investigate characteristics of utilizing unmanned aerial vehicles (UAVs) at medium to very low altitudes, also-known-as drones as moving access points, i.e., drone-base-stations (drone-BSs) [2]. 
Using aerial devices as moving access points recently gained significant attention due to their advantages such as agility and manoeuvrability [3-7]. Accordingly, the literature on drone-BSs boomed with numerous studies from various aspects. One of the most fundamental aspects is air-to-ground channel modelling, since it is necessary to have accurate models for efficient utilization of aerial devices. However, the studies on air-to-ground channel modelling are not as comprehensive as the ones for terrestrial channel models yet. Thorough surveys are provided in [8] and [9] on available air-to-ground channel measurement campaigns, large and small scale fading channel models, air-to-ground, groundto-ground, air-to-air channel measurements and modeling, and future research directions. One of the reasons slowing down the air-to-ground channel modeling is the need to conduct extensive measurement campaigns in different environments. As exemplary and recent attempts, studies in [10-12] provide measurement-based analysis for air-to-ground channel models in over-water, hilly and mountainous, and suburban and near-urban environment settings. Another challenge is to account for effects of machinery [13] and channel types, such as multiple-input multiple-output (MIMO) [14-21]. The study in [13] presents the results of shadowing depth, duration, multiple antenna diversity gain, and small scale fading results and their statistics based on over 200 aircraft wing/engine shadowing events in both C-band (5060 MHz) and L-band (968 MHz). Authors also provide algorithms to simulate shadowing events and show that multiple antennas help mitigate negative effects. 3D geometry-based air-to-ground channel models are studied in [14-17]. The study in [14] proposes a practical model based on numerical analysis by considering ground surface and roadside environment reflections particularly for vehicle-to-vehicle communications. Alternatively, the study in [15] performs measurements, and proposes a model considering line-of-sight (LoS), ground reflection, and truncated ellipsoid scattering components. In addition to the components [15], a cylinder model, and multiple confocal truncated ellipsoid models are considered in [16], so that the proposed geometry-based stochastic model has the ability to incorporate the impact of drone heights and transceivers' movements on channel characteristics in diverse environments. Besides developing deterministic and stochastic simulation models based on the reference model, [17] also proposes a wideband model using a single concentric-cylinders model, where the signal is a superposition of a line-of-sight component and single-bounced rays. In order to describe the non-stationarity of the channel, time-varying angles have been properly derived and added in the proposed model. Since drones will be a part of current and future wireless networks, both as user equipments and aerial access nodes, the studies in [22] and [23] provide valuable channel 
sounding experiment results and insights on air-to-ground communications based on Long Term Evolution (LTE) signals. Furthermore, urban environments are particularly interesting for a variety of applications of airborne communications, such as disaster recovery, and increasing capacity of wireless networks for temporary events. Although regulatory limitations and safety concerns decelerate conducting comprehensive measurement campaigns in urban setting, studies in [24-30] exemplify attempts in investigating air-to-ground channels in urban environments with alternative approaches: While [24] proposes a learning-based method to predict air-to-ground channel quality along the trajectory of a drone-BS, [29] proposes to utilize urban maps for more accurate path loss predictions. Both studies alter existing channel models and [29] relies on ray tracing simulations to validate results. Statistical modeling methods are utilized in [25-28] to obtain path loss expressions. Among such models, the ones presented in [27] and [28] are widely used in the literature since they model the relationship between altitude and path loss of a drone-BS in a tractable way. According to the models in [27] and [28], it is possible to derive an optimal altitude for a fixed transmission power, where up to that altitude the coverage area of a drone-BS increases due to increasing probability of establishing LoS air-to-ground links. However, altitudes higher than the optimal altitude result in a smaller coverage area due to increased air-to-ground distance. In fact, this characteristic of air-to-ground links triggered the $3 D$ placement of drone-BS, which is one of the approaches to the aerial node positioning problem.

The location of aerial nodes may not be known before-hand, and it is necessary to determine the best positions for efficient operation of aerial nodes. Therefore, aerial node positioning rapidly became a very active research field, as it is related to one of the most significant characteristics, the mobility, of drone-BSs [3-5,31-47]. Operation type and aim can be one of the determining factors for the best position. In [31-36,47] covering maximum number of users is one of the main criteria to determine optimal position(s) of one or more drone-BSs in a variety of scenarios including boosting insufficient capacity of wireless networks, recovery after disasters, and supporting wireless networks for temporary events. The studies in [31] and [33] have similar convex optimization based approaches to maximize the number of covered users. However, the study in [33] extends [31] for users with different QoS requirements. Differently, heuristic and greedy algorithms, such as particle swarm optimization, are used in [34-36,48-50] to reduce the computational difficulties of the previous studies with as minimal compromise from the optimal solution as possible. Since a variety of scenarios require multiple drone-BSs to be utilized, e.g., 
to continuously serve the users scattered in a large area, [34-36, 40, 42, 43, 48-65] investigate positioning of multiple drone-BSs. One of the distinctive properties of these studies is whether 3D or 2D placement is considered. While studies such as $[34,35,40,42,48-50]$ consider placement in 3D, studies in [43,54-56] assume a fixed altitude. Trajectory optimization, i.e., where drone-BS follows a trajectory instead of hovering at a single optimal location to serve users, is considered among some studies on 2D placement, such as [54] and [43]. For instance, in [43] a drone-BS is responsible to multicast a common file to a set of users on the ground. The trajectory of the drone-BS is optimized to minimize its mission completion time, while ensuring that users obtain the file with a certain probability. Similarly in [54], multiuser communication scheduling and UAV trajectory over a finite horizon is jointly optimized by an iterative algorithm and the proposed low-complexity initialization scheme. To further extend the positioning problems, frameworks that jointly solve positioning and related issues are developed in the literature from various aspects. For instance, [47] combines user involvement and 3D placement. In [65], the 3D placement is performed by considering fairness metric with the particle swarm optimization algorithm. There are a number of studies on joint spectrum management (backhaul and access resource allocation) and positioning [35,66,67]. The study in [66] develops a joint backhaul resource allocation (by terrestrial BSs), and terrestrial BS and drone-BS access selection framework to support IoT networks with multiple drone-BSs. This complex problem is solved by using a game theoretical framework, whereas heuristic methods are preferred in [35]. In addition to the limited resources of spectrum, energy of drone-BSs is another concern. Therefore, jointly managing energy efficiency and positioning of the drone-BSs has attracted significant attention [32,48, 52, 68-70]. In [68] optimal 3D positions of drone-BSs are obtained by considering travelling and communication energy consumption of drone-BSs with cognitive radio technology. In [69], the lifetime of a drone-BS based communication network is defined based on the recall frequency of drones, i.e., when they need to be recalled to be recharged. Then, the optimal placement minimizing the recall- frequency and consumption of on-board circuit power consumption is investigated by considering environmental effects, such as dense scattering environments, and user density. Although [52] mainly focuses on minimizing energy consumption of IoT devices served by drone-BSs, the results also provide energy efficient paths for drone-BSs to serve the underlying network. The study in [70] provides energy efficiency by adjusting transmit power, and shows the existence of an optimal altitude when the relative position of drone-BS is considered with respect to surrounding access points. The drone-BS placement problem is decoupled in [32] 
based on the vertical and horizontal dimensions without loss of optimality, and the problem in horizontal dimension is formulated as a circle placement problem and a smallest enclosing circle problem. Hence, the optimal placement is obtained to maximize the number of covered users using the minimum transmit power. Since most of the performance criteria depend on user-BS association, e.g., throughput, joint placement and user association is investigated either explicitly or inherently by many studies [40, 50, 53, 71-73].

There are many other notable and interesting fields in the recent literature of droneBSs with ample room to contribute. Caching is investigated in [57] and [74]. A couple of drone-BS management frameworks (DMFs) are proposed in the literature. DMF can have different focuses: The studies in [2] and [75] consider various business models and focus on ensuring a profitable operation. The framework proposed in [76] aims at improving spectrum efficiency by data and control plane separation for drone-BS and drones equipped with IoT devices. In fact, supporting IoT networks is another rich study field for drone-BSs, with respect to their positioning, resource allocation, management, security and energy efficiency [53,66,76-86]. A joint 3D placement, drone-BS trajectory optimization and user association framework is proposed in [53] by considering energy limitations of both drones and IoT devices. A similar problem with drone-BS deployment for IoT networks is solved via low-complexity heuristics in [79]. In addition, resource allocation is considered in [66]. Data collection from IoT devices and wireless power transfer to them via drone-BSs is investigated in [77] and [86]. Wireless powered communication networks are investigated in [82] for throughput maximization where drone-BSs both collect data from IoT devices and transfer energy to them via RF links. The study in [81] proposes an efficient solution for a MIMO-based data collection scheme with drone-BS trajectory optimization and partial channel state information. Authors propose to utilize compressive data gathering in [85], such that the drone-BSs prevent multihop transfers in power-critical wireless sensor networks. The study in [78] provides a survey on interactions between drones and IoT devices from the perspective of smart cities, including data collection, privacy and security, public safety, disaster management, energy consumption and quality of life in smart cities. In [80], a general analytical framework based on stochastic geometry is proposed to compute the uplink and downlink coverage probabilities for both the aerial and the terrestrial cellular system over IoT networks. Analytical results are supported by Monte Carlo simulations, and the authors show the impact of environment and drone-BS altitude on network performance. Joint drone-BS hovering altitude selection and power allocation is performed via a two-stage algorithm in [83] to ensure efficient communications. 
Not only IoT devices, but also drone-BSs themselves have limited resource. Due to limited resources of drone-BSs and potential need to support massive communications, non-orthogonal multiple access (NOMA) is investigated from various aspects for droneassisted wireless networks: In [75] a framework for heterogenous aerial and ground wireless networks is proposed to maximize profitability by considering NOMA and orthogonal frequency division multiplexing (OFDM) constraints. In [87], it is shown with simulations that a mmWave-drone-BS can achieve higher spectral efficiency with beamforming and NOMA compared to orthogonal multiple access (OMA), and there is an optimal transmit power value with respect to the improvement in outage sum rate. Similarly, outage behaviour is used as a performance criteria in [88], where a drone-BS is used as a decodeand-forward relay. Results show that the QoS performance of the edge user requires an adequate altitude for drone-BSs, and the performance is superior to OMA. In [89], effects of limited feedback on mmWave-drone-BSs is compared for NOMA and OMA. It is shown that mmWave-drone-BSs have better sum rates compared to OMA, when there is limited feedback from users regarding either users' distance, or users' angle with respect to the beamforming direction under different user region geometries. Sum-rate, coverage, and energy efficiency of NOMA-based drone-BSs are investigated in [90], where fixed-altitude NOMA schemes are compared with altitude-optimized NOMA schemes. A NOMA-based multi-beam transmission strategy is proposed in [91]. The placement and power allocation are jointly optimized to improve the performance of the NOMA-based drone-BSs in [92]. In [93], dominant factors for outage performance is investigated for NOMA-based droneBSs. It is shown that power allocation factor is effective for users at the edge, whereas ground users in proximity are strongly affected by the channel and trajectory parameters. A NOMA-based cooperative transmission scheme is proposed for drone-BS aided wireless backhaul transmissions in [94]. Drone-BSs and terrestrial BSs cooperate to serve ground users in [95], where the trajectory of the drone-BSs are optimized to maximize sum rate, and NOMA precoding vectors are optimized to minimize interference between terrestrial BSs and drone-BSs. In [96], in order to mitigate eavesdropping attacks, obtaining a sizeoptimized protected region around users is proposed by considering limited resources of drone-BSs. It is shown that the protected region size needs to be optimized for each altitude of drone-BSs.

In fact, security of drone-BSs communications is important for their command\&control links as well as uplink, downlink and wireless backhaul. Providing sufficient security with limited resources of a drone-BS and challenging air-to-ground links is a cumbersome 
problem that is lightly investigated so far [5, 97, 98]. Furthermore, mmWave links and device-to-device (D2D) communications are considered in several studies for drone-BSs as in [87-89,96,99] and in [84,100-104], respectively. Mobility of drone-BSs change the dynamics of network planning, which is another lightly investigated issue with complex dimensions such as, the distribution of the users, properties of the underlying terrestrial network, and demand distribution in space and time [39,42, 105-107].

As discussed so far, there are many aspects to consider for drone-BSs and wireless networks. Hence, this study is multi-faceted: First, we propose an efficient 3D placement scheme and investigate its application for mmWave-based drone-BSs as well. Then, we propose the Spatial Network Configuration (SNC) method, where agility and flexibility are provided to wireless networks via exploiting the mobility of access points and users. Unmanned aerial vehicles, also-known-as drones, equipped with functionalities of terrestrial base stations [2] serve as moving access points in the SNC framework. On the other hand, the mobility of the users are exploited via user-in-the-loop, a method to offer incentives to users, that is specifically developed for wireless networks. Another aspect of this study is the channel modelling and we propose a map-based channel model with superior accuracy compared to a widely used channel model. Finally, we propose novel network management and planning frameworks, and investigate the latest developments in $5 \mathrm{G}$ standardization framework thoroughly.

The rest of this dissertation is organized as follows. In Chapter 2, we introduce the 3D placement problem of a drone-BS. First, we propose an efficient solution method for droneBSs operating at sub-6GHz frequencies. Then, we develop a similar method for mmWavedrone-BS by utilizing a channel model which considers the human body blockage ratio.

We introduce the spatial network configuration framework in Chapter 3. The SNC framework takes both the movement of the aerial access point and users into consideration. We propose three SNC schemes and solution methods. Our results show that SNC is a promising method to increase profitability and capacity of the networks simultaneously in congested scenarios.

In Chapter 2.3, we contribute to air-to-ground channel modeling for drone-BSs utilized in metropolitan areas. The number of studies on air-to-ground channel models are quite less compared to the studies on terrestrial channel models. Despite the global urbanization trend, the studies on air-to-ground channels in environments with high rise buildings are 
even more deserted. Our study is one of the most recent and unique studies in this area that suggests using the information about the environment, e.g., height of the buildings and width of the streets, to estimate average path loss more accurately. We validate the proposed channel model with ray tracing simulations, and show that the proposed model is more accurate compared to a widely used air-to-ground channel model. Furthermore, we demonstrate that increasing accuracy of channel model has critical implications for droneBS operations, such as planning for the required number of drone-BSs to cover outdoor urban users.

Chapter 4 has a system-level approach, where we first investigate the roles of drones in next generation multi-tier networks, enabling technologies and challenges. Then, we propose the first DMF for wireless networks in the literature. We present a use case where DMF utilizes 3D placement method in multi-tenant networks via enabling technologies such as NFV, SDN, and big data analysis. We further investigate the integration of drone$B S s$ into wireless networks by considering virtual wireless access, and show that the impact of the integration method on the performance gain. In order to evaluate the practicality of the integration methods, and understand the situation of the current and prospective wireless networks, we investigate the 5G Phase-I standardization. Our evaluation indicates that future networks can be capable of integrating drones efficiently, yet, collaborations between the industry and academia is necessary to develop comprehensive solutions. Finally, we summarize our studies and observations in Chapter 6 .

\subsection{List of Publications}

Following is a list of publications where the contributions of this thesis appeared.

\subsubsection{Published Journal Papers — included in the thesis}

[J1] Irem Bor-Yaliniz, Mohamed Salem, Gamini Senerath, and Halim Yanikomeroglu, "Is 5G ready for drones: A look into contemporary and prospective wireless networks from a standardization perspective", IEEE Wireless Communications Magazine, Special Issue on Integrating UAVs into 5G and Beyond, vol. 26, no. 1, pp. 18-27, February 2019. 
[J2] Irem Bor-Yaliniz, Amr El-Keyi, and Halim Yanikomeroglu, "Spatial configuration of agile wireless networks with drone-BSs and user-in-the-loop", in IEEE Transactions on Wireless Communications, vol. 18, no. 2, pp. 753-768, February 2019.

[J3] Irem Bor-Yaliniz, Sebastian S. Szyszkowicz, and Halim Yanikomeroglu, "Environment aware drone-base-station placements in modern metropolitans", IEEE Wireless Communications Letters, vol. 7, no. 3, pp. 372-375, June 2018.

[J4] Irem Bor-Yaliniz and Halim Yanikomeroglu, "The new frontier in RAN heterogeneity: Multi-tier drone-cells", IEEE Communications Magazine, vol. 54, no. 11, pp. 48-55, November 2016.

\subsubsection{Published Journal Papers - not included in the thesis}

[J5] Faraj Lagum, Irem Bor-Yaliniz, and Halim Yanikomeroglu, "Strategic densification with UAV-BSs in cellular networks", IEEE Wireless Communications Letters, vol. 7 , no. 3, pp. 384-387, June 2018.

\subsubsection{Submitted Journal Papers - not included in the thesis}

[J6] Jalal Khamse-Ashari, Gamini Senarath, Irem Bor-Yaliniz, Halim Yanikomeroglu, "An agile and distributed mechanism for network slicing in next-generation mobile networks", IEEE Transactions on Mobile Computing, November 2019.

\subsubsection{Published Conference Papers - included in the thesis}

[C1] Irem Bor-Yaliniz, Gamini Senarath, and Halim Yanikomeroglu, "Aerial Access Nodes and Virtual Wireless Access: A Look into Integration Strategies" to appear in IEEE International Conference on Communications (ICC), 7-11 June 2020.

[C2] Margarita Gapeyenko, Irem Bor-Yaliniz, Sergey Andreev, Halim, Yanikomeroglu, and Yevgeni Koucheryavy, "Effects of blockage in deploying mmWave drone base stations for beyond-5G networks", Invited Paper in IEEE International Conference on Communications Workshops (ICCW), 20-24 May 2018, Kansas City, MO, USA. 
[C3] Irem Bor-Yaliniz, Amr El-Keyi, and Halim Yanikomeroglu, "Efficient 3-D placement of an aerial base station in next generation cellular networks", in IEEE International Conference on Communications (ICC), 23-27 May 2016, Kuala Lumpur, Malaysia.

\subsubsection{Published Conference Papers - not included in the thesis}

[C4] Elham Kalantari, Irem Bor-Yaliniz, Abbas Yongacoglu, and Halim Yanikomeroglu, "User association and bandwidth allocation for terrestrial and aerial base stations with backhaul considerations", Invited Paper in IEEE International Symposium on Personal, Indoor, and Mobile Radio Communications (PIMRC), 08-13 October 2017, Montreal, Quebec, Canada.

\subsubsection{Patent Filings - not included in the thesis}

[P1] “Multi-level intent driven network management", requested [16 February 2019].

[P2] “Network slice provisioning and operation", US. Pat. App. No. 16/243, published [11 January 2019].

[P3] "Method and apparatus for the specification of a network slice instance and underlying information model," U.S. Pat. App. No. 16/186,266, filed [15 November 2018].

[P4] "Method and system of performance assurance with conflict management in provisioning a network slice service”, U.S. Pat. App. No. 16/670,962, filed [09 November 2018].

[P5] "Management of network slices and associated services", US Patent App. No. 16/146,345, filed [28 September 2018].

[P6] "Methods and functions of network performance monitoring and service assurance", U.S. Pat. App. No. 16/526,170, filed [31 August 2018].

[P7] "Systems and method for managing capacity and coverage in communication networks", U.S. Pat. App. No. 16/541,305, filed [17 August 2018]. 
[P8] "Data analytics management (DAM), configuration specification and procedures, provisioning, and service based architecture (SBA)", U.S. App. No. 16/441,940, filed [17 August 2018].

[P9] "Communication service and network slice lifecycle management," U.S. Pat. Ser. No. PCT/CN2018/100121, filed [11 August 2018].

[P10] "Method for performance assurance with intent-driven networking", U.S. Provisional Pat. Ser. No. 62/758,354, filed [3 August 2018].

[P11] "Method of communication service management and assurance", U.S. Provisional Pat. Ser. No. 62/714,438, filed [3 August 2018].

[P12] "System and method for network function, network slice subnet and network slice resource usage calculation and prediction", U.S. Provisional Pat. Ser. No. 62/688,603, filed [22 June 2018].

[P13] "Service based architecture (SBA) for data analytics management (DAM), configuration specification and procedures”, U.S. Provisional Pat. Ser. No. 62/688,603, filed [11 June 2018].

[P14] "Lifecycle management of NSI and CSI", U.S. Patent App. No. 16/058,965, filed [11 August 2017]. 


\section{Chapter 2}

\section{3-D Drone-BS Placement}

Benefiting from agility of low-altitude unmanned aerial vehicles (UAVs) can bring us to the next frontier of wireless heterogeneity [2]. They can provide on-demand capacity as rapidly deployable base stations, i.e., drone-base-stations (drone-BS). However, on-demand utilization of drone-BSs must be as efficient as possible due to high operational cost of droneBS. For instance, the current technology of drones/unmanned aerial vehicles (UAVs) do not allow them to be used in a fully automated fashion, i.e., at least one remote pilot needs to be flying the drone. Furthermore, although technically one drone-BS may be sufficient for providing the desired capacity, there may be a need to utilize several drone-BSs to enhance reliability. In addition, the energy cost of drone-BSs can be a critical factor due to the limited battery life.

The efficiency of drone-BS operations can be obtained by considering characteristics of drone-BS communications. Some characteristics, such as weather conditions and aircraft choice are rather machinery-related and environmental conditions. Those characteristics can be considered out-of-scope from the perspective of communication systems engineering. Accordingly, throughout this thesis, a drone-BS is assumed to be static once positioned. Hovering stability of UAVs is an active research field, as well as a competitive development area for commercial drone producers [108-111]. Since the machinery of drones are designed based on the mission, unavailability of a drone-BS makes it impossible to comment on stability of a drone-BS with certainty. Drone types and design issues are discussed in more detail in Section 4.1.2. From our perspective, two fundamental characteristics are prominent in managing efficiency of drone-BSs: Air-to-ground communication 
links and drone-BS positioning. These factors help determine the most efficient baselines for meeting the communication demand. Moreover, snapshot analysis is conducted which implies that the user mobility is not considered. That assumption is conformable with the assumptions on stability of the hovering drone, as well as the air-to-ground channel model that is based on averages on environment. Certainly, this model would not be useful if the computation time to place the drone-BS is relatively long compared to the user mobility. On the other hand, the 3-D placement method presented in this thesis alleviates many common assumptions for the first time in the literature as discussed in Section 2.1.

There is an intertwined relationship between the positioning of a drone-BS and air-toground channels quality. Air-to-ground channels differ from terrestrial channels with their higher likelihood of having line-of-sight (LoS). The likelihood of LoS depends on two factors:

- Type of environment, e.g., density and average height of the buildings, width of the streets.

- 3-D distance between the user and the drone-BS, which determines the elevation angle.

In most of the studies in the literature either the altitude or the horizontal location of drone-BSs are assumed to be pre-determined, which makes the problem very similar to small-cell placement problems, and extenuates the relationship between the 3-D position and air-to-ground link quality. In contrast, our approach is based on the 3-D placement of a drone-BS, i.e., jointly determining altitude and horizontal location of a drone-BS with respect to the quality of the air-to-ground links. This problem is introduced and efficiently solved in [31]. Then, a multi-objective 3-D placement problem considering multi-tenancy, energy efficiency, caching and congestion release is formulated in [2], and caching is investigated further in [57]. Multi-tier drone-BS placement is investigated to show potential gains in spectral efficiency, throughput, latency and coverage in [112], and a drone-BS network formation algorithm is developed in [113] by considering 3-D placement. Spectrum sharing between single-tier and multi-tier drone-BSs is investigated to determine the optimal density of drone-BSs in [114] by assuming pre-determined horizontal locations for drone-BSs. Terrestrial users are clustered to determine placement of drone-BSs in the horizontal domain in [35]. In [55], assuming a fixed coverage area for drone-BSs, a 
polynomial-time algorithm is developed to provide maximum coverage to a finite area with minimum number of drone-BSs. In [61], optimum hovering positions of drone-BSs with antenna arrays are determined to minimize interference and maximize signal-to-noise ratio (SNR). The proposed method is validated by exhaustive search, and provides computational efficiency and higher capacity performance by combining linear zero-force beamforming with transmit beamforming. Improving resilience of wireless networks is investigated in [46,115-117]. Downlink coverage analysis is conducted for a single drone-BS in [116], and for a 3-D drone-BS network in [118]. Although studies discussed so far consider hovering drone-BSs, an energy efficient trajectory is determined for point-to-point communications between a fixed-altitude drone-BS and terrestrial users in [119], and for drone-BSs with a fixed coverage area and altitude in [52]. Other studies worth noting investigate the issues of releasing congestion, power allocation for drone-BSs, association problem in cloud radio access network (C-RAN) with drone-BSs, network performance analysis, drone-BSs as moving edge infrastructures, and development of placement and trajectory optimization algorithms from various aspects [32, 43, 51, 54, 58, 60, 120-125]. Finally, in [99], effects of mobile access points on business models are discussed.

\subsection{Efficient 3-D Placement}

As discussed in the prior section, there are three main approaches to the drone-BS placement:

- Assuming a simplified channel model and/or one of the dimensions of the drone-BS location is pre-determined,

- Assuming a simplified channel model and maintaining LoS by determining a trajectory for the drone-BS,

- Utilizing a channel model that incorporates air-to-ground channel and environment dynamics.

This study introduced the first efficient solution for the last approach above, i.e., 3-D placement. In this framework, placement of a drone-BS is different than terrestrial cell placement because of the following reasons: 


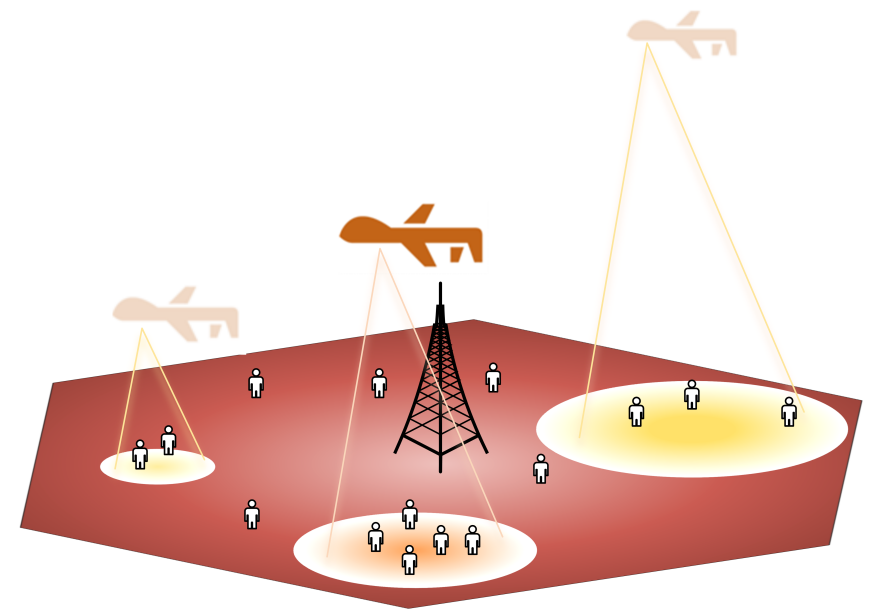

Figure 2.1: A possible scenario showing the users that are not covered by the eNB. Three potential placements of a drone-BS is highlighted.

1. In addition to choosing the location of the drone-BS in the horizontal space $\left(x_{D}, y_{D}\right)$, we need to determine its altitude, $h$, as well.

2. The coverage area of a terrestrial cell is known a priori. However, the coverage area of a drone-BS depends on its altitude, and is unknown before solving the placement problem.

3. The mobility of the drone-BS allows it to move wherever the demand is, rather than terrestrial cells waiting for the demand to come towards them. As a result, the coverage region providing the maximum revenue to the network should be found.

The first item indicates that the placement of the drone-BS is a 3-D problem. In addition, the last two items, which are determining the size of the coverage area and identifying the location of the coverage area, must be considered jointly.

A possible placement problem is shown in Fig. 2.1. Assume that a macrocell is congested, where only the users that cannot be served by the eNB are shown. Three potential areas to be covered by deploying a drone-BS at different altitudes and locations are highlighted. Note that in each case, as well as the altitude of the drone-BS, the size of the area to be covered is changing, which in turn, affects the number of users served by the drone-BS. In this section, we will formulate and solve the 3-D placement problem efficiently to serve the maximum number of users with the minimum required 
area. Due to the complexity of the air-to-ground channel model, the solution of the 3-D placement problem formulation cannot be directly found. In order to solve this problem, we introduce a new variable relating the altitude of the drone-BS to the radius of its coverage area. Although there is not an analytical expression for the optimal value of this variable, it can be efficiently obtained by using one dimensional bisection search. Afterwards, the 3-D placement problem reduces to a mixed-integer non-linear problem (MINLP), which can be solved by using the interior point optimizer of the MOSEK solver.

\subsubsection{System Model}

Consider a macrocell where the location of each user $i$ is known and represented by $\left(x_{i}, y_{i}\right)$. We assume that for a user to be served, the QoS measured by the received signal-to-noise ratio (SNR) should be above a certain threshold. In case of an extreme event, such as congestion within the cell, or malfunction of the infrastructure, the terrestrial base station may become unable to serve all users. Hence, it will be assisted by a drone-BS with fixed transmission power. We consider a low-altitude quasi-stationary UAV for this purpose, and would like to determine the altitude $h$, and location, $\left(x_{D}, y_{D}\right)$, providing the maximum revenue. Assuming a fixed QoS for all users, the maximum revenue can be obtained by offloading the maximum amount of users to the drone-BS. The placement of the drone-BS affects both the number of users enclosed by its coverage region, and the quality of the airto-ground links. Utilization of air-to-ground links is a characteristic of aerial base stations. There has been several studies on air-to-ground channel models, which we discuss next.

The air-to-ground channel differs from the terrestrial channel due to its higher chance of LoS connectivity. As a result, Rician [126], large scale Rayleigh [127], and free space fading [128] models are widely utilized in the literature for air-to-ground channels. However, none of them considers the effect of the environment on the occurrence of LoS. One of the most complete models on the effects of building blockage on radio propagation is proposed by ITU in [129]. With the help of the results in [129], a channel model for air-to-ground communication in urban environments is presented in [28] and [27], and adopted here.

The probability of having LoS for user $i$ depends on the altitude of the drone$\mathrm{BS}, h$, and the horizontal distance between the drone-BS and $i^{\text {th }}$ user, which is $r_{i}=$ 
$\sqrt{\left(x_{D}-x_{i}\right)^{2}+\left(y_{D}-y_{i}\right)^{2}}$ for the $i^{t h}$ user located at $\left(x_{i}, y_{i}\right)$ and the drone-BS at $\left(x_{D}, y_{D}\right)$. The LoS probability is given by [28]

$$
P\left(h, r_{i}\right)=\frac{1}{1+a \exp \left(-b\left(\arctan \left(\frac{h}{r_{i}}\right)-a\right)\right)},
$$

where $a$ and $b$ are constant values that depend on the environment. In this setting, the altitude of the user, and the antenna heights of both the users and the drone-BS are neglected. Then the path loss expression becomes [28]

$$
L\left(h, r_{i}\right)=20 \log \left(\frac{4 \pi f_{c}}{c}\right)+20 \log \left(\sqrt{h^{2}+r_{i}^{2}}\right)+P\left(h, r_{i}\right) \eta_{\mathrm{LoS}}+\left(1-P\left(h, r_{i}\right)\right) \eta_{\mathrm{NLoS}}
$$

where $f_{c}$ is the carrier frequency $(\mathrm{Hz}), c$ is the speed of light $(\mathrm{m} / \mathrm{s}), \eta_{L o S}$ and $\eta_{N L o S}($ in $\mathrm{dB}$ ) are respectively the losses corresponding to the LoS and non-LoS connections depending on the environment. Equivalently, (2.2) can be written as

$$
L\left(h, r_{i}\right)=20 \log \left(\sqrt{h^{2}+r_{i}^{2}}\right)+A P\left(h, r_{i}\right)+B
$$

where $A$ and $B$ are constants such that $A=\eta_{\mathrm{LoS}}-\eta_{\mathrm{NLoS}}$, and $B=20 \log \left(\frac{4 \pi f_{c}}{c}\right)+\eta_{\mathrm{NLoS}}$. Note that the path loss model presented here is a function of both $h$ and $r_{i}$. In other words, the path loss of the air-to-ground link depends on the altitude in the vertical dimension, and the distance in the horizontal dimension. Thus, we have a 3-D placement problem.

\subsubsection{Efficient 3-D Placement of a Drone-BS}

We assume that a user is in the coverage region of the drone-BS if the air-to-ground link satisfies its QoS requirement. For a given transmission power of the drone-BS, let $\gamma$ represent the pathloss corresponding to the QoS requirement. Hence, user $i$ is served by the drone-BS, if $L\left(h, r_{i}\right) \leq \gamma$. Using (2.3), we can re-write this condition as

$$
h^{2}+r_{i}^{2} \leq 10^{\frac{\gamma-\left(A P\left(h, r_{i}\right)+B\right)}{10}} \text {. }
$$

Let $u_{i} \in\{0,1\}$ denote a binary variable that indicates whether user $i$ is served by the drone$\mathrm{BS}$, or not. Using the variable $u_{i}$, which is equal to 1 , only if the user $i$ is served by the 
drone-BS, and equal to 0 otherwise, the following constraint,

$$
u_{i}\left(h^{2}+r_{i}^{2}\right) \leq 10^{\frac{\gamma-\left(A P\left(h, r_{i}\right)+B\right)}{10}}
$$

determines whether user $i$ is covered, or not. This constraint can be further manipulated to

$$
h^{2}+r_{i}^{2} \leq 10^{\frac{\gamma-\left(A P\left(h, r_{i}\right)+B\right)}{10}}+M_{1}\left(1-u_{i}\right)
$$

where $M_{1}$ is a constant that is sufficiently larger than the maximum possible value of the distance between a user and the drone-BS. Observe that when $u_{i}=1,(2.6)$ is equivalent to (2.4). If $u_{i}=0$, since $M_{1}$ is large enough, this constraint is relaxed. Now, we can continue by determining the objective function.

Assuming a fixed QoS for all users, the best region to be served by the drone-BS is identified with the maximum number of users covered. By using (2.6), the placement problem for a set of users, $\mathbb{U}$ that are not covered by the macrocell can be written as

$$
\begin{aligned}
& \underset{x_{D}, y_{D}, h,\left\{u_{i}\right\}}{\operatorname{maximize}} \quad \sum_{i \in \mathbb{U}} u_{i} \\
& \text { subject to } \\
& h^{2}+r_{i}^{2} \leq 10^{\frac{\gamma-\left(A P\left(h, r_{i}\right)+B\right)}{10}}+M_{1}\left(1-u_{i}\right), \forall i=1, \ldots,|\mathbb{U}|, \\
& x_{l} \leq x_{D} \leq x_{u}, \\
& y_{l} \leq y_{D} \leq y_{u}, \\
& h_{l} \leq h \leq h_{u} \text {, } \\
& u_{i} \in\{0,1\}, \quad \forall i=1, \ldots,|\mathbb{U}|,
\end{aligned}
$$

where $|\cdot|$ represents the cardinality of a set, subscripts $(\cdot)_{l}$ and $(\cdot)_{u}$ denote respectively the minimum and maximum allowed values for $x_{D}, y_{D}$, and $h$ of the drone-BS. Note that there are quadratic, exponential and binary terms in this problem, which makes it an MINLP. On the other hand, the fixed QoS requirement assumption simplifies the already complex problem formulation by turning $\gamma_{i}$ s into a single constant value. Although this assumption seems limiting, it may not be as impractical as it seems: First, note that $\gamma$ indicates the tolerable path loss. The tolerance level can be fixed by varying the transmit power, which is also assumed to be fixed in this study, with respect to $\gamma$. In other words, increasing the transmit power for less tolerable applications/users, and decreasing the transmit power 
with more tolerable applications/users results in a similar problem formulation. Hence, the assumptions here can be practical, if the entirety of the system is considered.We will show that this problem can be solved efficiently by using a combination of the interior-point optimizer of MOSEK solver and bisection search.

Observe that if $P(h, r)$ was a constant, then this optimization problem would be quadratically constrained MINLP. Let us denote the radius of the area to be covered by $R$ and introduce the variable $\alpha$ as

$$
\alpha=\frac{h}{R} .
$$

Then, if user $i$ is covered, $R \geq r_{i}$ must be satisfied, i.e., the served user must be located within the coverage region. This conditional expression is similar to (2.4), and consequently is equivalent to

$$
R \geq r_{i}-M_{2}\left(1-u_{i}\right)
$$

as in (2.6), where $M_{2}$ is a constant value which is sufficiently larger than the maximum possible value of $R$. Also, the first constraint in problem (2.7) becomes

$$
R^{2} \leq \Gamma(\alpha),
$$

where

$$
\Gamma(\alpha)=\frac{10^{\frac{\gamma-(A P(\alpha)+B)}{10}}}{\left(1+\alpha^{2}\right)},
$$

which enables us to omit the variable $h$ from (2.7), since $P(\alpha)$ is

$$
P(\alpha)=\frac{1}{1+a \exp (-b(\arctan (\alpha)-a))}
$$

by (2.1). Thus, (2.7) becomes

$$
\begin{array}{lll}
\underset{x_{D}, y_{D},\left\{u_{i}\right\}, R, \alpha}{\operatorname{maximize}} & \sum_{i \in \mathbb{U}} u_{i} & \\
\text { subject to } & R^{2} \leq \Gamma(\alpha)+M_{1}\left(1-u_{i}\right), & \forall i=1, \ldots,|\mathbb{U}|, \\
& R \geq r_{i}-M_{2}\left(1-u_{i}\right), \quad \forall i=1, \ldots,|\mathbb{U}|, \\
& x_{l} \leq x_{D} \leq x_{u}, & \\
& y_{l} \leq y_{D} \leq y_{u}, &
\end{array}
$$




$$
\begin{aligned}
& R \geq 0, \\
& u_{i} \in\{0,1\},
\end{aligned}
$$

$$
\forall i=1, \ldots,|\mathbb{U}|
$$

Note that if $\alpha$ and $R$ are known, $h$ can be evaluated by using (2.8). Since the variable $\alpha$ appears only in the right-hand-side of the first constraint of (2.13), the optimum value of $\alpha$, which maximizes $\Gamma(\alpha)$, maximizes the size of the feasible set of (2.13). Next, we numerically show that $\Gamma(\alpha)$ has only one local maxima. Hence, there exists a certain value, $\alpha^{*}$ that maximizes $\Gamma(\alpha)$.

Conjecture 1. For any $Q o S$ requirement, $\gamma$, and for any operating frequency, $f_{c}$, if a local maxima exists in the function $\Gamma(\alpha)$ defined in (2.11), then it is the only local maxima of the function for $\alpha \in[0, \infty]$ for the propagation environments whose parameters are listed in Table 2.1.

Observation. Observe that $\gamma$ and $B$ in (2.11) only scale the value of $\Gamma(\alpha)$. Since $B=$ $20 \log \left(\frac{4 \pi f_{c}}{c}\right)+\eta_{\mathrm{NLOS}}$, it also follows that the behaviour of $\Gamma(\alpha)$ does not depend on $f_{c}$. In other words, the maximum point, $\alpha^{*}$, does not change for different $\gamma$ and $f_{c}$, but the value of $\Gamma\left(\alpha^{*}\right)$ is scaled.

The behaviour of $\Gamma(\alpha)$ is only determined by the environment parameters in $A$ and $P(\alpha)$. By numerically plotting (2.11) in Fig. 2.2, we show that for all environments there exists only one maximum value, which occurs at $\alpha^{*}$. Moreover, it is observed that the local maximas marked in Fig. 2.2 are the only maximas for all environments.

We can use the derivative of $\Gamma(\alpha)$ to find $\alpha^{*}$, which is the root of $\frac{d \Gamma(\alpha)}{d \alpha}$, that can be calculated as

$$
\frac{d \Gamma(\alpha)}{d \alpha}=-\frac{10^{\Lambda}}{\Omega \Delta^{2}}\left(2 \alpha \Delta^{2}+A b K(\Delta-1)\right),
$$

where $K=18 \frac{\log (10)}{10 \log (\mathrm{e})}$, and $\Delta, \Lambda$ and $\Omega$ are

$$
\begin{gathered}
\Delta=\left(a \exp \left(b\left(a-\frac{180}{\pi} \arctan (\alpha)\right)\right)+1\right), \\
\Lambda=\frac{1}{10}\left(\gamma-B-\frac{A}{\Delta}\right), \\
\Omega=\left(\alpha^{2}+1\right)^{2} .
\end{gathered}
$$




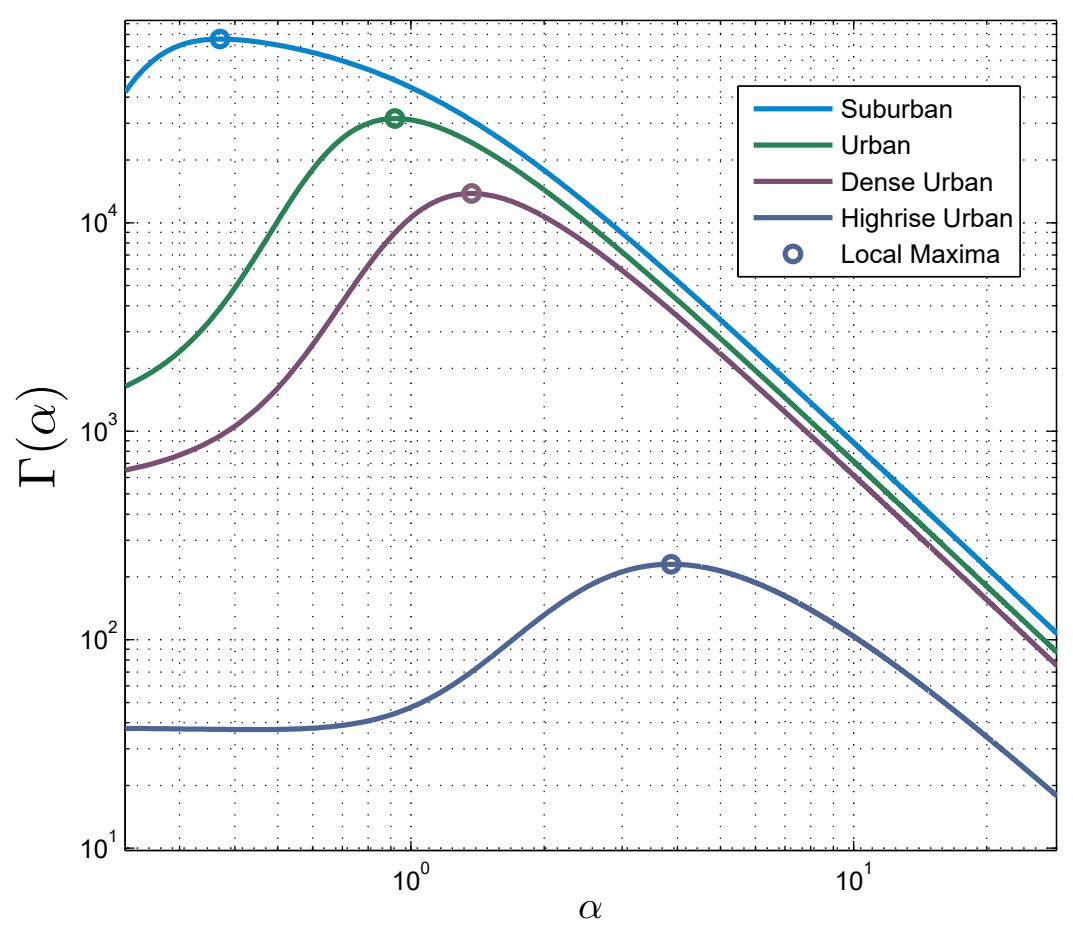

Figure 2.2: $\Gamma(\alpha)$ versus $\alpha$ for various environments.

Finally, we proceed with the bisection search to find the root of $\frac{d \Gamma(\alpha)}{d \alpha}$, which is $\alpha^{*}$. Note that, $\Delta$ yields that the maximum value of $\alpha^{*}$ can be $\tan \left(90^{\circ}\right)$. Also, the minimum value of $\alpha^{*}$ is 0 , because $\alpha^{*}$ is a ratio of positive quantities. The bisection search algorithm with a maximum number of iterations, $N_{u}$, and tolerance, $\epsilon$, can be summarized in Algorithm 1 .

After evaluating $\alpha^{*}$ using Algorithm 1, the problem given by (2.13) becomes MINLP, and can be solved to find $x_{D}, y_{D},\left\{u_{i}\right\}$, and $R$ by interior point optimizer of MOSEK solver.

\subsubsection{Numerical Results of Efficient 3-D Placement Method}

The numerical values of the parameters of the air-to-ground channels for different environments are calculated based on [28] and [129], and presented in Table 2.1. Also, all simulation parameters are provided in Table 2.2. It is assumed that the drone-BS has enough capacity to serve all the users in the coverage region. The effect of different environments is shown in Fig. 2.3 for 25 users by using $\gamma_{2}$. After finding $\alpha^{*}$ by using Algorithm 1, the 


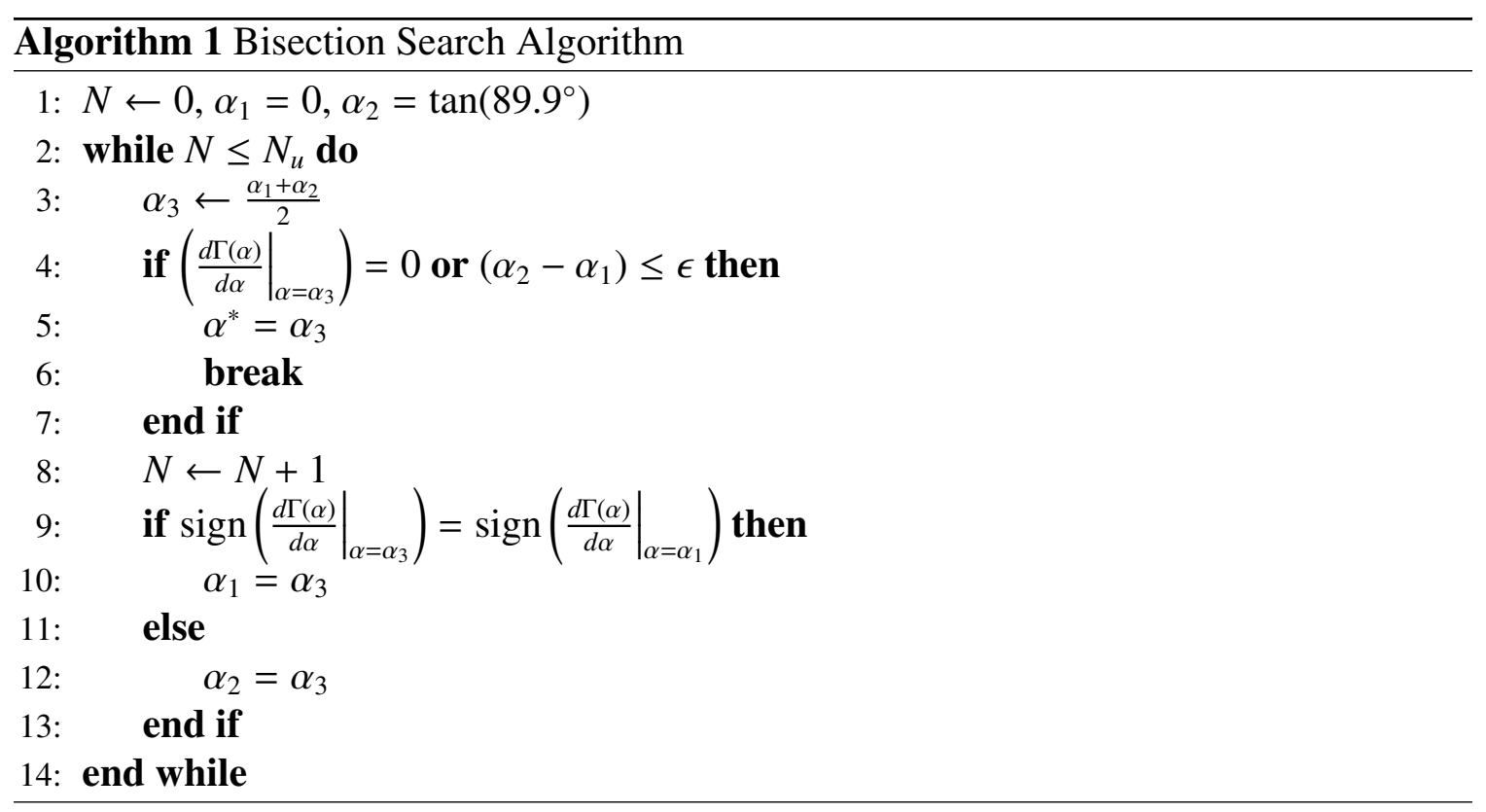

Table 2.1: RF Propagation parameters of different environments

\begin{tabular}{|c|c|}
\hline Environment & Parameters $\left(a, b, \eta_{\mathbf{L o S}}, \eta_{\mathbf{N L o S}}\right)$ \\
\hline Suburban & $(4.88,0.43,0.1,21)$ \\
\hline Urban & $(9.61,0.16,1,20)$ \\
\hline Dense Urban & $(12.08,0.11,1.6,23)$ \\
\hline High-rise Urban & $(27.23,0.08,2.3,34)$ \\
\hline
\end{tabular}

solution of the optimization problem in (2.13) yields $R$, which determines the size of the circular coverage region (i.e., drone-cell), and the location of the drone-BS in 2-D space, as shown with an asterisk in the corresponding color for each environment in Fig. 2.3. Note that not only the size of the region, but also the location of the drone-BS changes. Observe that there are users (some of them are pointed by arrows) right on the edge of the coverage region, which means that the altitude is determined efficiently such that there is no area wasted. As expected, the area covered by the suburban environment has the largest size, due to the reduced blockage compared to other environments. On the other hand, the highrise urban environment has the worst coverage. In fact, the significantly bad coverage in the high-rise urban environment should not mislead the reader to think that drone-BSs are not useful in dense urban areas. There are two fundamental reasons for the bad performance results in this study: 
Table 2.2: Simulation Parameters

\begin{tabular}{|c|c|}
\hline Parameter & Value \\
\hline$\left(x_{l}, x_{u}\right)$ & $(-1450,1450) \mathrm{m}$ \\
\hline$\left(y_{l}, y_{u}\right)$ & $(-1258,1258) \mathrm{m}$ \\
\hline$\left(\gamma_{1}, \gamma_{2}, \gamma_{3}\right)$ & $(90,100,125) \mathrm{dB}$ \\
\hline$f_{c}$ & $2.5 \mathrm{GHz}$ \\
\hline$N_{u}$ & 100 \\
\hline$\epsilon$ & $10^{-5}$ \\
\hline Monte Carlo Runs & 100 \\
\hline
\end{tabular}

- The user density is normally much higher in high-rise and dense urban environments compared to suburban and urban environments. Therefore, even a smaller coverage area can provide sufficient network performance gains, e.g., in terms of number of covered users. Note that the coverage issues in environments with high user density is not due to signal strength etc., but mainly because of the lack of capacity to serve the demand.

- The channel model utilized in this study has insufficiency in modelling air-to-ground channels in high-rise urban environments, becuase the model depends on average path loss. However, urban canyons can have significantly different path loss characteristics, and utilizing more information about the environment can lead to more precise channel models. In fact, this observation lead the authors to investigate airto-ground channels in modern urban environments in Chapter 3.

To elaborate more on the effect of environment parameters and the performance of the algorithm, we show the average revenue for varying QoS requirements in different environments together with 95\% confidence interval for the revenue in Fig. 2.4. The results are obtained by using 100 Monte Carlo simulations. In each simulation, 40 users are generated randomly in the cell according to a uniform probability distribution. The results show that the number of served users varies by at most 1 user. Hence, the performance of the proposed method is consistent. The parameter $\gamma_{3}$ provides the maximum revenue by enabling a coverage area larger than the size of the macrocell, because we are allowing a pathloss of $125 \mathrm{~dB}$ for the user to be served. Note that the average revenue for the high-rise urban 


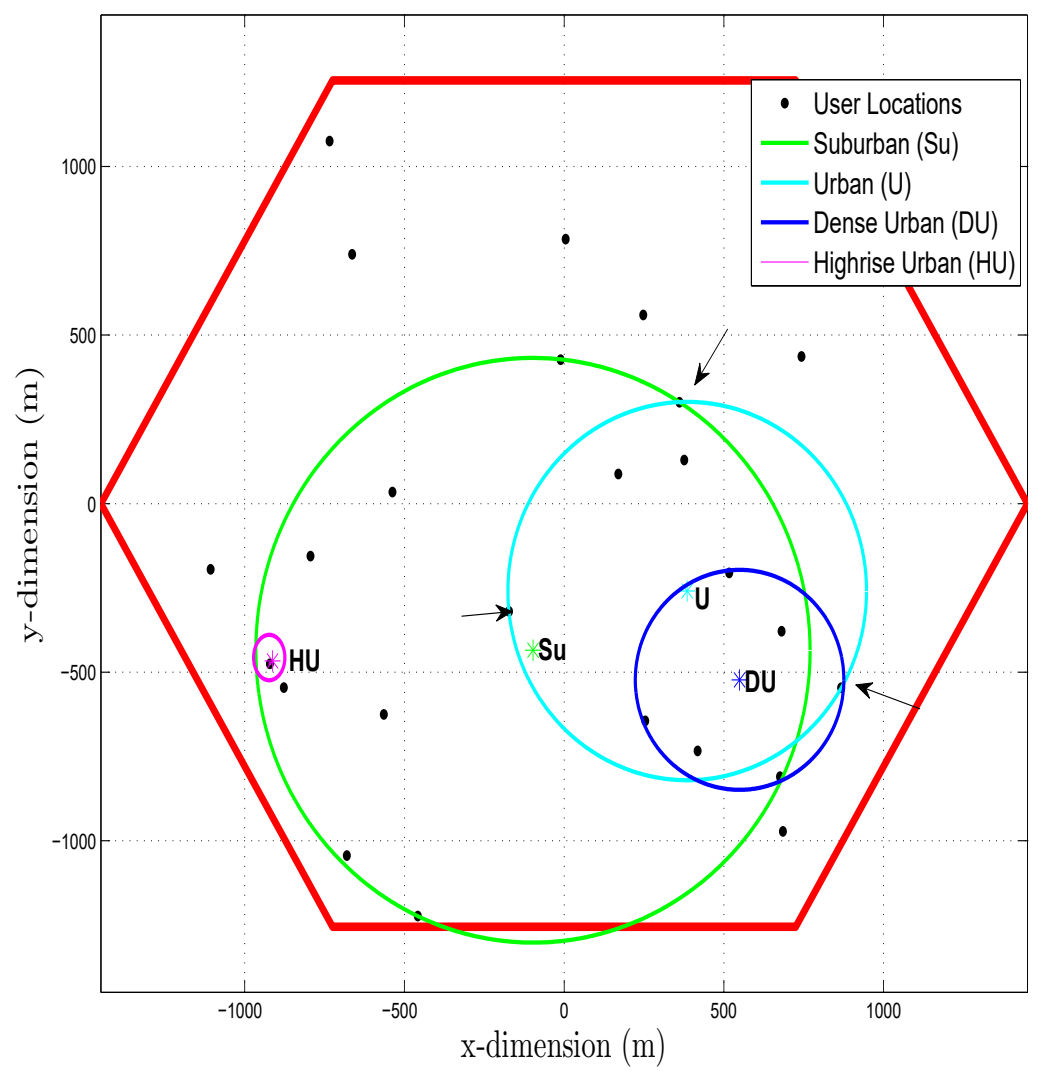

Figure 2.3: Effect of environment on the location and size of the coverage area (circles in the figure) is shown.

environment is significantly worse than the other environments for this sparse user distribution. The dramatic drop of revenue can be understood by comparing the parameters of suburban and high-rise urban environments. For instance, $\eta_{\mathrm{NLoS}}$ increases by $13 \mathrm{~dB}$ for the high-rise environment, which alone can reduce the coverage area by more than 100 times. Considering the changes in the other parameters, the significant reduction in the coverage area, and accordingly revenue, is not surprising. However, more users could be covered if the users were in proximity to each other, i.e., clustered. Hence, measuring traffic characteristics in space, such as the amount of clustering as shown in [130], can be of significant importance for determining the efficiency of drone-BS assistance for cellular networks. 


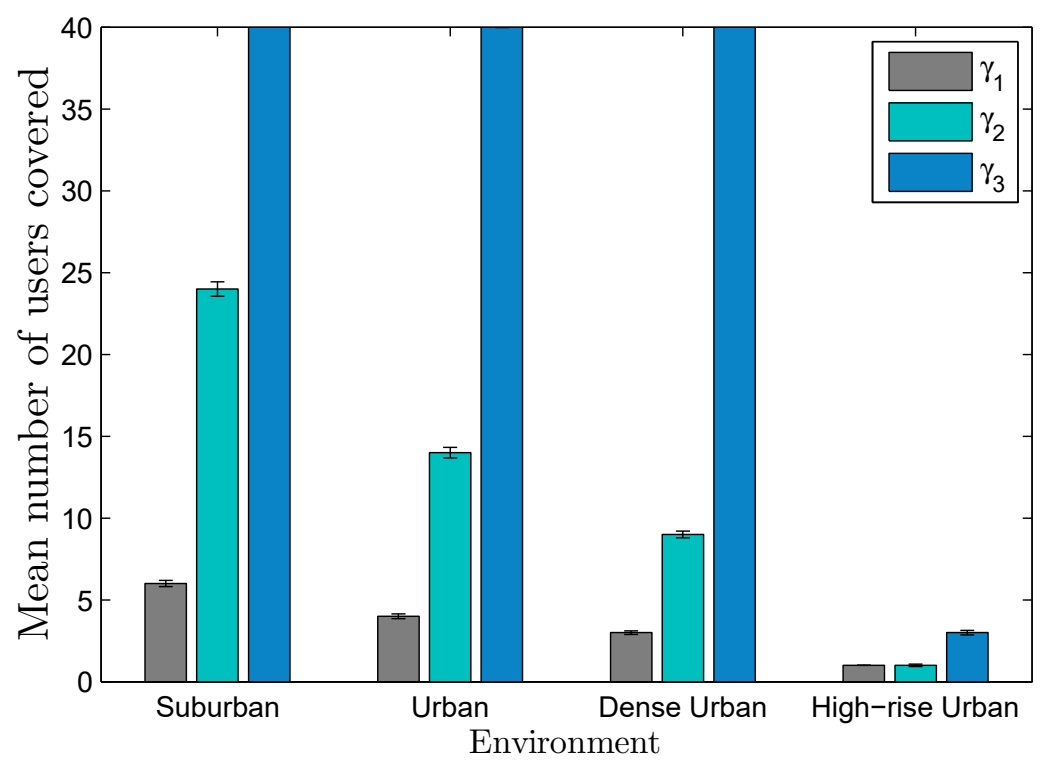

Figure 2.4: Mean number of users covered in different environments with $95 \%$ confidence interval. 40 users are distributed uniformly in one macrocell.

\subsection{Efficient 3-D Placement for a MmWave-drone-BS}

Despite a number of research works on drone-BS deployment, the specifics of mmWavebased drone-assisted communication has remained insufficiently studied so far [131-133]. Operating in extremely high frequency (EHF) bands and having larger bandwidths at its disposal, mmWave radio technology is being shaped as the 5G New Radio [134]. Along with their benefits, mmWave systems are facing many challenges. One of these is shorter wavelengths for which smaller objects, such as humans, become obstacles for the line-ofsight (LoS) radio propagation [135,136]. Hence, it is crucial to account for the human body blockage when evaluating the performance or planning the deployment of mmWave-BSs. In contrast to lower frequencies, another challenge at mmWave bands is that the path loss (PL) increases significantly with the growing distance from a transmitter (Tx) to a receiver (Rx) [137]. Hence, there is a trade-off between placing a drone at a higher altitude (which would provide better LoS links) and keeping the PL minimal (which increases with the growing distance). As a result, efficient 3-D placement methods are needed for mmWavedrone-BSas well.

There are several important benefits that motivate the utilization of mmWave-based 
drone-BSs, particularly for the temporary and spontaneous events, as described below:

- Able to arrive at the crowded location quickly, drones equipped with wireless access capabilities help operators serve events, where traffic demand becomes higher than expected for a certain period of time, but where it is not feasible to deploy a static network infrastructure to serve such amounts of data on a regular basis.

- Even though higher altitudes lead to larger probabilities to maintain the LoS link, they also increase the 3-D distance, thus making the PL higher. Therefore, an optimal altitude, which minimizes the PL and maximizes the LoS probability, may exist. While the terrestrial infrastructure cannot alter the height of the BSs quickly in order to improve the signal quality, the flexibility of the drone-BSs offers an opportunity to place them over the crowd and adjust their height when needed.

- To achieve $100 \mathrm{Mbit} / \mathrm{s}$ per user expected of the $5 \mathrm{G}$ systems, mmWave communication is an appropriate solution whereas the conventional infrastructure will need a significant number of cellular BSs to support the required data rate, which leads to severe interference. The latter could be shown using simple analysis where the link capacity for the cell edge-user over mmWave with the carrier of $28 \mathrm{GHz}$ and the conventional microwave cellular link with the carrier of $2.1 \mathrm{GHz}$ is calculated as $r(x)=B_{u} \log [1+S(x)]$. Here, $B_{u}$ is the bandwidth available to the user of interest and $S(x)$ is the average signal-to-noise ratio (SNR) for this user at the cell edge of radius $x$. For the same number of active users, the cellular link with the maximum available bandwidth (B) of $20 \mathrm{MHz}$ delivers about 10 times lower data rates than what mmWave ( $\mathrm{B}=1 \mathrm{GHz}$ ) does, even in ideal conditions where no interference is assumed. In an optimistic case, to provide the average data rate of $100 \mathrm{Mbit} / \mathrm{s}$ per user, for a cell having $50 \mathrm{~m}$ radius and 70 users, one mmWave-BS is sufficient, whereas the required number of the conventional BSs is 10 times higher. Therefore, the larger bandwidth of mmWave-BSs accentuates the utilization of those to support the mass events and mitigate the growth of interference to deliver the 5G data rates [138].

Although there are several strong motivators to utilize mmWave-drone-BSs as described above, the difficulties of mmWave communications should be considered. Note that some reasons for potentially bad performance of mmWave communication, such as 
inclement weather conditions, e.g., rain, and dense foliage, cannot be mitigated via the mobility of a drone-BS. On the other hand, we investigate efficient deployment of a mmWavedrone-BS by taking into account that a LoS link may be blocked by a human body, which is a unique property of mmWave communications. Having in mind that the height of the mmWave-drone-BS is comparable with the height of the BSs mounted on the walls of the buildings, and assuming quasi-stationary drones hovering at a certain altitude [28], we approximate the air-to-ground channel model with the terrestrial channel model [139] for the sake of our first-order analysis. Note that there is not yet an air-to-ground mmWave channel model that is well-investigated and available.

\subsubsection{System Model for MmWave-drone-BS Placement}

We assume that there is a spontaneous and temporary mass event, such as a rural fair, which does not comply with the existing infrastructure and planned capacity of the operators in

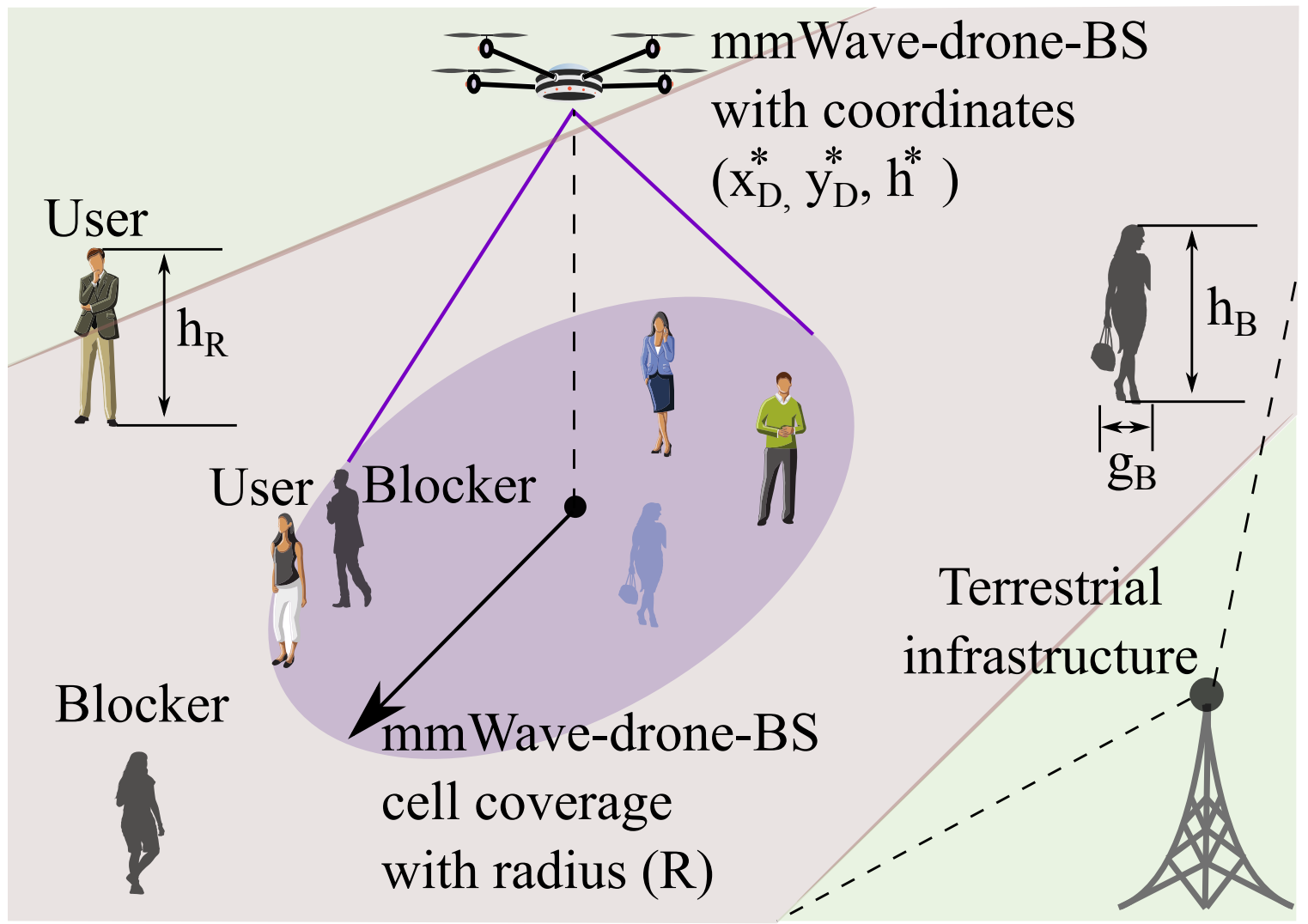

Figure 2.5: Target scenario with mmWave-drone-BS, users, and blockers. 
that area. Consider a set of identical users, $\mathbb{M}$, which are distributed randomly in the area of interest as illustrated in Fig. 2.5. Due to lack of capacity, the operator is incapable of serving all the users at the fair. Hence, we consider the assistance of a mmWave-drone-BS to inject capacity across space and time. The mmWave-drone-BS is integrated into the current infrastructure via a dedicated long range backhaul channel over a different frequency [140].

Inspired by the adoption of terrestrial channel models for air-to-ground channels of quasi-stationary drone-BSs [28,31,141], we employ the model in [142] for the first-order analysis of mmWave-drone-BSs. There are two motivations for choosing a terrestrial channel model: First, contemporary drone-BSs with a rotary wing [143] can be made as stationary as cell towers, especially under mild weather conditions. Second, the short range of mmWave links prevents from using high-altitude drones due to the inherently high PL with increasing distance between the Tx and Rx. Therefore, the altitude of a mmWave-droneBS must be comparable with the altitude of the static mmWave-BSs deployed on the walls, lamp posts, etc. For the sake of our analysis, the small scale fluctuations in the environment are neglected as proposed in [28].

The considered scenario consists of the mmWave-drone-BS located at height $h_{D}$ and human blockers modeled as cylinders with the average height of $h_{B}$ and the average diameter of $g_{B}$. For a snapshot analysis, assume a Poisson field of static human blockers with the density of $\lambda$, where $|\mathbb{M}|$ humans are distributed across the area $S$ with the parameter $\lambda S$, and $|\cdot|$ indicates the cardinality of a set. Note that all users are considered as blockers for each other. The user terminal is assumed to be located at the height $h_{R}$, where $h_{R}<h_{B}$, since the terminal carried by a human is usually lower than the height of the human itself. Hence, if the user $i$ is communicating with the mmWave-drone-BS, then all other users/humans in the coverage area $\mathcal{A}$ with radius $R$ are blockers, if their heights are large enough to block the LoS between Rx and Tx. Note that the coverage radius $R$ depends on the ability of droneBS to support on average the minimum quality-of-service (QoS) experienced by the cell edge user; therefore, it is highly affected by the height of the drone-BS and the probability of $\operatorname{LoS}$ as will be shown later.

Following [144], we assume that radio interference does not have a major effect, which is a common assumption for most mmWave-based systems with highly directional antennas, and that the system under study is noise-limited.

Recall that the PL models for LoS and NLoS links at mmWave frequencies follow [139] 
Table 2.3: Notation and parameters

\begin{tabular}{|c|c|}
\hline Parameter & Description \\
\hline$S$ & Area of interest \\
\hline$h_{D}, h_{R}, h_{B}$ & $\begin{array}{l}\text { Height of drone-BS, Rx, and human } \\
\text { blockers }\end{array}$ \\
\hline$R$ & Cell radius of the drone-BS \\
\hline$e_{i}, r_{i}$ & $\begin{array}{l}\text { 3-D, 2D distance between drone-BS and } \\
i^{\text {th }} \mathrm{Rx}\end{array}$ \\
\hline$g_{B}$ & Diameter of human blockers \\
\hline$\lambda$ & Density of human blockers \\
\hline $\mathbb{P}_{L}$ & Probability of LoS \\
\hline$L_{L, i}, L_{N, i}$ & Path loss for LoS/NLoS $i^{\text {th }} \mathrm{Rx}$ \\
\hline$L_{a, i}$ & Average path loss for $i^{\text {th }} \mathrm{Rx}$ \\
\hline$|\mathbb{M}|$ & $\begin{array}{l}\text { Total number of humans in the area of in- } \\
\text { terest }\end{array}$ \\
\hline$h^{*}$ & Optimal height of the drone-BS \\
\hline$\left(x_{D}^{*}, y_{D}^{*}\right)$ & Optimal 2D position of the drone-BS \\
\hline$N$ & $\begin{array}{l}\text { Maximum number of users served by one } \\
\text { drone-BS }\end{array}$ \\
\hline$Q, \sigma_{i}$ & Target SNR, SNR for $i^{\text {th }}$ Rx \\
\hline$\gamma$ & Maximum tolerable path loss \\
\hline
\end{tabular}

and are given as

$$
\begin{gathered}
L_{L, i}=\alpha_{L}+10 \beta_{L} \log _{10}\left(d_{i}\right), \\
L_{N, i}=\alpha_{N}+10 \beta_{N} \log _{10}\left(d_{i}\right),
\end{gathered}
$$

where $\alpha_{L}, \beta_{L}, \alpha_{N}$, and $\beta_{N}$ are the parameters of the LoS and NLoS PL models, and $e_{i}=$ $\sqrt{\left(x_{i}-x_{D}\right)^{2}+\left(y_{i}-y_{D}\right)^{2}+\left(h_{D}-h_{R}\right)^{2}}$ is the 3-D distance between the drone-BS and Rx.

In order to account for the human body blockage, we adopt the probability of LoS, $\mathbb{P}_{L}$, for a user $i$ from [142] by modifying it in the case of the constant height and diameter of blockers for further analytic tractability as

$$
\mathbb{P}_{L}\left(r_{i}, h_{D}\right)=\exp \left(-\lambda g_{B} \frac{r_{i}\left(h_{B}-h_{R}\right)}{\left(h_{D}-h_{R}\right)}\right),
$$

where $r_{i}$ is $2 \mathrm{D}$ distance between drone-BS and Rx. Then, the average PL for the cell edge 
user $i$, located at distance $R$ from Tx, becomes

$$
L_{a, i}=\mathbb{P}_{L}\left(R, h_{D}\right) L_{L, i}+\left[1-\mathbb{P}_{L}\left(R, h_{D}\right)\right] L_{N, i} .
$$

As one may find in [28] and similar works, the average value of PL is sufficient to perform the first-order analysis. Since the random behavior with the corresponding distribution is not the focus of this study, the distributions of fading and shadowing are disregarded. As it was observed in [142], there exists the optimal height of the Tx, where the average PL assumes its minimum value.

\subsubsection{MmWave-drone-BS Deployment Method}

In order to support the current cellular infrastructure and provide higher data rates for every user in the area, the aim is to offload as many users as possible to the mmWave-droneBS. Because the users are randomly distributed in the region, the area to be covered by a mmWave-drone-BS (drone-cell coverage) and the altitude of the drone-BS are not known a priori.

On the one hand, deploying a mmWave-drone-BSs at a higher altitude leads to the greater LoS probability as can be observed from (2.19). On the other hand, mmWavedrone-BSs are energy critical devices and higher altitudes may require more transmission power due to increased distance between the users and the drone-BSs to compensate for larger PL. Therefore, the objective of covering the maximum number of users with minimum energy means the smallest area enclosing the highest number of users, while the minimum height that can provide coverage over that area must be derived.

As observed in (2.19) and (2.20), the average PL depends on the altitude of the Tx, as well as the horizontal distance between the Tx and Rx. Therefore, the optimal placement of a mmWave-drone-BS involves all dimensions, namely, the optimal position is $\left(x_{D}{ }^{*}, y_{D}{ }^{*}, h^{*}\right)$. Fixing the horizontal location of the drone-BS and searching for $h^{*}$ to provide the maximum number of users to be covered ( $1 D$ search), or fixing the altitude and searching for $\left(x_{D}{ }^{*}, y_{D}{ }^{*}\right)$ ( $2 D$ search) may not result in the most effective deployment. The search for the optimal position of a mmWave-drone-BS must thus be performed in 3-D. Not only the expansion of the search space to 3-D makes it very hard to conduct an exhaustive search, but also (2.20) 
is analytically difficult. Therefore, in this section, we propose an efficient 3-D placement method for mmWave-drone-BSs.

The problem to find $\left(x_{D}^{*}, y_{D}^{*}, h^{*}\right)$ can be formulated as

$$
\begin{array}{ll}
\operatorname{maximize}_{\left.x_{D}, y_{D}, h, m_{i}\right\}} \sum_{i \in \mathbb{M}} m_{i} & \\
\text { subject to } & \\
m_{i} \sigma_{i} \geq Q, & \forall i=1, \ldots,|\mathbb{M}|, \\
\sum_{i \in \mathbb{M}} m_{i} \leq N, & \\
x_{l} \leq x_{D} \leq x_{u}, & \\
y_{l} \leq y_{D} \leq y_{u}, & \\
h_{l} \leq h \leq h_{u}, & \forall i=1, \ldots,|\mathbb{M}|, \\
m_{i} \in\{0,1\}, & \mathbb{M} \mid,
\end{array}
$$

where $m_{i}$ is a binary variable indicating whether the $i^{t h}$ user of the set $\mathbb{M}$ is covered (1) or not (0), $x_{D}, y_{D}$ are the possible coordinates of the drone-BS, $h=h_{D}-h_{R}$, and $\sigma_{i}$ is the SNR for the user $i$. Then, $Q$ and $N$ represent the target SNR level for the served user $i$ and the capacity of the drone-BS in terms of the maximum number of users that it can serve, respectively. The upper and the lower limits of the available positions across all three dimensions are indicated by the subscripts $u$ and $l$, correspondingly. While (2.21b) determines which users can be served, (2.21c) captures the maximum number of the served users.

Apart from the antenna gains, transmit power, etc., the maximum tolerable PL for the $i^{\text {th }}$ user, $\gamma$, corresponds to the target SNR of the $i^{\text {th }}$ user, $Q$. Hence, using (2.20), (2.21b) becomes $m_{i} L_{a, i} \leq \gamma$. Note that our approach is not limited to the model in [139], and other channel models may be considered as well. After further derivations, the QoS depicted in (2.21b) can be represented in terms of distance between user $i$ and the drone-BS as

$$
r_{i}^{2}+h^{2} \leq 10^{\left[2 \tilde{\gamma}+\mathbb{P}_{L}(R, h) k_{2}\right] /\left[\mathbb{P}_{L}(R, h) k_{3}+k_{4}\right]}
$$

where $r_{i}=\sqrt{\left(x_{i}-x_{D}\right)^{2}+\left(y_{i}-y_{D}\right)^{2}}$ is $2 \mathrm{D}$ distance between user $i$ and the drone-BS, $\tilde{\gamma}=$ $\gamma-\alpha_{N}, k_{2}=\alpha_{N}-\alpha_{L}, k_{3}=10\left(\beta_{L}-\beta_{N}\right), k_{4}=10 \beta_{N}$, whereas $R$ is the coverage radius of 
the drone-BS. Note that any user with the horizontal distance of less than $R$ will be served, since its minimum SNR requirements at the cell edge are satisfied on average. Furthermore, introducing the variable $\omega=R / h$ and expressing $\mathbb{P}_{L}(R, h)$ in terms of $\omega,(2.21 \mathrm{~b})$ becomes

$$
r_{i}^{2} \leq \Gamma(\omega)
$$

where $\Gamma(\omega)$ is the following

$$
\Gamma(\omega)=\frac{10^{\left(2 \tilde{\gamma}+k_{2} \mathrm{e}^{\omega k_{1}}\right) /\left(k_{3} \mathrm{e}^{\omega k_{1}}+k_{4}\right)}}{1+\frac{1}{\omega^{2}}},
$$

where $k_{1}=-\lambda g_{B}\left(h_{B}-h_{R}\right), h_{B}>h_{R}$.

Proposition 1. The function $\Gamma(\omega)$ has the maximum point $\omega^{*}$, which is considered to be optimal.

Proof. To find the maximum point, we first need to establish an extremum point of $\Gamma(\omega)$, by taking a derivative, equating it to zero, and solving the following

$$
\begin{aligned}
& k_{1} e^{\omega k_{1}}\left(\omega^{3}+\omega\right)\left(k_{2} k_{4}-k_{3} \tilde{\gamma}\right) \ln (10) \\
& +\left(e^{\omega k_{1}} k_{3}+k_{4}\right)^{2}=0
\end{aligned}
$$

Note that the above always has a solution for $\beta_{L}<\beta_{N}$ and $h_{B}>h_{R}$. It could be solved numerically and offers the extremum point, $\omega^{*}$. By taking the second derivative of $(2.25)$ and obtaining the negative value at the extremum point $\omega^{*}$, we establish that $\omega^{*}$ is also the maximum of $\Gamma(\omega)$.

As there is no closed form solution to find $\omega^{*}$, it is important to show the uniqueness of this point, which is formally proven in Section 3.3.1.

The optimal value, $\Gamma\left(\omega^{*}\right)$, can be inserted into (2.23). The resulting optimization problem is then

$$
\underset{x_{D}, y_{D},\left\{m_{i}\right\}}{\operatorname{maximize}} \sum_{i \in \mathbb{M}} m_{i}
$$

subject to 


$$
\begin{array}{ll}
r_{i} \leq \sqrt{\Gamma\left(\omega^{*}\right)}+K\left(1-m_{i}\right), & \forall i=1, \ldots,|\mathbb{M}|, \\
x_{l} \leq x_{D} \leq x_{u}, & \\
y_{l} \leq y_{D} \leq y_{u}, & \\
m_{i} \in\{0,1\}, & \forall i=1, \ldots,|\mathbb{M}|,
\end{array}
$$

where $K$ is a large enough value [31]. Once $x_{D}^{*}$ and $y_{D}^{*}$ are obtained, $R$ can be calculated by identifying the user at the drone-cell edge, i.e., $\max _{m_{i} \in \mathbb{M}}\left(r_{i} \mid m_{i}=1\right)$. Then, $h^{*}$ can be derived by using $\omega^{*}$.

Moreover, the optimal height can also be produced directly from (2.20) by taking a derivative of the average PL. Note that in this case, the cell coverage $R$ should be known beforehand. In this study, we propose an approach to numerically establish the optimal height of Tx, $h^{*}$, by solving the following

$$
\begin{aligned}
& -C\left[\alpha_{L}-\alpha_{N}\right]\left[\left(h^{*}-h_{R}\right)^{2}+R^{2}\right] e^{\frac{C}{h^{*}-h_{R}}} \\
& +10 C\left[\beta_{N}-\beta_{L}\right] \log _{10}\left(\sqrt{\left(h^{*}-h_{R}\right)^{2}+R^{2}}\right) \\
& +\frac{10\left[\beta_{L}-\beta_{N}\right]\left[h^{*}-h_{R}\right]^{3}}{\ln (10)} e^{\frac{C}{h^{*}-h_{R}}}+10 \beta_{N}=0,
\end{aligned}
$$

where the auxiliary variable $C=-\lambda g_{B} R\left(h_{D}-h_{R}\right)$.

The above 3-D placement problem can be solved by using e.g., interior-point optimization method via MOSEK [145], both efficiently and accurately. Indeed, the efficient 3-D placement algorithm in (2.26a) offers the same result as in (2.27) for the same value of $R$ derived with our 3-D placement algorithm.

\subsubsection{Numerical Results and Discussions}

In this section, we illustrate representative numerical results produced for different human densities $\lambda$, where the humans are uniformly distributed within a $100 \times 100 \mathrm{~m}^{2}$ area. The parameters for the considered scenario are collected in Table 2.4. Our target is to serve the 


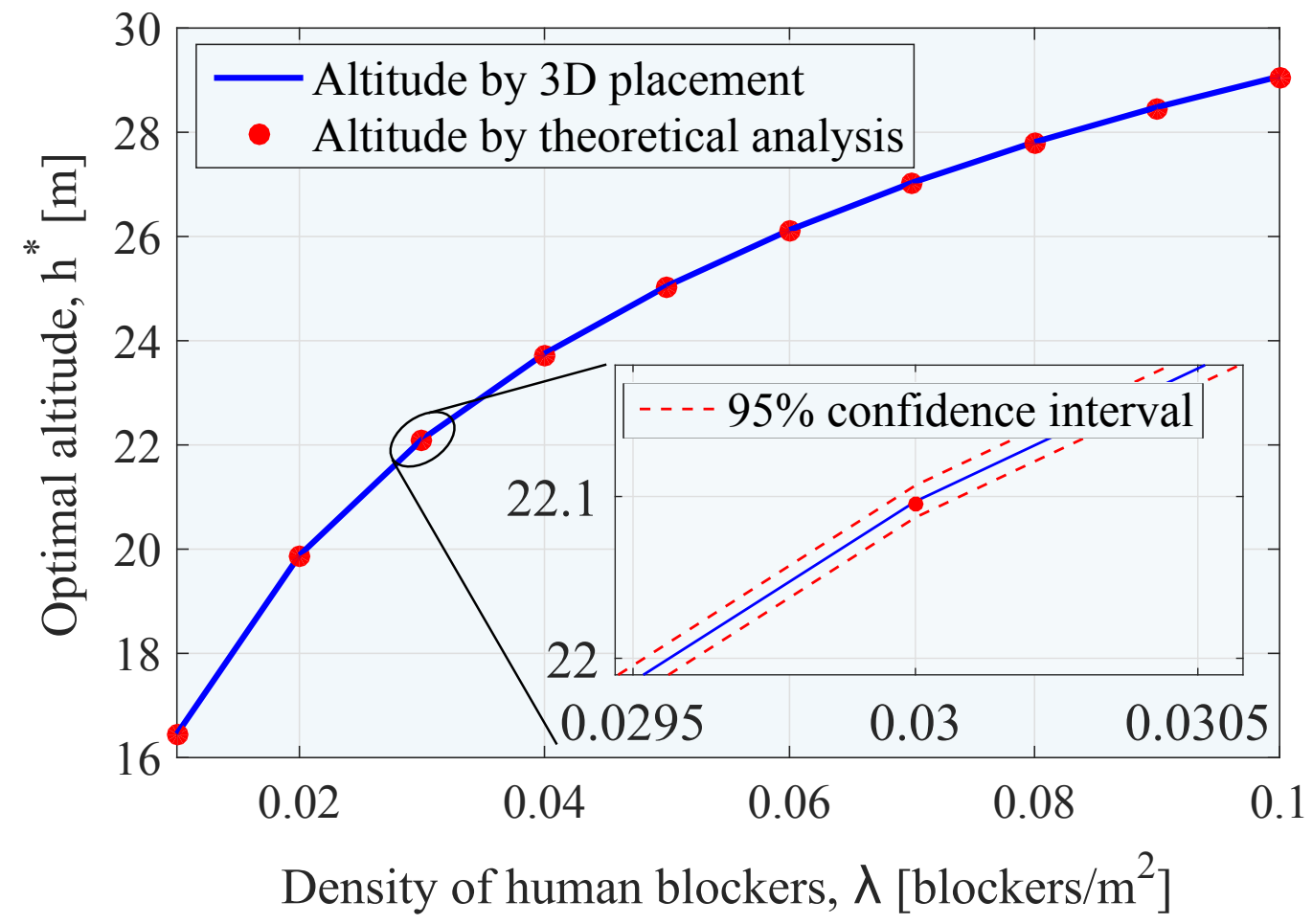

Figure 2.6: Comparison of the optimal altitude results from 3-D placement with theoretical analysis vs. density of blockers, $\lambda$.

maximum number of users from the set of total number of humans $|\mathbb{M}|$ with a mmWavedrone-BS. It should be noted that for every realization of the scenario the coordinates of the users as well as the total number $|\mathbb{M}|$ are known for the problem to solve. We set the maximum tolerable path loss, $\gamma$, equal to $110 \mathrm{~dB}$ based on the following assumed parameters: bandwidth is $1 \mathrm{GHz}, \mathrm{Rx}$ and Tx antenna gains are $5 \mathrm{~dB}$ and $10 \mathrm{~dB}$, respectively, Tx power is $20 \mathrm{dBm}$, noise figure is $6 \mathrm{~dB}$, and target $\mathrm{SNR}$ is $3 \mathrm{~dB}$. Also, $95 \%$ confidence interval is calculated for the entire set of runs to demonstrate the consistency of the proposed method. The following formula is used for confidence interval calculations: $\bar{x} \pm \mathrm{Z}_{a / 2} \times \frac{\sigma}{\sqrt{(n)}}$, where $\bar{x}$ is the mean, $\mathrm{Z}$ is the confidence coefficient, $a$ denotes the confidence interval, while $\sigma$ and $n$ represent the standard deviation and the sample size, respectively.

First, Fig. 2.6 demonstrates the behavior of the altitude of the mmWave-drone-BS as the density of blockers increases. We observe that the altitude becomes higher as the density grows. The reason is that higher altitude makes the probability of blockage lower but sacrifices the radius of the drone-BS coverage in order to reduce the 3-D distance, in order 


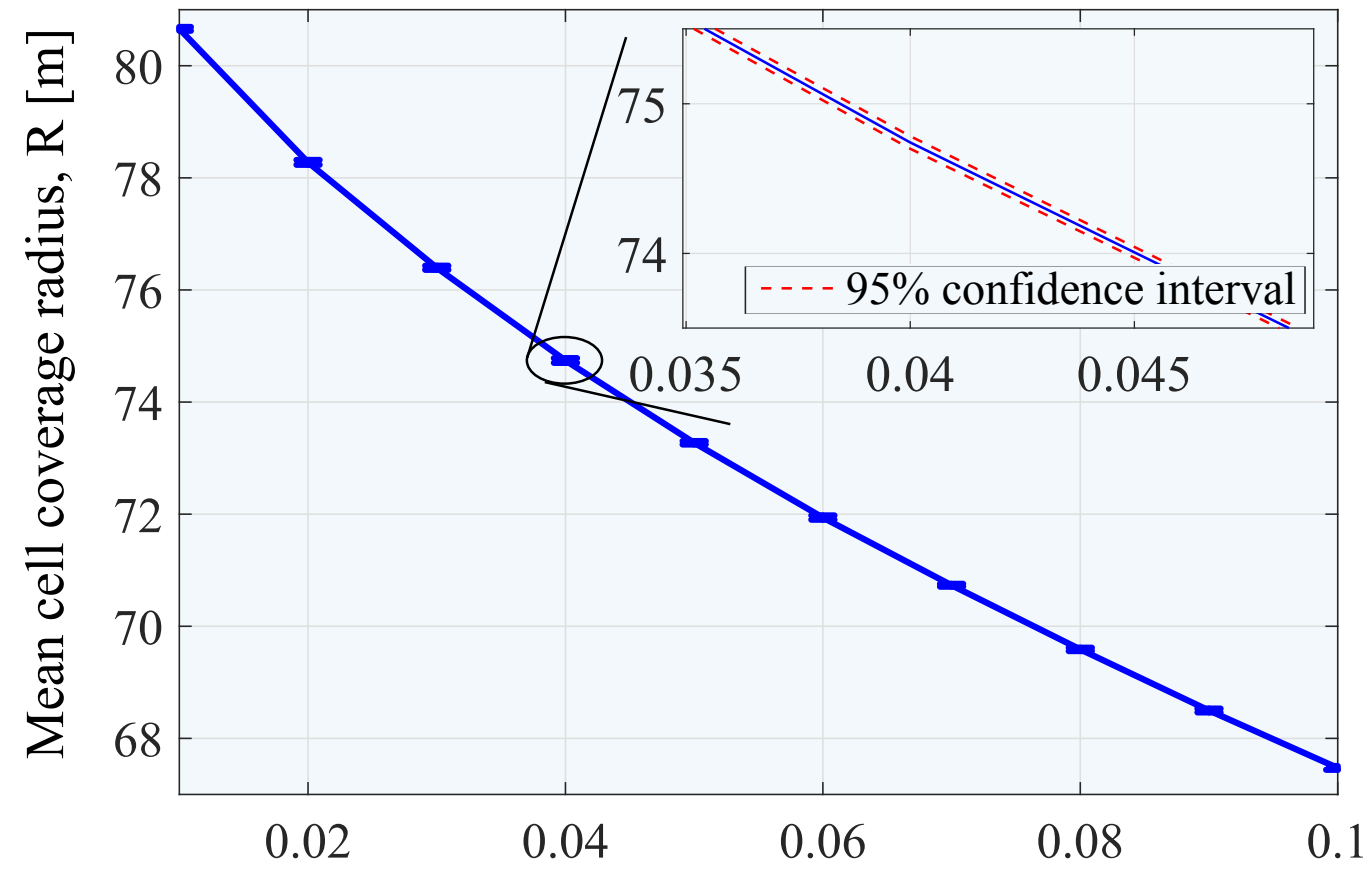

Density of human blockers, $\lambda\left[\right.$ blockers $\left./ \mathrm{m}^{2}\right]$

Figure 2.7: Radius, $\mathrm{R},(\mathrm{m})$ vs. density of blockers, $\lambda$, with error bar.

to satisfy the minimum SNR. This confirms the importance of appropriate height selection. In addition, the plot shows a comparison of the altitude by the 3-D placement with that derived from the theoretical result in (2.27). The analysis requires the cell coverage obtained with the 3-D placement in order to produce the height of the BS. The results indicate a reasonable match between the two. It should be noted that the proposed 3-D

Table 2.4: Baseline system parameters

\begin{tabular}{ll}
\hline Parameter & Value \\
\hline Height of Rx, $h_{R}$ & $1.3 \mathrm{~m}$ \\
Height of a human blocker, $h_{B}$ & $1.7 \mathrm{~m}$ \\
Diameter of a human blocker, $g_{B}$ & $0.5 \mathrm{~m}$ \\
Frequency band & $28 \mathrm{GHz}$ \\
LoS path loss model parameters & $\alpha_{L}=61.4, \beta_{L}=2$ \\
NLoS path loss model parameters & $\alpha_{N}=72, \beta_{N}=$ \\
& 2.92 \\
Maximum number of users served by & 100 \\
drone-BS, $N$ & \\
Area of interest, $S$ & $100 \times 100 \mathrm{~m}^{2}$ \\
\hline
\end{tabular}




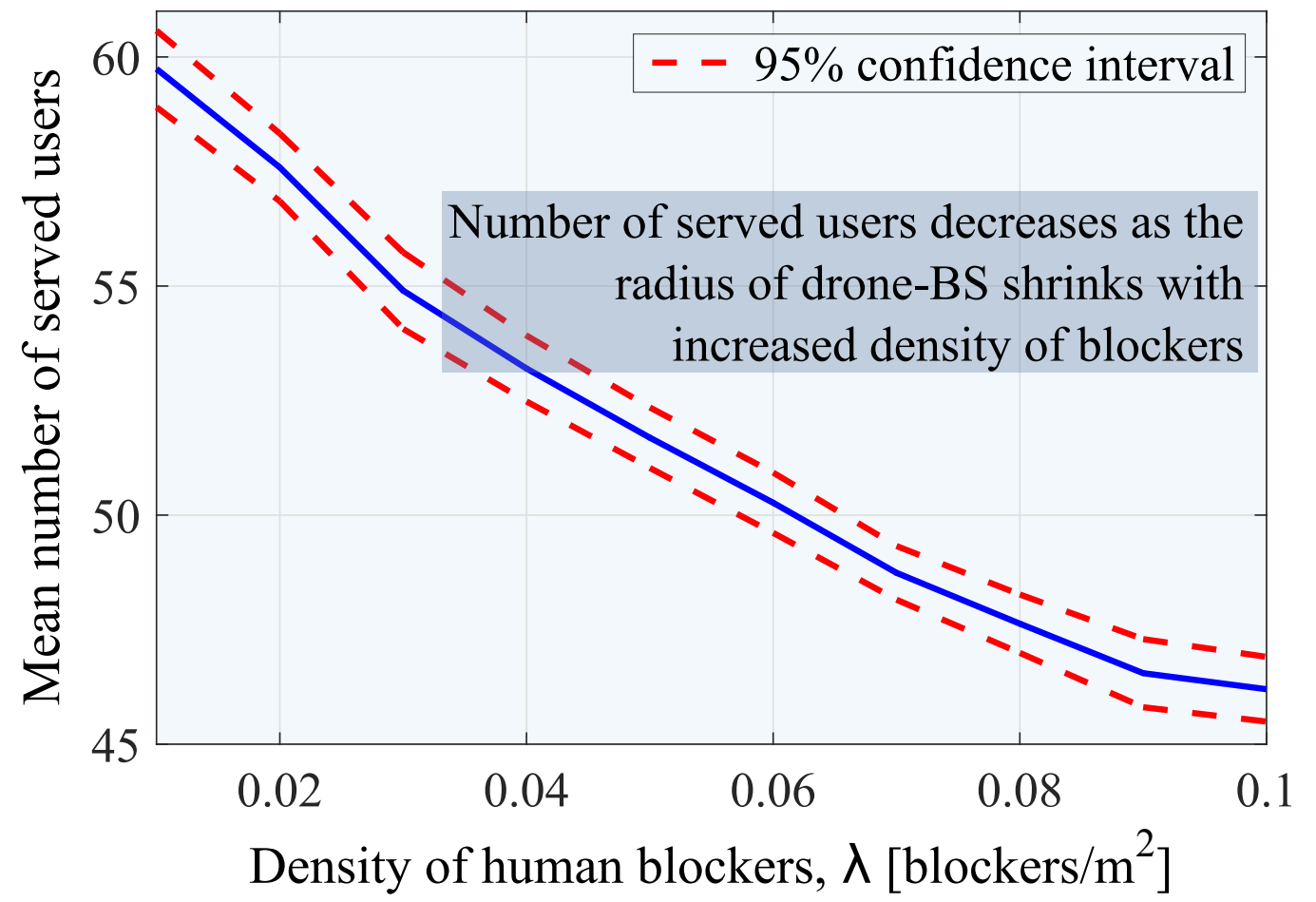

Figure 2.8: Number of users served by mmWave-drone-BS vs. density of blockers, $\lambda$.

placement provides the coordinates of the drone-BS, not only altitude but also the location in the horizontal plane, which allows for efficient drone-cell deployment in order to serve the maximum number of users.

In Fig. 2.7, the aforementioned relation between the mean value of the mmWave-droneBS cell coverage and the density of blockers is displayed. It is observed that the cell radius, $R$, decreases as the density grows. This could be explained by the fact that the probability of blockage becomes larger, thus yielding a higher altitude of the drone-BS and smaller cell radius to reduce 3-D distance in order to facilitate the satisfaction of the minimum SNR requirements.

Further, we consider the number of served users as illustrated in Fig. 2.8. We learn that the average number of users served by one drone-BS decreases as the density of blockers grows. This could be explained by the fact that the effective cell radius degrades as it was shown earlier. Therefore, the density of blockers highly affects the optimal height of the drone-BS, which then impacts the shrinking of the cell coverage, and finally the reduced number of served users. This implies the importance of considering all of the variables as 
they have a major effect on the load of the BS as well as its ability to satisfy the minimum QoS requirements.

\subsection{Environment-Aware Drone-Base-Station Placements in Modern Metropolitans}

In the preceding sections, we introduced drone-base-stations as moving base stations with wireless backhaul, and discussed several deployment scenarios and methods. Recall that drone-BSs are particularly useful when there is mismatch between the wireless capacity demand and available network resources. These mismatches may occur in under-utilized areas, as well as areas with dense populations. Among these cases, the latter one may be particularly important for wireless network operators, because population density ensures revenue, and proliferates potential applications, such as improving resilience of smart cities [116], or providing additional coverage [146], to name a few. Since drone-BSs are extremely flexible yet energy-critical devices, issues on their management, and especially drone-BS placement, has gained significant interest [2,40,52,112,116,146,147]. However, modelling of air-to-ground path loss characteristics is following these developments rather slowly, due to tedious experiments. On the other hand, drone-BSs are expensive to operate and tight on energy budget. Therefore, their precise operation and planning is critical. That requires sufficiently accurate channel models.

Among recent air-to-ground path loss models, [28] gained popularity due to the consideration of urban environments, and the ability to model the relationship between coverage and altitude of the drone-BS. However, in Section 2.1.3 we observe that [27] has limitations in modeling some urban environments, especially the high-rise urban environment. On the other hand, there is no other channel model for drone-BSs in the literature that can capture the 3-D communications characteristics in urban settings. The aim of the study in the chapter is to fill this gap temporarily with a channel model adopted from terrestrial models and shown to be suitable for drone-BSs via ray-tracing simulations. The key idea of the proposed channel model is utilizing the already available information on urban environment (e.g., via maps), such as orientations of streets.

This chapter begins with describing the urban environment that is considered for the 
channel modeling simulations. Then, we introduce the proposed air-to-ground channel model, and compare it with ray tracing simulations and the widely used model in [27]. We provide a more detailed investigation with simulation results and discussions. Finally, we provide a summary and discussions.

\subsubsection{Environment For Channel Modeling}

Among air-to-ground path loss models, [28] gained popularity due to the consideration of urban environments. In particular, high-rise dense urban environments with buildings of $60 \mathrm{~m}$ height on average is represented by adjusting the probability of having LoS links in [28]. However, especially cores of modern metropolitans often consist of even higherrise buildings, with an average height of buildings of $100 \mathrm{~m}$. Urban environments can be classified based on the average heights of the buildings more precisely as follows [148]:

1. High-rise Urban: Consists of buildings with several floors $(\leq 30 \mathrm{~m})$.

2. Very High-rise Urban: Consists of densely located buildings with several tens of floors (30 to $100 \mathrm{~m}$ ).

3. Skyscraper Urban: Consists of densely located skyscrapers ( $\geq 100 \mathrm{~m})$.

Note that we propose the last case to describe communications with drones in cores of metropolitans such as Hong Kong (553 skyscrapers), New York (799 skyscrapers), and Tokyo (281 skyscrapers) [149]. The particulars of skyscraper urban environment are the extreme heights of the buildings, population density, and deep street canyons, which makes it a category of its own with unique propagation characteristics.

In such environments, the altitude of the drone-BSs with respect to building heights can have critical effects. In the literature, drone-BSs are often termed as low-altitude platforms (LAPs), which are platforms lower than $10 \mathrm{~km}$ in [28]. Because the operational altitude can vary significantly from tens of meters to kilometres, we would like to describe the drone-BSs lower than $100 \mathrm{~m}$ as ultra-low-altitude platforms (ULAPs), where $100 \mathrm{~m}$ represents the near roof-top level of many skyscrapers. Besides the path loss model, selection of altitude has various effects from the type of drone, to legal permission required, to endurance [2]. For instance, in modern metropolitans, the density of the population may not 
require serving a larger area, but providing a very good service quality to certain users [52]. In that case, using ULAPs at lower altitudes can be an efficient option.

In this study, we identify the skyscraper urban environment as a scenario where a widely used channel model [28] may be too simple. Since there is no widely accepted channel model for ULAPs, following a similar strategy as [28], we adopt a channel model from [148] to be used with city maps in this environment. The model in [28] is probabilistic in the sense that the probability of a link being $(\mathrm{N}) \operatorname{LoS}$ depends on the distance between the user and the drone-BS. On the other hand, the proposed model is deterministic, which means that it is known whether a link is LoS or NLoS.

The purpose of this study is not so much to propose a particular model for the air-toground channel. Indeed, many models could be fitted to the data points of various simulation setups. It is rather to propose a particular type of model, that is, one that uses basic information about building shapes and city layout, along with blockage, reflection, and around-the-corner-diffraction propagation mechanisms. Indeed, these considerations are necessary to reasonably model the channel in this case.

\subsubsection{Channel Models for Low and Ultra-low Altitude Platforms}

One of the main methods to determine whether an air-to-ground link is LoS or NLoS is using probabilistic models based on the averages of the characteristics of the environment (e.g., average height and number of buildings per $\mathrm{km}^{2}$ etc.), and the location of the droneBS relative to the user [28].

Probabilistic Model: Recently, a probabilistic model (PM) for air-to-ground path loss is presented in [28], and a similar model for terrestrial links can be found in [150]. The PM depends on combining the free space path loss with excessive loss due to environmental obstacles. Free-space loss is modelled via the Friis equation, which is given as follows for a user located at $(x, y)$ and the drone-BS at $\left(x_{D}, y_{D}, h\right)$ :

$$
L_{f}(h, r)=20 \log \left(4 \pi f_{c} / c\right)+20 \log (d)[\mathrm{dB}],
$$

where $d=\sqrt{h^{2}+r^{2}}$ is the distance between a user and drone-BS in meters, $h$ is the altitude of the drone-BS, and $r=\sqrt{\left(x_{D}-x\right)^{2}+\left(y_{D}-y\right)^{2}}$ is the horizontal distance between the 
drone-BS and the user. The diffraction and reflection due to environmental obstacles cause excessive loss $(\eta)$, which is less for a LoS link $\left(\eta_{L o S}\right)$ compared to a NLoS link $\left(\eta_{N L O S}\right)$. The following probability of LoS is used to combine excessive loss with free-space loss:

$$
P(h, r)=(1+a \exp (-b(\arctan (h / r)-a)))^{-1},
$$

where $a$ and $b$ are constant values depending on the environment. Then, the excessive loss can be written as

$$
L_{e}(h, r)=P(h, r) \eta_{\mathrm{LoS}}+(1-P(h, r)) \eta_{\mathrm{NLoS}}[\mathrm{dB}] .
$$

Hence, total loss becomes $L_{f}(h, r)+L_{e}(h, r)$. Although PM is compact and useful, the imprecise determination of the quality of links can lead to inefficient drone-BS placements. That can be a critical problem for two reasons: First, especially ULAPs are energy-critical UAVs, and inefficient 3D placement can lead to signal transmission with excessive power to ensure service quality. Second, if the drone-BSs are not exploited properly, drone-BSs may not provide any profit to the operator (except critical situations). To combat this problem, more precision can be obtained by making use of information on environment, which can be easily gathered from city maps.

Urban Canyon Model: Note that city maps can be used to determine a LoS path, and the remaining NLoS areas, also providing computational efficiency by eliminating the need to calculate $P(h, r)$, which is based on a deterministic approach. The urban canyon model (UCM) investigated in this section depends on a recently released below roof-top path loss model by ITU-R, which requires the knowledge on the locations of the BSs and the users, as well as the street widths, shapes and alignments [148]. This model can also be valid for drone-BSs when ignoring near-field effects of flight and machinery, such as attenuation due to drone's body and effects of turbulence, similar to the approach of the previously presented air-to-ground models [25,28,151]. In [148], two-ray plane earth reflection model is utilized for LoS links. In this case, the median of the LoS path loss is given in [148, p.8] as

$$
L_{L o S}=L_{b}+6+K \log \left(d / R_{b}\right) \quad[\mathrm{dB}] .
$$

In the above, $R_{b} \approx \frac{4 h_{D} h_{u}}{\lambda}$ is the break-point distance, $h_{D}$ and $h_{u}$ are the heights of the drone and the user, respectively. If $d \leq R_{b}, K=20$, and $K=40$ otherwise. For NLoS links, path loss depends on the distance of the drone-BS and the user from the intersections of LoS 

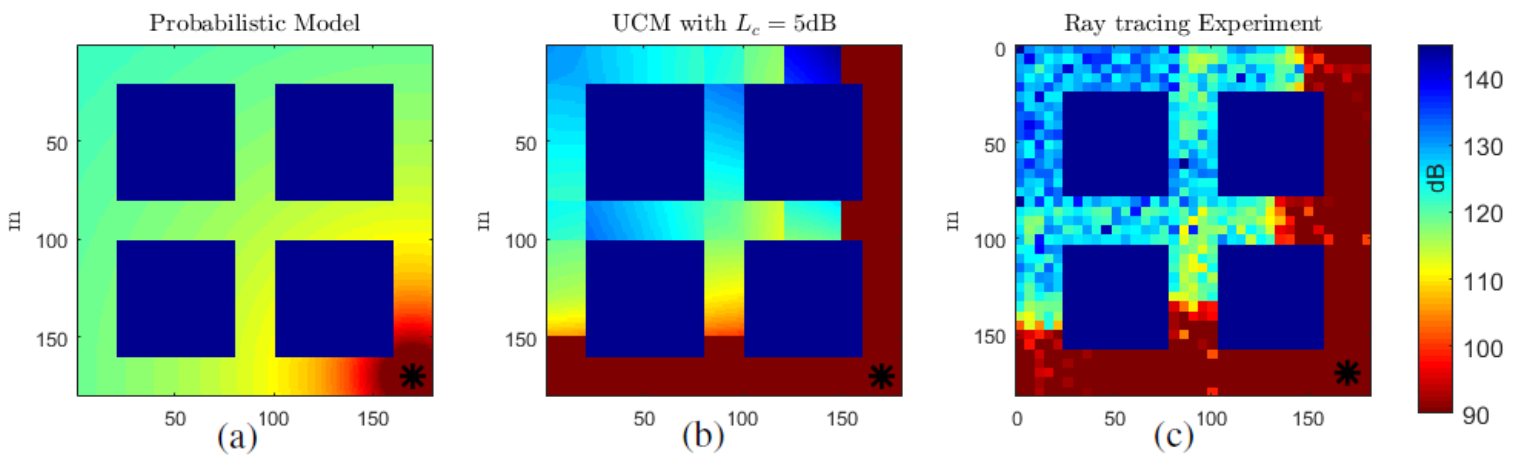

Figure 2.9: Path loss patterns for a drone-BS at $50 \mathrm{~m}$ altitude.
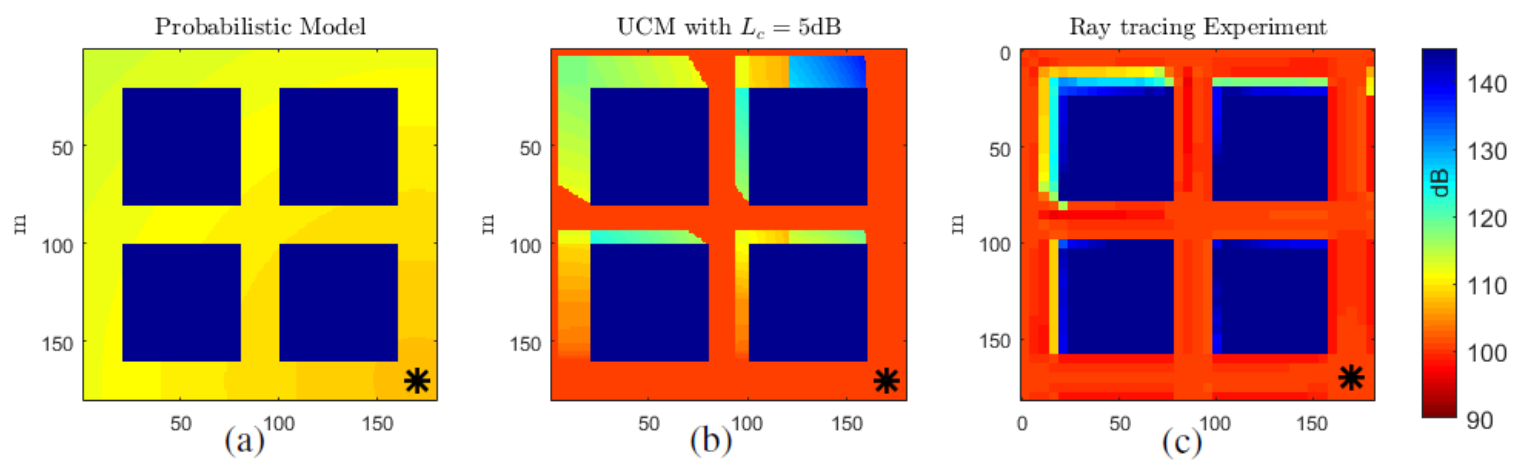

Figure 2.10: Path loss patterns for a drone-BS at $1000 \mathrm{~m}$ altitude.

and NLoS streets, i.e, corners [148, Chap. 4.1.2]. Let $C$ in meters represent effective corner region (CR) from LoS street into NLoS street. A user is assumed to be in CR if distance of the user in NLoS street, $r_{c}^{u}$, satisfies $\frac{w_{d}}{2}+1 \leq r_{c}^{u} \leq \frac{w_{d}}{2}+1+d_{c}$. For this case (in dB),

$$
L_{c r}=\left\{\begin{array}{l}
\frac{L_{c}}{\log \left(1+d_{c}\right)} \log \left(l_{u, c}-\frac{w_{d}}{2}\right), \text { if } \frac{w_{d}}{2}+1 \leq l_{u, c} \leq \frac{w_{d}}{2}+1+d_{c} \\
L_{c}, \text { if } l_{u, c}>0.5 w_{d}+1+d_{c}
\end{array}\right.
$$

where $l_{u, c}$ represents the user's distance from the corner, $w_{d}$ is the width of the streets as shown in [148, Fig. 3]. Corner loss region, and corner loss are represented by $d_{c}$ and $L_{c}$, respectively. While (2.32) represents strong refraction around corners, (2.33) is for the increased loss when proceeded into the street [148, Fig. 4]. Attenuation beyond the corner region is (in $\mathrm{dB})$

$$
L_{a t t}=60\left(\log _{10}\left(l_{u, c}+l_{d, c}\right)-\log _{10}\left(l_{u, c}+0.5 w_{d}+d_{c}\right)\right) .
$$

Note that $l_{d, c}$ denotes the distance of drone-BS to the corner region as shown in [148, Fig. 
3]. Finally, the path loss for a NLoS link becomes, $L_{N L o S}=L_{L o S}+L_{c r}+L_{a t t}$. Also, UCM is reciprocal under proper assumptions.

Note that $L_{c}$ is a critical parameter in determining the path loss, as it determines the average amount of diffraction, and given as $20 \mathrm{~dB}$ in [148]. We propose to adjust $L_{c}$ based on the altitude with the objective of minimizing root-mean-squared error (RMSE) by grid search. We see that $L_{c}$ decreases with increasing altitude, and the opposite is true for decreasing altitude, due to change in the amount of diffraction. For instance, if the altitude of a LAP is strictly higher than the roof-top level of the buildings, there will be more diffraction. For ULAPs at altitudes strictly lower than roof-top levels of the buildings, diffraction is not likely to occur, and $L_{c}$ must be higher than the one in the previous case, which is verified by our experiments in the following section. For instance, $L_{c}$ is determined for certain altitudes in this study, however, DMS can update $L_{c}$ values based on the current altitude of the drone-BS. In that sense, the proposed UCM can be useful as an easily adjustable path loss model with one single parameter.

\subsubsection{Simulation Results}

The channel models presented in the preceding sections, PM and UCM, are evaluated based on the ray-tracing simulations on a toy map of a Manhattan-grid skyscraper environment with the parameters provided in Table 2.5. The path loss amount is presented as a heat map in Figs. 2.9 and 2.10, where $h$ is $50 \mathrm{~m}$ and $1000 \mathrm{~m}$, respectively. The drone-BS is hovering on the point marked with a black asterisk, which is the center of the intersection at $(170,170, h)$. The ray-tracing simulations are conducted via Wireless Insite ${ }^{1}$ with $5 \times 5 \mathrm{~m}$ pixels. A toy map with fixed building height is preferred to clearly demonstrate the effect of altitude and capability of channel models. Note that varying the height of the buildings does not contribute much to this discussion: If the drone-BS hovers at $50 \mathrm{~m}$, it is lower than majority of the buildings in a skyscraper environment (NLoS except the streets where the drone-BS is located), and at $1000 \mathrm{~m}$, it is significantly higher (mostly LoS). The $200 \mathrm{~m} \mathrm{x}$ $200 \mathrm{~m}$ subsection of the area in Figs. 2.9 and 2.10 is sufficient to show main characteristics of PM and UCM, as the map is symmetric. Note that the actual map area in Table 2.5 is the repetition of the $2 \times 2$ building structure.

\footnotetext{
${ }^{1}$ http://www.remcom.com/wireless-insite
} 


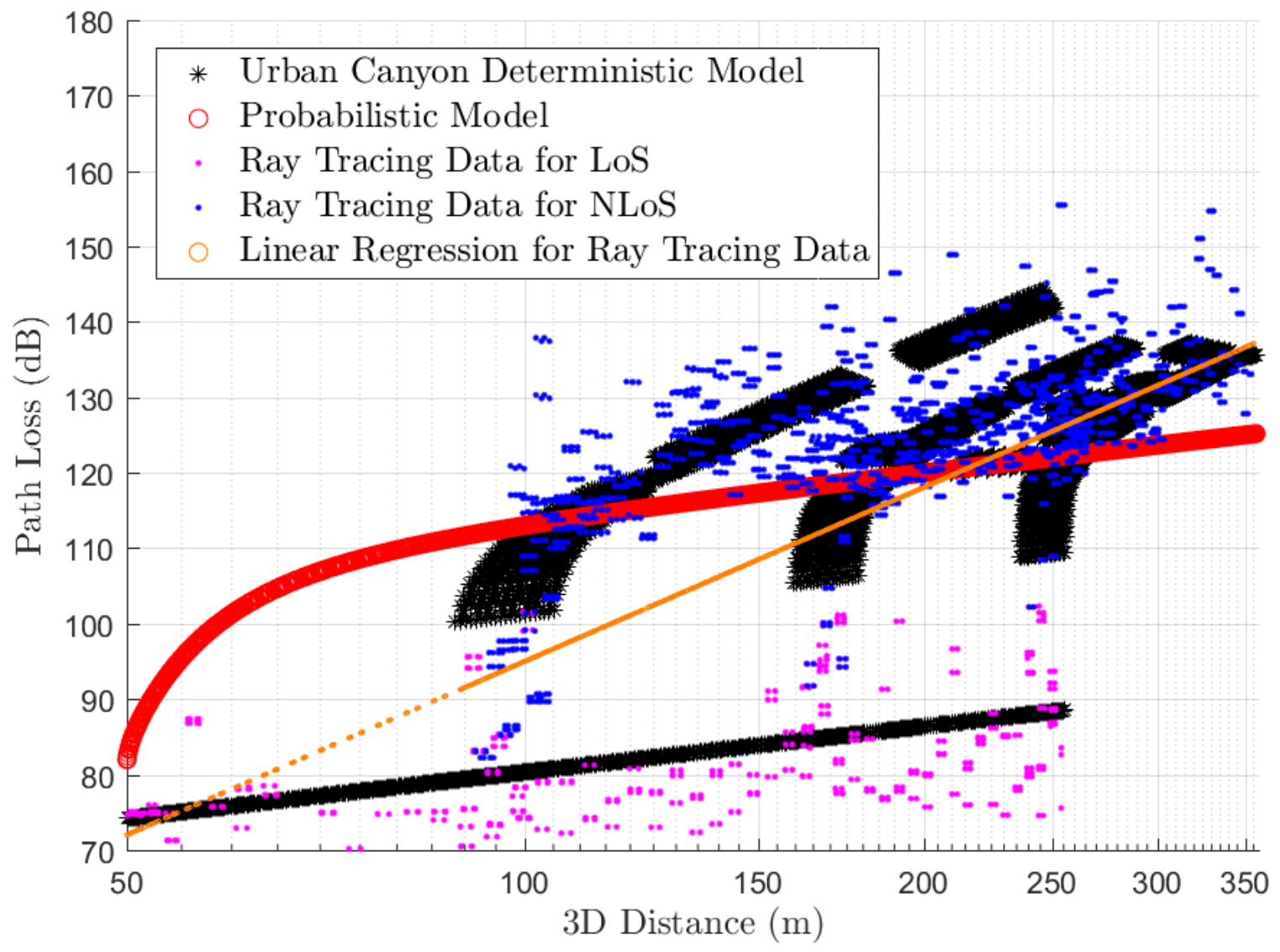

Figure 2.11: Propagation data at $50 \mathrm{~m}$ in comparison to ray-tracing simulations. 


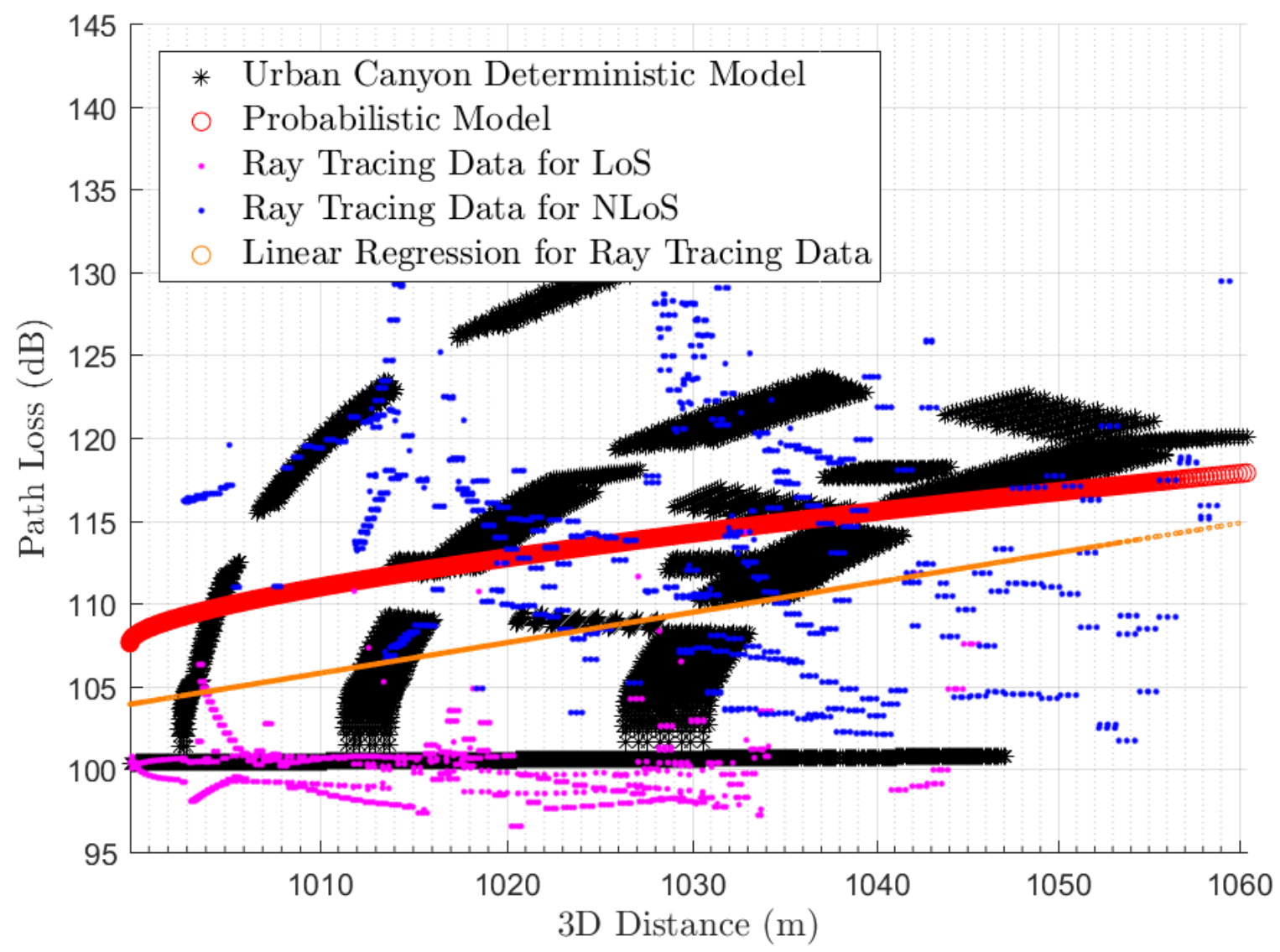

Figure 2.12: Propagation data at $1000 \mathrm{~m}$ in comparison to ray-tracing simulations. 
Table 2.5: Parameters for ray-tracing and channel models.

\begin{tabular}{|l|l|}
\hline Parameter & Value \\
\hline Conductivity of walls & $150 \times 10^{-4} \mathrm{~S} / \mathrm{m}$ \\
\hline Conductivity of ground & $5 \times 10^{-4} \mathrm{~S} / \mathrm{m}$ \\
\hline Permittivity of walls & 15 \\
\hline Permittivity of ground & 5.72 \\
\hline Thickness (walls and ground) & $0.3 \mathrm{~m}$ \\
\hline Building height $\left(h_{B}\right)$ & $100 \mathrm{~m}$ \\
\hline Building width & $60 \mathrm{~m}$ \\
\hline Street width $\left(w_{d}\right)$ & $20 \mathrm{~m}$ \\
\hline Map area & $500 \mathrm{~m} \mathrm{x} \mathrm{500} \mathrm{m}$ \\
\hline Carrier frequency $\left(f_{c}\right)$ & $2.5 \mathrm{GHz}$ \\
\hline Receiver height $\left(h_{u}\right)$ & $1 \mathrm{~m}$ \\
\hline Altitude (h) & $h \in\{50 \mathrm{~m}, 1000 \mathrm{~m}\}$ \\
\hline$L_{c}$ (urban) & $L_{c} \in\{30 \mathrm{~dB}, 5 \mathrm{~dB}\}$ \\
\hline$d_{c}$ (urban) & $30 \mathrm{~m}[148]$ \\
\hline$\eta_{L o S}$ (high-rise) & $2.3 \mathrm{~dB}[28]$ \\
\hline$\eta_{N L o S}$ (high-rise) & $34 \mathrm{~dB}[28]$ \\
\hline
\end{tabular}

According to PM, the probability of having a LoS connection only depends on distance and altitude as given in (2.29). Therefore, the PM cannot capture LoS on the streets, as seen in Figs. 2.9a and 2.10a. On the other hand, PM can be suitable for altitudes much higher than the buildings, as in the case of Fig. 2.10. In that case, the propagation is dominated by free space loss, as $h$ becomes a much larger quantity than $r$. In other words, PM is dominated by $L_{f}(h, r)$ as $L_{e}(h, r)$ approaches $\eta_{L o S}$, and LoS probability approaches 1 for all ground locations. This situation can also be observed in Fig. 2.11 and Fig. 2.12, where the resemblance of PM to the linear regression line of the ray-tracing data is higher for $1000 \mathrm{~m}$ compared to $50 \mathrm{~m}$.

There is a significant improvement in following the propagation patterns of ray-tracing simulations via UCM as observed from Figs. 2.9b and 2.10b. Fig. 2.11 and Fig. 2.12 shows that UCM provides a curve for each street, which coincides with ray-tracing values. 
Table 2.6: RMSE of propagation models for several building and drone-BS heights, $h_{B}$ and $h$, respectively.

\begin{tabular}{|c|c|c|c|c|c|c|c|}
\hline Model RMSE & \multicolumn{3}{|c|}{$\operatorname{LoS}(\mathrm{dB})$} & \multicolumn{2}{|c|}{ NLoS (dB) } & \multicolumn{2}{|c|}{ Overall (dB) } \\
\hline \multirow{4}{*}{ Probabilistic } & $\mathbf{h}_{B} h$ & $50 \mathrm{~m}$ & $1000 \mathrm{~m}$ & $50 \mathrm{~m}$ & $1000 \mathrm{~m}$ & $50 \mathrm{~m}$ & $1000 \mathrm{~m}$ \\
\hline & $60 \mathrm{~m}$ & 34.27 & 11.20 & 10.15 & 10.41 & 16.97 & 10.31 \\
\hline & $100 \mathrm{~m}$ & 34.24 & 11.97 & 10.33 & 8.85 & 17.04 & 9.73 \\
\hline & Random & 34.30 & 11.40 & 10.86 & 10.91 & 17.30 & 10.43 \\
\hline \multirow{4}{*}{ Urban Canyon } & $h_{B} h$ & $50 \mathrm{~m}$ & $1000 \mathrm{~m}$ & $50 \mathrm{~m}$ & $1000 \mathrm{~m}$ & $50 \mathrm{~m}$ & $1000 \mathrm{~m}$ \\
\hline & $60 \mathrm{~m}$ & 7.12 & 2.24 & 9.04 & 13.56 & 8.40 & 6.53 \\
\hline & $100 \mathrm{~m}$ & 7.11 & 1.95 & 8.82 & 12.49 & 8.19 & 9.07 \\
\hline & Random & 7.21 & 1.44 & 9.11 & 13.66 & 8.47 & 9.71 \\
\hline
\end{tabular}

Especially, since the proposed UCM can capture no-diffraction $(h=50 \mathrm{~m})$ and strongdiffraction ( $h=1000 \mathrm{~m}$ ) cases by adjusting $L_{c}$ in (2.32) and (2.33), it provides better overall RMSE values compared to PM as shown in Table 2.6. For LoS points, environmental awareness provided by UCM leads to more than $25 \mathrm{~dB}$ less RMSE compared to PM, which cannot capture LoS links of the points on the same streets (horizontal and vertical) with drone-BS hovering at $50 \mathrm{~m}$. When the drone-BS hovers at $1000 \mathrm{~m}$, RMSE for LoS links is approximately $10 \mathrm{~dB}$ better with UCM compared to PM. However, for NLoS links, both path loss models perform similarly, and UCM is slightly worse at $1000 \mathrm{~m}$, which is due to adjusting only $L_{c}$.

Note that the UCM can be used for many urban environments. To demonstrate the capabilities of the UCM, another set of simulations are conducted with uniformly-distributed random heights, $h_{B} \in[60,150] \mathrm{m}$, without changing the parameters of the UCM corresponding to below and above roof-top cases. The similarity of the RMSE values show that UCM can be applicable in different environments with similar urban characteristics.

In order to provide further insights, 50 users are randomly distributed in the area. The number of covered users, $\beta$, is chosen as the performance metric, which can easily be used to represent sum rate, revenue, or other key performance indicators. A user is assumed to be covered based on a maximum tolerable path loss threshold, $\gamma$, such that a user is 


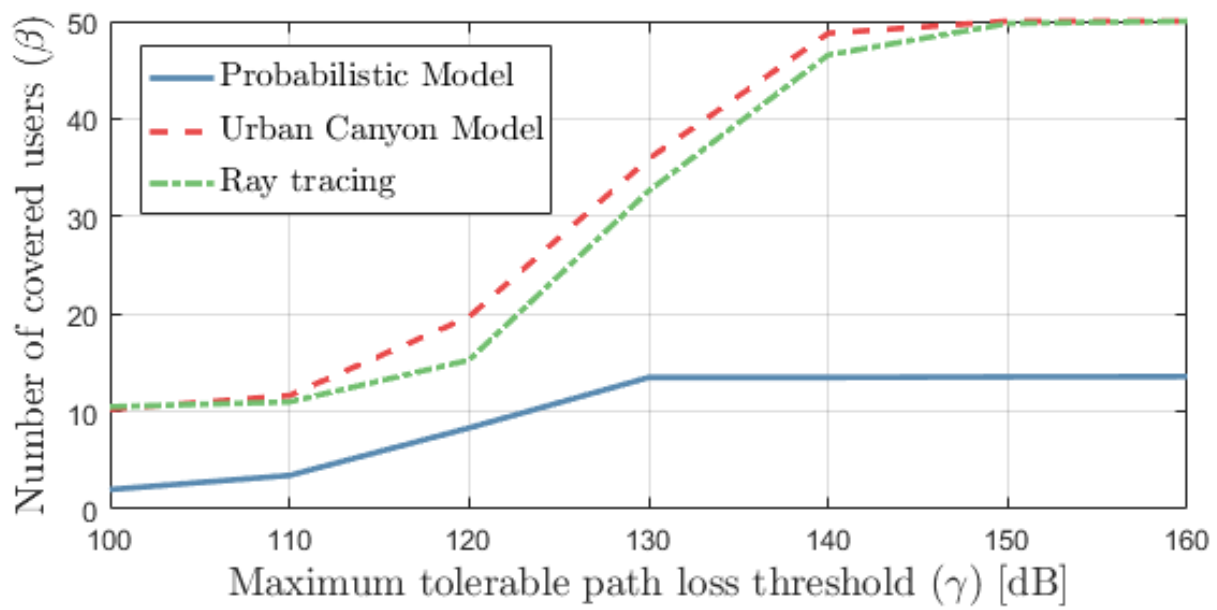

Figure 2.13: Number of covered users with PM, UCM, and ray-tracing simulations.

covered if the path loss is less than $\gamma$ at the user's location, and uncovered otherwise. Maximum number of users that can be covered via PM is obtained by the efficient 3-D placement method proposed in [31] with the additional constraint of $h \leq 100 \mathrm{~m}$ to enforce the scenario in Fig. 2.9. High rise urban environment parameters are used for PM, and other simulation parameters can be found in [31, Table I, II]. While the drone-BS is placed at the best location by the algorithm in [31], it is fixed at the center of the environment for ray-tracing and UCM at $(252.5252 .5,50)$.

Fig. 2.13 shows $\beta$ vs $\gamma$ averaged over 100 Monte Carlo runs. Results show that UCM and ray-tracing have similar coverage, which increase with increasing $\gamma$, due to capturing street propagation. However, PM cannot increase coverage after $\gamma=130 \mathrm{~dB}$, due to limitation on the altitude, as in (2.29). Note that PM dramatically underestimates the number of covered users, whereas UCM is very close to ray-tracing simulations.

\subsection{Summary and Discussions}

Inaccurate path loss estimation resulting from probabilistic models may cause several problems for the drone-BSs framework in both the planning and operation phases. For instance, network operators may need to determine the number of drone-BSs to be dispatched, their flight duration, coverage area, and potential revenue. In particular, we observed that a 
widely used PM may not be sufficient to accurately reflect the propagation scenario, and indeed may wrongly estimate the channel path loss by over $34 \mathrm{~dB}$. Consequently, the number of users covered with a drone-BS, and number of required drone-BSs cannot be determined accurately. On the other hand, accuracy in estimating the path loss with deterministic models can enable environment-aware placement methods, and hence, more precise planning for drone-BS operations.

The PM is still useful when the environment information is not available, or it is computationally costly to derive such information. In this case, the precision loss due to lack of information can be somewhat alleviated by using PM as precisely as possible by considering user locations when placing the drone-BS. Therefore, we discussed an efficient 3-D placement method such that the coverage area fits the users in it. In other words, no coverage area is wasted/empty for a snapshot of the system. Furthermore, we extended the proposed 3-D placement method to serve mmWave-drone-BSs by considering the blockage effect of users on each other. We showed the effect of the density of the human blockers on the coverage performance of a mmWave-drone-BS when efficient 3-D placement is used.

The complex nature of drone-BS operations may require a drone-BS management framework, which is capable of collecting and analysing large amounts of data for operational purposes. Hence, preferring deterministic methods can be acceptable in the presence of such mechanisms. Moreover, a DMF can be useful for integrating drone-BSs with appropriate radio access technologies (RATs) to the existing terrestrial network [2]. For instance, mmWave-drone-BSs can be preferred over the RF drone-BSs, since the latter ones can cause severe interference. Furthermore, it may be possible to influence user behaviour to change the user density or alter the blockage. In order to do so, incentives may be offered to users. Therefore, in the following chapters, we user-centric spatial network configuration and the integration of drone-BSs into the existing wireless networks from a wide perspective. 


\section{Chapter 3}

\section{User-Centric Spatial Network Configuration}

In this section, we investigate the synergy between the deployment of drone-BSs and designing the incentives for user-in-the-loop (UIL) schemes to improve efficiency and increase profitability of wireless networks. Cell association, traffic management, and load balancing can be thought of as related research areas. The difference between the previous schemes and this one is the following: Traditionally, the base station (BS) is fixed and the locations of users are random. In our case, the base station is mobile with a varying coverage area as described in the previous chapter, and mobility of the users is influence-able towards the benefit of the network. Hence, spatial network configuration (SNC) can be performed.

Efficiently combining drone-BSs and user involvement models in cellular networks with the objective of maximizing profit while satisfying QoS requirements is a rather involved design problem. Both drone-BSs and user involvement models have their unique challenges [2,152-154]. To the best of our knowledge, this study presents the first system that considers both the mobility of the users and drone-BSs. The contributions of the study presented in this chapter can be summarized as follows:

- The gain from UIL, which is a user involvement model tailored for wireless networks (Section 3.1), for a uniform user distribution is analysed in Section 3.5. Our analysis show that $50 \%$ relative gain can be obtained by incorporating UIL. Besides providing insights on the profitability of UIL, the analysis can be useful in cases of insufficient or incorrect information on user locations, and/or very high user mobility. 
- When information on user location is available, the optimal incentive for an uncovered user at a given ground distance from the coverage of area of a base station is obtained in Section 3.2.1.

- A framework for SNC, consisting of 3-D placement of a drone-BS, and incentive design for UIL, is presented. In Section 3.3, an uncoordinated-SNC method is proposed to utilize the framework. It is shown that for a user, the gain from the UIL is strictly affected by user's proximity to the location of the drone-BS.

- In order to improve system performance by performing 3-D placement with consideration to incentive design for UIL, a joint-SNC (JSNC) problem is formulated in Section 3.6. The proposed JSNC problem is efficiently solvable via interior-point optimization, however, the computational complexity is very high.

- A reduced-complexity joint-SNC problem, semi-JSNC, is formulated in Section 3.7. Simulation results show that semi-JSNC can be more than 10 times faster than JSNC, while providing similar gain.

- All of the proposed SNC methods with varying computational complexities and accuracies, are shown to increase the profit of the network operator, as well as the number of served users by the drone-BS without increasing transmission power.

\subsection{User Involvement Models}

User involvement is utilized as a method to improve system performance, with respect to user's perception or via user cooperation, for the purpose of application adaption, accurate crowd-sensing, improved cybersecurity and so on [153-179,179-189]. Nunes et al. provide a survey in [153] where they argue that cyber-physical systems can benefit significantly from considering human element as a part of the system, instead of treating it as an uncontrollable external component. In fact, once the target benefits of networks are determined, main components of many systems with user involvement can be summarized as follows.

- Role of the user: The cooperative role of the user is the first building block of systems with user involvement. In general, the user may take actions, be passive, or systems 
with hybrid user roles are also possible [153].

- Incentive design: According to the role of the user, incentives are designed. A good incentive design should be persuasive enough to convince users to cooperate with the network operator ${ }^{1}$, and at the same time should maximize network's target benefits (e.g., green and profitable wireless network provisioning).

Target benefit of the network determines the role and tasks of the users to be involved. Different roles can result in different system designs. Users can have a passive role, e.g., in many crowd-sensing applications such as [159-162] users only provide their data. For instance, incentives are offered to users to improve crowd-sensing data quality in [159]. In [186], user perception is utilized to fine-tune QoS, which in turn provides energy savings. The study in [155] is an example of a hybrid system, where users can have both passive and active roles. For instance, in [155], users either only watch or choose to share advertisements to obtain incentives. The target benefit in this study is to turn users into agents to distribute advertisement content, while developing sponsored data plans to create a win-win situation. Alternatively, users may be more actively involved by taking an action affecting the system performance, e.g., in case of UIL, the user may move to a position with better SINR [171], or in [156-158] users share their resources with other users to support network operator's services. In [156], licensed users trade under utilized spectrum resources with unlicensed users to improve spectrum utilization efficiency. In [157] and [158], users provide access points to improve connectivity of wireless networks. When the UIL method is applied in [171,172,185,190,191], users receive monetary or non-monetary incentives (e.g., discount on service fees, improved service quality, reducing environmental impact of communications) so that the network operation can become more efficient, e.g., in terms of energy expenditure, wireless resource usage etc., and increase number of served users via demand shaping [171]. For instance, users can receive incentives to delay their demand, or move to a better position where providing wireless services is more efficient. Hence, the users' demand can be shaped spatiotemporally. Note that the users can choose to comply with the offers or not, and making persuasive and profitable offers are key for the success of UIL systems.

\footnotetext{
${ }^{1}$ The "network operator" is used here in a broader sense, such that it may be an actual network operator, e.g., AT\&T, Turkcell, Bell, or a person operating an application using the existing network, e.g., Foursquare, WhatsApp.
} 
Incentive design has been puzzling researchers as a multi-disciplinary issue with complex factors. Many incentive methods have been developed, where they can be broadly categorized as follows [154].

- Non-monetary incentives: When the network operator provides incentives that are costless to the operator, the persuasiveness of the incentive depends on the willingness of the users, or gaming [160].

- Monetary incentives: When costly incentives are offered to the users, there is a tradeoff between the persuasiveness of the offer and profitability of the operation. Moreover, even with monetary incentives, not all the users will comply [192].

The studies on incentive methods either rely on hypothetical assumptions (e.g., [159, 164, 193, 194]), or field studies (e.g., [168, 169, 192, 195]). Both methods suffer from reliability, as the statistical assumptions are hard to justify and many field studies are limited to a small group of participants with similar demographics, e.g., university students. Moreover, it is hard to generalize the results of a field study for all applications, since the user tasks and roles may differ. Nevertheless, in [168] effectiveness of monetary examples are investigated based on an existing field study that has cumbersome requirements for the participants. This study with 36 participants suggests that if variable amounts of incentives are offered for similar tasks, the user persuasion rate to complete the task can be slightly higher than offering a uniform amount or a hidden amount that is revealed after completion [168]. A similar study is conducted in [169] with the purpose of crowd-sensing, i.e., users share their desired data in exchange with some amount of incentive. Similar to the study in [168], offering variable incentives resulted more and higher-quality data collection from a total of 55 participants. Note that the amount of incentives offered in these studies does not depend on the system requirements, rather they are static. On the other hand, studies such as [187-189] consider dynamic factors to determine incentives, such as availability of resources, demand towards users' data, behavior of other users and so on. In [192], a unique survey is conducted with 100 participants (twice the amount of participants in previous studies) to understand the behavior of wireless network users in cooperating with the network operator. Certainly, the study in [192] is inadequate to draw substantial conclusions on this complex issue, however, it is at least as comprehensive as the previous studies, and targeted towards operating wireless networks, rather than another field or purpose. Therefore, instead of 
using hypothetical assumptions about the user behavior, the results in [192] is used for the analysis in this study.

In particular, UIL is an emerging user involvement method, which introduces the user behaviour as another system block of wireless communication networks. The interesting phenomenon here is that the element of user behaviour is neither completely under control, such as transmitter or receiver blocks, nor completely out of control, such as noise. The objective of UIL is to configure the network in space and time by creating win-win situations for both the users and the network operators. There are two main UIL schemes: Spatial-UIL, which is based on shaping the demand spatially, and temporal-UIL, which is based on delaying the demand and shaping it in time domain. There have been several studies investigating performance and effects of these UIL schemes. The first survey results on UIL are presented in [192], where tendency of the users to accept offers of the network operator based on the service in demand, amount of spatial or temporal displacement, and incentive type and amount is investigated. In [196], UIL is utilized for a novel load balancing approach. Similarly, an advanced spatial-UIL scheme to mitigate backhaul congestion for HetHetNets is proposed in [190]. A comprehensive discussion on UIL is provided in [152], where motivations, various UIL schemes, survey results, and sustainability of wireless networks with UIL from economic and green networking perspectives are investigated. Finally, in [197], user-behaviour-awareness is introduced to increase efficiency of D2D communications in terms of energy savings, increased reliability, and guaranteed quality-of-experience. For this purpose, a user-behavior-aware Stackelberg repeated game (UBeAS) is developed to solve a resource allocation problem in distributed D2D communications by utilizing the UIL framework to reflect D2D user-behaviour.

\subsection{System Model for Evaluations}

We consider a scenario in which the existing infrastructure of mobile network operators is temporarily insufficient to respond to the demand in a finite region, $\mathcal{W}$, containing a set of users, $\mathbb{U}$. We assume that the location of each user in 2-D horizontal space, $\left(x_{i}, y_{i}\right) \forall i \in$ $\mathbb{U}$, and quality of service (QoS) requirement of the users in terms of maximum tolerable path loss, $\gamma$, are known. A drone-BS is to be utilized to support the network as shown in 


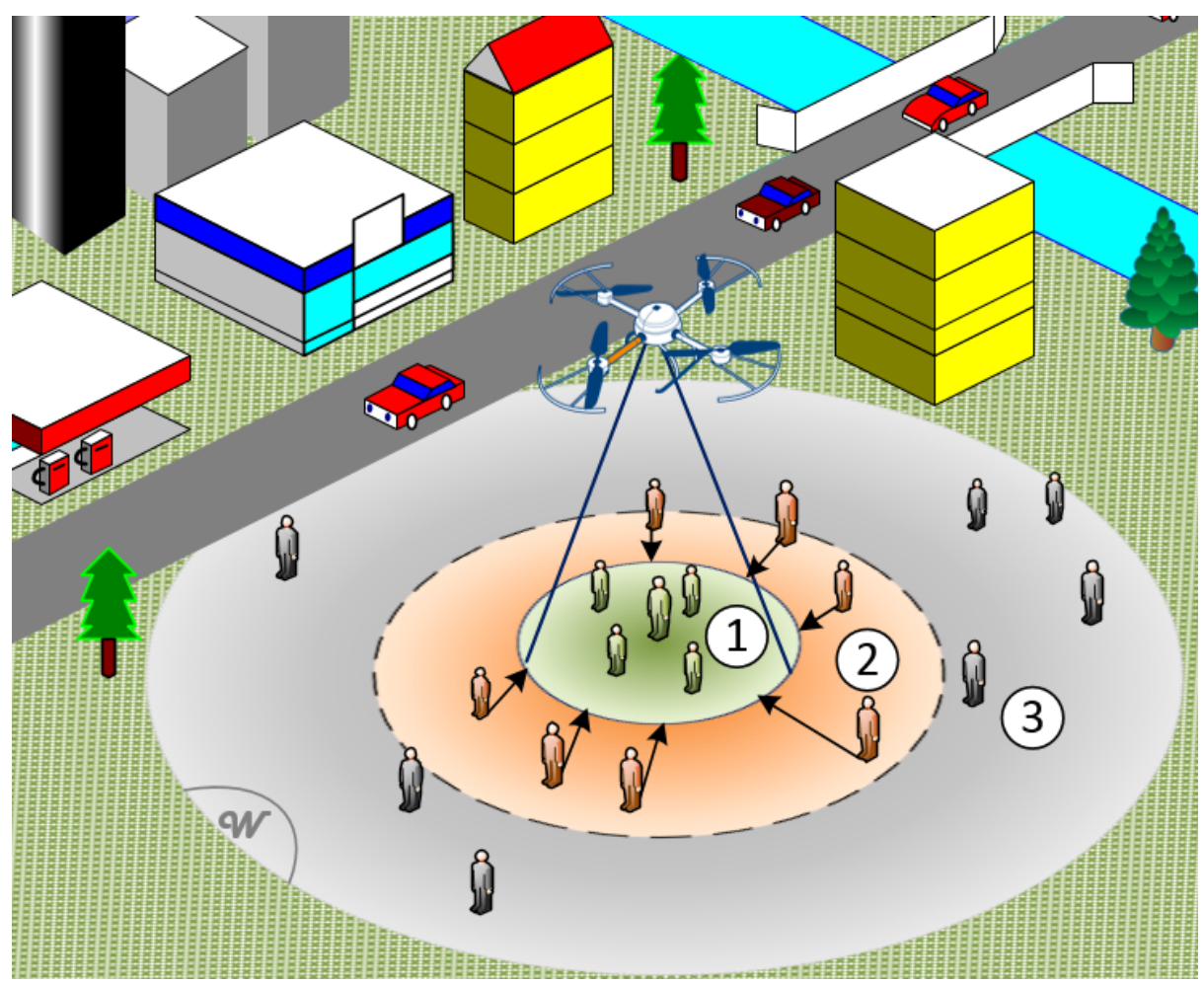

Figure 3.1: Description of SNC: Users in region (1) are covered without additional cost. Users in region (2) are within the extended coverage region, therefore, they receive an incentive offer to move towards the coverage area. Users in region (3) cannot be covered even with UIL, and hence do not receive an offer.

Fig. 3.1 by offloading as many users as possible from the network, while satisfying the QoS requirements and maintaining profitability of the network. Air-to-ground channel model is as in Section 2.1.1. Utilized spatial UIL model is explained next.

\subsubsection{User-in-the-loop Model}

UIL is a closed loop model with the user as the system to influence for increasing the efficiency of wireless networks. UIL is based on offering incentives to users to persuade them to take specific actions, such as delaying their demand in time, or changing their location in space. These incentives can be discounts on the service price, higher data rates, reducing carbon footprint (green networking), or even penalties for refusing the change [152]. Therefore, although at a cost, potential wastes of energy and capacity can be prevented, resulting a more efficient network. 
UIL can be used either spatially, where the user moves in space to obtain better links with the destination (higher spectral efficiency), or temporarily, in which case the user delays the demand in time. Assuming a drone-BS is opportunistically utilized for a specific time period, and the objective is to offload as many users as possible to the drone-BS during its utilization, a spatial UIL scheme is considered in this study.

In order to investigate the reaction of the users to various UIL schemes for data, video and voice services, a detailed survey is conducted in [192]. For data services, the probability of a user $i$ accepting the incentive, $\tau_{i}$, which is a discount on the cost of the service for user $i$, and moving a distance less than or equal to $d_{i}=\sqrt{\Delta x_{i}^{2}+\Delta y_{i}^{2}}$ is given as

$$
\text { P }\left\{\text { user } i \text { moves distance } \leq d_{i}\right\}=\mathrm{e}^{-\beta\left(\tau_{i}\right) d_{i}} \text {, }
$$

where $\beta\left(\tau_{i}\right)$ is the persuasion parameter.

In [192], $\beta(\tau)$ is calculated based on four different incentives, namely, $\tau=0.2,0.4,0.6$, and 0.8 , corresponding to discount amounts of $20 \%, 40 \%, 60 \%$, and $80 \%$, respectively. These incentive amounts provide the persuasion values, $\beta(\tau)=0.0244,0.0164,0.0117$, and 0.0082 , respectively. A continuous and tractable function for $\beta(\tau)$ is obtained by logarithmic curve fitting in our study, such that

$$
\beta(\tau)=k_{1} \ln (\tau)+k_{2}
$$

where $k_{1}=-0.01166$ and $k_{2}=0.005676$ for $\tau \in[0,1]$, where 0 and 1 corresponds to "no incentive" and "free service" (100\% off), respectively. In Fig. 3.2, the probability of being persuaded in (3.1) is shown both with the points from the survey in [192], and the fitting in (3.2). It is observed that (3.2) is a proper fit for the provided values.

Proper selection of UIL system parameters, such as $\tau_{i}$ and $d_{i}$, has a key role in the performance of any UIL scheme [152, 190, 192, 196, 197]. The incentivized user movement suggestion may be a function of a variety of elements, including user type, application type and urgency, the need for UIL, and so on. It may provide less ossified extension regions, on the contrary to Fig. 5.1. For instance, if there is a compliant user e.g., a student type user, the user may be given an incentive even when located in region 3. On the other hand, a non-compliant user, e.g., a business type user, may not receive incentives even if the user is located in region 2. Note that, compliance level of a user may change if the application is 


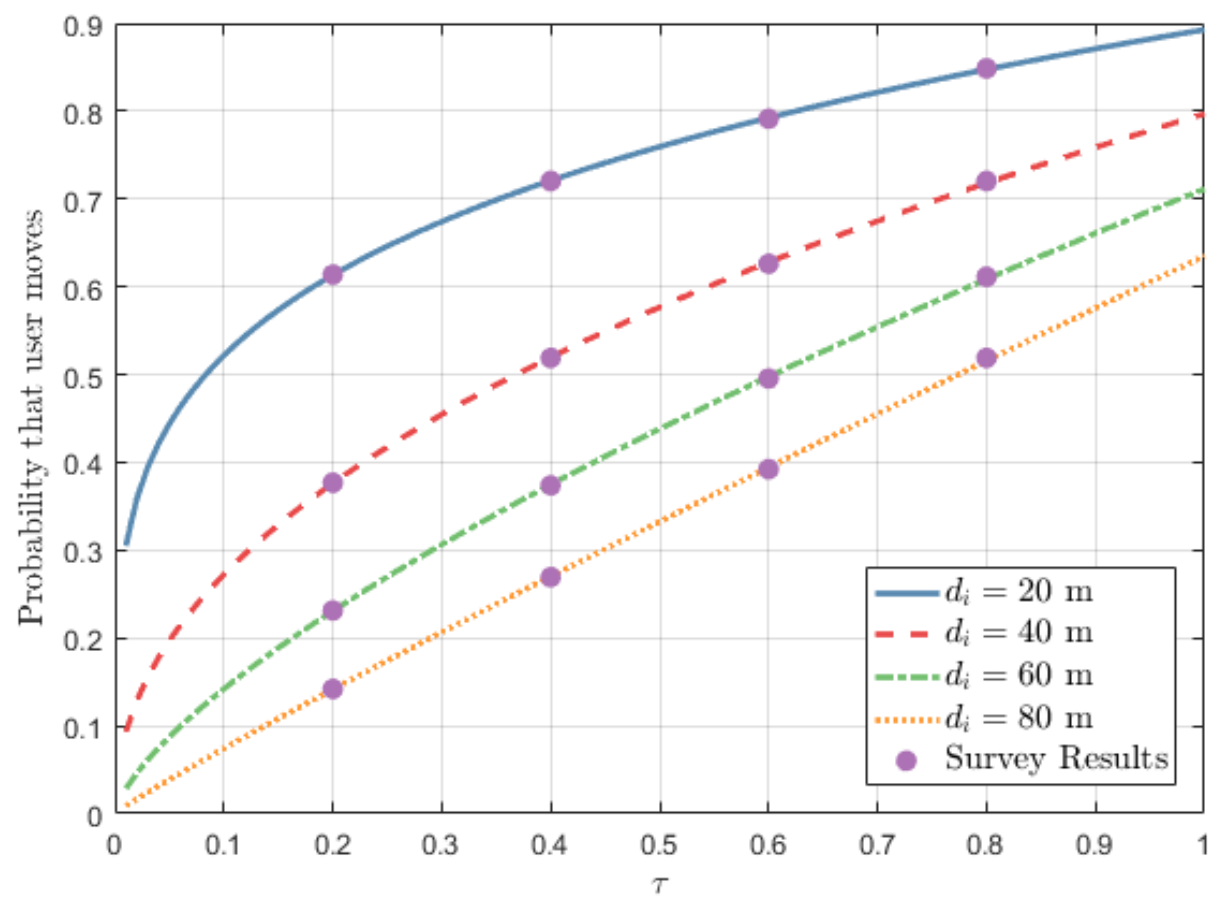

Figure 3.2: Probability of a user accepting to move with respect to incentive (percentage of price discount) and distance: Survey data is fit to $\beta(\tau)$ in (3.2).

highly valuable to the user at that moment, e.g., a video conference call, or the application will be running for a while. By utilizing big data (history) and machine learning technologies, the incentivized move suggestions can be made user/context/situation-aware [176]. However, there is not an existing tractable model to incorporate all these aspects.

As discussed earlier in Section 2.1.1, the SNC parameters not only include UIL, but also involve dynamic placement of a drone-BS. In this case, the model in [192] provides simplicity and tractability. SNC parameters can either be determined separately by considering drone-BS and UIL systems in an uncoordinated fashion, or they can be determined jointly.

\subsection{Uncoordinated Spatial Network Configuration}

A system incorporating drone-BSs and UIL requires handling two processes for spatial network coordination: 
- 3-D placement of a drone-BS, i.e., determining $\left(x_{D}, y_{D}, h_{D}\right)$, and

- Incentive design for user-in-the-loop, i.e., determining $\tau_{i}$ and $d_{i}$ for each user $i \in \mathbb{U}$.

The method of handling these processes in a sequential manner is termed uncoordinated spatial network coordination (USNC). In the following sections, both steps will be discussed in detail.

\subsubsection{3-D Placement of a Drone-BS}

For the sake of completeness and consistency of notation hereafter, Section 2.1.2 is represented here. Recall that, placement of a drone-BS is different from terrestrial BS placement because of the following reasons:

1. In addition to choosing the drone-BS's location in the horizontal space $\left(x_{D}, y_{D}\right)$, we need to determine its altitude, $h_{D}$, as well.

2. The coverage area of a terrestrial BS is known a priori. However, the coverage area of a drone-BS depends on its altitude, and is unknown before solving the placement problem.

3. The mobility of the drone-BS allows it to move wherever the demand is, rather than terrestrial cells waiting for the demand to come towards them. As a result, the coverage region providing the maximum benefit to the network should be found.

The first item indicates that the placement of the drone-BS is a 3-D problem. In addition, the last two items, which are determining the size of the coverage area, and identifying the location of the coverage area must be considered jointly. We assume that a user is in the coverage region of the drone-BS if the air-to-ground link satisfies its QoS requirement. Hence, user $i$ is served by the drone-BS, if $L\left(h_{D}, r_{i}\right) \leq \gamma$. Using (2.3), we can re-write this condition as

$$
h_{D}^{2}+r_{i}^{2} \leq 10^{\frac{\gamma-\left(z_{1} P\left(h_{D}, r_{i}\right)+z_{2}\right)}{10}} .
$$

Let $u_{i} \in\{0,1\}$ denote a binary variable that indicates whether user $i$ is served by the drone$\mathrm{BS}$, or not. Using the variable $u_{i}$, which is equal to 1 , only if the user $i$ is served by the 
drone-BS, and equal to 0 otherwise, the following constraint,

$$
u_{i}\left(h_{D}^{2}+r_{i}^{2}\right) \leq 10^{\frac{\gamma-\left(z_{1} P\left(h_{D}, r_{i}\right)+z_{2}\right)}{10}}
$$

determines whether user $i$ is covered, or not. This constraint can be further manipulated to

$$
\sqrt{h_{D}^{2}+r_{i}^{2}} \leq \sqrt{10^{\frac{\gamma-\left(z_{1} P\left(h_{D}, r_{i}\right)+z_{2}\right)}{10}}}+M_{1}\left(1-u_{i}\right)
$$

where $M_{1}$ is a constant that is sufficiently larger than the maximum possible value of the distance between a user and the drone-BS. Observe that when $u_{i}=1,(3.5)$ is equivalent to (3.3). If $u_{i}=0$, since $M_{1}$ is large enough, this constraint is released. Now, we can continue by determining the objective function.

Assuming a fixed QoS for all users, the best region to be served by the drone-BS is identified with the maximum number of users covered. By using (3.5), the placement problem can be written as

$$
\begin{array}{ll}
\operatorname{maximize}_{x_{D}, y_{D}, h_{D},\left\{u_{i}\right\}} \quad \sum_{i \in \mathbb{U}} u_{i} & \\
\text { s.t. } & \sqrt{h_{D}^{2}+r_{i}^{2}} \leq \sqrt{10^{\frac{\gamma-\left(z_{1} P\left(h_{D}, r_{i}\right)+z_{2}\right)}{10}}}+M_{1}\left(1-u_{i}\right), \\
& \forall i=1, \ldots,|\mathbb{U}|, \\
& x_{l} \leq x_{D} \leq x_{u}, \\
y_{l} \leq y_{D} \leq y_{u}, & \\
h_{l} \leq h_{D} \leq h_{u}, & \\
u_{i} \in\{0,1\}, & \forall i=1, \ldots,|\mathbb{U}|,
\end{array}
$$

where $|\cdot|$ represents the cardinality of a set, subscripts $(\cdot)_{l}$ and $(\cdot)_{u}$ denote respectively the minimum and maximum allowed values for $x_{D}, y_{D}$, and $h_{D}$ of the drone-BS. Note that there are quadratic, exponential and binary terms in this problem, which makes it a mixed integer non-linear problem (MINLP). We will show that this problem can be solved efficiently by using a combination of the interior-point optimizer of MOSEK solver and bisection search.

Let $R$ denote the radius of the area to be covered by the drone-BS. Then, if the user $i$ is covered, $r_{i} \leq R$ must be satisfied, i.e., the served user must be located within the coverage 
region. Let $\alpha$ denote the ratio of the altitude to the coverage region, such that

$$
\alpha=\frac{h_{D}}{R}
$$

Then, (3.5) can be re-organized by using (3.7)

$$
r_{i} \leq \Gamma(\alpha)-M_{2}\left(1-u_{i}\right)
$$

where $M_{2}$ is a constant similar to $M_{1}$, and

$$
\Gamma(\alpha)=\sqrt{\frac{10^{\frac{\gamma-\left(z_{1} P(\alpha)+z_{2}\right)}{10}}}{\left(1+\alpha^{2}\right)}},
$$

where

$$
P(\alpha)=\frac{1}{1+a \exp (-b(\arctan (\alpha)-a))}
$$

by (2.1).

Proposition 2. For any $Q o S$ requirement, $\gamma$, and for any operating frequency, $f_{c}$, if a local maxima, $\Gamma^{*}$, exists in the function $\Gamma(\alpha)$ defined in (3.9), then it is the only local maxima of the function for $\alpha \in(0, \infty)$ for the propagation environments whose parameters are listed in Table 2.5. The $\alpha^{*}$ yielding $\Gamma^{*}$ is the solution of

$$
\alpha \pi\left(a \mathrm{e}^{-\left(\frac{180 \tan ^{-1}(\alpha)}{\pi}-a\right)}+1\right)^{2}-k_{3} \mathrm{e}^{-b\left(\frac{180 \tan ^{-1}(\alpha)}{\pi}-a\right)}=0,
$$

where $k_{3}=-9 \ln (10) a b z_{1}$.

Proof. See Conjecture 1.

The largest feasible set for Problem (2.7) can be obtained by substituting $\Gamma(\alpha)$ with $\Gamma^{*}$, which is the only maxima of $\Gamma(\alpha)$. Since no closed-form expression is available by (3.11), bisection search can be used to obtain a numerical value for $\alpha^{*}$. Then, substituting $\Gamma(\alpha)$ with $\Gamma^{*}$ yields the following mixed integer quadratically constrained problem (MIQCP)

$$
\begin{array}{ll}
\underset{x_{D}, y_{D},\left\{u_{i}\right\}}{\operatorname{maximize}} & \sum_{i \in \mathbb{U}} u_{i} \\
\text { s. t. } r_{i} \leq \Gamma^{*}+\left(1-u_{i}\right) M_{2}, & \forall i=1, \ldots,|\mathbb{U}|,
\end{array}
$$




$$
\begin{aligned}
& x_{l} \leq x_{D} \leq x_{u}, \\
& y_{l} \leq y_{D} \leq y_{u}, \\
& u_{i} \in\{0,1\},
\end{aligned}
$$

While $R$ can be derived from the location of the covered user with maximum $r_{i}, h_{D}$ can be calculated by (3.7). Hence, $h_{D}$ is omitted from (3.12) as a variable. Therefore, both complexity of calculation, and the number of variables are reduced. Then, this problem can be solved efficiently by using interior-point optimizer of MOSEK. The above problem formulation yields the optimal position of the drone-BS. Once the drone-BS is positioned, $d_{i}$ can be calculated for each non-covered user, and incentives can be designed to increase revenue.

\subsection{Incentive Design for User-in-the-loop}

Based on the UIL scheme in Section 3.2.1, the average unit profit obtained from a user at a distance $d_{i}$ meters from the coverage region after receiving incentive $\tau_{i}$ is

$$
\Pi\left(\tau_{i}, d_{i}\right)=\left(1-\tau_{i}\right) \mathrm{e}^{-\beta\left(\tau_{i}\right) d_{i}} .
$$

Proposition 3. For an uncovered user at a ground distance $d_{i}$ from the coverage area of the drone-BS, the optimal incentive that maximizes $\Pi\left(\tau_{i}, d_{i}\right), \tau_{i}^{*}$, is given by

$$
\tau_{i}^{*}=\frac{k_{1} d_{i}}{k_{1} d_{i}-1} .
$$

Proof. For a given $d_{i}$, the stationary point of $\Pi\left(\tau_{i}, d_{i}\right), \tau_{i}^{*}$, can be evaluated by taking the first derivative of (3.13) and equating it to zero, which yields (3.14). The second derivative of (3.13) is given by

$$
\mathrm{e}^{-k_{2} d_{i}} k_{1} d_{i} \tau_{i}^{-\left(k_{1} d_{i}+1\right)}\left(\left(k_{1} d_{i}+1\right) \tau_{i}^{-\left(k_{1} d_{i}+1\right)}-\left(k_{1} d_{i}-1\right)\right) .
$$

It can be shown that (3.15) is negative for all values of $d_{i}$ at $\tau_{i}^{*}$. Hence, $\Pi\left(\tau_{i}, d_{i}\right)$ is nondecreasing for $\tau_{i} \leq \tau_{i}^{*}$, and nonincreasing for $\tau_{i} \geq \tau_{i}^{*}$ for all $\tau_{i} \in(0,1]$, which satisfies the second-order condition for quasiconcavity of $\Pi\left(\tau_{i}, d_{i}\right)$ in $\tau_{i}$ for all values of $d_{i}$ [198]. 


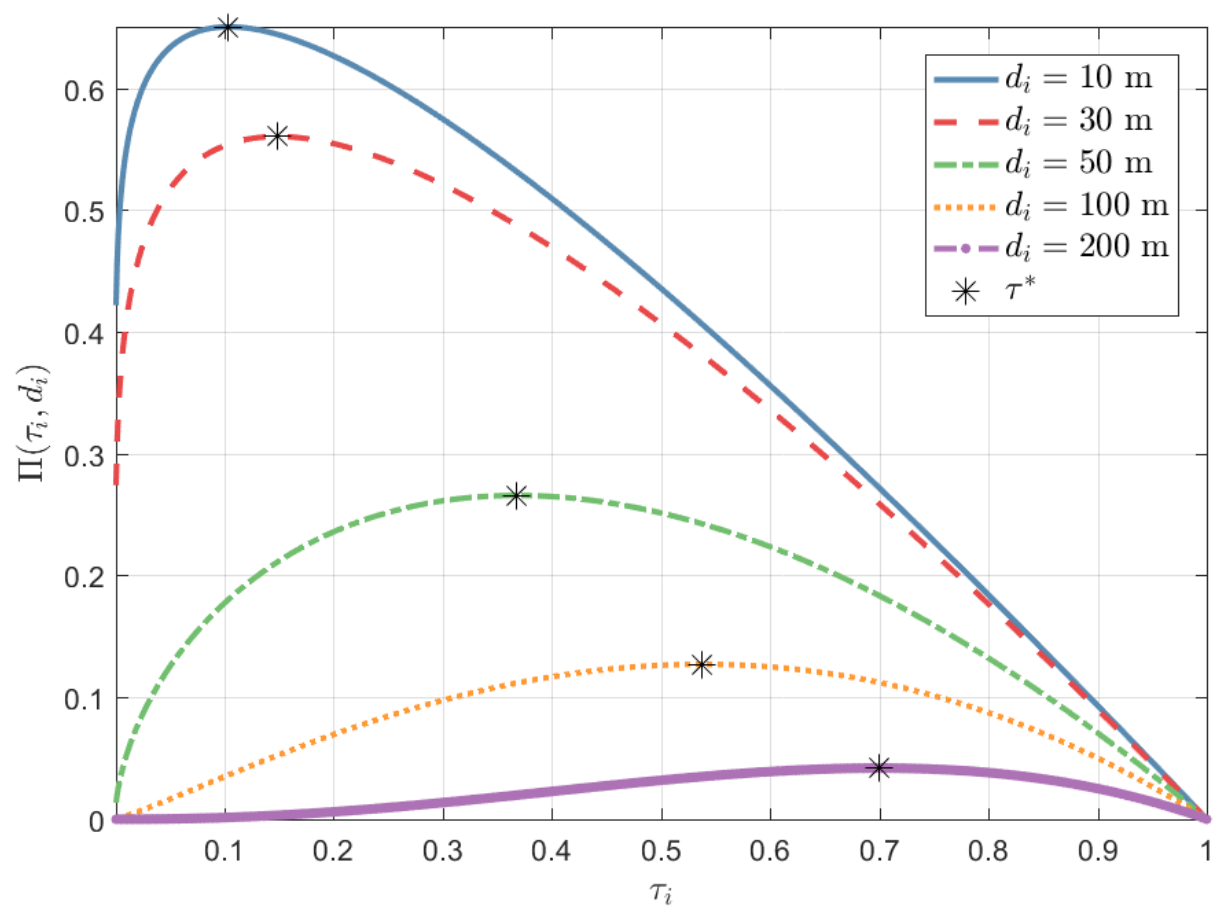

Figure 3.3: Unit profit vs. incentive for several distances.

Therefore, $\tau_{i}^{*}$ is the global maximum point of (3.13).

Once the drone-BS is placed as explained in Section 3.3.1, $d_{i}$ can be calculated, and the above analysis can be used to offer the optimal incentive to each user. In Fig. 3.3, $\Pi\left(\tau_{i}, d_{i}\right)$ is shown for users at several distances. It can be observed that the users in proximity can become profitable with small incentive offers, however, the users that are far from the drone-BS do not provide much gain. For instance, $10.4 \%$ discount offer can provide $65 \%$ average profit for a user that should move $10 \mathrm{~m}$. On the other hand, offering $53.8 \%$ discount can only provide $12.7 \%$ average profit for a user that should move $100 \mathrm{~m}$, as the likelihood of accepting the incentive is very low. Therefore, it can be critical to position the droneBS by considering the persuasion parameter and profitability from UIL, which is discussed next. 


\subsection{Regional Analysis on Coverage and Optimal Incentive}

Coverage of a drone-BS is investigated in several studies, e.g., [116] and [28], however, UIL is not considered in any of the studies so far. As discussed in Section 3.2.1, there is a probability that the user will move from $\mathcal{D}$ to $\mathcal{R}$, which means that the previously uncovered users can be covered under certain circumstances.

Regional analysis can be useful when the location information of users is not available, hard to obtain, or incorrect, when the users are highly mobile such that the coherence time of snapshot analysis is very low, or when the profitability of the region is to be investigated for a known user density.

\subsubsection{Extended Coverage of a BS in a System with UIL}

The study in this section applies to both terrestrial and aerial BSs. Let $\mathrm{P}^{\mathrm{R}}$ denote probability of coverage for a user $i$ in $\mathcal{R}$. Since users are uniformly distributed, and a user is assumed to be covered if it is in $\mathcal{R}$,

$$
\begin{aligned}
\mathrm{P}^{\mathrm{R}} & =\mathrm{P}\left\{u_{i}=1 \cap\left(x_{i}, y_{i}\right) \in \mathcal{R}\right\} \\
& =\frac{R^{2}}{W^{2}},
\end{aligned}
$$

where $u_{i}=1$ indicates that the user $i$ is covered, and $u_{i}=0$ indicates the opposite.

The users within range $D$ can also be covered, if they accept the incentive and move in $\mathcal{R}$. Let $\mathrm{P}^{\mathrm{D}}$ denote the probability of a user $i$ to be covered in $\mathcal{D}$,

$$
\begin{aligned}
\mathrm{P}^{\mathrm{D}} & =\mathrm{P}\left\{u_{i}=1 \cap\left(x_{i}, y_{i}\right) \in \mathcal{D}\right\} \\
& =\int_{R}^{R+d_{u}} \frac{2 \pi r}{\pi W^{2}} \mathrm{e}^{-\beta(\tau)(r-R)} d r \\
& =\frac{2\left(\left(-\beta(\tau)\left(R+d_{u}\right)-1\right) \mathrm{e}^{-\beta(\tau)\left(d_{u}\right)}+\beta(\tau) R+1\right)}{W^{2} \beta^{2}(\tau)}
\end{aligned}
$$

Then, coverage probability of any user in $\mathcal{W}$, i.e., $\left(x_{i}, y_{i}\right) \in \mathcal{W}$, can be obtained from (3.16) 
and (3.17) as

$$
\mathrm{P}^{\mathrm{W}}=\mathrm{P}^{\mathrm{R}}+\mathrm{P}^{\mathrm{D}}
$$

The coverage probabilities calculated above, help analysing profitability of a region, as discussed in the following section.

\subsubsection{Optimal Regional Incentive}

The profit of the network operator is the difference between the income from all the covered users, and the cost of the incentives. Assuming all users in $\mathcal{D}$ receive the same incentive, which is the regional incentive, $\tau$, the average normalized revenue per user is

$$
\Pi_{\circ}(\tau)=\mathrm{P}^{\mathrm{R}}+\mathrm{P}^{\mathrm{D}}(1-\tau)
$$

The percentage of the gain from UIL, which is $100 \times \frac{\mathrm{P}^{\mathrm{D}}(1-\tau)}{\mathrm{P}^{\mathrm{R}}}$, is plotted in Fig. 3.4. As can be observed from Fig. 3.4, an optimal regional incentive maximizing the profit, $\tau^{*}$, can be obtained. The main reason for this behaviour is that the probability of moving for a user (given in (3.1)) does not increase significantly even if more incentive is offered. The optimal regional incentive $\tau^{*}$ can be obtained by solving $\frac{d \Pi_{\mathrm{o}}(\tau)}{d \tau}=0$, which is the solution of

$$
\begin{array}{r}
2 \beta(\tau) k_{1}\left(-d_{u} \mathrm{e}^{-\beta(\tau) d_{u}}\left(\left(R+d_{u}\right)(-\beta(\tau)-1)\right)-\left(R+d_{u}\right) \mathrm{e}^{-\beta(\tau) d_{u}}+R\right)- \\
4 k_{1}\left(\mathrm{e}^{-\beta(\tau) d_{u}}\left(\left(R+d_{u}\right)(-\beta(\tau)-1)\right)+R \beta(\tau)+1\right)=0
\end{array}
$$

Again from Fig. 3.4, we observe that both the gain from the UIL and $\tau^{*}$ increases with increasing $d_{u}$ for a given coverage radius, $R$. Therefore, in Fig. 3.5, we consider $d_{u} \rightarrow W-R$ for a given $R$. It means that $d_{u}$, and, hence, $\mathcal{D}$ is as large as possible, which should lead to maximum gain from UIL. We observe that $\tau^{*}$ saturates to a certain value, $\tau^{\infty}$, which will be obtained by analysing limiting conditions of (3.19) next.

First, note that the average normalized revenue in a region with $N$ users is

$$
\Pi_{N}(\tau)=N \times \Pi_{\circ}(\tau)
$$

In order to obtain an infinite region, consider $W \rightarrow \infty$ and $d_{u} \rightarrow W-R$. Then, the limit of 


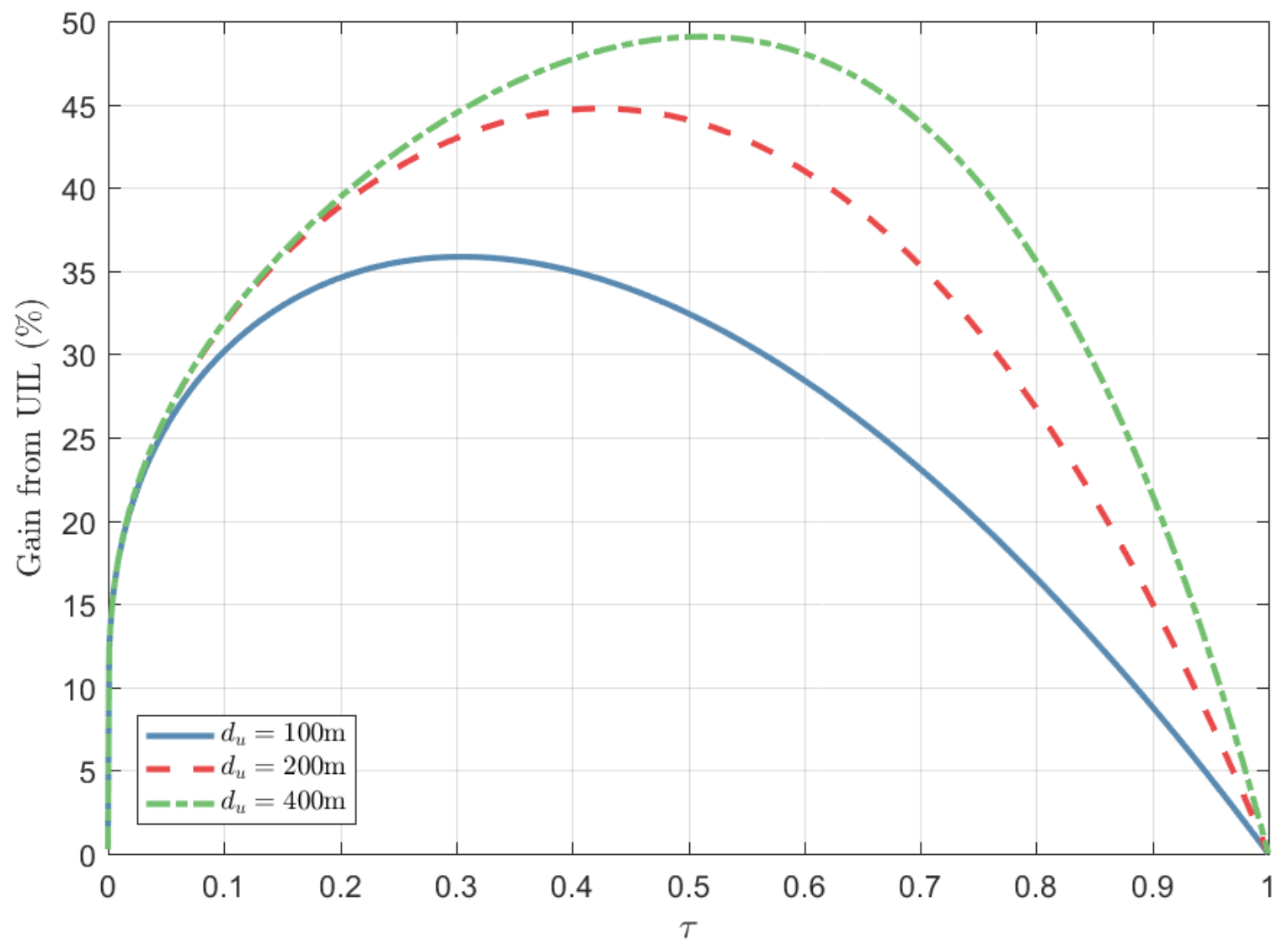

Figure 3.4: Gain from the UIL with respect to regional incentive, $\tau$ is calculated for $R=$ $200, W=2000$.

$\mathrm{P}^{\mathrm{R}} \rightarrow 0$, as can be observed from (3.16). Let $\Pi_{N}^{\infty}(\tau)$ denote the limit of $\Pi_{N}(\tau)$ such that

$$
\lim _{\substack{W \rightarrow \infty, d_{u} \rightarrow W-R}} \Pi_{N}(\tau)=\Pi_{N}^{\infty}(\tau)
$$

For a fixed user density, $\lambda$, substituting $N=\lambda \pi W^{2}$, results

$$
\Pi_{N}^{\infty}(\tau)=\lambda \pi\left(R^{2}+\frac{2(1-\tau)}{\beta^{2}(\tau)}(-\beta(\tau) R+1)\right)
$$

Then, the optimal regional incentive maximizing $\Pi_{N}^{\infty}(\tau), \tau^{\infty}$, is simply the solution of $\frac{d \Pi^{\infty}(\tau)}{d \tau}=0$, which is

$$
\frac{k_{1} R(1-\tau)}{\tau \beta^{2}(\tau)}-\frac{R \beta(\tau)+1}{\beta^{2}(\tau)}+\frac{2 k_{1}(1-\tau)(-R \beta(\tau)+1)}{\tau \beta^{3}(\tau)}=0
$$




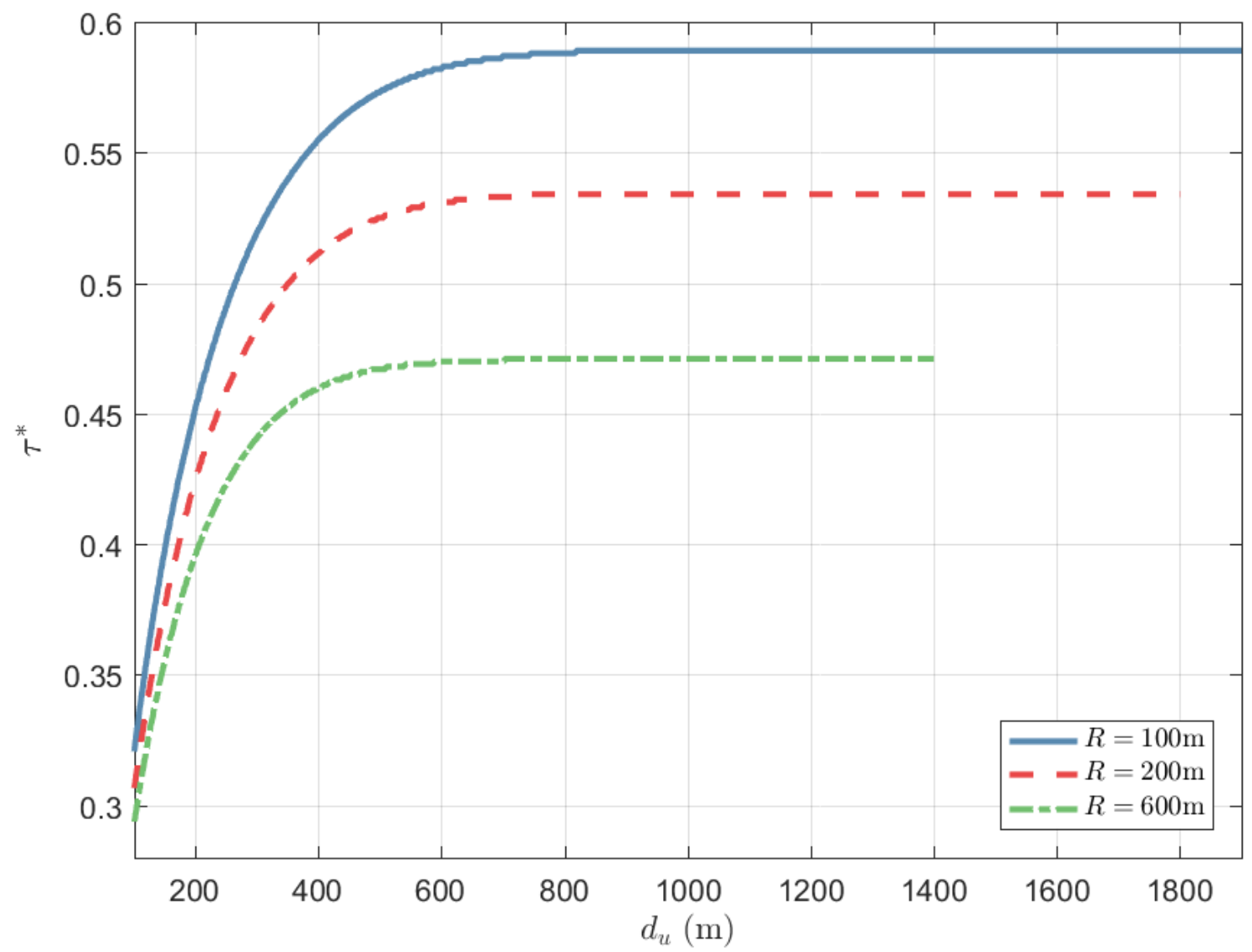

Figure 3.5: Optimal regional incentive is calculated with respect to $d_{u}$ for $R=$ $\{100,200,600\}, W=2000$.

Note that, $\Pi_{N}^{\infty}\left(\tau^{\infty}\right)$ represents the maximum gain from UIL for any given coverage radius, $R$, and user density, $\lambda$. Moreover, $\Pi_{N}^{\infty}\left(\tau^{\infty}\right)$ is valid for any base station, either terrestrial or aerial. In case of drone-BSs, $\Pi_{N}^{\infty}\left(\tau^{\infty}\right)$ can be used to analyse the cost of expanding the coverage region, which can either be performed by increasing the transmit power, which may lead to changing the type of the utilized drone, required legal permissions and so on [2], or simply offering more incentives to the users.

\subsection{Joint Spatial Network Configuration}

Differently from the previous section, a joint spatial network configuration (JSNC) problem is proposed to simultaneously position the drone-BS in 3-D and determine the incentive to be offered to each user. Hence, mobility of both the drone-BS and users can be jointly 
considered as degrees of freedom of the spatial configuration of mobile networks.

In order to make UIL an inherent part of the placement problem, the coverage condition in the first constraint of (3.12) can be modified as

$$
r_{i}-d_{i} \leq \Gamma^{*}+u_{i}\left(1-M_{3}\right)
$$

where $M_{3}$ is a sufficiently larger value than the maximum possible value of the right-handside (RHS) of (3.25). In contrast to the approach in Section 3.3.1, maximizing the number of covered users does not necessarily mean maximizing profit this time, because additional users come at a cost with UIL. In fact, the coverage condition in (3.25) yields three regions in a circular area, $\mathcal{W}$, as shown in Fig. 3.6:

1. The circular coverage area of the drone-BS at $\left(x_{D}, y_{D}\right)$ with radius $R$ is defined as the region $\mathcal{R}$.

2. The shaded area, $\mathcal{D}$, is where the UIL model is utilized. It is obtained by excluding $\mathcal{R}$ from a concentric circular area with the radius of $R+d_{u}$, where $d_{u}$ is an upper bound on the distance of a user from the coverage area of the drone-BS such that the users further than $d_{u}$ meters do not receive incentive offers.

3. $\mathcal{W}-(\mathcal{R} \cup \mathcal{D})$ is the region that cannot be served by the drone-BS even with UIL.

Note that, (2.3) yields a circular coverage area for a drone-BS, as well as, designing incentives based on $d_{i}$ yields a circular expansion area by UIL (in Section 3.2.1). Hence, a homogeneous environment is maintained. Further analysis on coverage of a drone-BS in a system with UIL and regional incentive design for uniformly distributed users can be found in Section 3.5.

The profit obtained from a user $i$ based on the coverage region can be written as

$$
\Upsilon\left(\tau_{i}, d_{i}\right):=\left\{\begin{array}{lc}
1, & \text { if }\left(x_{i}, y_{i}\right) \in \mathcal{R}, \\
\Pi\left(\tau_{i}, d_{i}\right), & \text { if }\left(x_{i}, y_{i}\right) \in \mathcal{D}, \\
0, & \text { o.w. }
\end{array}\right.
$$

where (3.26), (3.27) and (3.28) indicate the users covered in $\mathcal{R}$, coverage in $\mathcal{D}$, and outage, 


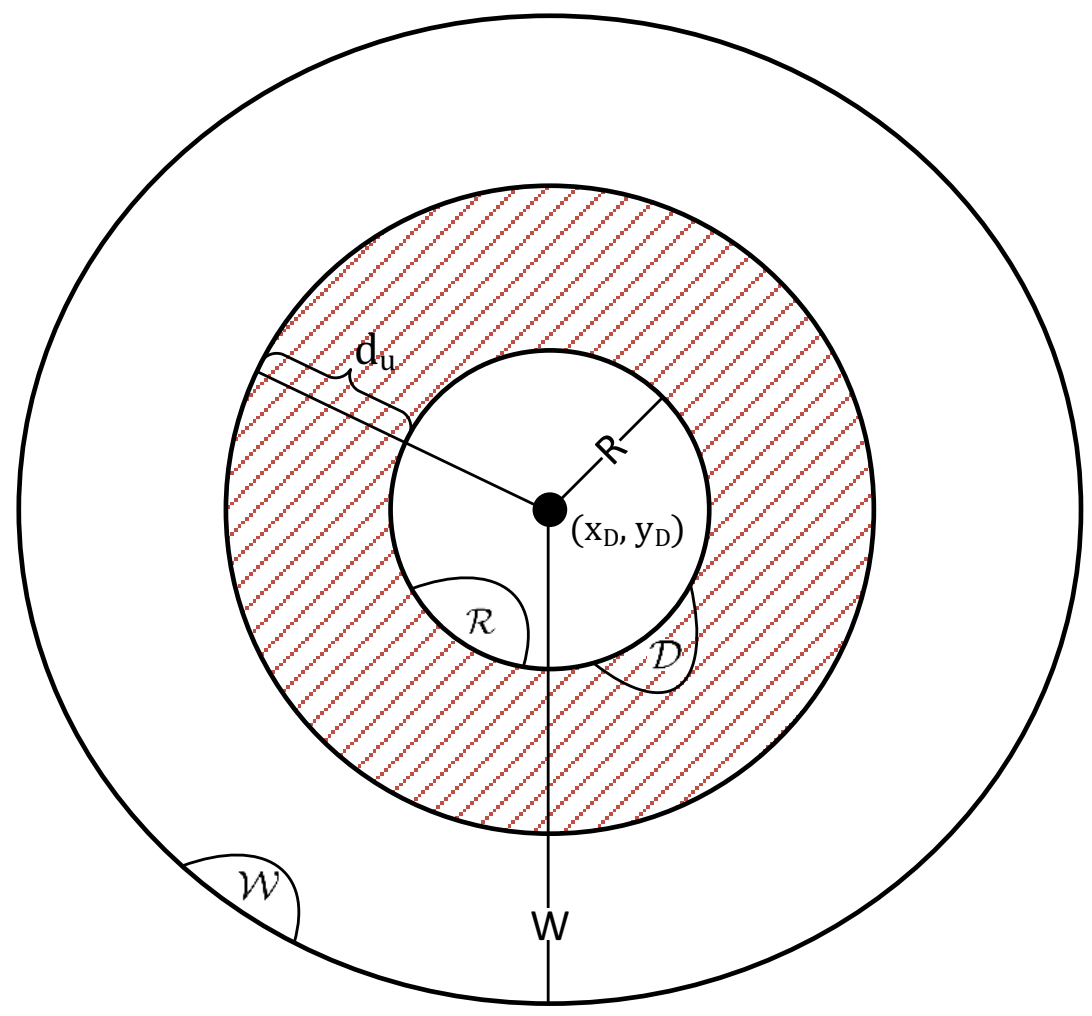

Figure 3.6: Coverage area of a drone-BS $(\mathcal{R})$, area where the UIL model is utilized $(\mathcal{D})$, and a finite space where users are distributed $(\mathcal{W})$.

respectively. Hence, a JSNC problem for maximizing profit can be formulated as

$$
\begin{aligned}
& \underset{x_{D}, y_{D},\left\{u_{i}, d_{i}, \tau_{i}\right\}}{\operatorname{maximize}} \sum_{i \in \mathbb{U}} \Upsilon\left(\tau_{i}, d_{i}\right) \\
& \text { s. t. } r_{i}-d_{i} \leq \Gamma^{*}+M_{3}\left(1-u_{i}\right), \quad \forall i=1, \ldots,|\mathbb{U}|, \\
& u_{i} d_{i} \leq d_{u}, \quad \forall i=1, \ldots,|\mathbb{U}|, \\
& x_{l} \leq x_{D} \leq x_{u} \text {, } \\
& y_{l} \leq y_{D} \leq y_{u}, \\
& u_{i} \in\{0,1\}, \quad \forall i=1, \ldots,|\mathbb{U}|,
\end{aligned}
$$

where $d_{u}$ represents the upper bound for the amount of displacement. Similarly to (3.6), $M_{3}$ in (3.29a) relaxes the condition of coverage, if the user is too far to be covered. If a user is persuaded to move towards the coverage region of drone-BS, (3.29a) ensures that 
the user satisfies the condition of coverage in (3.25). Note that the actual displacement of the user is $\sqrt{\left(x_{i}-\Delta x_{i}\right)^{2}+\left(y_{i}-\Delta y_{i}\right)^{2}}$. However, instead of $\Delta x_{i}$ and $\Delta y_{i}, d_{i}$ indicating the amount of movement is used to decrease number of variables in the problem. Once the problem is solved, $\Delta x_{i}$ and $\Delta y_{i}$ can obtained from $\left(x_{i}, y_{i}\right)$ and $\left(x_{D}, y_{D}\right)$. In order to exploit the benefits from UIL as much as possible, there is no constraint on normalized incentives, $\tau_{i}$. However, scarcity of available incentives can be considered with a constraint as follows

$$
\sum_{i \in \mathbb{U}} \tau_{i} \leq v
$$

Although the constraint in (3.30) is not analytically complex, it results in an interesting problem by adding another dimension to the problem: The incentive design not only depends on the spatial locations of the users, but also the scarcity of the financial resources is considered. For instance, users at the same distance to the drone-BS are offered the same incentives with the current formulation. If the constraint in (3.30) is added to the problem formulation, and there is scarcity of incentives, one of those users at the same distance may not even receive incentives, or, both of them may receive a lower amount than what they would get with the former formulation. The selection between these two option depends on the trade-off of the persuasion parameter and maximizing profit. In order to keep the problem focused on exploiting the SNC framework as much as possible, we do not consider (3.30) for now. Unfortunately, the problem in (3.29) is not efficiently solvable, due to (3.27), which is a non-linear multivariate function, and requires further manipulations.

The triangle method is one of the most popular methods for piecewise linear approximations of multivariate functions [199]. For $\Pi\left(\tau_{i}, d_{i}\right)$, the triangle method partitions the $\tau$ and $d$ axes into $m$ and $n$ sampling intervals, respectively. Let $\left\{\tilde{\tau}_{k}\right\}_{k=1}^{m}$ and $\left\{\tilde{d}_{j}\right\}_{j=1}^{n}$ represent incentive and displacement vertices, respectively. In order to approximate a given $\Pi\left(\tau_{i}, d_{i}\right)$, the rectangular area with vertices satisfying $\tilde{\tau}_{k} \leq \tau_{i} \leq \tilde{\tau}_{k+1}$, and $\tilde{d}_{j} \leq d_{i} \leq \tilde{d}_{j+1}$ is found for $k=1, \ldots, m$ and $j=1, \ldots, n$. As shown in Fig. 3.7, the rectangular area can be divided into two triangles, $T_{1}$ and $T_{2}$, with the vertices given by $\left\{\left(\tilde{\tau}_{k}, \tilde{d}_{j}\right),\left(\tilde{\tau}_{k}, \tilde{d}_{j+1}\right),\left(\tilde{\tau}_{k+1}, \tilde{d}_{j+1}\right)\right\}$ and $\left\{\left(\tilde{\tau}_{k}, \tilde{d}_{j}\right),\left(\tilde{\tau}_{k+1}, \tilde{d}_{j}\right),\left(\tilde{\tau}_{k+1}, \tilde{d}_{j+1}\right)\right\}$. Only one of the triangles contain $\left(\tau_{i}, d_{i}\right)$. Therefore, the $T_{1}$ in Fig. 3.7 is chosen if the following is true,

$$
d_{i}>\tilde{d}_{j}+\left(\tau_{i}-\tilde{\tau}_{k}\right) \frac{\tilde{d}_{j+1}-\tilde{d}_{j}}{\tilde{\tau}_{k+1}-\tilde{\tau}_{k}},
$$

and $T_{2}$ is chosen otherwise. 


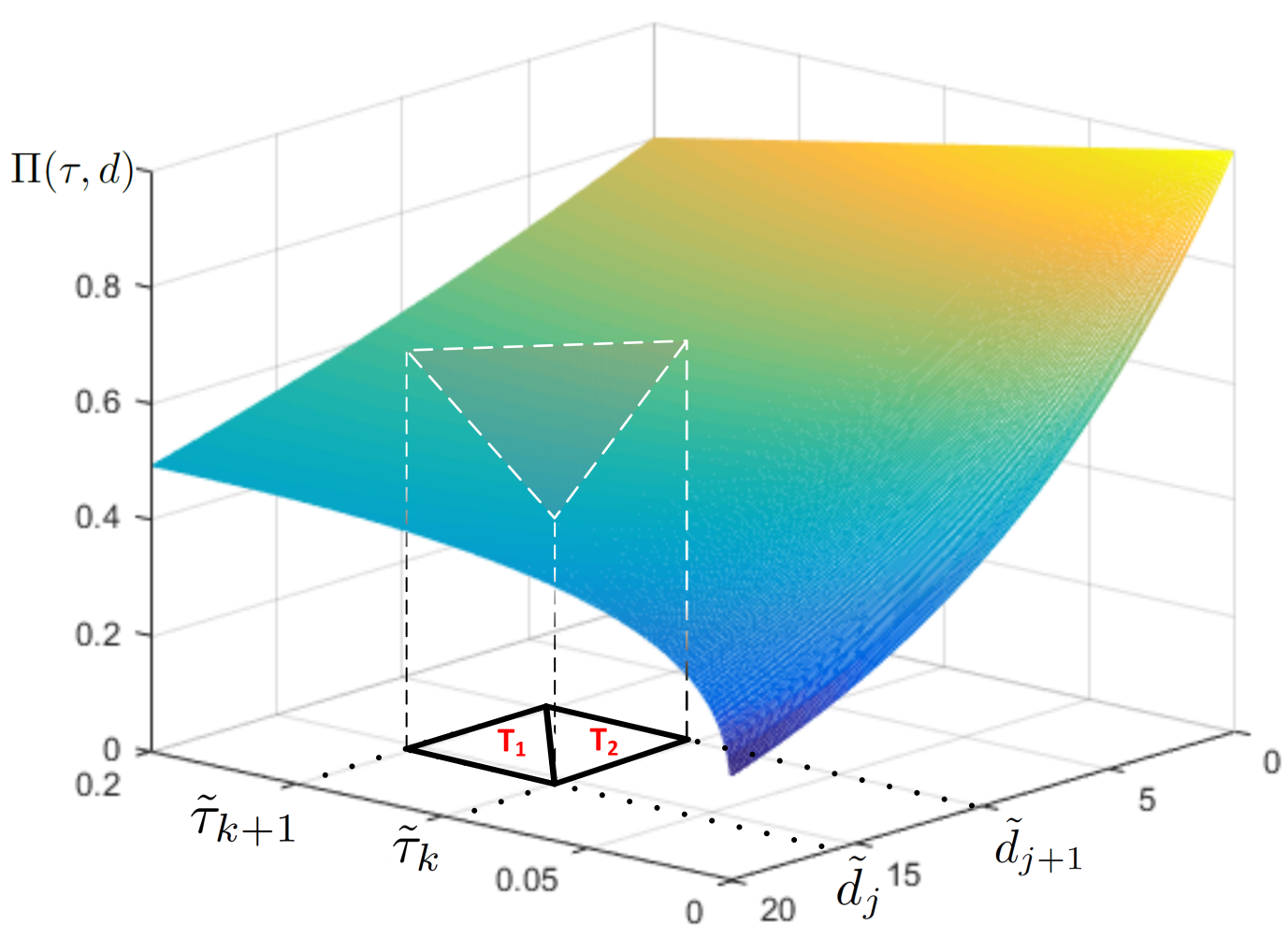

Figure 3.7: Illustration of the triangle method on a portion of $\Pi(\tau, d)$.

In general, the point to be approximated for each user $i$ can be anywhere in the search space, and the approximation points are unknown. Therefore, all the points on the grid must be considered. A mixed integer linear program (MILP) can be formulated to approximate $\Pi\left(\tau_{i}, d_{i}\right)$ :

$$
\begin{array}{ll}
\sum_{k=1}^{n} \sum_{j=1}^{m} \rho_{i, k, j} \tilde{\tau}_{k}=\tau_{i}, & \forall i=1, \ldots,|\mathbb{U}|, \\
\sum_{k=1}^{n} \sum_{j=1}^{m} \rho_{i, k, j} \tilde{d}_{j}=d_{i}, & \forall i=1, \ldots,|\mathbb{U}|, \\
\sum_{k=1}^{n} \sum_{j=1}^{m} \rho_{i, k, j} \Pi\left(\tilde{\tau}_{k}, \tilde{d}_{j}\right)=\Pi\left(\tau_{i}, d_{i}\right), & \forall i=1, \ldots,|\mathbb{U}|,
\end{array}
$$

where $\rho_{i, k, j}$ indicate continuous variables that are introduced for each sampling point. In order to choose three indices corresponding to a triangle, $\rho_{i, k, j}$ must be a special ordered 
set of type 3 (SOS3), which means that only 3 consecutive elements of the set can be non-zero [200]. Also for a user $i$,

$$
\sum_{k=1}^{n} \sum_{j=1}^{m} \rho_{i, k, j}=1
$$

and each $\rho_{i, k, j} \in[0,1]$.

For instance, considering Fig. 3.7, $\Pi\left(\tau_{i}, d_{i}\right)$ can be approximated by the following convex combination of the values of $\Pi(\tau, d)$ at the vertices of the chosen triangle,

$$
\rho_{i, k, j} \Pi\left(\tilde{\tau}_{k}, \tilde{d}_{j}\right)+\rho_{i, k+1, j+1} \Pi\left(\tilde{\tau}_{k+1}, \tilde{d}_{j+1}\right)+\rho_{i, \hat{k}, \hat{j}} \Pi(\hat{\tau}, \hat{d})
$$

where the vertices of the diagonal, $\left(\tilde{\tau}_{k}, \tilde{d}_{j}\right)$ and $\left(\tilde{\tau}_{k+1}, \tilde{d}_{j+1}\right)$ are common in both triangles, $(\hat{\tau}, \hat{d})$ indicate the third set of vertices differentiating the $T_{1}$ and $T_{2}$ based on (3.31), and $\rho_{i, \hat{k}, \hat{j}}$ indicate the weights corresponding to these vertices. Formulating the optimization problem for the triangle method does not suffice to approximate the objective function of (3.29) because it only represents (3.27). However (3.26) and (3.28) too must be represented in the problem formulation. For that reason, in addition to $u_{i}$ in (3.29), consider another binary variable, $w_{i}$, indicating whether the user is in $\mathcal{D}$ or not. These two variables, $u_{i}$ and $w_{i}$ can be used to represent all regions in Fig.3.6, such that

- $u_{i}=1$ indicates that the user is in $\mathcal{R}$,

- $w_{i}=1$ indicates that the user is in $\mathcal{D}$,

- $u_{i}+w_{i}=0$ indicates that the user is not covered,

- $u_{i}+w_{i} \leq 1$ ensures that the user is either in $\mathcal{R}$ or in $\mathcal{D}$.

Finally, using triangle method, we can formulate (3.29) as the following optimization problem:

$$
\begin{aligned}
& \underset{\substack{x_{D}, y_{D},\left\{u_{i}, w_{i}, d_{i}, \tau_{i}, \Pi_{i}^{a}\right\},}}{\operatorname{maximize}} \quad \sum_{i \in \mathbb{U}}\left(u_{i}+\Pi_{i}^{a}\right) \\
& \left\{\rho_{i, k, j}, \kappa_{i, k, j}^{1}, \kappa_{i, k, j}^{2}\right\} \\
& \text { s. t. } r_{i} \leq \Gamma^{*}+M\left(1-u_{i}\right), \quad \forall i=1, \ldots,|\mathbb{U}| \text {, } \\
& r_{i}-d_{i} \leq \Gamma^{*}+M\left(1-w_{i}\right), \quad \forall i=1, \ldots,|\mathbb{U}|,
\end{aligned}
$$




$$
\begin{array}{lr}
\sum_{k=1}^{n} \sum_{j=1}^{m} \rho_{i, k, j}=w_{i}, & \forall i=1, \ldots,|\mathbb{U}|, \\
\sum_{k=1}^{n} \sum_{j=1}^{m} \rho_{i, k, j} \tilde{\tau}_{k}=\tau_{i}, & \forall i=1, \ldots,|\mathbb{U}|, \\
\sum_{k=1}^{n} \sum_{j=1}^{m} \rho_{i, k, j} \tilde{d}_{j}=d_{i}, & \forall i=1, \ldots,|\mathbb{U}|, \\
\sum_{k=1}^{n} \sum_{j=1}^{m} \rho_{i, k, j} \Pi\left(\tilde{\tau}_{k}, \tilde{d}_{j}\right)=\Pi_{i}^{a}, & \forall i=1, \ldots,|\mathbb{U}|, \\
\sum_{k=1}^{n-1} \sum_{j=1}^{m-1} \kappa_{i, k, j}^{1}+\kappa_{i, k, j}^{2}=1, & \forall i=1, \ldots,|\mathbb{U}|, \\
\rho_{i, k, j} \leq \kappa_{i, k, j}^{1}+\kappa_{i, k, j}^{2}+\kappa_{i, k, j-1}^{1}+\kappa_{i, k-1, j}^{2} & \forall i=1, \ldots,|\mathbb{U}|, \\
+\kappa_{i, k-1, j-1}^{1}+\kappa_{i, k-1, j-1}^{2}, & (k=1, \ldots, n), \quad(j=1, \ldots, m), \\
u_{i}+w_{i} \leq 1, \quad & \forall i=1, \ldots,|\mathbb{U}|, \\
u_{i}, w_{i} \in\{0,1\}, & \forall i=1, \ldots,|\mathbb{U}|, \\
\rho_{i, k, j} \in[0,1], \quad & \forall i=1, \ldots,|\mathbb{U}|, \\
\kappa_{i, k, j}^{1}, \kappa_{i, k, j}^{2} \in\{0,1\}, & (j=1, \ldots, m), \\
x_{l} \leq x_{D} \leq x_{u}, & \forall i=1, \ldots,|\mathbb{U}|, \\
y_{l} \leq y_{D} \leq y_{u}, & (j=1, \ldots, m),
\end{array}
$$

where $\rho_{i, k, j}$ corresponds to the weights sampling points (3.34), $\kappa_{i, k, j}^{1}$ and $\kappa_{i, k, j}^{2}$, which are 0 at the extremes, allow choosing either $T_{1}$ or $T_{2}$, respectively. Utilizing $\kappa_{i, k, j}^{1}$ and $\kappa_{i, k, j}^{2}$ in constraints $(3.35 \mathrm{~g})$ and $(3.35 \mathrm{~h})$ provides $\rho_{i, k, j}$ variables to form a SOS3 type set. Hence, three vertices forming a triangle can be chosen. Although full formulation is provided here for completeness, $\kappa_{i, k, j}^{1}$ and $\kappa_{i, k, j}^{2}$ are not needed, if a solver allowing special ordered sets is used. Note that, a constraint similar to (3.29b) is not required, because the vertices already limit possible values of $d_{i}$.

Constraints (3.35c) through (3.35f) are used to approximate $\Pi_{i}^{a}$ using the triangle method. Note that, (3.35c) corresponds to (3.33), where the RHS of (3.33) was replaced 
by $w_{i}$. This substitution ensures that the users outside of $\mathcal{D}$, i.e, $w_{i}=0$, neither receive incentives, nor moves, which yields $\Pi_{i}^{a}=0$. In other words, it turns on and off the triangle method, depending on the location of the user. Similarly, $u_{i}$ and $w_{i}$ are used to switch between (3.35a) and (3.35b), based on the location of the user.

The triangular method increases the number of variables by adding $3 \mathrm{~nm}+1$ variables for each user, which makes it computationally costly for a large number of users. On the other hand, approximation quality of the triangle method increases with increasing number of breakpoints [199]. Therefore, an intervening SNC method, which maintains accuracy of JSNC and computational efficiency of USNC, is proposed next.

\subsection{Semi-Joint Spatial Network Configuration}

In Section 3.3, USNC is described, where the 3-D placement of a drone-BS is performed without considering the effect of the users in $\mathcal{D}$ (Fig. 3.6). A brief analysis in Section 3.4 showed that the maximum profit from a user decreases significantly as the distance between the user and drone-BS increases. Therefore, a JSNC method is developed in the preceding section, where the 3-D placement is performed by taking the users outside the potential coverage region of drone-BS into account. However, JSNC yields a complex MINLP, which is computationally costly. Therefore in this section, we discuss a semi-JSNC method, which locates the drone-BS in 3-D by considering the approximate effect of the users in $\mathcal{D}$. Then, optimal incentive is offered to users based on (3.14). In other words, incentive design is not a completely inherent part of semi-JSNC, yet, it is straightforward to design incentives after carefully placing the drone-BSs.

Semi-JSNC aims at maximizing (3.26) described in the preceding section with a slightly different approach on (3.27). The analysis in Section 3.4 reveal that the maximum value of $\Pi\left(\tau_{i}, d_{i}\right)$ for a certain $d_{i}$ can be obtained by substituting $\tau_{i}^{*}$ corresponding to $d_{i}$. Taking (3.14) into account, $\Pi\left(\tau_{i}^{*}, d_{i}\right)$ becomes a uni-variate function of $d_{i}$. Then, a piece-wise 
linear relationship can be obtained

$$
\check{\Pi}\left(d_{i}\right)=\left\{\begin{array}{cc}
p_{1} d_{i}+s_{1}, & 0<d_{i} \leq t_{2}, \\
p_{2} d_{i}+s_{2}, & t_{2}<d_{i} \leq t_{3}, \\
\vdots & \vdots \\
p_{N} d_{i}+s_{N}, & t_{N-1}<d_{i} \leq d_{u}, \\
0, & \text { o.w. }
\end{array},\right.
$$

where $t_{j}$ for $j=1, \ldots, N$ denotes $N$ breakpoints on the $d$-axis, where $t_{1}=0$, and $t_{N}=d_{u}$. The coefficients of the linear equation at each interval are denoted by $p_{j}$ and $s_{j}$. The selection and number of breakpoints determines the accuracy of (3.36). One of the common practices is choosing equally spaced breakpoints. However, adapting the length of the intervals to the characteristics of the function, e.g., taking breakpoints more frequently when the function changes rapidly, can provide better accuracy compared to the same number of equally-spaced breakpoints. In order to measure accuracy of approximations, RMSE is considered widely. An optimization problem to determine breakpoints with the objective of minimizing RMSE can be formulated by using (3.36),

$$
\begin{array}{ll}
\underset{\left\{p_{j}, s_{j}, t_{j}\right\}}{\operatorname{minimize}} & \int\left(\Pi\left(d_{i}\right)-\check{\Pi}\left(d_{i} ; p_{j}, s_{j}, t_{j}\right)\right)^{2} d t \\
\text { s.t. } & 0 \leq t_{j} \leq d_{u}, \quad \forall j=2, \ldots, N-1,
\end{array}
$$

where the first breakpoint, $t_{1}$, and the last breakpoint, $t_{N}$, are given ( 0 and $d_{u}$, respectively, in this case), and (3.40a) ensures that the other breakpoints are within the first and last ones. In Fig. 3.8, piece-wise linear approximations with $N=3$ and $N=4$ breakpoints are shown with the corresponding RMSE values ${ }^{2}$.

A linear optimization problem can be designed for separable functions like (3.36) by introducing continuous variables $\lambda_{1}, \lambda_{2}, \ldots, \lambda_{N}$ for each breakpoint, such that

$$
\sum_{j=1}^{N} \lambda_{j} \Pi\left(t_{j}\right)=\check{\Pi}\left(d_{i}\right)
$$

\footnotetext{
${ }^{2}$ Shape Language Modelling Toolbox for MATLAB is used to obtain the breakpoints. Available online: https://www.mathworks.com/matlabcentral/fileexchange/24443-slm-shape-language-modeling, Accessed: 05 Sept., 2017.
} 


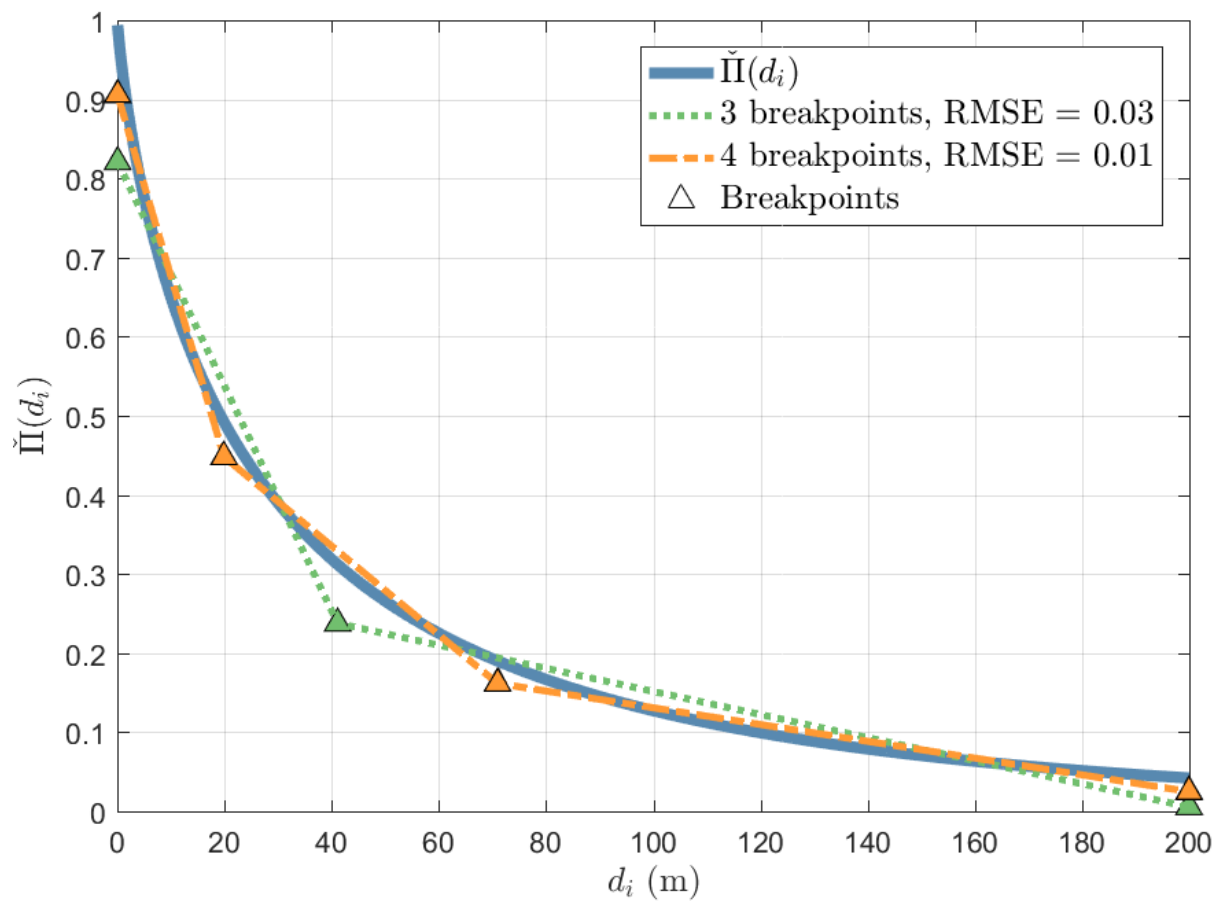

Figure 3.8: Piece-wise approximation with 3 and 4 breakpoints, and the resulting RMSE values, where $d_{u}=200 \mathrm{~m}$.

$$
\begin{aligned}
& \sum_{j=1}^{N} \lambda_{j} t_{j}=d_{i} \\
& \sum_{j=1}^{N} \lambda_{j}=1
\end{aligned}
$$

with the additional requirement that $\left\{\lambda_{i}\right\}_{i=1}^{N}$ are SOS2 type variables. It is required to guarantee that approximated values, $d_{i}$ and $\check{\Pi}\left(d_{i}\right)$ lie on the approximating line segments. Then, making use of the variables $u_{i}$ and $w_{i}$ indicating regions in Fig. 3.6 (in Section 3.6), an efficient optimization problem can be formulated

$$
\begin{aligned}
& \underset{\substack{x_{D}, y_{D},\left\{d_{i}, \tilde{\Pi}\left(d_{i}\right), u_{i}, w_{i}\right\}}}{\operatorname{maximize}} \quad \sum_{i \in \mathbb{U}} u_{i}+\check{\Pi}\left(d_{i}\right) \\
& \left\{\lambda_{i, j}, z_{i, j}\right\} \\
& \text { s.t. } \quad r_{i} \leq \Gamma^{*}+M\left(1-u_{i}\right), \quad \forall i=1, \ldots,|\mathbb{U}| \text {, } \\
& r_{i}-d_{i} \leq \Gamma^{*}+M\left(1-w_{i}\right), \quad \forall i=1, \ldots,|\mathbb{U}|, \\
& \sum_{j=1}^{N} \lambda_{i, j} \Pi\left(t_{j}\right)=\check{\Pi}\left(d_{i}\right), \quad \forall i=1, \ldots,|\mathbb{U}|,
\end{aligned}
$$




$$
\begin{array}{cc}
\sum_{j=1}^{N} \lambda_{i, j} t_{j}=d_{i}, & \forall i=1, \ldots,|\mathbb{U}|, \\
\sum_{j=1}^{N} \lambda_{i, j}=w_{i}, & \forall i=1, \ldots,|\mathbb{U}|, \\
\sum_{j=1}^{N} z_{i, j} \leq 2, & \forall i=1, \ldots,|\mathbb{U}|, \\
\sum_{k=j+2}^{N} z_{i, j}+z_{i, k} \leq 1, & \forall j=1, \ldots, N, \quad \forall i=1, \ldots,|\mathbb{U}|, \\
\lambda_{i, j} \leq z_{i, j}, & \forall j=1, \ldots, N, \quad \forall i=1, \ldots,|\mathbb{U}|, \\
\lambda_{i, j} \in[0,1], & \forall j=1, \ldots, N, \forall i=1, \ldots,|\mathbb{U}|, \\
z_{i, j}, u_{i}, w_{i} \in\{0,1\}, & \forall j=1, \ldots, N, \forall i=1, \ldots,|\mathbb{U}|, \\
x_{l} \leq x_{D} \leq x_{u}, & \\
y_{l} \leq y_{D} \leq y_{u}, &
\end{array}
$$

where $z_{i, j}$ are binary variables used to ensure that $\lambda$ 's are SOS2 variables via constraints (3.44f), (3.44g), and (3.44h). The first two constraints are the same as in (3.35), where $u_{i}$ and $w_{i}$ yield an objective function similar to (3.26). The piece-wise approximation constraints (3.44c) and (3.44d) correspond to (3.41) and (3.42), however, (3.43) is modified to toggle the approximation via $w_{i}$ in $(3.44 \mathrm{e})$.

Semi-JSNC method reduces computational complexity by decreasing number of variables and constraints. One of the most significant changes from previous problem formulations is that $\tau_{i}$ is not an optimization variable any more. Instead, the incentive offered to a user is determined based on (3.14). Moreover, there are $N+2$ binary variables for each user, instead of having $n m+2$ of them in (3.35). Since $\check{\Pi}\left(d_{i}\right)$ is univariate, $N$ can be much less than $n m$. Also, the number of equality constraints are reduced from 5 to 3 per user compared to JSNC. Run-time comparisons and numerical results of the presented SNC methods are discussed in the following section. 


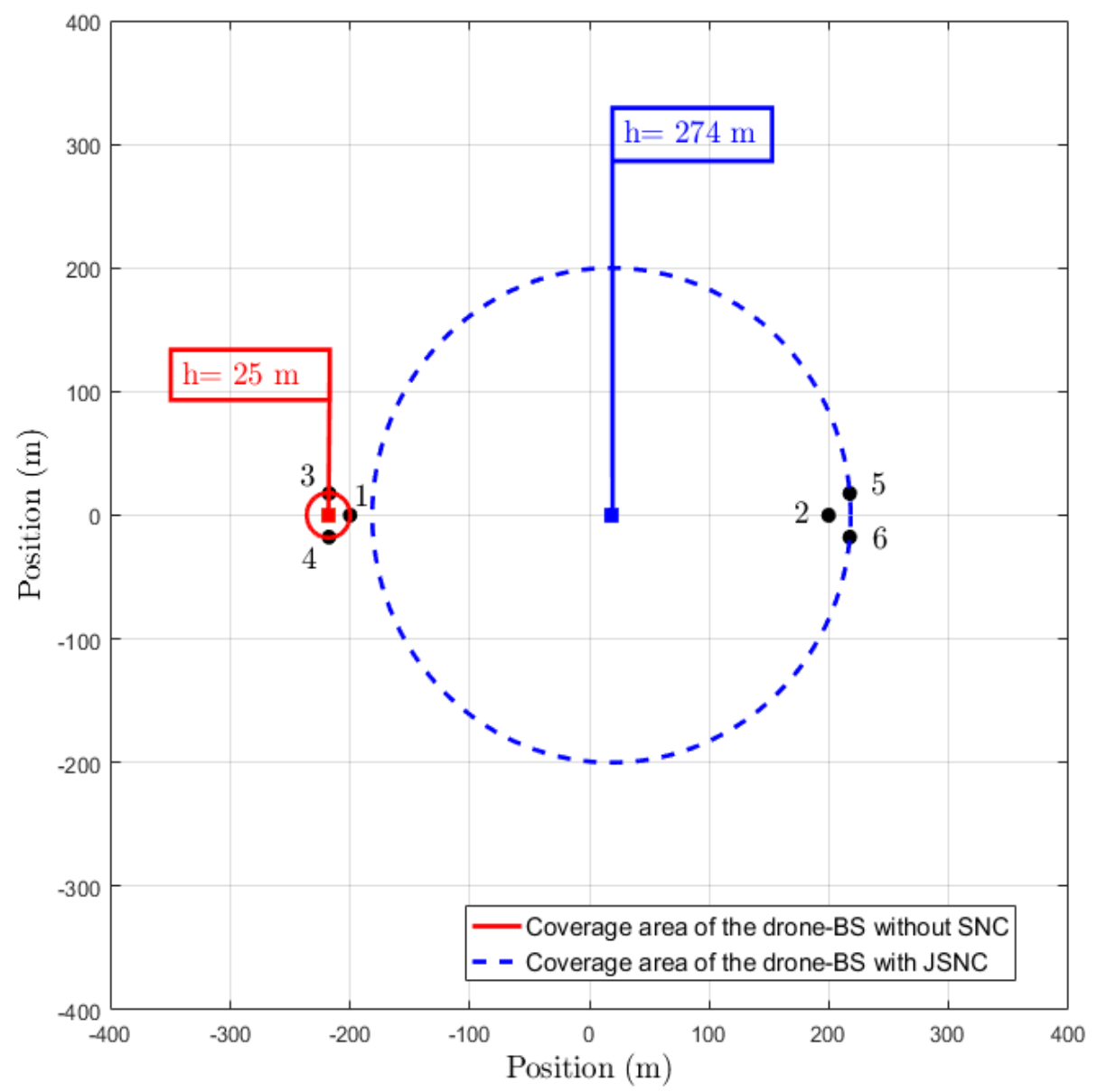

Figure 3.9: A simple example demonstrating the difference of placement and profit with and without SNC framework. Normalized profit is 3 without SNC, and 4.16 with JSNC.

\subsection{Simulations and Results}

We consider the dense urban environment whose parameters are provided in Table 2.5. For the simulations, we assume one drone-BS is to be positioned. We also assume that the drone-BS utilizes orthogonal resources to any existing terrestrial infrastructure. Additional simulation parameters are provided in Table 3.1. First, we would like to demonstrate the benefits of the proposed SNC framework utilizing drone-BSs and UIL by using a simple example. In Fig. 3.9, users 1 and 2 are $400 \mathrm{~m}$ apart. Users $3 \& 4$ and $5 \& 6$ are $25 \mathrm{~m}$ apart from users 1 and 2, respectively. The provided environment and QoS parameters in Table 3.1 yields a maximum coverage radius of $200 \mathrm{~m}$ for a drone-BS with fixed transmit 
Table 3.1: Simulation Parameters

\begin{tabular}{|c|c|}
\hline Parameter & Value \\
\hline$\left(x_{l}, x_{u}\right)$ & $(-700,700) \mathrm{m}$ \\
\hline$\left(y_{l}, y_{u}\right)$ & $(-700,700) \mathrm{m}$ \\
\hline$\gamma$ & $90 \mathrm{~dB}$ \\
\hline$f_{c}$ & $2.5 \mathrm{GHz}$ \\
\hline$d_{u}$ & $200 \mathrm{~m}$ \\
\hline $\mathrm{W}$ & $700 \mathrm{~m}$ \\
\hline$\Gamma^{*}$ & $209 \mathrm{~m}$ \\
\hline Monte Carlo Runs & 100 \\
\hline$\tau$ vertices, $\tilde{\tau}$ & $\{0.05,0.1,0.2,0.9\}$ \\
\hline$d$ vertices, $\tilde{d}$ & $\{5,10,20,40,200\}$ \\
\hline Number of breakpoints for Semi-JSNC, $N$ & 3 \\
\hline Number of users, $N_{u}$ & 15 \\
\hline
\end{tabular}

power. Therefore, the drone-BS cannot cover all the users while satisfying their QoS requirements. As seen in Fig. 3.9, the efficient drone-BS placement method described in Section 3.3.1 covers only one of the groups with the minimum required coverage area (red circle with dotted line), which is the optimal solution in this scenario without SNC. It provides a normalized profit of 3. On the other hand, the proposed JSNC method covers the group of users 2,5 , and 6 , and offers incentives to the remaining users (blue circle with dotted line). Note that, not only coverage radius is expanded to $200 \mathrm{~m}$, but also altitude, and horizontal location of the drone-BS is adjusted. Hence, JSNC provides total normalized profit of 4.16 , which is $39 \%$ more than the previous method. Although the SNC can be beneficial for future wireless networks, it can increase computational complexity for 3-D drone-BS placement. In order to address this issue, three methods are proposed in this study with varying computational complexities. The performance of these proposed methods is compared by randomly placing 15 users in $\mathcal{W}$ (Fig. 3.6) with parameters provided in Table 3.1. The cumulative distribution function (cdf) of the computation time of each method is given in Fig. 3.10. Note that, the computational complexity of the USNC is similar to that of the scheme without UIL, because once the drone-BS is placed, the optimal incentive to the users within the extended coverage area ( $\mathcal{D}$ in Fig. 3.6) is calculated simply by using 


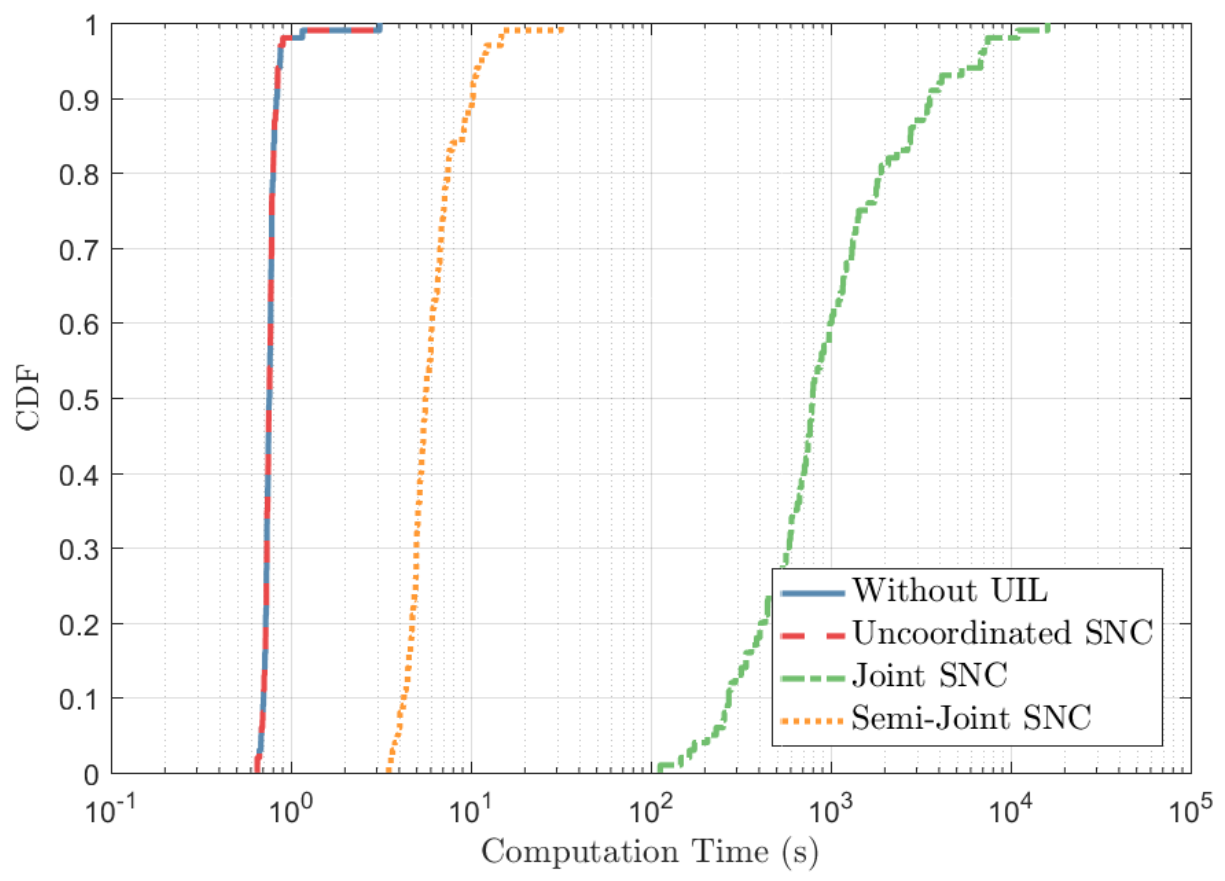

Figure 3.10: CDF of the computation time of the proposed methods.

(3.14). On the contrary, JSNC method, which considers the placement of the drone-BS jointly with the incentive amount to be offered to each user, has the highest computational complexity. Since the JSNC is a computationally costly method, semi-JSNC is proposed in Sec. 3.7. As shown in Fig. 3.10, semi-JSNC reduces computational time by more than 2 orders of magnitude. At the same time, the performance of the semi-JSNC method is very close to that of the JSNC as Fig. 3.11 demonstrates. Both JSNC and semi-JSNC outperform USNC in terms of normalized profit.

Finally, Fig. 3.12 shows the mean normalized profit with respect to the user density. The user density is calculated as

$$
\frac{N_{u}}{\pi \mathrm{W}^{2}} .
$$

In this experiment, $\mathrm{W}=700 \mathrm{~m}$ and a varying number of users from 10 to 27 are distributed randomly. For each number of users, 10 simulations are conducted, and the average of these simulations is calculated. Fig. 3.12 shows that semi-JSNC outperform USNC and the scheme without UIL, where the latter two provide very similar results. This indicates that USNC is not enough to attract enough number of users to provide a significant gain, and the situation does not improve with increasing density of users. On the other hand, gain 


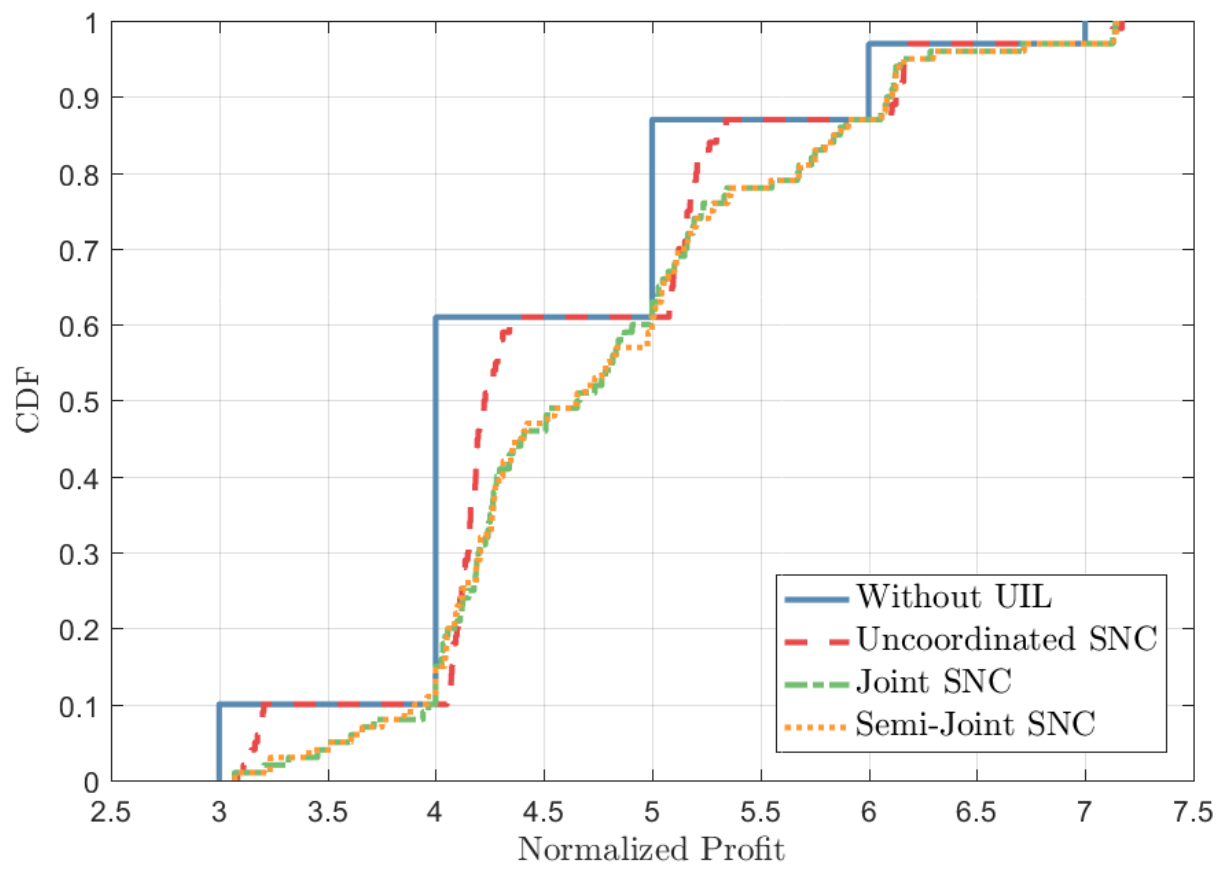

Figure 3.11: CDF of the profit obtained by the proposed methods.

from semi-JSNC increases with increasing user density, relative to the USNC gain. Note that, JSNC is not depicted in this figure, due to computational complexity.

\subsection{Summary and Discussions}

In this chapter, we proposed a novel method, SNC, based on exploiting the mobility of both the users and the drone-BSs. We first propose an uncoordinated SNC method, which is based on placing the drone-BS in 3-D space, and then offering the incentives to users. Although USNC is computationally efficient, the performance gain from considering these elements (drone-BS and UIL) separately provides a limited gain in terms of number of served users (added capacity to the system), because the drone-BS is not positioned by considering potential gain from UIL. In order to alleviate this issue, we proposed a joint-SNC method, which can be used to obtain the optimal incentives and 3-D coordinates simultaneously. However, the gain from UIL increases at the cost of computational complexity. Therefore, we propose a semi-joint SNC method, which is computationally more efficient 


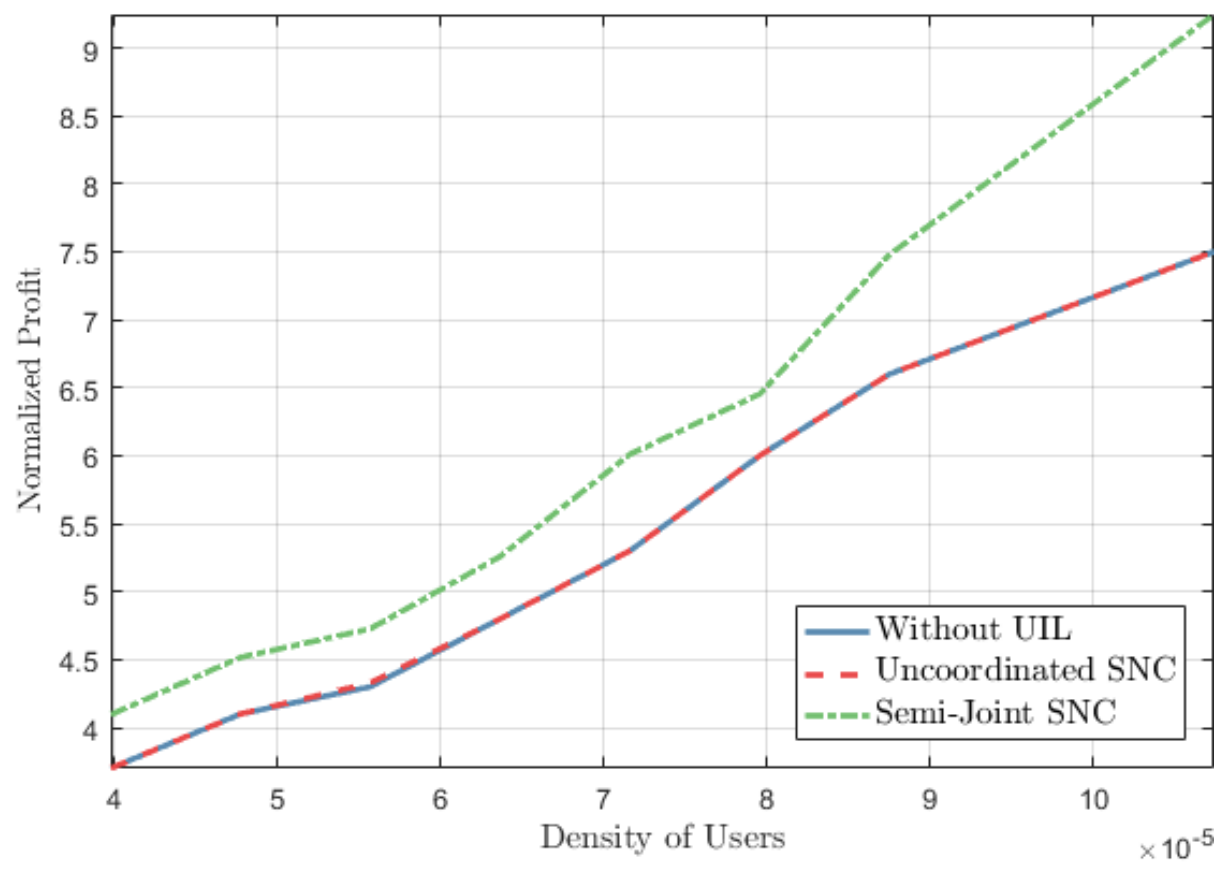

Figure 3.12: The comparison of the proposed methods based on mean unit profit versus the density of the users.

compared to joint-SNC, and provides similar results.

Once drone-BSs are efficiently utilized via SNC, their flexibility and customizable nature can make them suitable to enable agile networking, which is useful for many futuristic scenarios such as IoT, tactile internet, or green networking. Note that incentive methods are different than pricing, which focuses on QoS levels rather than user involvement [201]. However, especially when monetary incentives are considered, jointly determining pricing and incentive design can be beneficial to increase efficiency of wireless networks. In this thesis, the incentive design solely depends on the spatial location of the users. However, there can be many other dimensions to be considered, among which the QoS requirements stand out. Since the pricing mechanisms are good indicators of QoS levels, network congestion, and profitability, exploring their relation to incentive design can reveal beneficial frameworks for costly drone-BS operations. Finally, the placement and incentive options presented here are not necessarily ultimate placements and incentives, rather, they can serve as good a initialization for adaptive algorithms. Running such algorithms efficiently requires using capable data collection and processing methods, as well as successful integration of drone-BSs to the underlying terrestrial networks. These issues are discussed in the 
following chapter. 


\section{Chapter 4}

\section{Integration of Aerial Nodes}

In the previous chapters, we discussed that drones equipped with transceivers, i.e., drone base stations (drone-BSs) forming drone-cells, can help satisfy the demands of the future wireless networks [2, 31, 43, 62]. Moreover, they can utilize the latest radio access technologies (RATs), such as millimeter wave (mmWave), and free-space optical communication (FSO). In this chapter, we approach to the relationship between drones and wireless networks from a holistic, system-level perspective. Miscellaneous assets of drones and placement options provide opportunity to create multi-tier drone-cell networks to enhance connectivity whenever, wherever, and however needed. Therefore, the main advantage of drone-cells is the radical flexibility they create for wireless networks. On the other hand, wireless networks can provide reliable, secure and high-capacity services to drones as UEs, e.g., delivery drones. The phenomenon of providing ubiquitous connectivity to diversified user and device types is the key challenge for $5 \mathrm{G}$ and beyond-5G wireless networks. The Achilles' heel of the proposed technologies, such as decreasing cell size, cloud radio access networks (C-RAN), distributed antenna systems (DAS), and heterogeneous network (HetNet) deployments, is their rather rigid design based on long-term traffic behaviour [202]. In case of unexpected and temporary events creating hard-to-predict inhomogeneous traffic demand [130], such as natural disasters, traffic congestion, or concerts, wireless networks may need additional support to maintain ubiquitous connections. Drone-cells address this need by increasing relevance between the distributions of supply (BSs) and demand (user traffic). They can be used opportunistically to leverage the heterogeneity, i.e., by dynamically deploying BSs with different power levels and RATs. 
Although discussions on utilizing drone-cells in cellular networks have flourished recently $[31,35,103,203,204]$, the readiness of cellular networks to employ such dynamic nodes has not been discussed. For instance, drone-cells require seamless integration to the network during their activity and seamless disintegration when their service duration is over. This requires the capability of configuring the network efficiently, for which configuration and management flexibility, and self-organizing capabilities of the 3GPP Long-Term Evolution (LTE) networks may not be adequate. Hence, updating the network, such as adding new applications, tools, and technologies, is time and money consuming [205]. Also, massive amounts of granular information about users and networks must be continuously collected and analysed by intelligent algorithms. Collecting, storing, and processing big data is challenging for existing wireless networks [202]. Moreover, it is not yet clear how to balance centralized (e.g., mobile cloud) and distributed (e.g., mobile edge computing) paradigms [205].

Recent proposals for future wireless network architectures aim at creating a flexible network with improved agility and resilience. Cloud computing, SDN, and NFV have been proposed to relax the entrenched structure of the wireless networks, increase openness, ease configuration, and utilize cloud computing for storing and analysing big data. At the same time, these technologies may decouple the roles in the business model into infrastructure providers (InPs), mobile virtual network operators (MVNOs), and service providers (SPs) [206], which also changes the owners and sources of information.

In order to utilize drone-cells in future wireless networks, we propose a drone-cell management framework and discuss the related business and information models. The proposed framework relies on creating intelligence from big data in the cloud and re-configuring the network accordingly by SDN and NFV. After analyzing capabilities of the proposed drone-cell management framework, we survey recent 3GPP standardization studies for 5G to assess the readiness of $5 \mathrm{G}$ to support drones, either as UEs, or transceivers (BS, relay, distributed unit (DU)).

This chapter begins with an overview of drone-BS systems, and the opportunities and challenges associated with them. Then, we introduce the DMF and discuss its affect on business and information models of the wireless networks. We evaluate effectiveness of the DMF with a case study including 


\subsection{The New Frontier in RAN Heterogeneity: Multi-Tier Drone-Cells}

\subsubsection{Descriptions and Opportunities}

A drone-BS is a low-altitude ${ }^{1}$ unmanned aerial vehicle equipped with transceivers to assist the wireless networks [31], and drone-cell is the corresponding coverage area. Size of a drone-cell varies based on the drone-BS's altitude, location, transmission power, RATs, antenna directivity, type of drone, and the characteristics of the environment. Hence, multitier drone-cell networks can be constructed by utilizing several drone types, which is similar to the terrestrial HetNets with macro-, small-, femtocells, and relays. A multi-tier dronecell network architecture, assisting the terrestrial HetNets in several cases, is depicted in Fig. 4.1.

Drone-cells are useful in scenarios requiring agility and resiliency of wireless networks because they can prevent over-engineering. These type of scenarios can be categorized as temporary, unexpected, and critical, as shown in Table 4.1, where relevant test cases of the METIS ${ }^{2}$ project are listed [208]. Based on the scenario, the benefit to the network from a drone-cell varies. For instance, in traffic jam, stadium, and dense urban information society scenarios, a drone-cell can help prevent unexpected or temporary congestion in the network. Alternatively, drone-cells can improve resilience of wireless networks by providing additional coverage in case of a natural disaster, or by enabling teleprotection for the smart grid.

Critical scenarios have challenging demands, such as very high data rates, high reliability, or low energy consumption. Beyond the benefits to the network, providing connectivity in some of these scenarios is important to prevent serious losses, for example by saving lives in emergency communications, or increasing the lifetime of sensors and actuators at hard to reach areas. In case of emergency communications, and tele-control applications, drone-cells can enable high data rates and reliability, especially for situations in which the

\footnotetext{
${ }^{1}$ The classification of drones is a rather involved task due to their variety [207, Ch. 5]. However, in this context, the term "low-altitude" is used to differentiate drone-BSs from the high altitude platforms (HAPs) operating over $20 \mathrm{~km}$.

${ }^{2}$ Mobile and Wireless Communications Enablers for Twenty-twenty (2020) Information Society.
} 


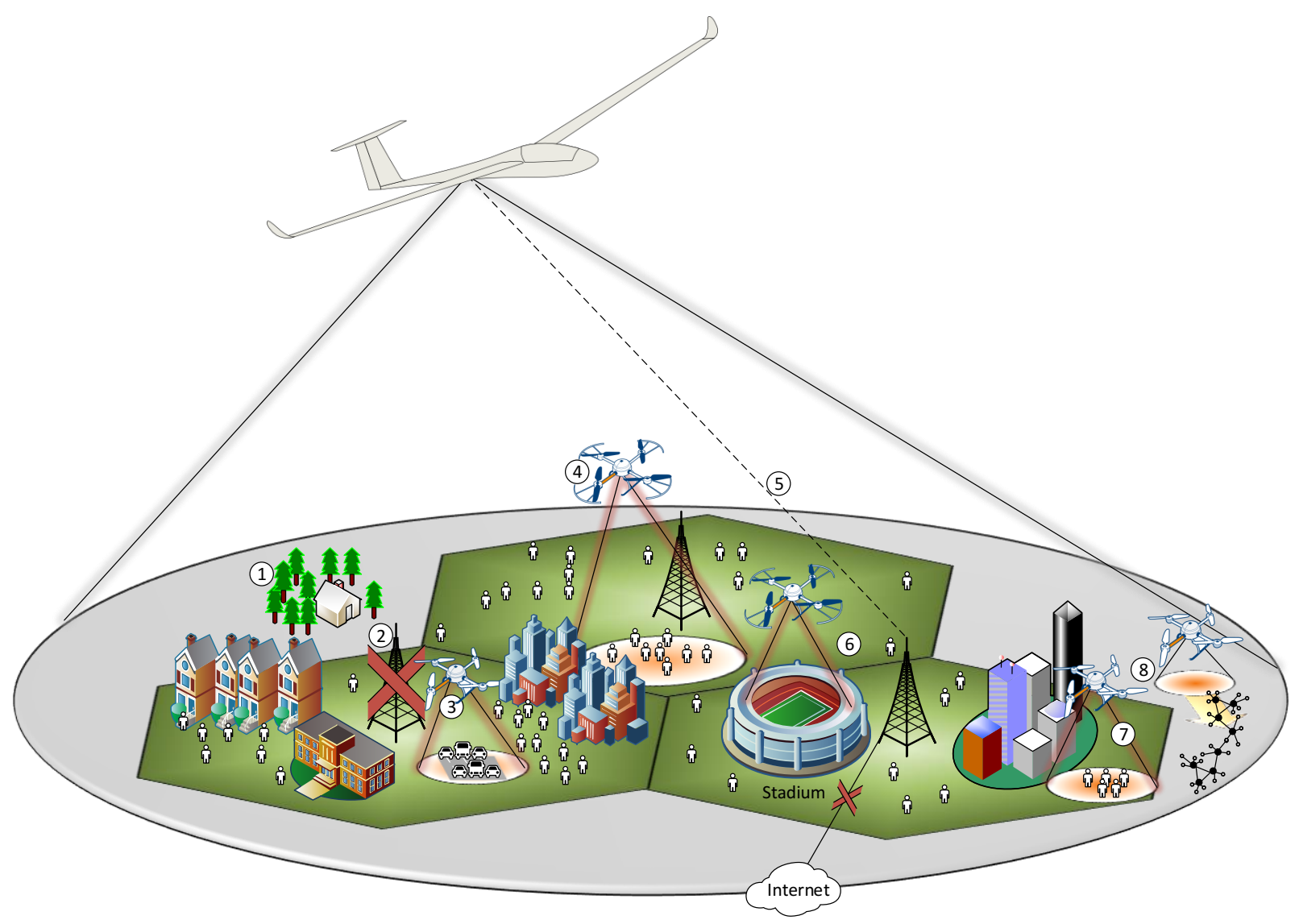

Figure 4.1: Multi-tier drone-cell networks can be used for many scenarios: (1) Providing service to rural areas (macro-drone-cell), (2) Deputizing for a malfunctioning BS (macro-drone-cell), (3) Serving users with high mobility (femto-drone-cell), (4) Assisting a macrocell in case of RAN congestion (pico-drone-cell), (5) Assisting a macrocell in case of core network congestion or malfunctioning (macro-drone-cell), (6) Providing additional resources for temporary events, e.g., concerts and sports events, (7) Providing coverage for temporary blind spots, and (8) Reducing energy dissipation of sensor networks by moving towards them (femtodrone-cell).

conventional modes of wireless access are either not present or difficult to establish. Mobility of drone-cells enables them to serve users with high mobility and data rate demand, e.g., for traffic efficiency and safety [208]. Alternatively, sensor-type devices requiring low energy consumption can benefit from drone-cells. Instead of forcing low-power devices to transmit to farther BSs, or deploying small cells densely, mobile sinks can be used. A 
Table 4.1: An example of categorization of test cases of METIS requiring agility and resilience: An event can fall under one category or multiple categories and each combination may require different solutions. For instance, connectivity requirements in case of an only temporary event (e.g., stadium) may be addressed by overengineering. Then, expenses of drone-BS operations may be compared to the expenses of over-engineering, including energy and maintenance costs. On the other hand, for both temporary and unexpected events, (e.g. traffic jam), drone-BSs may be utilized opportunistically. For temporary, unexpected and critical operations (e.g., emergency communications) drone-cells can provide much more than revenue, such as saving lives.

\begin{tabular}{|c|c|c|c|}
\hline Test Case & Temporary & Unexpected & Critical \\
\hline Stadium & $\mathrm{X}$ & & \\
\hline Teleprotection in smart grid & & $\mathrm{X}$ & \\
\hline Traffic jam & $\mathrm{X}$ & $\mathrm{X}$ & \\
\hline Blind spots & $\mathrm{X}$ & $\mathrm{X}$ & \\
\hline Open air festival & $\mathrm{X}$ & & \\
\hline Emergency communications & $\mathrm{X}$ & $\mathrm{X}$ & $\mathrm{X}$ \\
\hline Traffic efficiency and safety & & & $\mathrm{X}$ \\
\hline Dense urban information society & $\mathrm{X}$ & & \\
\hline Massive deployment of sensor-type devices & $\mathrm{X}$ & $\mathrm{X}$ & $\mathrm{X}$ \\
\hline
\end{tabular}

drone-cell can move towards clusters of devices and provide low-power communication due to its proximity and potential line-of-sight (LoS) connectivity. In particular, when unexpected events trigger massive sensor activity, drone-cells can reduce the overall stress on the network and increase the life-time of sensors. Note that the critical scenarios, in which the conventional wireless access options are not feasible, may render them as the first applications of drone-cells in providing (almost) carrier-grade service.

Although the flexibility of drone-cells allows utilizing them in versatile scenarios, it creates significant design, operation, and management challenges, which are discussed next. 


\subsubsection{Challenges of Drone-cells}

\section{Efficient design}

Drones have been utilized for military, surveillance and reconnaissance applications for long time. However, their usage in cellular communications as drone-BSs is a novel concept under investigation. For instance, a preliminary implementation of an LTE eNodeBbased drone operation was presented in [203], where a remote radio head (RRH) was deployed on an off-the-shelf helikite. The helikite was tethered to a truck carrying the baseband unit (BBU), and optical fiber is used for the fronthaul. This tethered helikite design is due to the non-existence of drones that are specifically designed to operate as drone-BSs. Drones are generally designed for their task, which is the reason for their great variety [207, Ch. 5].

Drone-BSs would have unique requirements that can benefit from special-purpose designs, such as long-time hovering, long endurance, robustness against turbulence, minimum wing-span allowing MIMO, and provision of energy for transmission (in addition to flying). For instance, a hybrid-drone can be designed with vertical take-off capability of rotorcrafts and with collapsible wings (equipped with MIMO antenna elements and solar panels for energy harvesting), which can be unfolded for efficient gliding.

Designing the payload of drone-BSs is as important as determining their mechanics, e.g., size, aerodynamics, and maximum take-off weight [207, Ch. 9]. For efficient usage of the limited volume, weight, and energy of drone-BSs, the payload can vary according to the scenario. Several possible drone-cell configurations are listed below:

- Drone-relay ("Drolay”): Compared to small- or macro-BSs, relays require less processing power, because their RRH may be relatively simple and they may not require an on-board BBU. Hence, they operate with light payloads, i.e. additional equipments to the ones required for drone's own operation, and potentially consume less power. The size and weight of RAN nodes may not be critical for terrestrial HetNets, however a lighter payload improves endurance and decreases CAPEX and OPEX significantly in drone-cell operations.

- Small-drone-BS: They resemble terrestrial small-BSs with wireless backhaul. If a reliable wireless fronthaul can be maintained despite the mobility of drone-BSs, its 
advantage is twofold: First, it alleviates the weight and processing power required for an on-board BBU. Second, if combined with C-RAN, it can allow cooperation. C-RAN is useful particularly for dense HetNets [202], or when a fleet of drone-BSs are deployed. Scenarios (3), (4, (7), and (8) in Fig. 4.1 exemplify potential usage.

- Macro-drone-BS: They resemble terrestrial macro-BSs with wireless backhaul. They can be deployed for longer endurance, broader coverage, or increased reliability of the network, e.g., (1), (5) and (6) (Fig. 4.1). BBU can be included if a reliable wireless backhaul exists. Since coverage is strongly related to altitude and power, macro-drone-BSs may have a larger size, which allows more payload, e.g. mediumaltitude long-endurance drones [207, Ch. 113].

In addition to the discussion above, efficient drone-cell design can be enhanced by advancements on low-cost and light-weight energy harvesting, high-efficiency power amplifiers, beyond visual LOS operations, and alternative fuels, to name a few.

\section{Backhaul/fronthaul connection}

In terrestrial networks, wireless backhaul/fronthaul is considered when fiber connectivity is unaffordable, e.g., dense HetNets or rural BSs. However, it is inevitable for multi-tier drone-cell networks. FSO and mmWave are promising for their high-rate and low spectrum cost. However, their reliability and coverage are limited, especially for inclement weather conditions [209,210]. Although mobility of drone-cells help maintain LOS, it necessitates robustness against rapid channel variations.

\section{Placement}

Terrestrial BSs are deployed based on long-term traffic behaviour and over-engineering when necessary. However, drone-cells require quick and efficient placement. Therefore, it is of critical importance to determine the parameters affecting a drone-cell's performance, such as its altitude, location, and trajectory based on the network demands [31, 32, 35, 43,51]. For instance, if a drone-cell is utilized to release congestion in RAN within a 
congested cell, the target benefit is to offload as many users as needed to the drone-cell [31]. Particularly, if the congestion is at the cell edge, the drone-cell can be placed right on top of the users there. On the other hand, if the congestion is at the backhaul, some of the most popular contents can be cached in a drone-cell for content-centric placement (Sec. 4.2.2). Moreover, placement of multi-tier drone-cell networks requires integrated evaluation of many other challenges.

\subsubsection{Challenges of Multi-tier Drone-cell Networks}

There are additional challenges of multi-tier drone-cell networks. Although these challenges are similar to those of terrestrial HetNets, the particular details related to drone-cells are discussed here.

- Physical layer signal processing: The link between the drone-cell and terrestrial nodes, i.e., air-to-ground links, have different characteristics than terrestrial channels $[10,20,27,29]$. However, the research on air-to-ground links is not mature and the proposed channel models vary depending on factors such as temperature, wind, foliage, near-sea environments, urban environments, and the aircraft used for measurement campaigns, to name a few. For instance, higher ground speed causes rapid variation of spatial diversity; users at different locations with respect to the drone-BS can have different channel characteristics simultaneously [20]. Therefore, designing robust signaling mechanisms with strict energy constraints of drone-BSs is challenging.

- Interference dynamics: Drone-cells in proximity can suffer from co-channel interference for their air-to-ground links, and backhaul/fronthaul. Moreover, a dronecell's mobility creates Doppler shift, which causes severe inter-carrier interference for RATs at high frequencies (e.g., mmWave). In HetNets, interference of terrestrial and air-to-ground-channels can decrease capacity. Therefore, advanced interference management schemes, which consider the characteristics of air-to-ground links and mobility of drone-cells, are required.

- Cooperation among drone-cells: The dynamic nature of multi-tier drone-cell networks requires cooperation among drone-cells for efficiency in radio resource management. In addition to that, drone-cells can cooperate to adapt to the mobility of 
the users to decrease handover, optimize power and resource allocations, and avoid collisions.

- Infrastructure decision and planning: The number and assets of drone-cells (e.g., access technology, memory, and speed) to be utilized for a multi-tier drone-cell network depend on circumstances, such as inclement weather conditions, size of the area to be served, type of service (e.g., virtual reality, internet-of-things), target benefit of the network (e.g., congestion release, resilience, low-latency), or service duration. Also, utilizing drone-cells with different access technologies can reduce interference, and increase capacity of multi-tier drone-cell networks, e.g., utilizing a macro-drone-cell with RF and small-drone-cells with mmWave to prevent frequency re-use. Hence, InPs must have a fleet which can respond to possible scenarios. To optimize the fleet and construct an efficient network, information sharing among all parties of the network, i.e., InPs, MVNOs and SPs, is required.

Cost, lack of regulations, security, and airworthiness are among other challenges of drones. The vital point of the matter is considering the effects of utilizing drones in highly sophisticated cellular communication networks, rather than using them for stand-alone applications, e.g., aerial photography or inspection. Therefore, drone-cells require an equivalently sophisticated management system, which is discussed next.

\subsection{The Drone-cell Management Framework}

A drone-cell is not a one-size-fits-all solution, instead, it is tailored based on the target benefit. Along with the management of individual drone-cells, multi-tier drone-cell networks require active organization and monitoring, e.g., for nodes changing location or cells becoming congested. Three capabilities are required to integrate drone-cells with already sophisticated cellular networks:

- Global information: The information gathered by BSs alone may be inadequate to generate intelligence for managing drone-cells. Global information, including location, type, and habits of the users, functionality of the BSs, and the contents to deliver must be stored and analyzed centrally. Big data and cloud computing can be effective solutions for that purpose. 
- Programmability: Both drone-cells and network tools need to be programmed based on the network updates. Moreover, sharing the resources made available by a drone-cell can reduce the CAPEX and OPEX. NFV can provide these capabilities to the wireless networks.

- Control: Wireless networks must be configured efficiently for seamless integration/disintegration of drone-cells, such as changing protocols and creating new paths. SDN can be useful to update the network automatically via a software-based control plane.

The current LTE architecture does not embody all of these abilities, but cloud, SDN, and NFV technologies can enable a more capable wireless communication system [202].

\subsubsection{Enabling Technologies for DMF}

In this subsection, we briefly explain the technologies that increase capabilities of wireless networks and the interactions that are required to efficiently manage drone-cell-assisted wireless communications.

\section{Cloud and Big Data}

There are many ways to approach the problem of collecting and processing sufficient data (Table 4.2) in a timely manner for efficiently utilizing drone-cells. A cloud for dronecells, consisting of computing power and data storage (Fig. 4.2), combined with big data analysis tools, can provide efficient and economic use of centralized resources for networkwide monitoring and decision making [205,211]. If drone-cells are owned by a traditional mobile network operator (MNO) (Fig. 4.2), the cloud is merely the data center of the MNO (similar to a private cloud), where the MNO as an administrator can choose to share its knowledge with some other players or use it for its own business purposes. Alternatively, if the drone-BSs are provided by an InP, the InP can use the cloud to collect information from MVNOs and SPs (Fig. 4.2) and Table 4.2). In this case, it is particularly important to guarantee security, latency, and privacy. Benefit of the cloud can be better exploited 
with a programmable (softwarized) network allowing dynamic updates based on big data processing, for which NFV and SDN can be enabling technologies.

\section{Network Functions Virtualization}

NFV alleviates the need for deploying specific network devices (such as packet and serving gateways, deep packet inspection modules, and firewalls) for the integration of dronecells [205]. By virtualizing the above-network functions on general purpose servers, standard storage devices, and switches, NFV allows a programmable network structure, which is particularly useful for drone-cells requiring seamless integration to the existing network (4) in Fig. 2). Furthermore, virtualization of drone-cells as shared resources among $\mathrm{M}(\mathrm{V})$ NOs can decrease OPEX for each party (Section 4.2.2) [206]. However, the control and interconnection of VNFs becomes complicated, for which SDN can be useful [205].

\section{Software Defined Networking}

By isolating the control and data planes of network devices, SDN provides centralized control, global view of the network, easy reconfiguration, and orchestration of virtual network functions (VNFs) via flow-based networking (4 in Fig. 2). Specifically for cellular networks, a centralized SDN controller can enable efficient radio resource and mobility management [205], which is particularly important to exploit drone-cells. For instance, SDN-based load balancing proposed in [205] can be useful for multi-tier drone-cell networks, such that the load of each drone-BS and terrestrial-BS is optimized precisely. An SDN controller can update routing such that the burst of traffic from the drone-cells is carried through the network without any bottlenecks [211]. Similarly, in case of a natural disaster that causes the network to partially malfunction, network health information in the cloud can be utilized via SDN to route the traffic of drone-cells through undamaged parts of the network. Because SDN allows updating switches simultaneously (e.g., for new forwarding rules), it allows faster switching between RATs [212], which eases utilizing different RATs in multi-tier drone-cell networks. Furthermore, the architecture based on hierarchical SDN controllers for unified handoff and routing proposed in [212] can allow granular management of flows through drone-cells. For instance, the handoff strategy can be changed to a more complex proactive handoff for decreasing the latency of flows from 
drone-cells. Alternatively, DMF may collaborate with the mobility management entities for

efficiency, e.g., a drone-cell can follow high-mobility users on a highway (3) in Fig. 4.1) to reduce handover. For further exploitation for the new degree-of-freedom introduced by the mobility of the drone-cells, the footprint of the drone-cells can be adjusted to optimize paging and polling, and location management parameters can be updated dynamically via the unified protocols of SDN.

\section{Business and Information Models of DMF}

In traditional cellular networks, an MNO owns almost the entire cellular network, such as BSs and core network, and sharing among MNOs is limited. However, future cellular networks may be partitioned between InPs, MVNOs and SPs [206]. For instance, high sophistication of drone operations may result in the drone-cell operator becoming a separate business entity, such as a drone-InP.

Fig. 4.2 represents a DMF with potential business and information models, and shows what is owned by these parties, and what information flows from them to the cloud. According to the model, all physical resources of the cellular network, including drone-cells, BSs, spectrum, and core network, are owned by InPs. The MVNO is responsible for operating the virtual network efficiently such that the services of the SP are delivered to the users successfully. Note that, in this model, perfect isolation and slicing is assumed such that an MVNO has a complete virtual cellular network [206].

Compared to the traditional cellular networks, more granular data is available, but it is distributed unless collected in a cloud. A brief list of information, which can be critical for the operation of the DMF, is provided in Table 4.2 along with its type, source, and usage [205]. The results of the processing are then used to orchestrate SDN and NFV for the purpose of integrating drone-cells into the networks. This mechanism is demonstrated in Section 4.2.2.

Note that such isolated business roles may not be realistic for the near future. Instead, the role of an MNO may get partitioned into three actors, namely, InP, MVNO, and SP. Since it will mature in the long run, this partitioning should not be considered as siloing, but rather specialization. Accordingly, unique pricing strategies and QoS monitoring requirements will likely appear for drone-cell operations. Although complex and expensive, 


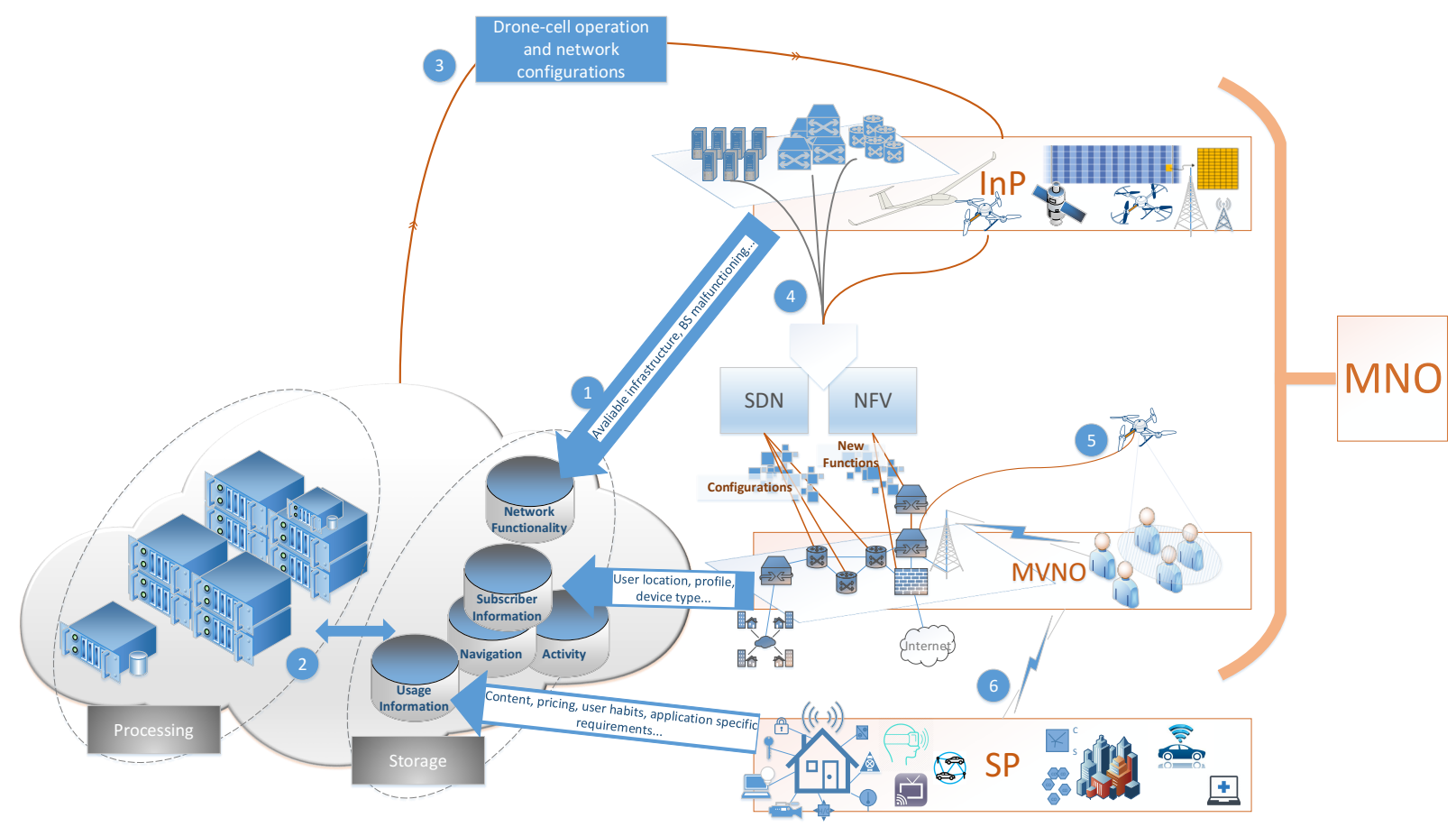

Figure 4.2: DMF mechanism and potential business and information model demonstrating partitioning of the traditional MNO into InP (cloud, server, drone-BS etc.) and MVNO: (1) Collect and store global data; (2) Process data for network monitoring and creating intelligence; (3) Provide guidance for drone-cell's operation (placement, content to be loaded, access technology, service duration, coverage area, moving patterns); (4) Re-configure the virtual network of MVNO for drone-cell integration by SDN and NFV technologies, e.g., introduce another gateway to handle busy traffic and create new paths among the new and existing functions; (5) Drone-cell assists the network; (6) SP can continue delivering services successfully.

drone-cell operations can increase revenues in several ways, such as enabling a leaner terrestrial network, service to high-priority users (e.g., for public safety), and continuity of challenging services even in cases of unpredictable high density traffic in areas with relatively insufficient infrastructure (Section 4.1.1). 
Table 4.2: Various information that can be gathered in the cloud.

\begin{tabular}{|c|c|c|c|}
\hline Information & Type & Source & Use \\
\hline International Mobile Subscriber Identity (IMSI) & User & MNO & True identity of the user \\
\hline User profile information & User & MVNO & Subscription type, activities \\
\hline User's location & Network & MVNO & Location \\
\hline Device type & Network & MVNO & Location, resource allocation provisioning, etc. \\
\hline Functionality of the nodes & Network & InP & Location, coverage extension, energy saving, etc. \\
\hline User's activity and navigation & Network & MVNO & Placement, consumption, lifestyle, etc. \\
\hline Content & Usage & SP & Centers of interest, preferences, pricing, content delivery, etc. \\
\hline Long-term historic data & Usage & SP & Content delivery, pricing, etc. \\
\hline
\end{tabular}

\section{Challenges for DMF Implementation}

Network management required for DMF involves the challenges of NFV and SDN. Slicing of drone-cells, isolation of the traffic of different MVNOs, migration of virtual network functions, virtual resource management, and scheduling can be listed among the major challenges related to NFV [206]. Regarding the SDN in DMF, the main challenges are providing a global view to the SDN controller, i.e., scalability, efficiency in programming new paths, and communicating with different virtual network entities and application interfaces [213]. Especially, latency as a performance indicator is critical for drone-cells. The flow- and cloud-based networking are promising approaches to overcome these challenges [205, 211-213].

Flow-based networking requires advancements, such as developing new routing protocols, interfaces, and applications. The major difficulties associated with the cloud are centralizing the distributed data, providing security, determining the level of sharing while satisfying the regulations, and providing the power required for processing massive amounts of data [202,211]. In this sense, real-time collection and processing of the data required to manage a drone's operation (e.g. tackling turbulence, avoiding collisions, tracking user mobility) is infeasible. Therefore, DMF is unlikely to alleviate the need for drones with high levels of autonomy [207, Ch. 70], but DMF can provide guidelines, as demonstrated in the following section. 


\subsubsection{A Case Study: 3-D Placement of a Drone-cell via DMF}

Efficient placement is a critical and challenging issue for drone-cells. In this section, we propose an objective for DMF, meeting various demands simultaneously. Then, we numerically illustrate the benefit of using DMF by comparing the results with the efficient 3-D placement ${ }^{3}$ method proposed in Section 2.1, and show that DMF can split costs among MVNOs without detracting from the network benefit in a multi-tenancy model.

Let us consider that a drone-cell, managed via DMF, is used to assist a terrestrial HetNet with the following considerations:

- Congestion release in RAN: A set of users, $\mathbb{U}$, cannot be served by the BS because of the congestion. The objective is to serve as many users from the set $\mathbb{U}$ as possible by the drone-cell. Let $u_{i}$ denote a binary variable indicating whether the $i^{\text {th }}$ user in $\mathbb{U}$ is served by a drone-cell with orthogonal resources. Note that $\mathbb{U}$ is determined by MVNOs based on connection characteristics of each user [205] (Table 4.2).

- Multi-tenancy: An InP owns the drone-cell and sends it to the congested macrocell according to the intelligence provided by the cloud (Fig. 2). This network structure allows sharing the drone-cell's resources, if desired, to maximize the revenue and reduce the OPEX. Assuming all users provide the same revenue (as in [31]), the number of users associated with an MVNO and served by the drone-cell can be a measure of the revenue provided to that MVNO. Hence, the objective becomes maximizing the number of served users, as well as forcing the drone-cell to serve the target number of users of each MVNO. Then, if the total number of MVNOs in the macrocell is $J$, a $\mathbf{J} \times 1$ vector $\mathbf{v}$ can be calculated, such that its $j^{\text {th }}$ element, $v_{j}$, denotes the ideal number of $\mathrm{MVNO}_{\mathrm{j}}$ 's users to be served by the drone-cell. Also, the cloud must store the vector $\mathbf{u}$ containing $u_{i}$, which indicate whether user $i$ is served by the drone-BS, and the matrix $\mathbf{S}$, which denotes the user-MVNO associations. $S(i, j) \in\{0,1\}$ indicates whether user $i$ belongs to MVNO $j$, which can be known from the subscriber information in the cloud (Table 4.2). Note that $\mathbf{v}$ is derived by cloud computing, based on several factors, such as agreements between the InP and MVNOs, pricing, user mobility, requested contents, and the scenario (Table 4.2, Fig. 4.2).

\footnotetext{
33-D placement concept is introduced in [31] because the probability of having LOS connection increases with increasing altitude, and, at the same time, path loss increases due to increased distance. Therefore, an optimum altitude is sought after, as well as an optimal area to cover in the horizontal domain.
} 
- Green wireless communications: $\boldsymbol{\Lambda}$ represents the energy cost of users. Hence, the drone-cell can be placed close to the energy critical users, such as sensor-type devices, or those at the blind spots (7 in Fig. 4.1). Device-type information is collected by the MVNO (Table 4.2).

- Content-centric placement/Congestion release at the backhaul: $\kappa_{i}$ indicates if the user $i$ requests a popular and costly content (e.g., in terms of bandwidth or price), $\kappa$, which is cached in the drone-cell. Hence, the placement can be adjusted according to the content requirements of the users. Note that decisions about which contents to be delivered depends on the short- and long-term data collected by SPs on usage, user habits, and so on (Table 4.2 and Fig. 4.2).

Then, a comprehensive placement problem can be written as

$$
\begin{array}{ll}
\max _{\mathbf{p},\left\{u_{i}\right\}} & \omega_{1} \sum_{i \in U} u_{i}+\omega_{2}\|\mathbf{S u}-\mathbf{v}\|+\omega_{3}\|\mathbf{S u}-\boldsymbol{\Lambda}\|+\omega_{4} u_{i} \kappa_{i} \\
\text { s.t. } & Q\left(\mathbf{p}, u_{i}\right) \geq q_{i}, \quad \forall i=1, \ldots,|\mathbb{U}|, \\
& \mathbf{p} \in \mathbb{P}, \\
& \sum_{i \in \mathbb{U}} u_{i} R_{i} \leq C, \quad \forall i=1, \ldots,|\mathbb{U}|, \\
& u_{i} \in\{0,1\}, \quad \forall i=1, \ldots,|\mathbb{U}|,
\end{array}
$$

where $|\cdot|$, and $\|\cdot\|$ represent the cardinality of a set and vector norm operation, respectively; $\omega$ represents the weight of each benefit; $\mathbf{p}$ denotes the location of the drone-cell in 3-D space; $Q\left(\mathbf{p}, u_{i}\right), q_{i}$, and $R_{i}$ denote the QoS delivered to the $i^{t h}$ user from the drone-cell at location $\mathbf{p}$, the minimum tolerable service quality e.g., signal-to-noise ratio (SNR), and the required resources to serve the $i^{\text {th }}$ user (e.g., bandwidth), respectively. $C$ represents the capacity of the drone-cell and $\mathbb{P}$ denotes the set of allowable locations for placing the drone-cell, such as the allowed distance from the buildings according to regulations, or the positions with LOS links to the backhaul/fronhaul node. Note that the weights among the benefits, $\omega_{i}$, can be determined based on their importance to the owner of the drone-cells. Similarly, determining $\omega_{i}, \mathbf{v}$, and $\kappa_{i}$, based on their importance to the owner of the drone-cells, are interesting problems themselves.

The generic problem in (4.1) is mathematically formulated in [31] by assuming $\omega_{1}=1$, 
and the rest of the weights are 0. Air-to-ground channel model in [27] relates the size of a drone-cell to the altitude of the drone-BS. Therefore, both horizontal and vertical coordinates of a drone-BS must be determined simultaneously. Hence, an efficient 3-D placement algorithm is proposed to find the optimal altitude, as well as an optimal area to cover in the horizontal domain as in Section 2.1. As a result, maximum number of users are covered with a minimum required area. In this part of the study, 3-D placement of a drone-cell is improved to allow and regulate multi-tenancy by DMF.

If single-tenancy is considered, only users subscribed to $\mathrm{MVNO}_{1}$ are served by the drone-BS (as in Chapters 2 and 3). If multi-tenancy is allowed, users of both $\mathrm{MVNO}_{1}$ and $\mathrm{MVNO}_{2}$ can be served. In this case, it is important to regulate the amount of service delivered to each MVNO, so that their agreements with the InP is not violated. Therefore, we assume that $\omega_{1}=\omega_{2}=1$, and $\omega_{3}=\omega_{4}=0$ corresponds to the case of regulated multitenancy with DMF, and only $\omega_{1}=1$ corresponds to either single-tenancy, or multi-tenancy without DMF.

For a numerical comparison, assume that there are 30 users that cannot be served by the terrestrial HetNet. They are distributed uniformly and arbitrarily subscribed to one of the two available MVNOs. The QoS requirement for all users is the minimum SNR (100 dB maximum tolerable path loss). Also, MVNOs are identical, e.g. in terms of their agreements with InP, user priorities, and QoS requirements. Therefore, $v_{1}=v_{2}=15$, which is in favour of providing an equal amount of service to each MVNO. Hence, they can share the cost of the drone-cell equivalently.

Fig. 4.3 shows how the placement of a drone-cell changes with respect to policies, namely, single-tenancy and multi-tenancy with and without DMF. The circular areas indicate the coverage of the drone-cell, and enclosed users are served by the drone-BS, i.e., their QoS requirements are satisfied. In case of single-tenancy, the coverage area is shown with the red circle, and a total of 6 users of $\mathrm{MVNO}_{1}$ (users shown with blue squares 2, 4, 5, $8,10,23)$ are covered. Note that, users 9 and 16 belonging to $\mathrm{MVNO}_{2}$ are not served in this case. On the other hand, 10 users are enclosed in both green and orange drone-cells with multi-tenancy. In the orange drone-cell representing the placement without DMF, 6 users belong to $\mathrm{MVNO}_{2}$ and 4 users belong to $\mathrm{MVNO}_{2}$. Hence, the resources of the drone-BS are not equally distributed as suggested by the cloud. That may reduce the benefit of the 


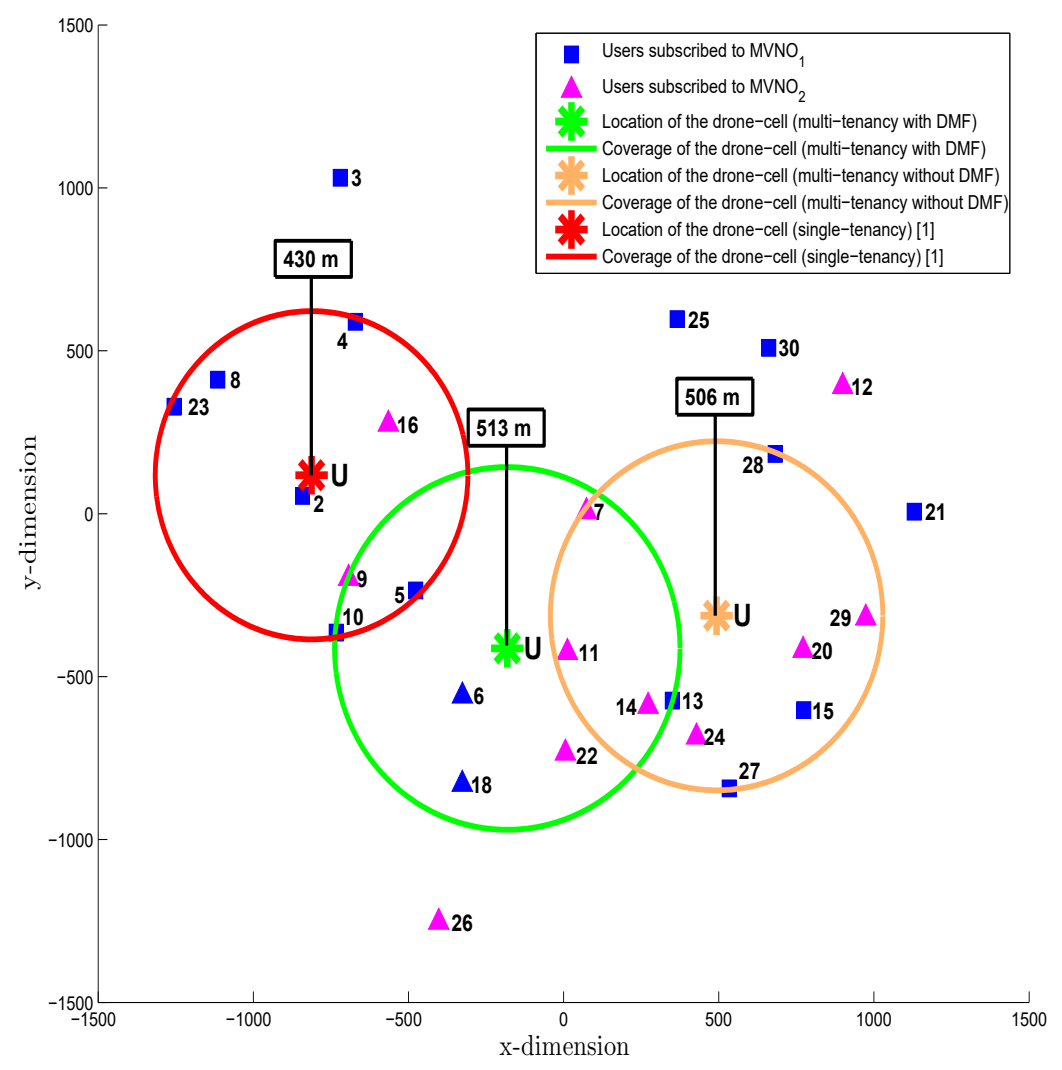

Figure 4.3: Effect of different policies on 3-D placement of a drone-BS.

network, e.g., $\mathrm{MVNO}_{2}$ may reject the drone-BS's services. However, when DMF is considered, 5 users of each MVNO are served in the green drone-cell. At the same time, there is no compromise in the network's benefit, since the total number of served users remains the same in both multi-tenancy scenarios. Note that not only single- or multi-tenancy (red vs. green and orange circles), but also regulating the service among MVNOs changes the placement (green vs. orange circles).

In order to clarify the advantage of DMF, we compared single-tenancy [31] with multitenancy regulated by DMF. In Fig. 4.4, 30 idle users in four different environments [28] are randomly distributed, and the results of 100 Monte Carlo simulations are averaged. It shows that $\mathrm{MVNO}_{1}$ serves almost the same number of users (1-2 users less in each case) when it shares the drone-cell with $\mathrm{MVNO}_{2}$. In turn, the drone-cell's cost can be reduced by a factor of two. Moreover, the total number of served users increases (approximately 1.5 


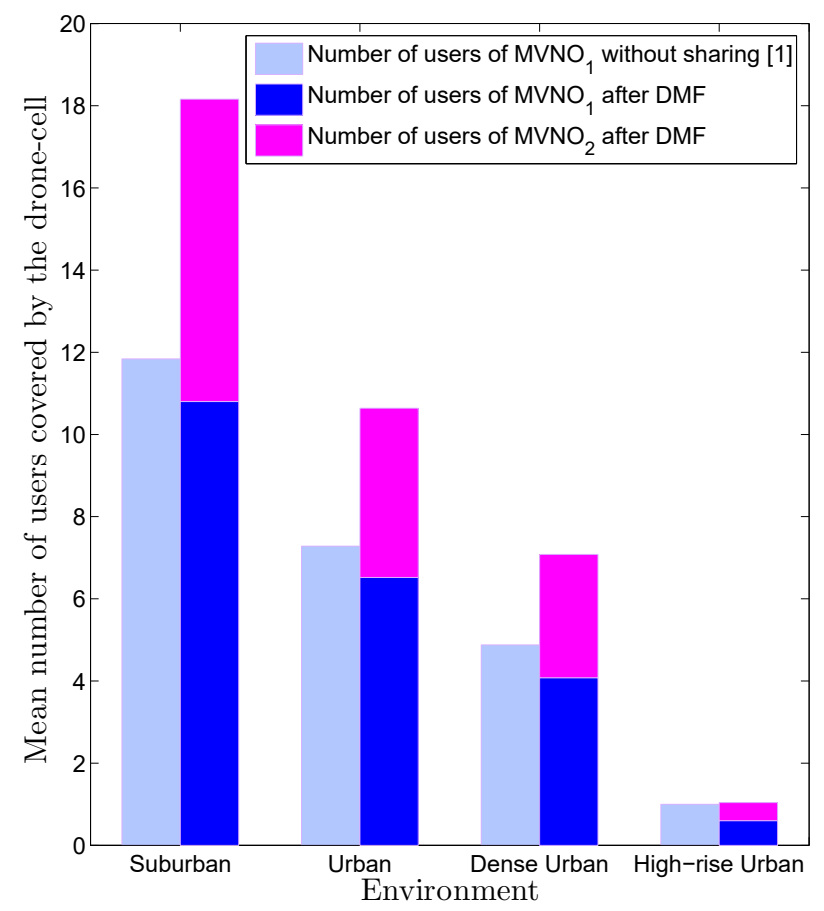

Figure 4.4: Mean number of users covered by the drone-cell with 3-D placement in different environments.

times), which means that more congestion is released from the network.

Although implicit here, the number of covered users can also indicate the amount of injected capacity, enhanced coverage, and reduced re-transmission time in a congested scenario. Moreover, we have demonstrated the 3-D placement of one drone-cell, although, multi-tier drone-cell networks require additional considerations, such as inter-cell interference, cell density, cooperation of drone-cells, and green networking. Therefore, collecting data to define the problem in (4.1), and then analyzing it efficiently requires a holistic and centralized cellular network, rather than the existing distributed one. The better drone-cells are managed, the more the advantages of their flexibility can be exploited. 


\subsection{Drone-BS Integration with Virtual Wireless Access}

Wireless access virtualization has been considered as one of the key methods to exploit densification, multiple radio access technologies, and MIMO capabilities of future wireless networks [214,215]. In this section, we investigate the synergy between the flexibility of virtual wireless access (VWA) techniques and integration needs of drone-BSs.

The VWA framework under consideration incorporates user-centric transmit point (TP) clusters and provides macro-diversity gain. Although this VWA framework is similar to user-centric no-cell (UCNC) that is widely used in the literature [216], the VWA framework does not have overlapping clusters. The core idea of UCNC is exchanging the classical wireless "cell" with virtual cells formed around users, such that users do not experience edge conditions. That is achieved by forming serving TP groups for each user based on a specific criterion, such as reference signal received power [215,217]. The formation of virtual cells can be achieved via several methods, such as beamforming and coordinated scheduling. The study in [218] proposes a user-plane virtualization method, which takes advantage of cloud-RAN and device-to-device communications for a given TP set. Similar to the study in [215], [214] allows users to request service or interference nulling from TPs causing strong and moderate interference, respectively. That method requires careful setting of parameters and does not consider the joint effect of all user choices.

Integration of a drone-BS into the VWA framework is shown in Fig. 4.5, where 3 TPs and a drone-BS are serving users. Two TP groups, i.e., virtual cells, are formed as depicted with dotted lines. These groups are determined by considering user distribution for a snapshot, which may include several scheduling intervals. In Fig. 4.5, TP1 and TP2 have the highest number of users experiencing edge conditions, as well as the drone-BS and TP3. Moreover, in Fig. 4.5 drone-BS is positioned to cover the maximum number of users with minimum area [31]. The depicted scenario here is an ideal case to benefit from the drone-BS, because those users are clustered at the edge of one of the TPs (TP3). In this framework, it is important to both place the drone-BS in 3D space and determine best TP groupings for the benefit of the network. However, in the beginning of a snapshot, neither the position of the drone-BS, nor the TP groups are known. Therefore, we propose several strategies to find optimal solutions for this involved problem. 


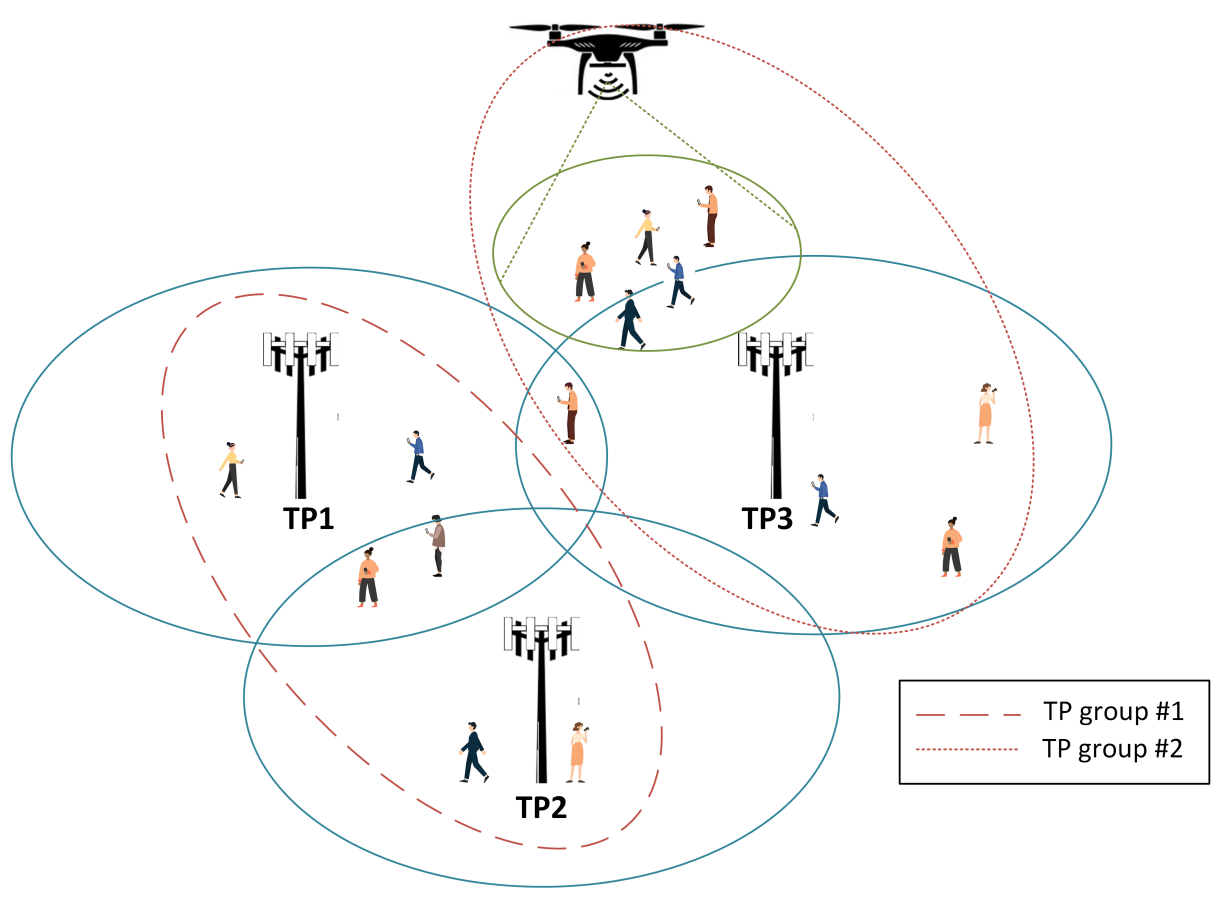

Figure 4.5: The VWA framework with a drone-BS.

The contributions of this study is twofold: First, we propose a long-term and interiorpoint based efficient TP grouping method. A convex problem is formulated, and the optimal solution is obtained by using the CVX tool of MATLAB [198]. The long-term, or average, TP groups are obtained to facilitate integration of drone-BSs, which is also performed on average channel models, rather than instantaneous decisions. Although this study focuses on downlink communications, the considered channel models are reciprocal. Second, we integrate a drone-BS into terrestrial system as an intrinsic component of the VWA framework, which is a novel system setting. In the literature either orthogonal resources are assumed for air- and ground-borne communications, or drone-BSs are positioned to minimize interference without any efficient coordination between terrestrial TPs and drone-BSs. On the contrary to many studies in the literature, we assume variable altitude and horizontal location, as well as complex air-to-ground channel models. In this study, drone-BS is not only positioned in 3D space to provide the most efficient service to the users, but also to obtain the most beneficial TP grouping. Note that in this case neither the TP group, nor the 3D position of the drone-BS are known. Therefore, we propose, first a sequential algorithm, where a drone-BS is efficiently placed in 3D space, and then the TPs are grouped. Furthermore, we propose methods for jointly placing the drone-BS and finding the optimal 
grouping by using powerful machine learning tools.

\subsubsection{System Model}

Consider $N$ terrestrial transmit points (TPs) in a set $\mathbb{T}=\left\{\mathrm{TP}_{1}, \mathrm{TP}_{2}, \ldots, \mathrm{TP}_{\mathrm{N}}\right\}$, and $M$ users in a set $\mathbb{U}$ are distributed with an arbitrary model in an area. The location of each user $j$ is known and represented by $\left(x_{j}, y_{j}\right)$. Then the received power of a user $j$ from a TP $i$ is calculated as

$$
P_{j i}^{R}=P_{i}^{T X}-L_{j i}+G_{a}+\sigma^{2},
$$

where, $P_{T X}, G_{a}$ and $\sigma^{2}$ represent the transmit power, antenna gain, and power of the Gaussian noise, respectively. The path loss from TP $i$ to user $j$ is indicated by $L_{j i}$, and adopted from [219] as

$$
L_{j i}=128.1+37.6 \log _{10}\left(d_{j i} \times 10^{-3}\right),
$$

where $d_{j i}$ is the horizontal distance between a user $j$ and TP $i$ in meters. Assume several TPs are grouped to form set $\mathcal{G}_{g}$ containing indices of the TPs in the group, and the subscript $g$ denotes the group index. Then, SINR for a user $j$ that is served by the TPs in set $\mathcal{G}_{g}$ becomes [217]

$$
\operatorname{SINR}_{j}=\frac{\sum_{i \in \mathcal{G}_{g}} P_{j i}^{R}}{\sum_{k \notin \mathcal{G}_{g}}^{N} P_{j k}^{R}+\sigma^{2}} .
$$

Accordingly, the spectral efficiency (SE) for the same user $j$ is

$$
\mathrm{SE}_{j}=\frac{1}{\left|\mathcal{G}_{g}\right|} \log _{2}\left(1+\operatorname{SINR}_{j}\right)
$$

where $|\cdot|$ denotes the cardinality of a set, i.e., number of TPs in the group.

Although TP cooperation can improve $\mathrm{SINR}_{j}$, SE may degrade due to large number of TPs in a group. Therefore, there is a trade-off between the number of TPs in a group, $\left|\mathcal{G}_{g}\right|$, and the interference reduction due to grouping. In order to reap the benefits of grouping as much as possible, we will be grouping the TPs based on user distributions, i.e., by grouping the TPs that cause most interference on users. However, before continuing with the details of the TP grouping methods, let us re-visit the air-to-ground channel characteristics very 
briefly for ease of reference.

\section{Air-to-Ground Channel Model}

We adopt the channel model in [27] as in the previous sections and briefly discuss the key aspects in this section. The air-to-ground channel differs from the terrestrial channel due to its higher chance of LoS connectivity. Hence, the air-to-ground channel model considers likelihood of LoS connectivity in path loss calculations. Moreover, the effect of the environment, e.g., density and average heights of the buildings, should also be considered.

The probability of having LoS for user $j$ depends on the altitude of the drone$\mathrm{BS}, h$, and the horizontal distance between the drone-BS and $j^{\text {th }}$ user, which is $r_{j}=$ $\sqrt{\left(x_{D}-x_{j}\right)^{2}+\left(y_{D}-y_{j}\right)^{2}}$ for the $j^{t h}$ user located at $\left(x_{j}, y_{j}\right)$ and the drone-BS at $\left(x_{D}, y_{D}\right)$. The LoS probability is given by [28]

$$
P\left(h, r_{j}\right)=\frac{1}{1+a \exp \left(-b\left(\arctan \left(\frac{h}{r_{j}}\right)-a\right)\right)},
$$

where $a$ and $b$ are constant values depending on the type of the environment, e.g., urban or suburban. In this setting, the altitude of the user, and the antenna heights of both the users and the drone-BS are neglected. Also, omnidirectional antennas are assumed to be utilized. Then the path loss expression becomes [28]

$$
L\left(h, r_{j}\right)=20 \log \left(\frac{4 \pi f_{c}}{c}\right)+20 \log \left(\sqrt{h^{2}+r_{j}^{2}}\right)+P\left(h, r_{j}\right) \eta_{\mathrm{LoS}}+\left(1-P\left(h, r_{j}\right)\right) \eta_{\mathrm{NLoS}}
$$

where $f_{c}$ is the carrier frequency $(\mathrm{Hz}), c$ is the speed of light $(\mathrm{m} / \mathrm{s}), \eta_{L o S}$ and $\eta_{N L o S}($ in $\mathrm{dB})$ are respectively the losses corresponding to the $\operatorname{LoS}$ and non-LoS connections depending on the environment. Equivalently, (4.7) can be written as

$$
L\left(h, r_{j}\right)=20 \log \left(\sqrt{h^{2}+r_{j}^{2}}\right)+A P\left(h, r_{j}\right)+B,
$$

where $A$ and $B$ are constants such that $A=\eta_{\mathrm{LoS}}-\eta_{\mathrm{NLoS}}$, and $B=20 \log \left(\frac{4 \pi f_{c}}{c}\right)+\eta_{\mathrm{NLoS}}$. Note that the path loss model presented here is a function of both horizontal and vertical positioning parameters, and it is a result of the $3 \mathrm{D}$ position of the drone-BS relative to the ground users. 


\subsubsection{Optimal TP Grouping and Drone-BS Integration strategies}

TP grouping is a fundamental step for VWA framework. Hence, we first discuss optimal TP grouping for terrestrial TPs, i.e., TPs at fixed locations. Afterwards, we propose two methods for drone-BS integration. The first method is sequential, where the drone-BS is positioned based on an efficient 3D placement method (Chapter 2), and then grouped with terrestrial TPs. Second, the positioning of drone-BS and grouping for TPs are obtained jointly by utilizing artificial intelligence methods, namely, Q-learning and double Q-learning.

\section{Optimal TP Grouping}

In this section, we propose long-term TP groups and find the optimal grouping for a given group size, TP and user locations based on average channel statistics given in Sec. 4.3.1. Note that "long-term" refers to non-instantaneous updates on TP groups. For instance, the study in [215] wraps the grouping algorithm around the scheduler, such that at approximately each scheduling interval, one of the overlapping TP groups is chosen. Instead, here the update of the TP groups can be performed over several scheduling intervals, and the frequency of updates can be determined with respect to the user mobility.

Assume that since the TPs in the same group will coordinate their transmission via methods such as beamforming and coordinated scheduling, they will cause minimal or no interference for the users they are serving. Therefore, assume a binary $N \times N$ matrix $\mathbf{B}=\left[\begin{array}{lll}\mathbf{b}_{1} & \mathbf{b}_{2} & \ldots \\ \mathbf{b}_{\mathbf{N}}\end{array}\right]$, where $\mathbf{b}_{\mathbf{i}}$ indicates if other TPs are in the same group with TP $i$ or not, such that if $b_{i k}=1$, then TP $i$ and $k$ are in the same TP group, and vice versa otherwise. Then, the problem for grouping TPs can be formulated as follows:

$$
\begin{gathered}
\underset{\left\{b_{j i}\right\}}{\operatorname{maximize}} \sum_{j \in \mathbb{U}} \sum_{i \in \mathbb{T}} \frac{P_{j i}^{R}}{\sum_{k \neq j}^{N} P_{k j}^{R} \times\left(1-b_{i k}\right)+\sigma^{2}} \\
\text { s. t. } \sum_{k}^{N} b_{i k} \leq G, \quad \forall i=1, \ldots, N, \\
\quad b_{i k} \in\{0,1\}, \quad \forall i=1, \ldots, N, \forall k=1, \ldots, N,
\end{gathered}
$$

where $G$ denotes the maximum group size. In the above form, this problem is non-convex 
due to the binary variables $\left\{b_{i k}\right\}$, and (4.9).

The non-convex (4.9) and binary constraint (4.11) makes the problem very hard to solve. In fact, we obtain a similar problem by modifying the formulation as follows: First, note that it is possible to relax the binary constraints and change the objective function with an equivalent form. When (4.11) is relaxed as $b_{i k} \in[0,1]$, and with the help of (4.10), the optimal solution will still be at the boundaries of the search space. It means that the BSs that interfere the most will have $b_{i k}=1$. This fact can be observed from the linearity of the denominator of (4.9). Then, consider that (4.9) is a linear-fractional, which is defined as the problem of maximizing (or minimizing) a ratio of affine functions over a polyhedron. Linear-fractional problems are quasiconvex in the feasible region, where the denominator is positive. They can also be converted into linear programs and be solved with interior-point based methods. However, we leave linear-fractional programming based re-formulation for future work. When the reciprocal of the objective function is taken and the problem is changed from maximization to minimization, we obtain a similar convex optimization problem that results in optimal TP groupings. Duality gap and showing the precise relation of the original and modified formulations are left for future work. Note that the $P_{j i}^{R}$ values are known, i.e., constants for BSs at known locations, and the only variable is $b_{i j}$. Hence, the reciprocal of the (4.9) becomes a linear function (noting that $b_{i} j$ are relaxed). The final formulation is as follows:

$$
\begin{aligned}
& \underset{\left\{b_{j i}\right\}}{\operatorname{minimize}} \sum_{j \in \mathbb{U}} \sum_{i \in \mathbb{T}} \frac{\sum_{k \neq i}^{N} P_{j k}^{R} \times\left(1-b_{i k}\right)+\sigma^{2}}{P_{j i}^{R}} \\
& \text { s. t. } \sum_{k}^{N} b_{i k} \leq G, \\
& \quad b_{i k} \in[0,1], \quad \forall i=1, \ldots, N,
\end{aligned}
$$

Note that this method is different than grouping the TPs based on their mutual distance, because the TPs are grouped based on the locations of the users. For instance, the TPs that have the most number of users at the edge are likely to be grouped with each other, even though there are other TPs in proximity (Fig. 4.5). On the other hand, (4.12) does not consider fairness, such that the optimal solution may be obtained by sacrificing some users with low-SINR. 


\section{Sequential Integration of a Drone-BS}

Recall that in Chapter 2, it is discussed in detail that placement of drone-BSs differ from placement of terrestrial TPs due to their mobility and agility. Neither the size nor the location of the coverage area of a drone-BS is known apriori. Despite the experiments and analysis performed for placement of terrestrial TPs, the location of a drone-BS is determined dynamically by considering current network situation. The network situation can include QoS requirements and locations of users, as well as locations and transmit powers of terrestrial TPs. In Section 2.1, we proposed an efficient 3D placement method, which is also adopted in this study as the first part of the sequential integration method. The efficient 3D placement algorithm optimally finds the coverage area with the minimum required size, i.e., energy expenditure, to cover the maximum number of users. That may be inline with the objective of this study, because increasing the performance of the maximum number of users can have the largest impact on (4.9), especially if those users are experiencing edge conditions (Fig. 4.5).

For ease of reference, we would like to briefly re-visit the key findings in Section 2.1. Let $R$ denote the radius of the area to be covered by the drone-BS, and $\beta$ denote the ratio of the altitude to the coverage region, such that

$$
\beta=\frac{h}{R}
$$

In fact, based on the channel model explained in Section 4.3.1, there exists an optimal $\beta$, i.e., $\beta^{*}$, which results in the largest possible $R, R^{*}$ for a $h$ [31]. Note that if a user is covered, $r_{i} \leq R$ must be satisfied, Furthermore, assuming maximum tolerable path loss as the desired QoS criteria, $R^{*}$ should be small enough the satisfy this property. Then, a user $\mathrm{j}$ is served by the drone-BS if $r_{i} \leq \Lambda$, where $\Lambda$ represents the $R^{*}$ for the environment and the maximum tolerable path loss value of the user. Then, after manipulations, a 3D placement problem with the objective of covering maximum number of users can be formulated as the following mixed integer quadratically-constrained optimization problem

$$
\begin{aligned}
& \operatorname{maximize}_{x_{D}, y_{D},\left\{u_{j}\right\}} \quad \sum_{i \in \mathbb{U}} u_{j} \\
& \text { s. t. } r_{j} \leq \Lambda+\left(1-u_{j}\right) K, \quad \forall j=1, \ldots, M, \\
& \quad x_{l} \leq x_{D} \leq x_{u},
\end{aligned}
$$




$$
\begin{aligned}
& y_{l} \leq y_{D} \leq y_{u}, \\
& u_{i} \in\{0,1\}, \quad \forall i=1, \ldots, M \text {. }
\end{aligned}
$$

where $u_{j}$ is a binary variable indicating whether a user $j$ is covered by the drone-BS or not, $K$ is a sufficiently large number to alleviate multiplication to comply with disciplined convex programming rules [198], and subscripts $u$ and $l$ denote upper and lower limits placement options, respectively. Note that neither $R$, nor $h$ are variables of (4.16). However, it is very easy to obtain $R$ from the location of the covered user with maximum $r_{j}$, and then $h$ can be derived by using $R$ and (4.15). These tricks reduce the number of variables and complexity of the problem, Thus, the problem can be easily solved by using interior-point optimizer of MOSEK. Note that the snapshot analysis in this thesis suggest many assumptions, among which the fixed association of users to TPs can be the most limiting along with the fixed transmit power of TPs. In fact, the modern wireless systems can more dynamically update user associations and perform advanced resource allocation. However, considering these factors results in a rather complex problem. The novel methodologies proposed in [220] and [221] can be the state-of-the-art solution methods for this type of problems. In particular, the study in [220] extends the classic fractional programming to solve continuous multiple-ratio convex-concave problems with a novel quadratic transformation. For instance, effectiveness of the proposed fractional programming method is demonstrated on a classic power control problem, where a set of single-antenna base stations each serve a single antenna user problem. In fact, the proposed method is shown to be easily generalized to a rate utility function. The study in [220] is particularly useful to consider power allocation with TP groupings, as well as updating the user associations. However, since the methods in [220] are proper for continuous problems, a disjoint approach between power allocation and TP grouping would need to be performed. Fortunately, the study in [221] advances [220] with the Lagrangian dual transform to solve discrete problems, such as user scheduling, which are more challenging. That is especially useful to improve this study to wrap the TP grouping, which is also a discrete problem, around scheduling. Since the proposed fractional programming algorithm outperforms the well-known weighted minimum mean-square-error algorithm that is used to solve similar problems, the methodology in [221] is promising to tackle with the increased complexity due to the VWA framework. Finally, the overall sequential algorithm for each snapshot is given in Algorithm 2. 


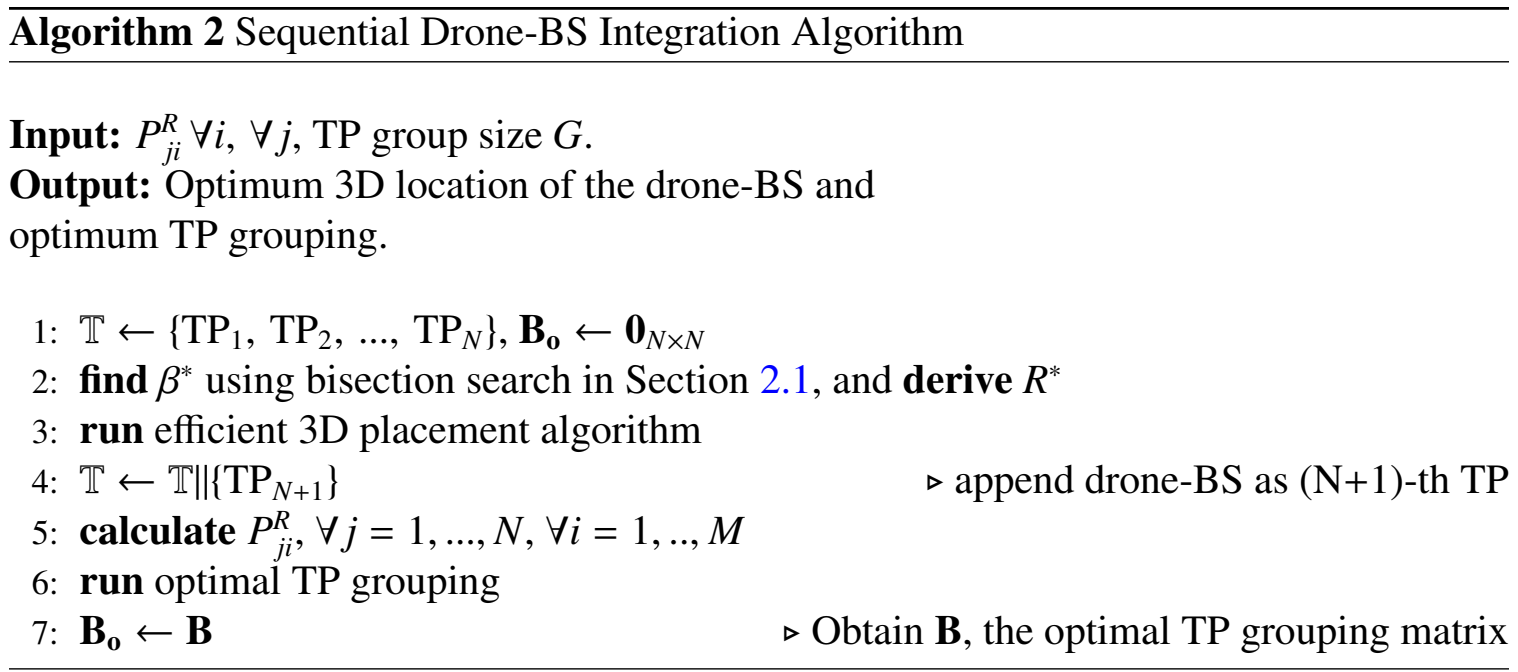

\subsubsection{Reinforcement Learning Based Integration of a Drone-BS}

In the previous section, TP groups are determined after the drone-BS is positioned, i.e., sequential integration. In this section, TP grouping and drone-BS placement will be performed simultaneously by using reinforcement learning (RL). It is a useful learning method that interprets the interactions between an agent and its environment to learn the best policies to follow for a successful outcome. It is particularly useful when learning has to be done on-the-go, i.e., no prior large data set, or environment model is available. Reinforcement learning is useful because when the positioning of the drone-BS is considered jointly with TP grouping, the received power values, $P_{j k}^{R}$ and $P_{j i}^{R}$ in (4.9), become non-linear functions of drone-BS location. Then, even with the relaxation of binary variables as discussed in Section 4.3.2, the problem remains non-linear and does not comply with disciplined convex programming rules [198]. Hence, utilizing learning-based methods or heuristic optimization methods is a viable option.

A branch of practical RL algorithms are temporal-difference (TD) learning algorithms. Q-learning is among the most popular TD learning algorithms, and it is used to solve droneBS related problems [41, 74, 122, 222]. Q-learning gets its name from the Q-function, $Q\left(S_{t}, A_{t}\right)$, which indicates the value of taking a certain action, $A_{t}$ at time $t$, in state, $S_{t}$. Here, the state refers to the observation on the environment, and action is what an agent can do. As a result, a reward is obtained as shown in Fig. 4.6.

In this study, Q-learning is especially chosen, because we formulate the joint drone-BS 


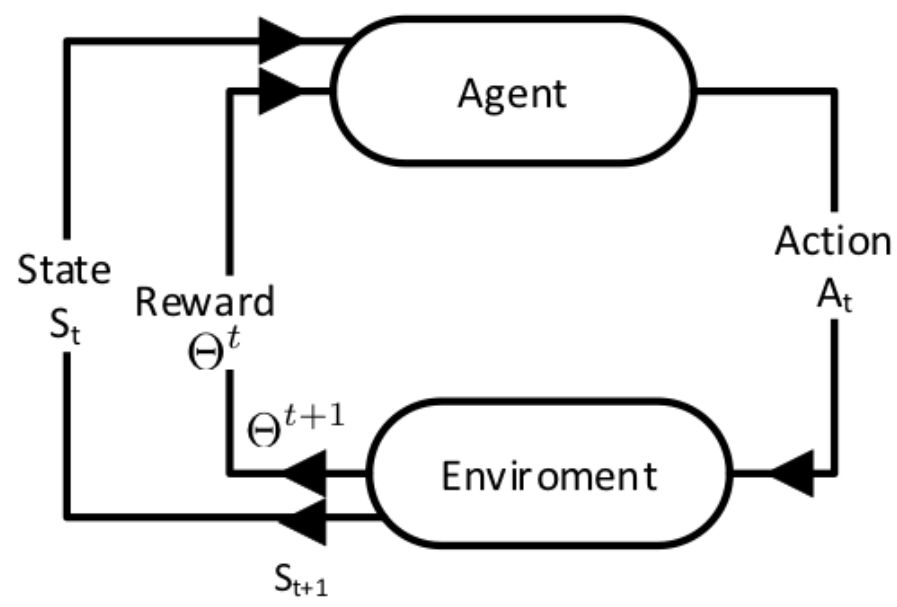

Figure 4.6: A generic RL scheme.

positioning and TP-grouping problem similar to "grid world" problems [223-225]. Although the characteristic grid world problem consists of a 2-D maze, here we have a 3-D maze as shown in Figure 4.7.

Similar to other grid world problems, states corresponds to the location of the drone-BS on the grid as well as its TP group, and actions are the movements of the drone-BS and TP grouping. In other words, two actions are simultaneously taken at each state: The position of the drone-BS for the next state is chosen and the TP groups are formed. In particular, the corresponding action can be shown as

$$
A_{t}=\left(\Delta \mathbf{x}_{t}, \Delta \mathbf{y}_{t}, \Delta \mathbf{z}_{t}, \mathbf{b}_{t}\right)
$$

where $\Delta \mathbf{x}_{t}, \Delta \mathbf{y}_{t}, \Delta \mathbf{z}_{t}$ correspond to the displacement of the drone-BS in $\mathrm{x}, \mathrm{y}$, and $\mathrm{z}$ dimensions, respectively. The actions in each dimension consists of increment or decrements based on the corresponding step size, i.e., $+\delta x,-\delta x,+\delta y,-\delta y,+\delta z,-\delta z$. This allows the drone-BS to discover the grid world to identify the most beneficial positioning. Also, $\mathbf{b}_{t}$ represents the update in TP groupings, i.e, which row of $\mathbf{B}$ is chosen for to group the droneBS with other TPs. Note that $\mathbf{B}$ is a binary matrix, where each column indicate a TP, and the non-zero element(s) in each row indicate the TPs that are grouped with the drone-BS. For instance, when the group size, $G=2$, the drone-BS can be grouped with only 1 of the 


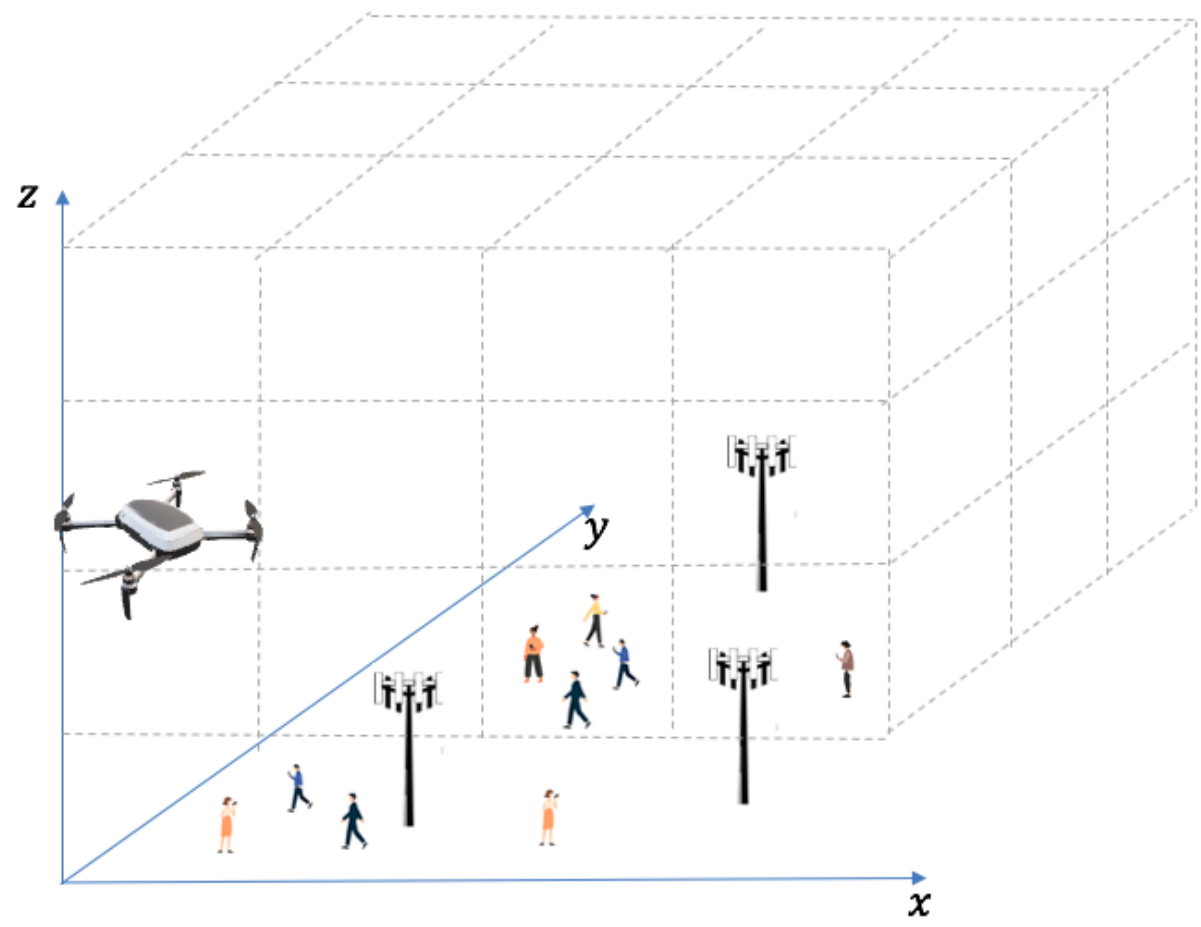

Figure 4.7: Grid world representation of the joint drone-BS placement and TP grouping problem.

TPs. Assuming 2 TPs, the TP-grouping options can be represented as follows

$$
\mathbf{B}=\left(\begin{array}{lll}
0 & 0 & 0 \\
1 & 1 & 0 \\
1 & 0 & 1 \\
0 & 1 & 1
\end{array}\right) .
$$

Assume here that the first two columns represent TPs and the last column represents the drone-BS. Then, the first two rows indicate that the drone-BS is not grouped with TPs. On the contrary, the last row indicates that the drone-BS is grouped with one of the TPs. Hence, action in this problem consists of two parts, because the 3D placement problem is jointly solved with TP grouping. Similarly, a state is represented as $S_{t}=\left[x_{t}, y_{t}, z_{t}, \mathbf{B}\right]$, where $x_{t}, y_{t}, z_{t}$ represent the location of the drone-BS in $\mathrm{x}, \mathrm{y}$, and $\mathrm{z}$ dimensions. These locations correspond to grid points. 
Then, a reward, $\Theta$, is obtained from the environment. Note that snapshot analysis are conducted and the air-to-ground channel model depends on average characteristic instead of instantaneous channels. Therefore, both user locations and channel conditions are assumed to be static at least until convergence. An appropriate reward for this problem is the improvement on the average spectral efficiency, which is calculated as

$$
\Theta^{t+1}=\frac{\sum_{j=1}^{M} \mathrm{SE}_{j}^{t+1}}{M}-\frac{\sum_{j=1}^{M} \mathrm{SE}_{j}^{t}}{M} .
$$

Note that $\mathrm{SE}_{j}^{t+1}$ is calculated by using (4.4) and (4.5) based on TP groups formed at each time step. Finally, the Q-function is updated as [223]

$$
Q\left(S_{t}, A_{t}\right) \leftarrow Q\left(S_{t}, A_{t}\right)+\alpha\left[\Theta^{t+1}+\gamma \max _{a} Q\left(S_{t+1}, A\right)-Q\left(S_{t}, A_{t}\right)\right]
$$

where $\alpha$ and $\gamma$ denote learning rate and discount factor, respectively. Actions are chosen with decaying $\epsilon$-greedy policy.

Q-learning has a maximization bias since both the update in (4.19) and $\epsilon$-greedy policy favors the largest $Q$ value [223]. A practical remedy to this problem is utilizing double $Q$-learning, which significantly alleviates the maximization bias problem by utilizing two $Q$-functions, $Q_{1}$ and $Q_{2}$. In this case, the update equation becomes

$$
Q_{1}\left(S_{t}, A_{t}\right) \leftarrow Q_{1}\left(S_{t}, A_{t}\right)+\alpha\left[\Theta^{t+1}+\gamma Q_{2}\left(S_{t+1}, \arg \max _{a} Q_{1}\left(S_{t+1}, A\right)-Q_{1}\left(S_{t}, A_{t}\right)\right] .\right.
$$

The decision on whether to update $Q_{1}$ or $Q_{2}$ is taken randomly at each episode, e.g., based on a coin toss. When $Q_{2}$ is selected for an update, $Q_{1}$ and $Q_{2}$ must be switched with each other in (4.20). On the contrary, the action selection at the beginning of each episode can be based on the summation, or average of both $Q_{1}$ and $Q_{2}$ values.

Note that the reward, $\Theta$, in (4.18) represents a different objective than the sequential integration. While $\Theta$ is focused on improving $\mathrm{SE}$, the sequential integration also focuses on exploiting the drone-BS as much as possible by serving maximum number of users. In order to investigate drone-BS exploitation, we propose the following reward function with normalized SE and served user values:

$$
\Theta_{2}^{t+1}=\frac{1}{M}\left(\frac{\sum_{j=1}^{M} \mathrm{SE}_{j}^{t+1}}{\max \left(\mathrm{SE}_{j}^{t+1}\right)}-\frac{\sum_{j=1}^{M} \mathrm{SE}_{j}^{t}}{\max \left(\mathrm{SE}_{j}^{t}\right)}+m_{t+1}-m_{t}\right)
$$




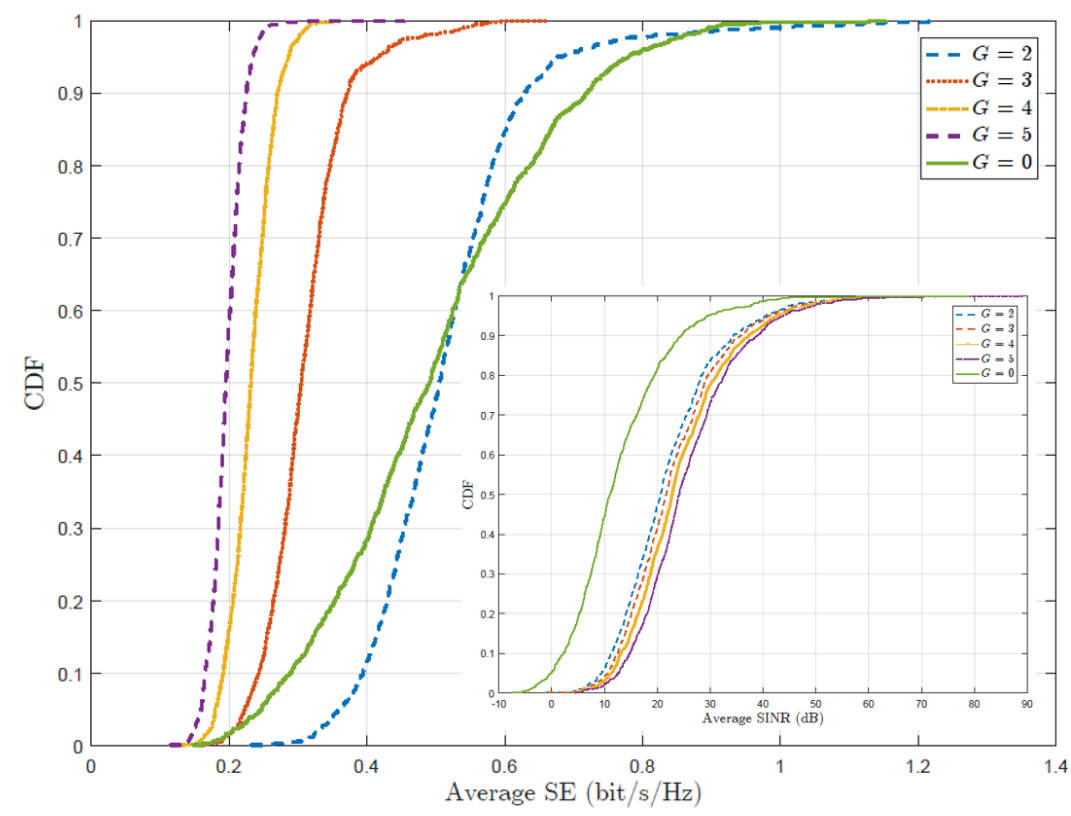

Figure 4.8: CDF of average SE and SINR with respect to TP group size.

where $m_{t}$ indicates number of users served by the drone-BS at time $t$. In $\Theta_{2}$, both the number of covered users and average SE has the same importance. However, it is possible to change the objective function based on the network benefit. For instance, if covering more users with tolerable service quality is more important than improving SE, e.g., due to a service level agreement, the weight for number of covered users can be increased. In the following section, several interesting simulation results and related discussions are provided.

\subsubsection{Simulation Results and Discussions}

First, we demonstrate the effectiveness of the TP grouping algorithm presented in Sec. 4.3.2 for different TP group sizes. 10 TPs and 200 users are randomly distributed in a $5000 \mathrm{~m} \times$ $5000 \mathrm{~m}$ area over 1000 Monte Carlo runs. Fig. 4.8 shows the CDF of the average SE for different group sizes, $G$, which indicate the number of TPs in a group. Note that $G=1$ indicates that there is only 1 TP in each group, i.e., VWA framework is not utilized. First, utilizing VWA increases average SE. However, the SE improvement due to increasing $G$ becomes less significant because of cost of cooperation in (4.5). A similar trend can be observed for SINR inside Fig. 4.8. Although the improvement in SINR is maintained, it 
Table 4.3: Simulation Parameters

\begin{tabular}{|c|c|c|c|}
\hline Parameter & Value & Parameter & Value \\
\hline$f_{c}$ & $2.5 \mathrm{GHz}$ & Noise power $(\sigma)$ & $-94 \mathrm{dBm}$ \\
\hline$P^{\mathrm{TX}}$ & $40 \mathrm{dBm}$ & $P_{\text {drone }}^{\mathrm{TX}}$ & $38 \mathrm{dBm}$ \\
\hline$G_{a}$ & $17 \mathrm{dBi}$ & $G_{a, \text { drone }}$ & $15 \mathrm{dBi}$ \\
\hline$h_{\max }$ & $2 \mathrm{~km}$ & $h_{\text {min }}$ & $20 \mathrm{~m}$ \\
\hline QoS & $100 \mathrm{~dB}$ & Area & $(2.25,25) \mathrm{km}^{2}$ \\
\hline$\alpha$ & 0.9 & $\gamma$ & 0.4 \\
\hline$a$ & 9.61 & $b$ & 0.16 \\
\hline$A$ & $1 \mathrm{~dB}$ & $B$ & $20 \mathrm{~dB}$ \\
\hline
\end{tabular}

becomes less significant with increasing $G$ as shown. Hence, Fig. 4.8 gives us two key messages: First, once some TPs causing most significant interference to each other are grouped, adding further TPs to the group size may not be significantly helpful. Second, considering that increasing the group size adds to the coordination complexity and is likely to increase overhead, determining the needed amount of improvement on average network performance is important. In other words, increasing the group size does not always mean that the overall network performance will be improved. In fact, determining optimal group sizes under various network conditions, e.g., centralized management vs. distributed management, under various user distributions and so on, is an interesting investigation on its own.

In the second set of experiments, 3 TPs and a drone-BS are utilized in a $1500 \mathrm{~m} \times 1500$ m environment. Table 4.3 summarizes simulation parameters along with air-to-ground channel parameters of the urban environment. Group size is chosen as 2 in light of the previous experiment. For each Monte Carlo run, the TPs and users are uniformly distributed. Fig. 4.9 shows the CDF of average SE with sequential integration, Q-learning, double Q-learning, double Q-learning with $\Theta_{2}$ in (4.21), and without using a drone-BS (only terrestrial TPs form virtual cells). There are two types of drones: Type - I drone-BS utilizes omni-directional antennas, which causes interference for the users that are outside of the coverage area of the drone-BS. Type - II drone-BS utilizes directional antennas, which prevents interference for the users outside of the coverage area. On the contrary to a Type - I drone-BS, a Type - II drone-BS does not contribute to the SINR of the users that 


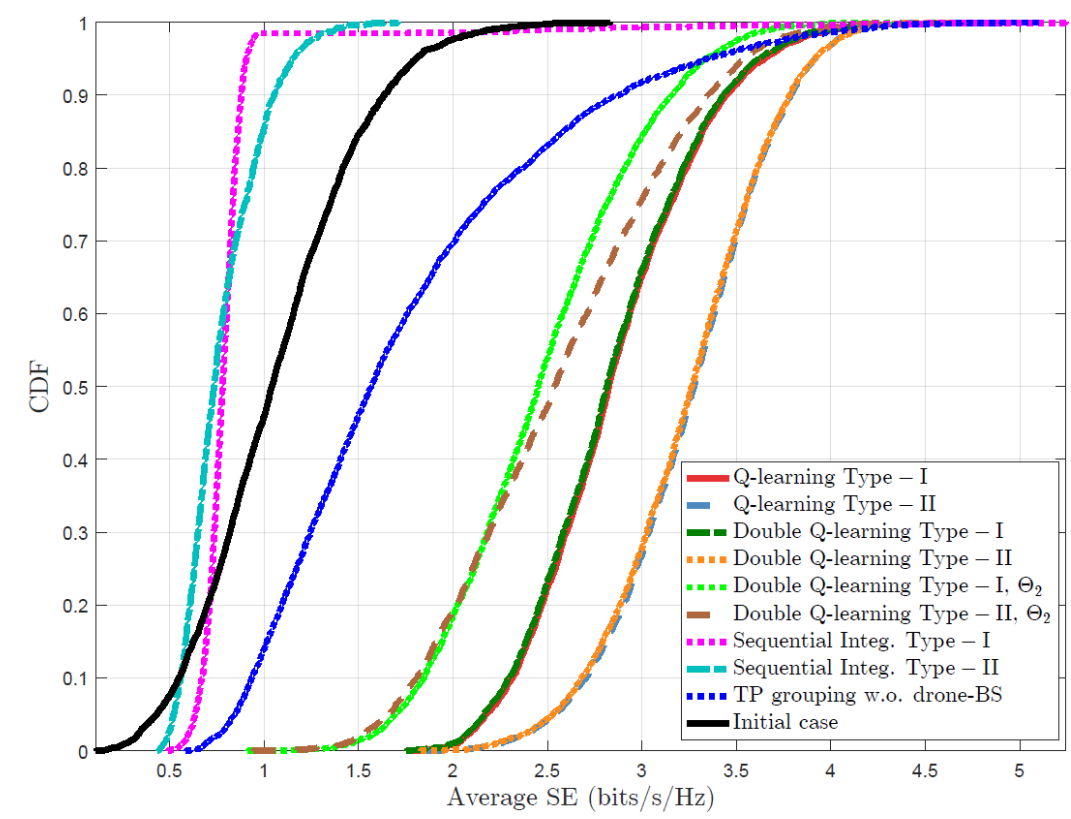

Figure 4.9: $\mathrm{CDF}$ of average $\mathrm{SE}$ with various integration methods.

are covered by TPs in the same group with the drone-BS. On the other hand, users of both type of drone-BSs suffer from interference caused by TPs of other groups.

Fig. 4.9 reveals rather interesting results: First, observe that the sequential integration method with both types of drone-BSs fail to improve SE compared to the initial case (no VWA and drone-BSs) and VWA with only terrestrial TPs, except for very low SE values. On the contrary, learning-based joint integration methods outperform all other methods significantly. That shows the importance of the integration methodology. Second, Type - II drone-BSs perform significantly better then Type - I drone-BS. The difference on average $\mathrm{SE}$ is up to $0.83 \mathrm{bits} / \mathrm{s} / \mathrm{Hz}$ on $50 \%$ of the time. Considering that users are uniformly distributed, i.e., no clustering, it is hard to distinguish a beneficial enough area with sufficient user density, if it ever exists. That is why strategies aiming at covering as many users as possible with the drone-BS, i.e., Q-learning with $\Theta_{2}$ and sequential integration, provides less improvement in SE compared to other methods with drone-BSs. However, Q-learning with $\Theta_{2}$ is more robust compared to sequential integration, since it considers both SE and number of covered users in (4.21), instead of considering only number of covered users as in the sequential integration.

On the other hand, Fig. 4.10 shows that sequential integration outperforms other methods when it comes to increasing utilization of the drone-BS in terms of number of covered 


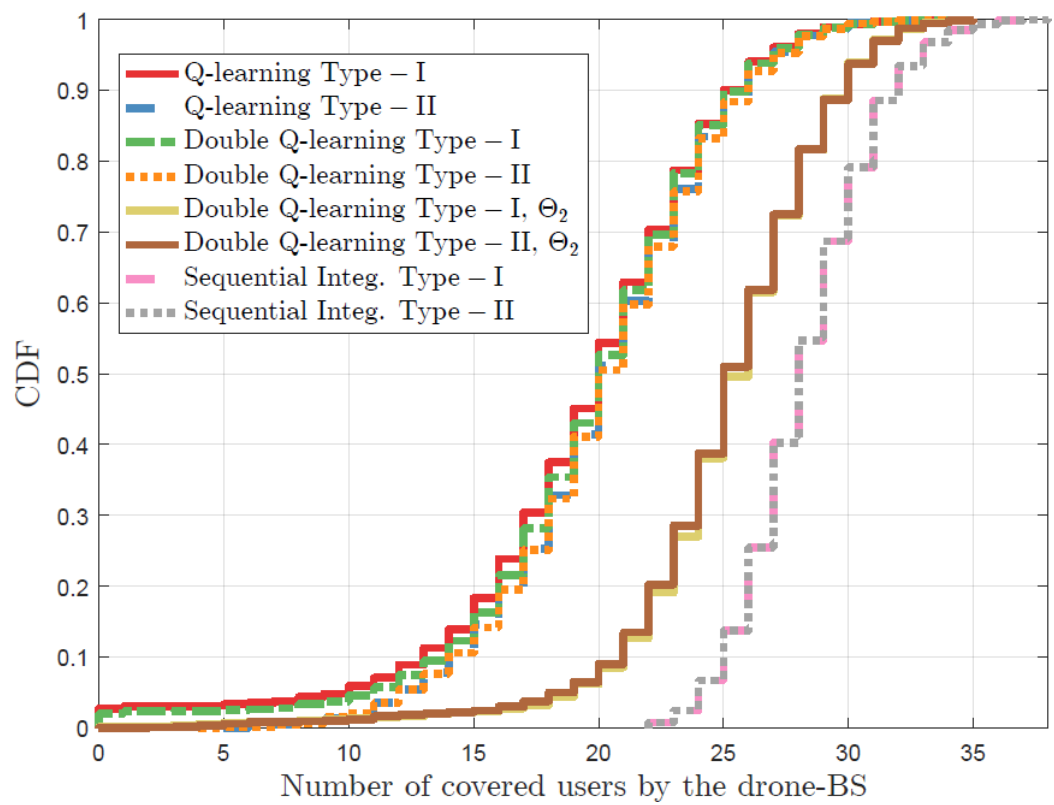

Figure 4.10: CDF of number of users covered by the drone-BS.

users. It is followed by Q-learning with $\Theta_{2}$, which equally considers improving SE and utilization. Comparing Fig. 4.9 and Fig. 4.10 reveals that different integration methods can be utilized based on the target benefit of the network. For instance, sequential integration is particularly useful if the network needs to overcome congestion, whereas learning-based method with $\Theta_{1}$ is more useful when increasing average SE, e.g., for improving average throughput, is vital. Finally, Fig. 4.11 shows convergence times of different learning algorithms. Note that convergence of Q-learning is discussed and proved for several cases in $[223,226,227]$, and there are several studies that consider number of iterations until convergence to discuss convergence characteristics, such as [228]. As expected, double Q-learning provides faster convergence than Q-learning for the same objective function. However, when $\Theta_{2}$ is the objective function, convergence is slightly slower than other cases, because the problem becomes more complex.

\subsection{Summary and Discussions}

In this chapter, we began with exploring drone-BSs from a system-level view. We discussed their opportunities, challenges, drone types, and business models. We highlighted that efficient integration of drone-BSs into wireless networks is one of the biggest challenges 


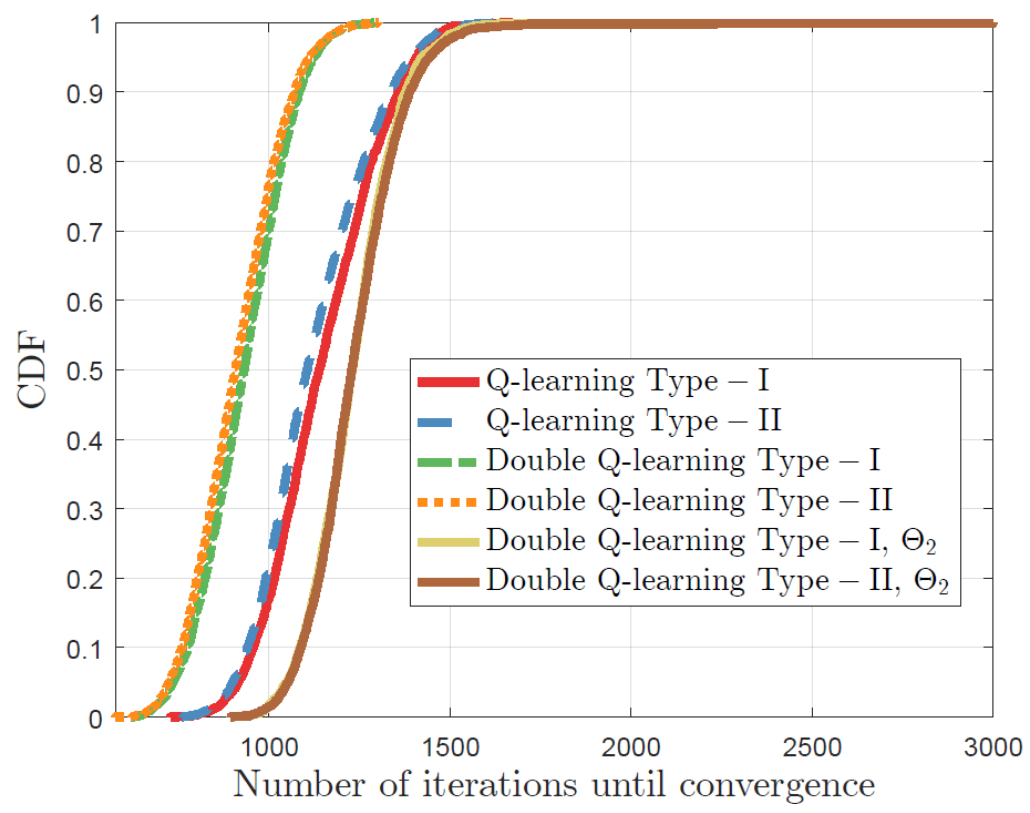

Figure 4.11: CDF of number of iterations until convergence.

to benefit from drone-BSs as much as possible. For that purpose, we first investigated an integration method based on business models, i.e., multi-tenant wireless networking. Then, we provided an operational study by investigating various integration techniques when virtual wireless access is implemented in wireless networks. With these studies we show that the integration method affects the performance gain significantly. Moreover, there is not a universal method that can address all the target benefits at the same time. Hence, determining the target benefit for the network and choosing the proper integration method has crucial importance. In the following chapter, we will investigate whether the discussed integration models can be supported by the upcoming $5 \mathrm{G}$ networks. 


\section{Chapter 5}

\section{Is 5G Ready for Drones?}

So far we have investigated airborne communications in wireless networks from a research point of view. We have discussed drone-BS types and applications, operational details, such as 3-D placement, and integration methods. This chapter aims to provide a sanity check for the research activities by reviewing 5G Phase-I standards. In fact, there are two main questions regarding the interaction of drones with wireless networks: First, how wireless networks can support personal or professional use of drones. Second, how drones can support the wireless network performance, i.e, boosting capacity on-demand, increasing coverage range, enhancing reliability and agility as an aerial node. From a communications perspective, this chapter categorizes drones of the first case as mobile-enabled drones (MEDs), and drones of the second case as wireless infrastructure drones (WIDs) including drone-BSs. We investigate both the MED and WID cases within the realistic constraints of 5G. In both categories, unless otherwise stated, e.g., high-altitude platforms, the preferred type of drones is similar to medium-sized devices with moderate capabilities, e.g., quadrators or fixed-wing unmanned airplanes [2,229]. Note that WIDs are a subset of networked flying platforms, where the term also covers non-terrestrial networks ${ }^{1}$ (NTNs) (TR 38.811).

For discussing the communication aspects of MEDs and WIDs, this section has two aims: First, highlighting crucial information about 3GPP standardization which serves as a basis for research on the integration of drones into contemporary and prospective mobile networks. Second, discussing solutions for open issues, either via applications of current

\footnotetext{
1"Networked flying platform," Mar 2018, Accessed: 2019-08-25. [Online]. Available: https://en. wikipedia.org/wiki/Networked_flying_platform .
} 
methods, or by providing suggestions towards further enhancements. Throughout this section, we help interested researchers navigate the standardization documents by pointing relevant references. In particular, one of the aims of this study is to serve as a bridge between the researchers in academia and industry by presenting a comprehensive discussion of the current trends in $5 \mathrm{G}$ standardization activities.

\subsection{Drones and Mobile Networks}

Drones have diverse capabilities and scales from nano-robots to aircrafts with larger wingspans than a Boeing [2]. That variety reflects in terminology by creating a number of terms such as Unmanned Aerial Vehicles or Systems (UAV or UAS, respectively). Compared to the other terms, "drone" is a vague one; it means a remotely controlled device which can operate in any medium (air, water, or land). However, "drone" is also the most popular and compact term. There are ongoing standardization activities in 3GPP for providing enhanced wireless connectivity to personal and commercial drones via mobile networks, i.e., MEDs or aerial-UEs (AUEs). However, enhancing mobile networks via utilization of drones providing direct or indirect connectivity to other UEs, i.e., WIDs, is a fairly new scenario. Note that the same equipment may be utilized both as a MED and as a WID. In this case, MED and WID correspond to different operational modes, rather than devices.

\subsubsection{Mobile-Enabled Drones}

Drones in this category are UEs from the perspective of wireless networks, i.e., a business client, which may also include private drone networks. Currently, drones are being used in vast applications, including inspections and surveys, transport and logistics, and surveillance and monitoring. In TR $36.777^{2}$ a maximum altitude of $300 \mathrm{~m}$ and a maximum speed of $160 \mathrm{~km} / \mathrm{h}$ is determined for both rural and urban scenarios of such drones. These use cases should not be confused with the "commercial air-to-ground" in TR 38.913, where

\footnotetext{
${ }^{2}$ All TR and TS documents are 3GPP technical report and specification documents, respectively. The acronym of "3GPP" is omitted for brevity.

${ }^{3}$ For $1 \mathrm{GHz}$ bandwidth at $26 \mathrm{GHz}$ band.
} 


\begin{tabular}{|l|l|l|l|l|}
\hline QoS requirements & URLLC & eMBB & MED Command\&Control & MED Application \\
\hline User Plane Latency (UL/DL) & $0.5 \mathrm{~ms} / 0.5 \mathrm{~ms}$ & $4 \mathrm{~ms} / 4 \mathrm{~ms}$ & $50 \mathrm{~ms} /$ Unspecified & $50-400 \mathrm{~ms}$ \\
\hline Reliability (1-Block Error Rate) & $1-10^{-5}$ & $1-10^{-3}$ & $1-10^{-3}$ & Unspecified \\
\hline Peak Data Rate (UL/DL) & $1.75 \mathrm{Gbps} / 6.5-13 \mathrm{Gbps}^{3}$ & $1.75 \mathrm{Gbps} / 6.5-13 \mathrm{Gbps}$ & $60-100 \mathrm{kbps}$ & $50 \mathrm{Mbps}$ \\
\hline
\end{tabular}

Table 5.1: QoS requirements for URLLC, eMBB and MED scenarios in 3GPP RAN documents.

the term means providing mobile connectivity services to humans and machines on-board commercial aircrafts.

Traditionally, drones use unlicensed links to communicate with a ground control station (GCS), or drone pilots [7]. However, unlicensed links have limited reliability and range due to the propagation impairments of remote control signals in beyond-visual-LoS (BVLoS) operations, which are mostly prohibited, which are mostly prohibited, e.g., in USA by the Federal Aviation Administration. Contrarily, mobile networks can enable BVLoS operations thanks to their wide coverage and reliability. Moreover, secure communications (TS 33.501), capability for lawful intercept (TS 33.107), location verification, and trusted identification (TR 33.899) are side benefits that can be obtained via current and next-generations of standardized mobile networks [230].

MEDs establish two types of links with a GCS: First, the command\&control link is used for remote piloting, telemetry data, identity, navigation, and similar (TR 36.777). Although telecommand and telemetry links come under a single non-payload communications umbrella, remote piloting may require video transmission to provide a near-there feeling to the pilot. Since many regulatory bodies do not approve fully autonomous drones (due to operational risks), and drones can operate only in semi-automated fashion under the supervision of drone pilots, command\&control links are critical [7]. Second type of link established with a GCS is the application link that delivers information, such as sensor data, video, audio, and images. Note that the application link mostly requires payload communication capabilities. On the other hand, application data is less critical than command\&control for many cases. Therefore, both throughput and reliability requirements vary (Table 5.1). 


\subsubsection{Wireless Infrastructure Drones}

Differently from MEDs, WIDs serve to enhance network capabilities, e.g., by increasing coverage or capacity. WIDs can be classified based on their functionalities and requirements:

- Aerial- or Drone-BSs: Drone-BSs serve as aerial-nodes with some or all functionalities of BSs, (e.g., an aerial-eNB, or an aerial distributed unit (DU)) creating dronecells [2]. For drone-BSs, both wireless backhaul and fronthaul may be required [40], or either one of them may be provided via tethering. Downlink and/or uplink radio access can be licensed or unlicensed. Drone-BSs have various moving patterns, e.g., hovering, rotating, floating, following a specific route, or landing on suitable locations (e.g., top of buildings). These patterns depend on environmental conditions, machinery, and communication requirements.

- Aerial-relays (AR):ARs can be deployed by users or operators. In the former case, unlicensed spectrum can be used for the links between user and AR, and the AR acts as a UE for the mobile networks. Operator-deployed ARs can be more sophisticated, and should be integrated appropriately. They can act as intermediate hops for integrated access and backhaul (IAB) (Sec. 5.2.4), or simple analog repeater with up/down converters.

- Aerial backhaul/fronthaul providers: Drones form NTNs providing an aerial transport network (ATN). The interest in ATNs is rapidly increasing ${ }^{4}$. While both licensed and unlicensed solutions are possible, the hybrid ones seem the most efficient. TR 38.811 considers satellites as part of the NTN of 5G networks. However, they are not considered here, as the altitude of even low-orbit satellites is above the limits within which small cell/relay-like operations are feasible.

\footnotetext{
"FCC authorizes SpaceX to provide broadband satellite services," Mar 2018, Accessed: 2019-0825. [Online]. Available: https://transition.fcc.gov/Daily_Releases/Daily_Business/2018/ db0329/DOC-349998A1.pdf.
} 


\subsection{Progress of 5G Standardization and What It Means for Drones}

In this section, selected capabilities of 5G networks are discussed based on Rel-15 $5^{5}$ developments in RAN (RAN1, RAN2, RAN3) and SA working groups (SA2, SA5). This section is organized as follows: First, the concept of slicing is explained. Then, 5G RAN topics which are primarily important are investigated. These topics include standardization activities for MEDs, architectural roles for MEDs and WIDs, integrated access and backhaul studies, and licensed/unlicensed options from the perspective of drones. Finally, 5G core and $5 \mathrm{G}$ network management studies are discussed in terms of solutions they can promise for the integration of drones.

\subsubsection{Slicing}

Slicing enables service-oriented configuration of wireless networks in a flexible and agile manner [231]. Hence, the network is arranged to support different drone services, e.g., a slice configured for application links of MEDs, or a slice to isolate the traffic of WIDs. (Fig. 5.1(a)). SDN and NFV technologies are key enablers for slicing in 5G.

For SA2 and RAN, slice is a "logical network that provides specific network capabilities and network characteristics". However, SA5 considers "managed network slice instances" (NSIs) with various constituents, i.e., managed network slice-subnet instances (NSSIs), e.g., with respect to domain (RAN, core), or location (Ottawa, Toronto, etc.). Fig. 5.1(b) shows two 5G-core slices isolating the traffic and network resources of a WID and gNB. Note that slices have shared (e.g., AMF, SMF) and non-shared components (e.g., user-plane functions, NEF). From a management perspective, 5G-core slices are each an NSSI, where an end-to-end NSI contains RAN-NSSI and CN-NSSI (Sec. 5.4) (Fig. 5.1(a)).

RAN supports slicing, e.g., by allowing hard slicing and resource isolation between slices, QoS differentiation within a slice, and awareness of slice identification. Some further developments in RAN and their meaning for drones are discussed next.

\footnotetext{
${ }^{5}$ All releases in this chapter are 3GPP releases, however, the acronym of "3GPP" is omitted for brevity.
} 


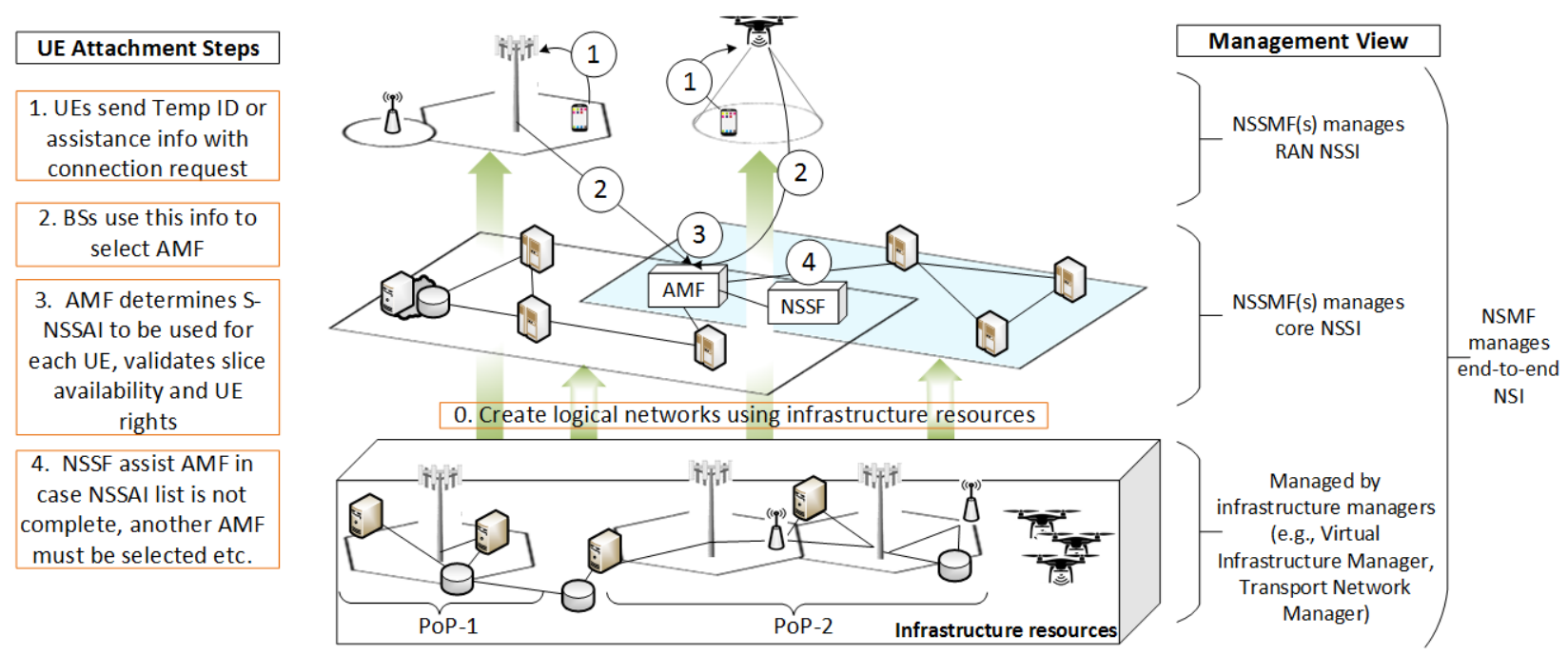

(a)

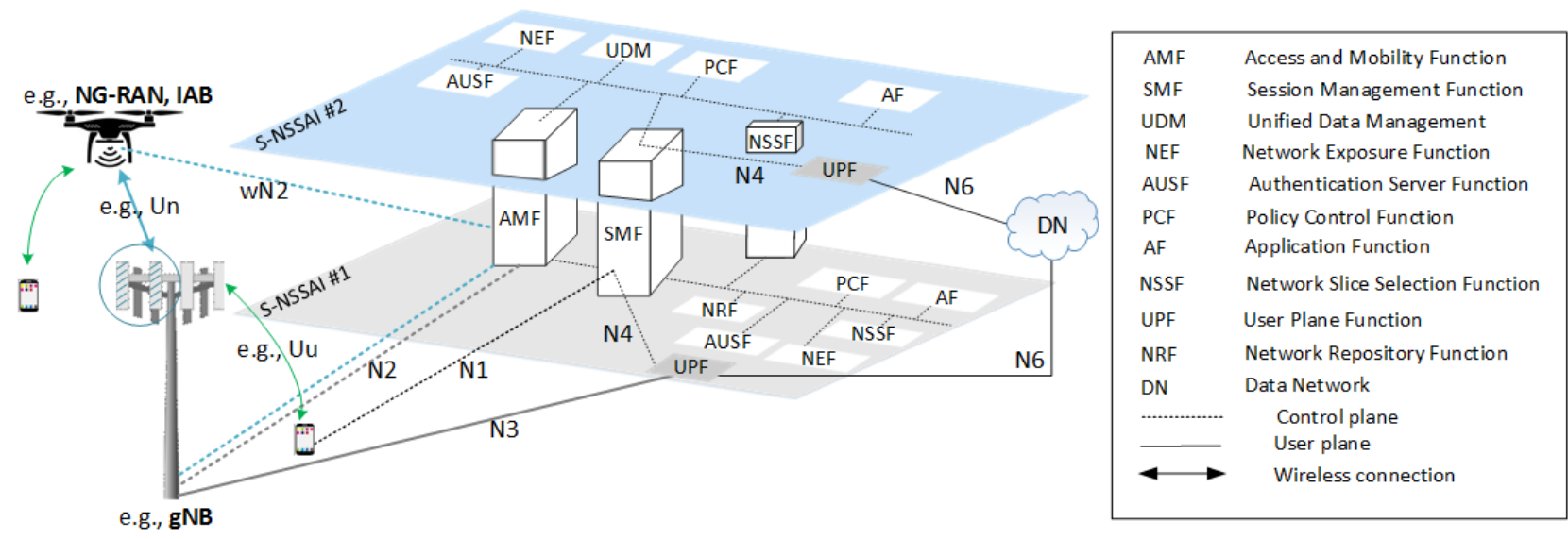

(b)

Figure 5.1: Wireless networks with slicing and integration of WIDs: (a) Creation of logical networks from infrastructure pools. Some initial UE-attachment steps are highlighted. (b) Interfaces and service-based 5G-core slices. A WID-specific 5G-core slice may include common and non-shared NFs; the upper WID slice has isolated resources from the gNB slice below. Circle depicts gNB resources reserved to provide backhaul and/or fronthaul to the WID (hard-slicing in RAN). 


\subsubsection{Standardization for MEDs in 5G RAN}

The most comprehensive study for MEDs is in TR 36.777 where definitions, scenarios, performance requirements and metrics, interference problem and potential remedies are discussed. Data and setups regarding field trial results, system-level and mobility evaluations, and fast fading models are going to be provided in future versions.

Performance metrics in TR 36.777 are presented in Table 5.1 in comparison to QoS requirements of URLLC and eMBB cases (TR 38.918). Table 5.1 shows that MEDs require 100 times less reliability than URLLC, and 200 times less peak data rate than eMBB.

Mostly RAN working groups perform standardization activities regarding MEDs, whereas SA2/5 has no specific study for MEDs. Although MEDs are considered among IoT, URLLC and eMBB scenarios (TS 23.501, Sec. 5.3), considering Table 5.1, such categorization may be imprecise.

\subsubsection{Architectural Roles in 5G RAN}

5G architecture benefits from decoupling roles, capabilities, and functionalities of network elements to provide flexible and diverse technical solutions that can be tailored for specific needs. Hence, there are various integration options for WIDs with varying levels of flexibility, cost, security, and complexity. There are 3 types $^{6}$ of RAN nodes in Rel-15:

- eNB: E-UTRA user-plane and control-plane protocol termination towards UE, connected to EPC core via S1 interface (TS 36.300)

- ng-eNB: E-UTRA user-plane and control-plane protocol termination towards UE, connected to 5G-core via NG interface (TS 38.300).

- gNB: New radio (NR) user-plane and control-plane protocol termination towards UE, connected to 5G-core via NG interface (TS 38.300).

\footnotetext{
${ }^{6}$ There is also en-gNB, with NR user-plane and control-plane protocol termination towards UE, connected to EPC core via S1 interface. Although en-gNB seems to be a natural evolutionary step, it is not standardized by $3 \mathrm{GPP}$.
} 


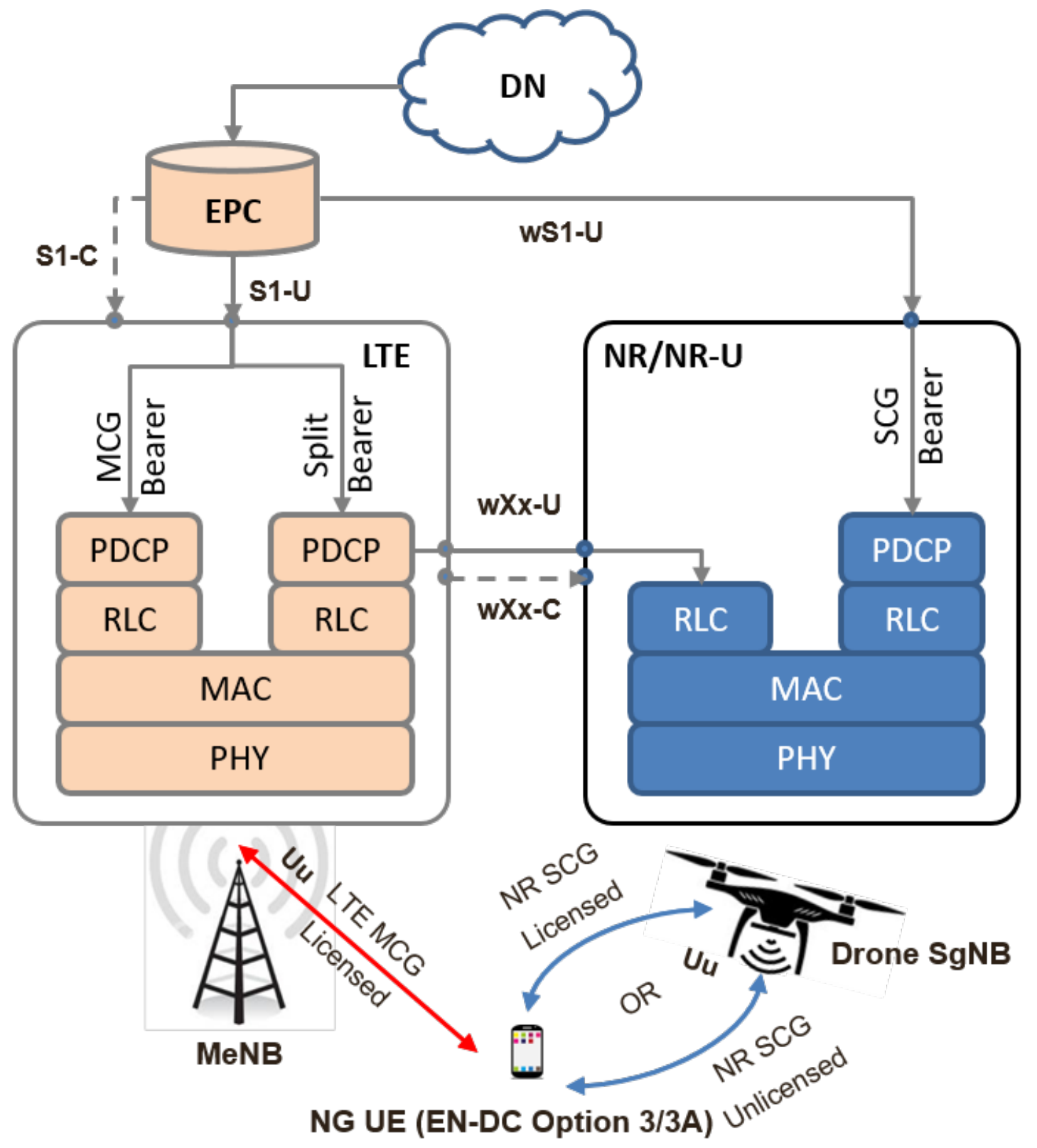

Figure 5.2: Combined protocol stack architecture and simplified reference point model for licensed/unlicensed WID integration with Drone-SgNB using NR/NR-U radio in EN-DC option 3/3A.

Also, NG-RAN node refers to gNB or ng-eNB. Until full-fledged 5G is deployed, and 5G UEs become widely available, inter-working between $4 \mathrm{G}$ and $5 \mathrm{G}$ is necessary to provide seamless service. Inter-working can be in standalone or non-standalone mode. In the 


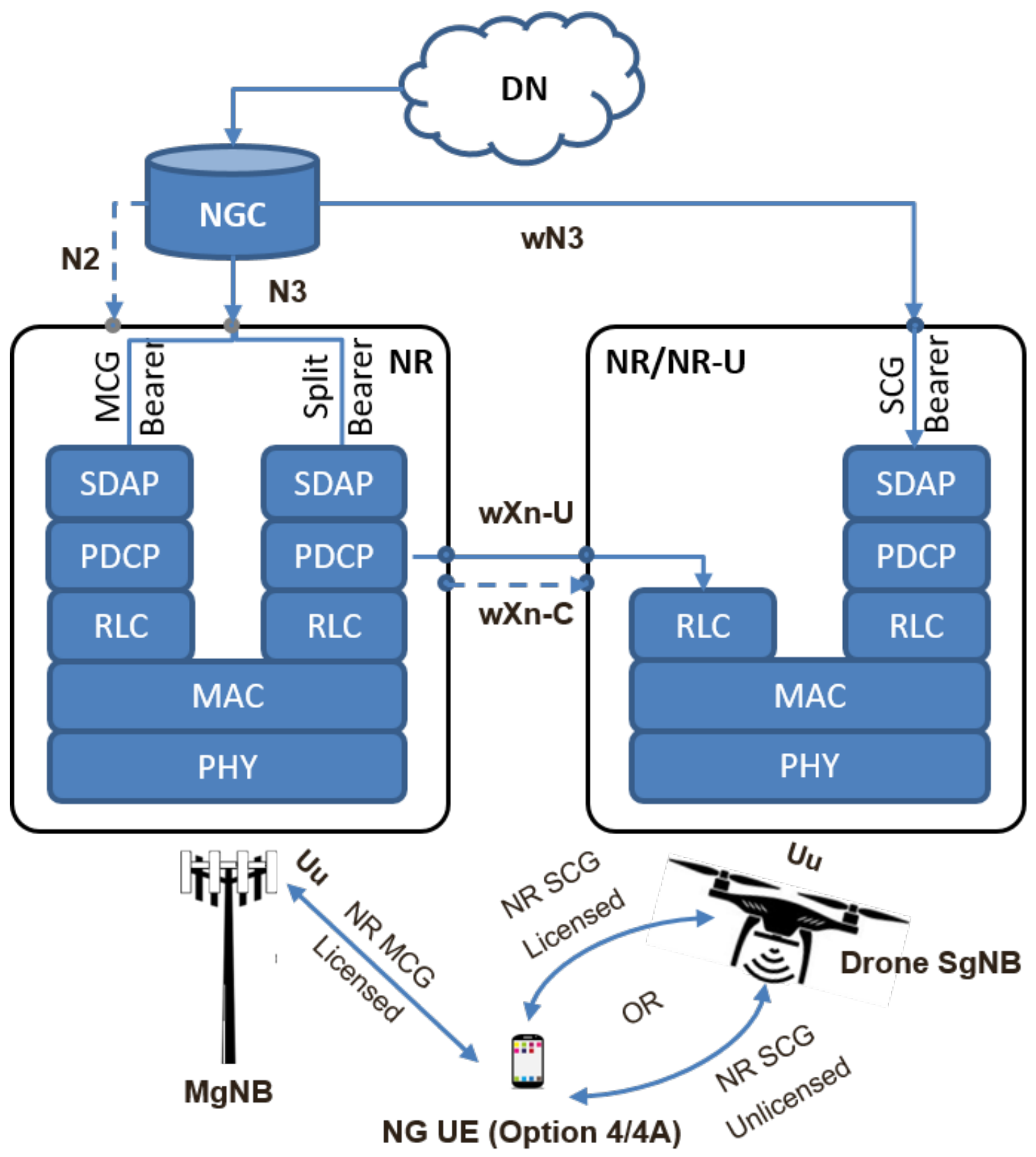

Figure 5.3: Combined protocol stack architecture and simplified reference point model for licensed/unlicensed WID integration with Drone-SgNB using NR/NR-U radio in NE-DC option 4/4A.

standalone mode, UEs that are compatible with LTE and 5G can access to either one of the cells [232], which requires CN solutions.If non-standalone, a WID acts as an aerial-NR node providing high data rate user-plane communications to the UE, while control-plane 


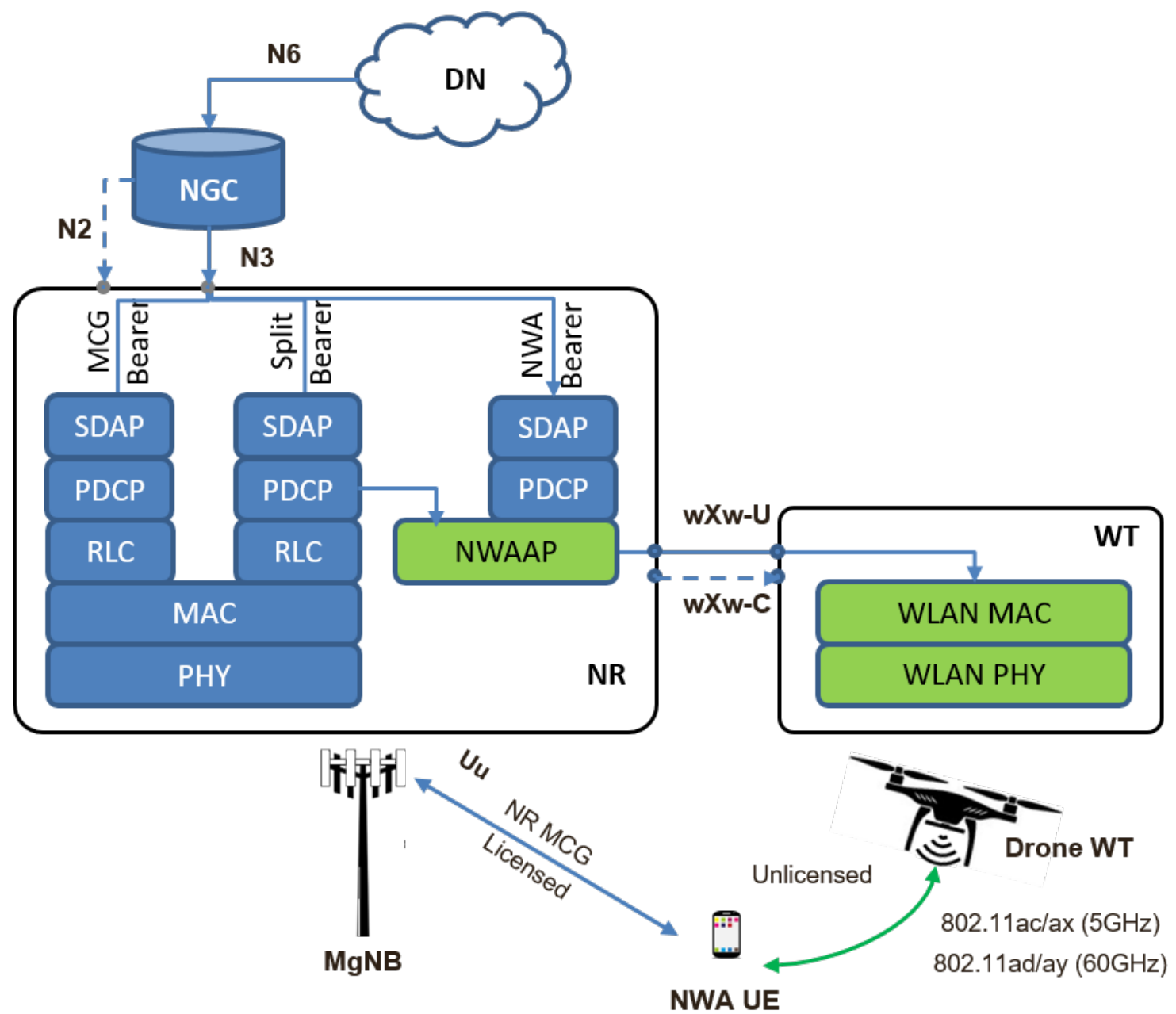

Figure 5.4: Combined protocol stack architecture and simplified reference point model for licensed/unlicensed WID integration with WLAN termination drone in NR-WLAN aggregation.

is handled by the terrestrial-eNB. Note that the NR node is only connected to eNB and service-gateway or user-plane functions in this case (Fig. 5.2 and Fig. 5.3, Sec. 5.2.5). If standalone, WIDs can be ng-eNBs or gNBs with wireless backhaul (Fig. 5.6, Fig. 5.7) while UEs that are compatible with LTE/LTE-A and 5G can access either one of the cells [232].

BSs can be deployed with hierarchy as master and secondary nodes, in case of MultiRAT dual connectivity (DC) (TS 36.340). Either ng-eNB or gNB can be the master node of the other, known as NGEN-DC or NE-DC, respectively (TS 37.340). Similarly, EN-DC indicates that an eNB is the master node of an NG-RAN node. Although, in principle, all DC options can be viable for WIDs (Fig. 5.2 and Fig. 5.3), it is more efficient for WIDs to 


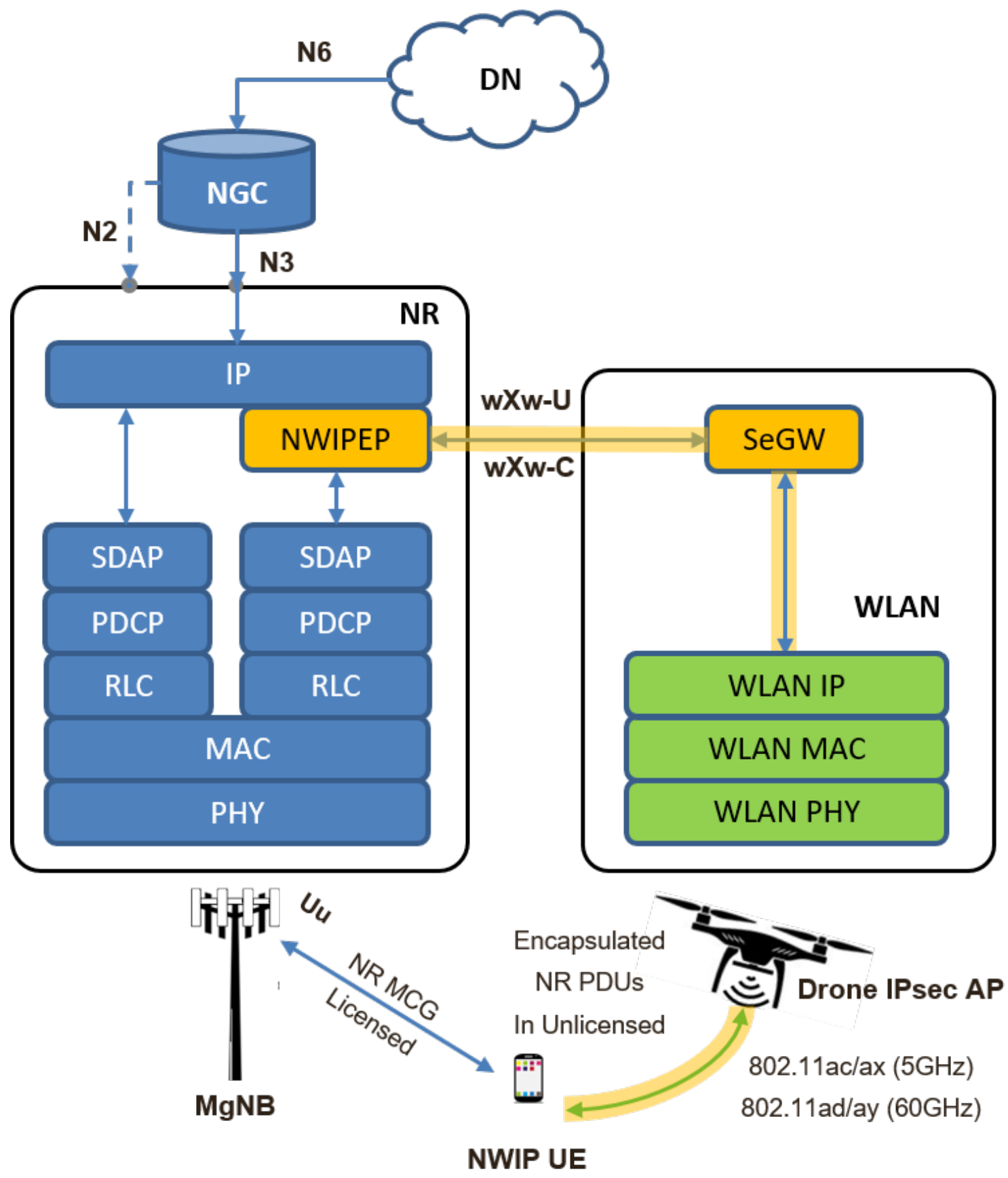

Figure 5.5: Combined protocol stack architecture and simplified reference point model for licensed/unlicensed WID integration with Drone IPsec Access Point in NR-WLAN integration using IPsec tunnel.

be secondary nodes to reduce complexity, and prevent excess control-plane latency due to wireless fronthaul/backhaul. 
Furthermore, the UE can be configured to receive only data transmission over the WIDSCG while L1/L2 control signaling can be received with larger coverage and more reliability over the MSG of a Macro/Micro NB. In particular, this could be a typical configuration when the WID backhaul and/or access links are in the unlicensed band wherein channel availability and interference levels are not guaranteed.

ANs can be deployed with hierarchy as master and secondary nodes, in case of Dual Connectivity (DC). A UE in RRC_CONNECTED state is configured with a Master Cell Group (MCG) and a Secondary Cell Group (SCG) (3GPP TS 36.300). For Rel-15 and onward, DC is generalized for multiple RATs, hence the name MR-DC (3GPP TS 37.340). Either ng-eNB or gNB can be the master node of the other one, resulting in NGEN-DC or NE-DC, respectively. Whereas when an eNB is the master node of an NG-RAN node, it is called EN-DC. All options can be viable for WIDs, as shown in Fig 5.2 and Fig 5.3. However, WIDs can be more appropriate as secondary NBs, since it reduces the complexity of the drone node, and avoids excess control-plane latency due to the wireless backhaul connection. Furthermore, the UE can be configured to receive only data transmission over the WID-SCG while L1/L2 control signaling can be received with larger coverage and more reliability over the MSG of a Macro/Micro NB. In particular, this could be a typical configuration when the WID backhaul and/or access links are in the unlicensed band wherein channel availability and interference levels are not guaranteed. Accordingly, WIDs can be aerial-DUs with limited functionalities of a central unit (CU) (Fig. 5.6 through Fig. 5.9). The CU/DU split is proposed to enable and enhance the cloud-RAN technology via several split options (TR 38.801) that allow arranging centralization vs distribution of control and capabilities depending on each situation of wireless networks, e.g., supporting large number of UEs, abundance or scarcity of bandwidth, delay tolerant or sensitive applications, and expanding coverage range. While the main assumption is a one-to-one connection between gNB-DU and gNB-CU, a gNB-DU can be connected to multiple gNB-CUs for resiliency.

Out of 8 split options, option-2 and option-3 are mostly debated (Fig. 11.1.1-1, TR 38.801). In option-2, radio link control (RLC) is with DU, and packet data convergence protocol (PDCP) is with $\mathrm{CU}$; radio resource control protocols for signaling radio bearers and service data adaptation protocol (SDAP) for data radio bearers are also with CU (TS 38.401). There is no split for signaling radio bearers, if option-2.1 is used (3C-like split); wherein DUs need to have all layers and required capabilities of control-plane. The main 


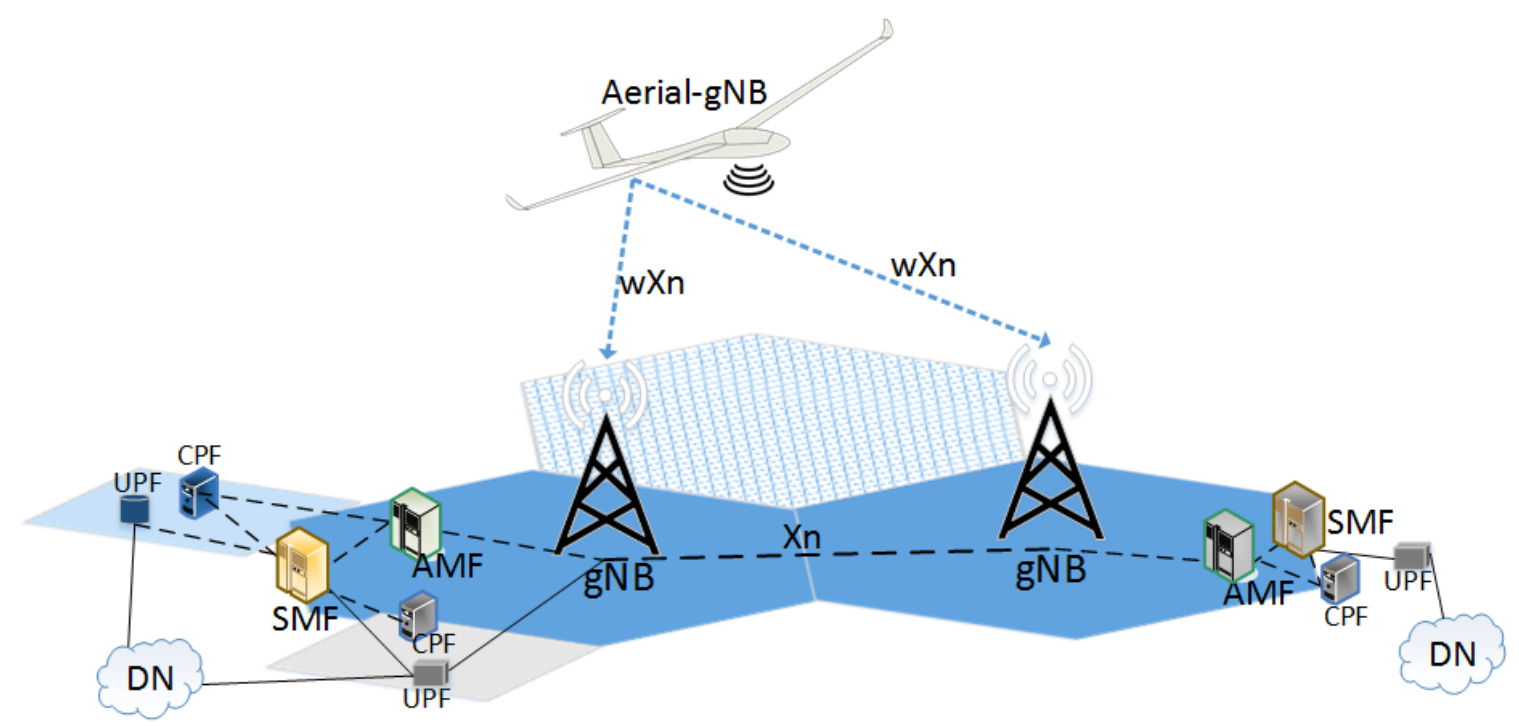

Figure 5.6: Architecture option for WIDs where a standalone aerial-gNB with a specific $5 \mathrm{G}$-core-slice for WID-originated traffic (blue slice) is utilized. Interfaces with potential enhancements are marked with "w".

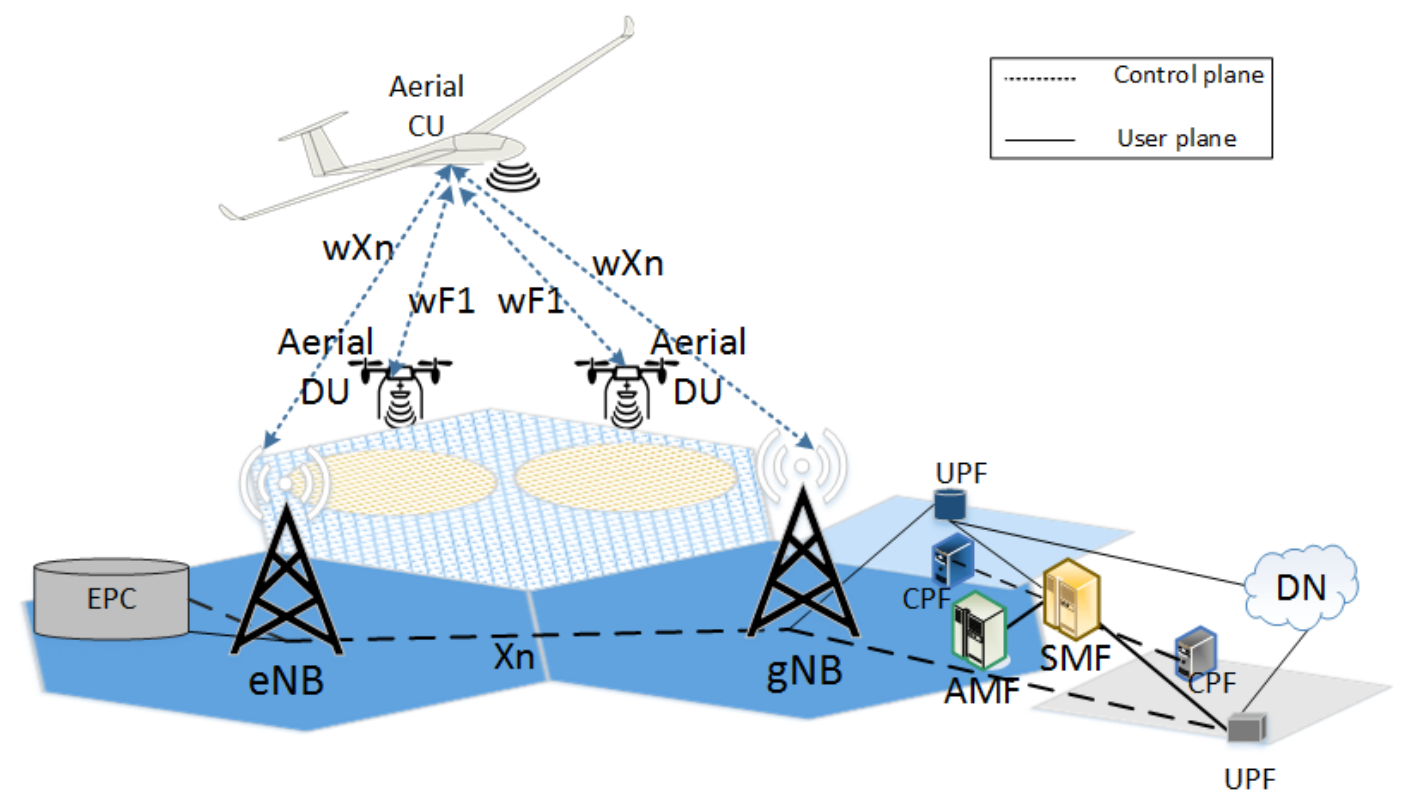

Figure 5.7: Architecture option for WIDs where a fleet of WIDs with aerial-DUs and an aerial-CU with a specific 5G-core-slice for WID-originated traffic (blue slice) is utilized. Interfaces with potential enhancements are marked with "w".

differences between option-2 and option-3 are the split of the control-plane and the split level of the user-plane. In option-3, both signaling and data radio bearers are divided at the 


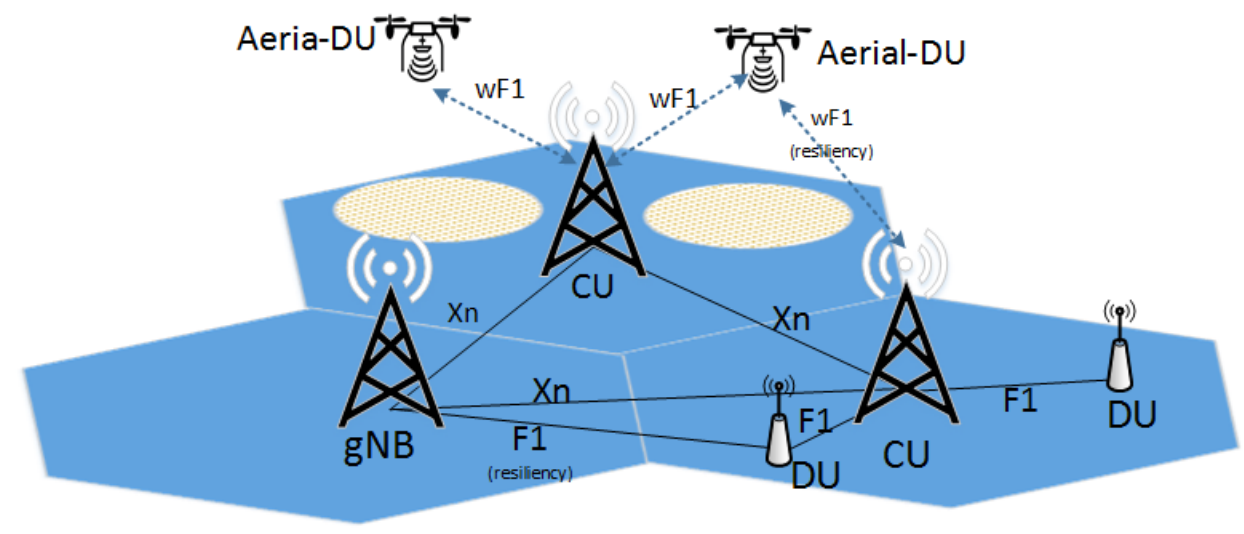

Figure 5.8: Architecture option for WIDs where Aerial-DUs associated with terrestrialCUs (no specific 5G-core-slice for WID-originated traffic) is utilized. Interfaces with potential enhancements are marked with "w".

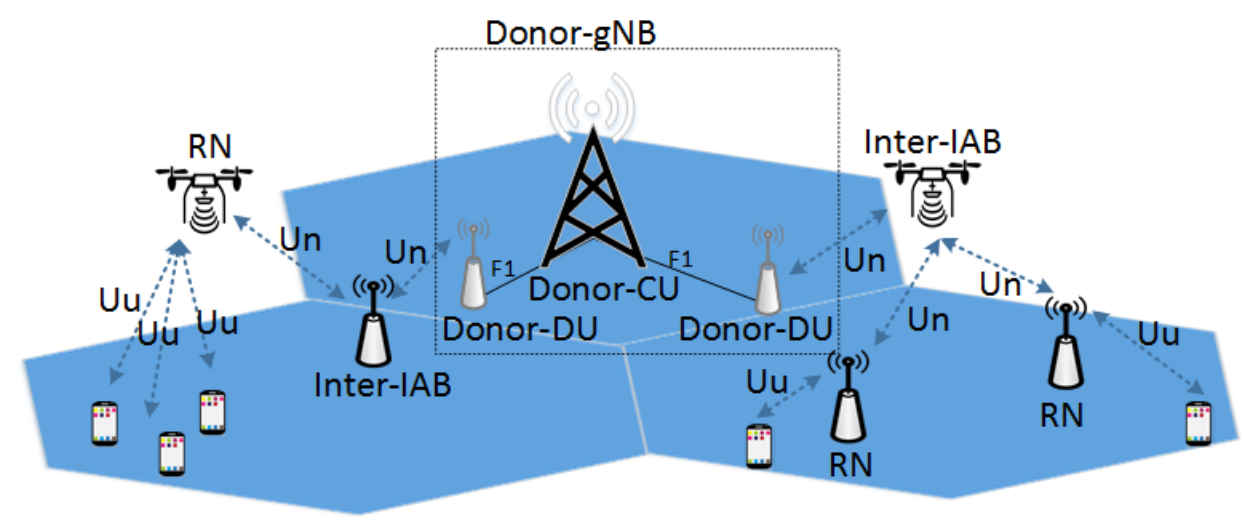

Figure 5.9: Architecture option for WIDs where WIDs are utilized as relay nodes and intermediate IAB nodes. There are at least protocol stack differences between the two types of nodes.

RLC level, where high-RLC is with CU and low-RLC is with DU. Option-3 thus enables lighter DUs compared to option-2. However, option-3 wastes fronthaul bandwidth for radio resource control and management protocols, as observed from procedures in TS 38.401.

RAN1 studies lower-layer-split options in TR 38.816. Based on calculations with parameter sets including uplink/downlink channel bandwidth, modulation scheme, and number of antenna ports, Option-6 and Option-7.1 require 4.1 to $18.2 \mathrm{Gbps}$, and 37.6 to 454.6 Gbps fronthaul rate for downlink, respectively. Despite the large variation of required fronthaul rate, note that achieving high rates between aerial-DUs and CUs are possible thanks 
to LOS opportunities, and wide spectrum in NR.

There are multiple trade-offs when split options are considered for WIDs. First, lowerlayer-split increases bandwidth requirement and decreases tolerance to latency for the fronthaul link, compared to upper layer splits. It also increases the complexity to transmit the signal over the fronthaul (especially for PHY layer). Nonetheless, lower-layer-split reduces computational requirements of DU significantly. That can increase airtime, if fronthaul links with high-SINR and large bandwidth are available. Finally, lower-layer-split makes centralization more effective, and increases the number of UEs that can be served by the DU.

\subsubsection{Integrated Access and Backhaul}

While users expect increased service capabilities from 5G, operators have their own expectations, such as scalability of the network economically. Recently, RAN WGs approved study items for "Integrated Access and Backhaul" (IAB) to support wireless network densification via reducing the need to scale the transport network for dense deployments. The study item proposes methods to utilize IAB by taking advantage of the large bandwith available in NR, and native deployment of multi-beam systems and massive MIMO. IAB is generally considered for relays (rTRP), similar to LTE-A relay nodes (RN). Although support for mobile relays is left for future releases, the study has fundamental importance for WIDs in two ways: First, an aerial relay can utilize wireless backhauling that is natively supported by $5 \mathrm{G}$ networks. Second, a WID as an intermediate IAB node can reduce number of hops, and provide topology flexibility thanks to LOS and mobility.

For the IAB study in 3GPP TR 38.874, the primary responsible working group is RAN2, but RAN1 and RAN3 are listed as other responsible groups, e.g., RAN1 is responsible for physical layer enhancements for reliable transmission with high spectral efficiency. TR 38.874 considers frequencies up to $100 \mathrm{GHz}$, leaving visible light communications out of scope but including mmWave. Split options similar to CU/DU can be used for L2/L3 RNs, e.g., option-2- and option-3-like splits (Sec. 5.2.3). The support for above-6GHz frequencies require topology adaption, i.e., autonomously reconfiguring the backhaul network due to blockage, e.g., seasonal changes, new buildings. Adaptation is also required to manage changes in the demand, e.g., local congestion. In addition, multi-hop backhauling 
is required to cope with the short range of above- $6 \mathrm{GHz}$ frequencies. Multi-connectivity is another critical solution, where either an rTRP is connected with multiple donor-nodes, and switches fast between them based on the channel conditions, or UE is connected with multiple rTRPs using the same backhaul nodes [233]. A potential solution for rTRPs is revisiting L2- and L3-relay architectures, and investigating their trade-offs in the IAB context. For instance, there are proposals to use RLC-based relaying with an adaptation layer carrying routing address, and QoS related information [233]. Finally, IAB supports half duplex (TDM/FDM/SDM), but does not support full duplex studies in RAN1 yet. Especially for in-band backhaul with respect to access link, half-duplexing constraints will be supported. Out-band backhauling too will be supported, however, additional RAN features may be needed. For DC, rTRPs will support EN-DC in both non-standalone and SA deployments.

Although Rel-15 considers fixed relays, it does not preclude optimization for mobile relays in future releases. Regarding above- $6 \mathrm{GHz}$ frequencies, WIDs provide more degrees of freedom due to mobility, which relaxes frequent switching, and multi-connectivity requirements. Also, WIDs' mobility enables flexibility in topology design, and alleviates the problem of coverage holes by following the crowd at the cell-edge. Since LOS is likely, subtle enhancements in PHY layer may suffice. However, the limiting factor, especially for in-band backhauling, is co-channel interference [40]. Re-visiting L2/L3-relay architectures, and investigating their trade-offs in drone-IAB context can reveal practical solutions, e.g., utilizing RLC-based relaying with an adaptation layer carrying routing address, drone's coordinates, and QoS related information [233].

\subsubsection{Licensed/Unlicensed options for MEDs and WIDs}

When operating in a leased (licensed) band, mobile network operators enjoy continuous availability of the dedicated radio resources and mainly optimize performance under intraoperator co-channel interference. However, such licensed spectrum resources are scarce and expensive to retain. With the ever-increasing traffic load, exploiting the vast and freeof-charge unlicensed spectrum has been receiving tremendous interest, especially in $5 \mathrm{GHz}$ and $60 \mathrm{GHz}$ bands. Unlike operations in licensed spectrum, region and band-specific unlicensed spectrum regulations govern many aspects of channel access and physical transmission parameters to allow for coexistence with other operator networks/RATs such as 
incumbent WLAN networks. Among these regulations are the listen-before-talk mechanism, maximum channel occupancy time, maximum transmit EIRP, maximum PSD, and minimum occupational bandwidth. Such regulations made it possible only for low power nodes (small cells) to gain access to the unlicensed spectrum similar to WLAN devices.

Given the specifications of licensed-assisted access (LAA) to unlicensed spectrum (Rel13 TS 36.213 to Rel-15 TS 37.213), an interesting question arises: Can drones benefit from LAA? Since only small cells can exploit such mechanisms due to the maximum transmit power regulations, the answer actually depends on the envisioned range and altitude of the drone application compared to the coverage of these LAA cells, which may not extend beyond $150 \mathrm{~m}$. In Rel-15, a study item on NR-based access to unlicensed spectrum (NR-U) was approved with the objective of porting the NR enhancements, such as the flexible numerology (sub-carrier spacing), mini-slot, frame structure, and wideband operations, to the unlicensed spectrum either below or above $7 \mathrm{GHz}$ (TS 38.889). Employing larger sub-carrier spacing and/or mini-slot based transmissions increases throughput, but renders less energy per symbol for the same transmit power, and hence, reduced coverage. Consequently, it is more difficult to support the access links of some drone applications.

While mmWave and beamforming techniques of NR can enhance the received SINR at drones via narrow beams and interference suppression, they may not enhance the received signal power in the unlicensed band as a transmit power backoff would be necessary to comply with the maximum $\mathrm{EIRP}^{7}$ regulations. This in fact suggests that a $5 \mathrm{G}$ NR-U air interface, may not be suitable for the radio access links of NR-U MEDs as they would have to maintain close proximity to the serving micro/pico cells. Contrarily, it can be suitable for the radio access links of NR-U UEs served by non-standalone WIDs. In Fig. 5.2 and Fig. 5.3 the serving drone-secondary-gNB (drone-SgNB) can maintain close proximity to the DC UEs while its user-plane/control-plane connections are provided over the licensed band within the large coverage of a Macro Master-eNB (MeNB) or Master-gNB (MgNB), respectively.

The operation of the UEs in Fig. 5.2 should be supported by the specified EN-DC options-3/3A where a wireless $\mathrm{Xx}-\mathrm{C}$ connection to the MeNB carries the control-plane data. First, a wireless Xx-U connection carries the user-plane data through the LTE PDCP layer to the NR RLC layer of the drone-SgNB, if a split bearer between the MeNB and

\footnotetext{
${ }^{7}$ EIRP stands for equivalent isotropic radiated power.
} 
the drone-SgNB (option-3) is utilized. Second, when EPC switches to a secondary cell group (SCG) bearer (option-3A), wireless S1-U connection to the EPC carries the userplane data directly through the NR PDCP layer of the drone-SgNB. These two cases are indeed of particular importance since they are likely to resemble the early stage of migration from LTE to 5G. Similarly, operations of UEs in Fig. 5.3 should be supported by specified full-5G options-4/4A where a wireless $\mathrm{Xn}-\mathrm{C}$ connection to the Master-gNB carries the control-plane data. In case of a split bearer between the Master-gNB and the drone-SgNB (option-4), a wireless Xn-U connection carries the user-plane data through the NR SDAP that is required for mapping bearer packets to QoS classes before the PDCP layer. Whereas in case of a (switched) SCG bearer (option-4A), a wireless N3 connection to the 5G-core carries the user-plane data directly through the NR SDAP sublayer of the drone-SgNB.

Mobile network access to the unlicensed spectrum, either through LTE LAA or 5G NR-U, could have been designed to fulfill only the regulatory requirements. However, a fair coexistence with incumbent RATs such as WiFi would not be guaranteed. Due to their inherent fairness to WiFi, older technologies specified before LAA for LTE to exploit the unlicensed spectrum through Carrier WiFi offloading are still credible technologies, and thus expected to be adopted in 5G networks as shown in Fig. 5.4 and Fig. 5.5. One solution thereof is aggregation of NR and WLAN at the PDCP-level (NWA). In such case, only a non-collocated implementation is feasible for a drone-WLAN termination. This is obviously the simplest and most cost-efficient integration scenario of WIDs for unlicensed access. The other solution is IP-level integration of NR and WLAN (NWIP) wherein UEs operate in multi-homing mode and handle two different IP flows over the two air interfaces. However, the IP flow offloaded to the drone-access-point (drone-AP) is relatively unsecured and an IPsec tunnel is therefore established between the Master-gNB and the UE through the drone-AP by encapsulating the NR PDUs using an NWIPEP sublayer.

As described, despite its limitations for MEDs especially above $7 \mathrm{GHz}$, NR-Unlicensed as well as the NR-WLAN aggregation/integration options provide multifarious integration options for WIDs while exploiting the vast and free-of-charge unlicensed spectrum. Further studies should investigate the efficiency of these options by considering multitude scenarios from dense deployments to IoT and URLLC. 


\subsection{G Core}

In order to better exploit advantages of NFV and SDN, enable automation, and improve flexibility, 5G-core networks established a service-based architecture (Fig. 5.1(b)). Principles of the 5G-core architecture design includes allowing a NF to talk to other NFs directly, supporting multi-vendor integration, and allowing different slices with unique configurations (TS 23.501).

Service-based 5G-core enhances existing functions by dissecting their functionalities into new functions. For instance, the mobility and management entity (MME) of EPC is dissected into AMF and SMF (Fig. 5.1(b)). AMF is the termination of non-access stratum, and includes functionalities such as mobility management, access authentication, and lawful intercept (TS 23.501). Similarly, SMF has roaming functionality of MME, and also control-plane functionalities of Service- and packet-gateways, such as UE IP address allocation and management. Most control-plane NFs are enhanced with slice-awareness, e.g., AMF selects network slice during attachment of UEs (TS 38.801, Fig. 5.1(a)). Single network slice selection assistance information (S-NSSAI) identifies a network slice. An SNSSAI consists of a slice/service type (SST) referring to main characteristics of NS, e.g., eMBB, uRLLC, and a slice differentiator (SD) that differentiates the slice within the same SST optionally.

Increased support for virtualization and slicing at core makes it easier to integrate new functions, specifically supporting MEDs and WIDs [2]. However, there are many issues on how to use this flexibility. Since MED traffic varies from latency-tolerant telemetry data, to bandwidth hungry live video streaming, MEDs may be associated with multiple slices. A UE can be associated with 8 slice instances simultaneously, and whether it is enough for MEDs or not depends on the application, and design of the slices. TS 23.401 recently included subscription support for high-level aerial-UE functions, and how they are transmitted in the EPC during handover. However, slicing aspects of MED support, e.g., a specific SST or SD value, is missing. Network exposure function (NEF) allows exposure of control-plane NF capabilities in a controlled fashion to external entities, e.g., untrusted application function, edge computing, and other vendor's control-plane NFs. NEF can make deploying edge computing to support MED operations ${ }^{8}$, more economic and faster.

\footnotetext{
8"Hangar and Vapor IO to deliver autonomous robotics at the kinetic edge," Mar 2018, Accessed: 2019-08-26. [Online]. Available: https://www.vapor.io/
} 
Regarding WIDs, a key issue is carrying their traffic without impacting the existing services. One way is to create a slice for a WID, potentially with some shared controlplane NFs and non-shared user-plane functions. However, creating only one slice may not be enough, since there may be UEs with different services. Then, many trade-offs appear in this scenario: Since drone-BS operations are expensive, the objective is to utilize drone-BS for as many services as possible. Therefore, multiple 5G-core slices may need to be created for each WID. That is costly, hard to manage, and increases the burden on wireless interfaces for updates regarding NSSAIs, e.g., N2 and N1. If existing slices are shared, providing sufficient isolation is challenging; monitoring traffic of many services require high-level of granularity. Complexity increases exponentially for integration of drone fleets, which necessitates efficient management systems.

\subsection{G Network Management}

Network management is traditionally responsible for FCAPS, i.e., Fault, Charging, Authorization, Performance, and Security management. Recently, challenging management requirements of flexible networks lead to new management functions, and a service-based management and orchestration system. NSI, NSSI, and NF Management Service Functions (NSMF, NSSMF, and NFMF, respectively) consist of other services, e.g., performance management (PM). In this architecture, a management service provider and its consumer can have various relationships as shown in Fig. 5.10(a).

Accordingly, drones can be managed by various management entities, depending on the drone's role. Fig. 5.10(b) show an example slice provisioning procedure, where drones are shown as a slice consumer, as well as NSSI, NF, and infrastructure providers. In fact, TS 28.530 supports Network-slice-as-a-Service (NSaaS), which may be delivered with different information and management exposure. However, NF and infrastructure providers are not explicitly supported by current standards.

In the presence of various business options, and a layered network with constituents at different levels, integrating WIDs may require updating TSs. For instance, new network resource model entities, similar to the additions in TS 28.541 for CU/DU, may be hangar-and-vapor-to-deliver-autonomous-robotics-at-the-kinetic-edge/. 


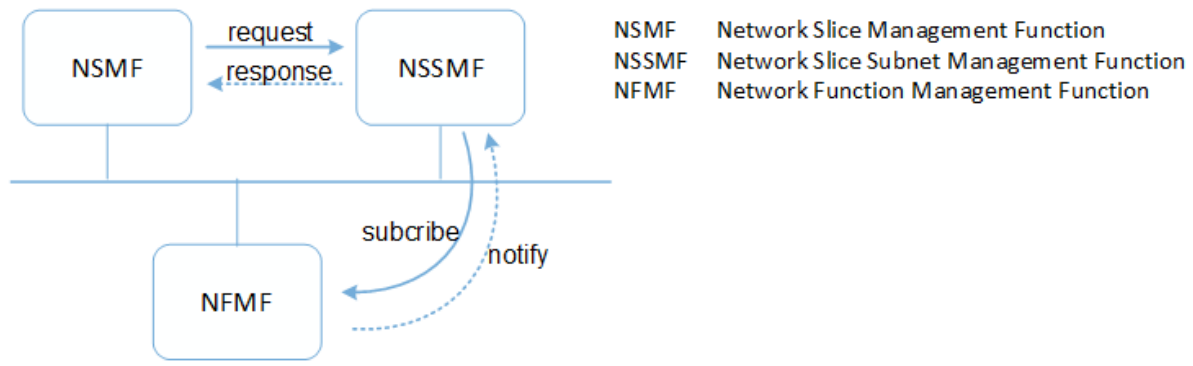

(a)

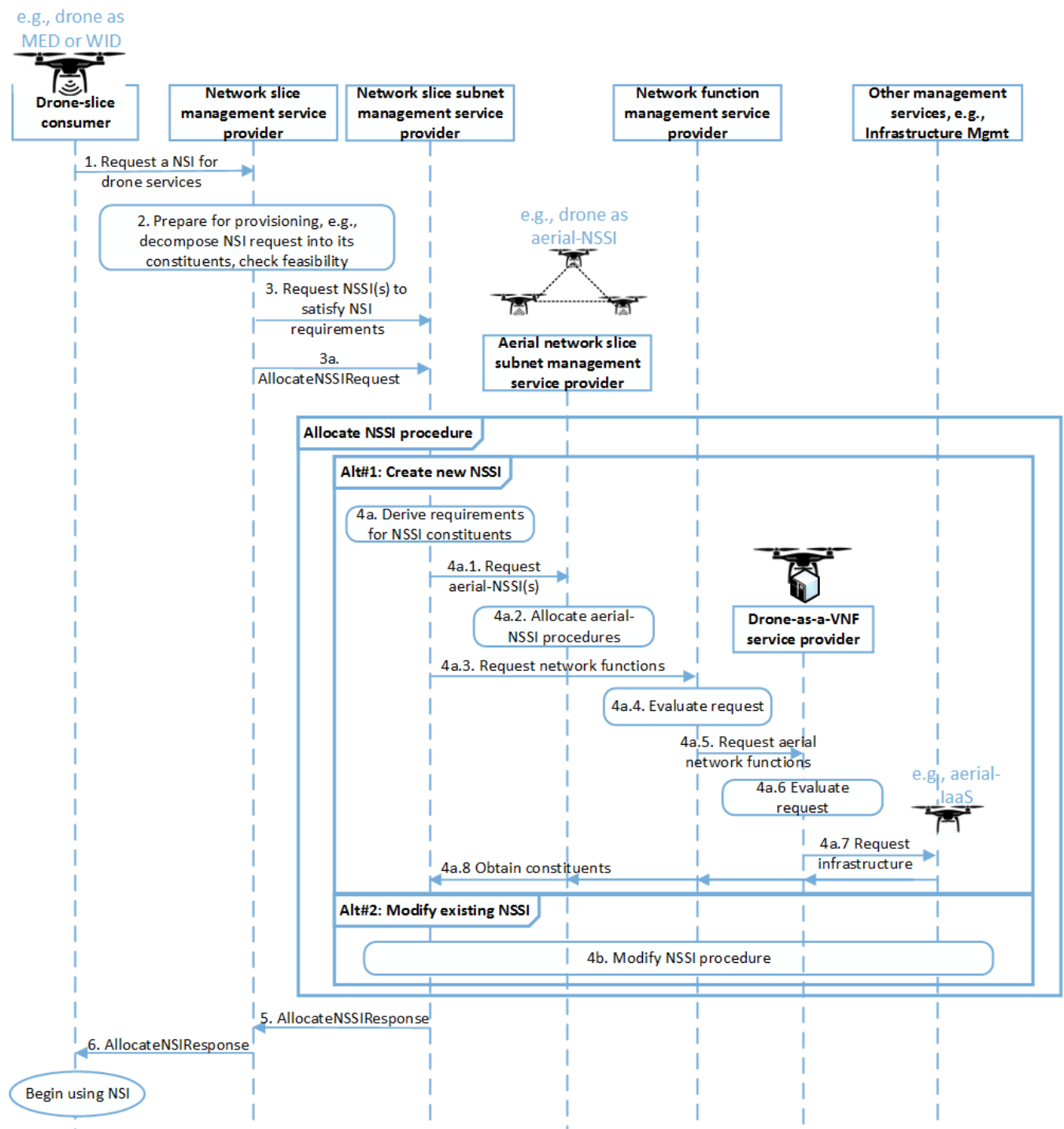

(b)

Figure 5.10: Network management system overview: (a) SBA allows any network management service provider to access another via "request-response" or "subscribenotify" messages; (b) Overview of network slice provisioning procedures: An NSI request may be responded via creation of a new slice or modification of an existing one. Provisioning requests are decomposed into their constituents by corresponding management service providers. Drones can have varying roles in mobile networks, e.g., as slice consumer, slice provider, and slice constituent. 
useful. Moreover, FCAPS requirements for WIDs are not yet investigated in detail. For instance, how to configure a WID to measure and report load information? Are there additional alarms that should be raised by WIDs or MEDs, e.g., remaining fuel, malfunction? Moreover, there are proposals to turn network management systems into the core of the network automation and optimization with end-to-end data collection and analysis capability [234]. That makes network management critical in drone integration as the provider of information to make decisions, e.g., determining role of WIDs. However, these automation mechanisms are not yet in the standards, and there is no consensus on distribution of responsibilities, e.g., between SA2 and SA5. In fact, solutions to these issues can only be obtained by clear descriptions of roles of WIDs in 3GPP networks, and coordination among standardization organizations and working groups. Issues requiring more than coordination but substantial research are discussed next.

\subsection{Future of Standardization and Research Directions}

Although Rel-15 constitutes a rather early phase in $5 \mathrm{G}$ standardization to focus on specific solutions for drone operations, the incomplete study items in Rel-15 along with those in Rel-16 hint directions for future of standardization. Improvements of fundamental procedures (e.g., aerial-UE registration) for MEDs, definition of new terminal types, study on isolation of network resources for drones from security and performance aspects can be listed among standardization and research topics. Selected topics are discussed in detail next.

\subsubsection{Network Configuration and Slice Design for WIDs}

Traditionally, existing resources (e.g., infrastructure, transport network) are considered during network configuration and slice design. Meaning that the set of RAN nodes and NFs are pre-determined. However, versatility of WIDs adds degrees of freedom, and requires new design methods and configuration strategies:

1. RAN NSS design: An architectural role for WIDs (Sec. 5.2.3) that best fits the network need and situation must be selected. For instance, if the terrestrial nodes are 
eNBs, CU/DU is not an option; AR can be preferred. That requires WIDs that are capable of handling all control plane and establishing reliable Xn links with eNBs. If $\mathrm{gNBs}$ and $5 \mathrm{G}$ UEs are majority, WID can be deployed as a DU. In that case, split option must be determined based on fronthaul connection capability, technical specifications of the WID, and the need of the network (e.g., alleviating congestion, or increasing coverage). Donor-nodes, reliable topologies, and resource allocation strategies (e.g., hard slice, soft slice, updating RRMs) must be configured appropriately.

2. CN NSS design: Impact of integrating WIDs on 5GC must be minimized by utilizing flexible networking techniques while satisfying communication requirements, such as security, isolation, and latency. For that purpose, modifications on existing CN NSS, e.g., initializing new NFs, or increasing capacity of existing resources can be performed. TN capacity must be assessed, and additional capacity should be allocated, if needed. If WID-integration-slice is a newly created slice, AMF, NSSF etc. should be re-configured and new UPFs are created. SMF may be pre-configured with UPF selection for reducing latency. Moreover, MEC functions to provide additional computation for WID/MED operations may be deployed strategically. Finally, all NFs should be configured to perform any additional measurements that are required for maintaining WID traffic.

Automated design to satisfy network requirements is key to agile networking. In addition to technical issues of design, business roles for WIDs are discussed next.

\subsubsection{Support For New Business Models}

Business models for MEDs are similar to those for UEs. For instance, the control link of an MED may be a high-priority link, such that in case of congestion, specific resources may be reserved for them to prevent outage. Ultimately, MED cases are likely to be supported via evolutionary standardization, configuration and application methods.

On the contrary, business models for WIDs can be complicated and diverse (Fig 5.10(b)):

- Aerial-IaaS: This applies when WID acts as a single node, that is similar to a NF. 
WIDs can serve as access points, or VNFs, i.e., drone-as- $a$-VNF. For instance, when MME is malfunctioned, congested, or more reliability is needed, a drone with MME functionality can be utilized. For applications requiring low-latency, a drone can be utilized to bring application function closer by drone-as-MEC. In cases depicted in Fig. 5.6 through Fig. 5.9 and Fig. 5.2 through Fig. 5.5, a WID is used as an access point in an appropriate architectural role.

- Aerial-NSSaaS: Multiple WIDs can form an aerial network slice subnet (A-NSS), e.g., overlaying an existing RAN-NSS or 5GC-NSS (e.g., UPFs, MMEs, and MEC applications).

Assuming an operator obtains WID services from a service provider (SP), a trade-off occurs between the operators aiming not to expose information, and SPs aiming not to delegate management capabilities. For instance, if a SP manages an A-NSS, then the operator needs to expose information, such as service types/requirements and user contexts, to the SP for fine-tuning management, e.g., PM. While diverse business models increase flexibility of WID services, and reduce the stress on operators, exposure of information and management capabilities become critical issues.

\subsubsection{New QoS and KPI Parameters}

There will likely be a need for new KPI and QoS parameters for MEDs and WIDs. For instance, since WIDs are deployed on-demand, their services should generating enough revenue. Although the number of served users can be a nominal KPI parameter, considering more complex charging schemes, it may be inadequate to determine profitability of WID operations. Hence a new KPI, profitability of WID, can be considered, which would involve charging policies, operation cost, and number of served users in formulation. We propose integration efficiency as an umbrella-KPI considering 5GC impact (e.g., additional load and signaling), RAN impact (e.g., resource allocation, interference), performance degradation for UEs in the cells of donor-nodes (e.g., due to interference, or scarcity of resources), topology efficiency (e.g., number of hops, reliability). KPIs for drones will need to be merged with new KPIs, for which some examples can be found in [235]. 


\subsection{Summary and Discussions}

We investigated practicality of integrating drones into modern wireless networks by surveying 5G Phase-I standardization from radio access networks to system and architecture aspects. Our end-to-end study shows that future wireless networks are promising in terms of providing efficient mechanisms and required network flexibility for both serving drones and benefiting from them. Despite these capabilities, we also observe that there is no study in $5 \mathrm{G}$ standardization for efficient integration of aerial nodes. Hopefully this fundamental open issue will be addressed by collaboration from the industry and academia in the future releases of 5G (Release-17 the earliest) and beyond 5G networks. 


\section{Chapter 6}

\section{Conclusions and Future Work}

\subsection{Summary and Conclusions}

In this thesis, we addressed placement, channel modeling, and integration of drone-BSs. In particular, we proposed an efficient 3-D placement method, in which a drone-BS is positioned with respect to locations of users. Since the proposed 3-D placement method determines both horizontal and vertical position of the drone-BS, the size of the coverage area is also obtained simultaneously. Afterwards, we applied a similar placement method for mmWave-drone-BSs by considering a channel model taking human body blockage into account.

To further refine positioning of drone-BSs, we investigate air-to-ground channel models. In particular, we propose a channel model for urban areas, where the map of the area is known. Our model enables utilizing the information obtained from city maps for more accurate estimations of average path loss compared to a widely used channel model. We show that the proposed channel model increases accuracy of drone-BS operations, e.g., number of drone-BSs to be used to cover an area, via simulation results and ray-tracing simulations.

We introduced spatial network configuration, where users receive incentives to move towards the coverage area of a drone-BS. SNC is a unique framework due to the interaction between user and access point mobility. We proposed three different methods for SNC based on that interaction, namely, uncoordinated, joint, and semi-joint SNC. For 
uncoordinated-SNC, first the drone-BS is positioned, and then incentives are sent to users that are in proximity to the coverage area. For joint and semi-joint SNC, the drone-BS is positioned by considering potential users that are in proximity to the prospected coverage area. We show that joint and semi-joint SNC increases profitability of the network significantly in comparison to uncoordinated SNC. We also show that semi-joint SNC is several orders of magnitude more computationally efficient than joint SNC, while providing similar profitability.

Finally, we investigate integration of drone-BSs into $5 \mathrm{G}$ and beyond wireless networks from a system-level perspective. First, after discussing drone types, their opportunities and challenges, we propose a drone management framework. We examine how state-ofthe-art technologies, such as NFV and SDN can be used to integrate drone-BSs in future networks with multi-tenancy. We further examine NFV and SDN by using virtual wireless networking, which makes NFV and SDN exploit drone-BS mobility more efficiently. Our simulation results show that the integration method and underlying technology utilization is case-specific, i.e., depends on the improvement demanded by the network. Furthermore, we take a look into the contemporary and prospective wireless networks from a standardization perspective to find out whether $5 \mathrm{G}$ and beyond wireless networks are suitable to benefit from drone-BSs and support drone-type users. Our comprehensive study shows that fundamental networking technologies for drones are being employed, however, there is still a long way towards true drone-BS integration.

\subsection{Future Work}

This thesis is prepared when drone-BS concept and their interactions with terrestrial networks was being re-discovered after being abandoned for decades. The main reasons for that considerable come-back can be summarized as the technical developments making drones more affordable and capable at the same time, and problems due to (so far) inevitable densification of wireless networks. Hence, one of the main contributions of this thesis is investigating the interactions of drone-BSs and terrestrial networks with a holistic view, and identifying several key research directions. The second contribution of this study is attempting to fill the gaps in some of those identified areas: 3D drone-BS positioning, air-to-ground channel modelling, exploiting user and access point mobility jointly, tailoring 
virtualization methods to benefit from drone-BS mobility, and drone-BS integration. Similarly, our future work is also twofold: First, we identify a key research direction, which is integrated ground-air-space communication (IGASC) networks. IGASC networks include not only drone-BSs, but also satellites and high-altitude platforms, which results in a multi-layered heterogeneous aerial network that continuously interacts with the underlying terrestrial networks. Then, we plan to investigate methods to obtain seamless and reliable operation of IGASC networks as follows.

\subsubsection{User Involved IGASC}

In Ch. 3 we only scratched the surface of user involvement in wireless networks. Moreover, user involvement is lightly discussed in the literature as well, especially for wireless network users [153]. On the other hand, wireless networks are considered as an enabler of cyber-physical systems, rather than being cyber-physical systems themselves. The broad perspective of this line of research is to understand implications of such a perception change for wireless networks. Following can be thought as potential research directions.

- Incentive design for wireless network users and applications: Purpose of giving incentives is an integral part of their design. The studies on designing incentives for the purpose of wireless network performance improvement, such as [192], is very rare in the literature. Cross-disciplinary studies involving fields such as psychology, consumer behaviour, and marketing can be conducted to determine the incentives that are likely to be accepted by the users. In fact, one of the future work extending Ch. 3 is comparing incentives that designed by cross-disciplinary methods and incentives that are learned by using artificial intelligence based methods [236-238]. Furthermore, the requests from the users should be tailored based on the needs of wireless applications. It is another investigation to see if the incentives should be altered based on the need of the network. For instance, we need to examine if different incentives should be designed to delay the demand versus change the position of the user. Accordingly, in Ch. 3 we only investigated spatial requests from the user. In fact, user involvement can be multifaceted and include temporal, contextual and spatial requests simultaneously. To the best of our knowledge, such complex incentive design does not exist for wireless networks. 
- Drone-in-the-loop: In Ch. 4, we discussed drones as WIDs, which can provide services such as drone-as-a-service, network-function-as-a-service, and network-sliceas-a-service [106]. In a fully automated and self-adapting IGASC, WIDs can be individual agents providing a portfolio of services. In such a scenario, wireless connectivity providers will need to make intelligent offers to these agents. These offers would consider a multitude of factors such as characteristics of the demanded service, the value of the service, and the location. The agents, either automatically or with human-intervention, can choose the best offers. The performance of the wireless network would then constitute from many local decision, i.e., the management and optimization of the network is distributed. Investigating performance of these distributed systems from the perspective of WID involvement is one of our future works.

- Spatiotemporal UIL for satellite services: There are unique opportunities for spatial UIL, because satellites and HAPS can have deterministic schedules. That allows the network operator to plan for a demand distribution and try to realize it as much as possible by persuading users to delay their demand based on the arrival time of the satellite beams and/or change their location based on the satellite beams if they are highly mobile users.

\subsubsection{Self-Adapting and Autonomous IGASC Networks}

The recent developments in wireless networks and wireless network management shows a tendency to automate networks as much as possible, to undprecedented degrees as discussed in 5. Ambitious work items from 3GPP and ETSI, such as [234], aim at minimizing human intervention and maximising self-adaptation capabilities of wireless networks. Our study in Ch. 4 and Ch. 5 about integration of drone-BSs lays the foundation of our future studies on wireless network automation. The following are some of the key issues that we hope to address.

- Equalitarian and secure data collection: Automation of wireless networks requires granular data to be collected. That raises several issues regarding security of data collection and storage: First, data security is important to protect both individual and provider information. Making data anonymous while keeping its relevancy is one 
of the fundamental issues. Another issue is making equalitarian distribution of data, such that none of the network or content providers, vendors, individuals or other actors creates a monopoly. Creating secure and liberal data storage and retrieval pools is a challenge to be addressed to ensure monopolies are prevented. We are planning to investigate blockchain methods to develop efficient measures for data collection by extending our integration framework in Ch. 4 with consideration to the latest 5G developments in 5 [239, 240].

- Autonomous decision making: IGASC networks are more complex than existing terrestrial networks because a high number of wireless nodes will be mobile. For instance, the SpaceX satellite constellation may consist of 12000 LEO satellites to provide wireless backhaul to $5 \mathrm{G}$ and beyond wireless networks [241]. The resulting IGASC will require precise coordination among aerial, terrestrial and space nodes, as well as robustness against malfunction. Note that space nodes can be beyond simple relays by utilizing content caching and various RATs. It means that a simple coordination will not be enough to fully utilize IGASC. Rather, dynamic decisions are necessary. Considering high reliability requirements for next generation wireless networks, timely human intervention in case of malfunction or selections such as which content to cache, or which RAT to utilize may not be possible. Therefore, automated decision making is an inevitable characteristic of future wireless networks. More specifically, we are planning to work on routing, energy efficient drone-BS placement, and service node selection problems. Furthermore, we would like to extend our study in Sec. 2.3 in order to automatically use the intelligence derived from city maps and perform smart 3-D placement of drone-BSs.

- Agile networking: We are planning to extend our study in Ch. 4 on VWA to include satellites and HAPS. Such complex scenarios require big data processing and fast converging AI-based computation methods. Therefore, we begin by investigating data sources and artificial data generation methods. Then, we compare AI methods and develop an efficient AI framework to utilize agile networking as enabled by virtualization and slicing. 


\subsubsection{IoT in IGASC}

IGASC networks should enable vast IoT services. For instance, millions of sensors and actuators of smart cities may rely on wireless connectivity provided by IGASC networks. Moreover, IGASC can enable cost-efficient coverage for off-grid IoT devices. However, both IoT and IGASC nodes are energy-limited and efficient communications is an extremely important aspect. As discussed in Ch. 1, NOMA, and mmWave with beamforming can be enablers of efficient IoT-IGASC networks. Therefore, we are planning to enhance our study in Section 2.2 to include NOMA and beamforming, such as the studies in [91] and [242]. Differently from the study in [91], we plan to consider blockage and mmWave frequencies. Also, we would like to investigate more dynamic beamforming schemes due to the mobility of mmWave-drone-BSs.

\subsubsection{Channel modelling for IGASC}

We only scratched the surface of channel modelling for drone-BSs in Sec. 2.3. In addition to the challenges of terrestrial channel models, air-to-ground channel modelling suffers from variety of devices, altitudes and environments. Slowly but surely, the literature is developing and several air-to-ground channel models are being proposed [8]. Among the proposed models, our study in [29] represents a rather unique area where channel models are based on city map information. In Sec. 2.3, we assumed a rather simplistic city where buildings, block sizes and street widths, as well as their orientation is artificially regular. However, cities in real life consist of irregular street widths, orientation, building shapes and heights. Therefore, a natural extension of Sec. 2.3 is improving the model to reflect those irregularities. There are two main approaches to achieve an improved model: First, extensive measurement campaigns can be performed to make measurements and derive correlations between city map layouts and radio signal. While that is the most accurate and fundamental channel modelling study, it is also expensive, requires extensive collaborations, and takes a very long process. An alternative method is utilizing two powerful computation tools: Combining ray tracing simulations and artificial intelligence methods, we may be able to obtain accurate air-to-ground channel models with less measurements. In other words, it may be possible to develop adaptive models, instead of static ones. Hence, the need for extensive measurement campaigns can be alleviated. Our future work is based 
on the former method.

All in all, the future of this field is certainly bright and sky is not the limit anymore. 


\section{List of References}

[1] M. Kamel, W. Hamouda, and A. Youssef, "Ultra-dense networks: A survey," IEEE Communications Surveys E Tutorials, vol. 18, pp. 2522-2545, May 2016.

[2] I. Bor-Yaliniz and H. Yanikomeroglu, "The new frontier in RAN heterogeneity: Multi-tier drone-cells," IEEE Commun. Mag., vol. 54, pp. 48-55, Nov. 2016.

[3] H. Shakhatreh, A. H. Sawalmeh, A. Al-Fuqaha, Z. Dou, E. Almaita, I. Khalil, N. S. Othman, A. Khreishah, and M. Guizani, "Unmanned aerial vehicles (UAVs): A survey on civil applications and key research challenges," IEEE Access, vol. 7, pp. 48572-48634, Apr. 2019.

[4] A. Otto, N. Agatz, J. Campbell, B. Golden, and E. Pesch, "Optimization approaches for civil applications of unmanned aerial vehicles (UAVs) or aerial drones: A survey," Networks, vol. 72, pp. 411-458, Mar. 2018.

[5] A. Fotouhi, H. Qiang, M. Ding, M. Hassan, L. G. Giordano, A. Garcia-Rodriguez, and J. Yuan, "Survey on UAV cellular communications: Practical aspects, standardization advancements, regulation, and security challenges," IEEE Communications Surveys $\mathcal{E}$ Tutorials, Mar. 2019. Early Access.

[6] M. Mozaffari, W. Saad, M. Bennis, Y.-H. Nam, and M. Debbah, "A tutorial on UAVs for wireless networks: Applications, challenges, and open problems," IEEE Communications Surveys $\mathcal{E}$ Tutorials, vol. 21, pp. 2334-2360, Mar. 2019.

[7] S. Hayat, E. Yanmaz, and R. Muzaffar, "Survey on unmanned aerial vehicle networks for civil applications: A communications viewpoint," IEEE Communications Surveys $\mathcal{E}$ Tutorials, vol. 18, pp. 2624-2661, Apr. 2016.

[8] W. Khawaja, I. Güvenç, D. W. Matolak, U. Fiebig, and N. Schneckenburger, "A survey of air-to-ground propagation channel modeling for unmanned aerial vehicles," IEEE Communications Surveys $\mathcal{E}$ Tutorials, vol. 21, pp. 2361-2391, May 2019.

[9] C. Yan, L. Fu, J. Zhang, and J. Wang, "A comprehensive survey on UAV communication channel modeling," IEEE Access, vol. 7, pp. 107769-107792, Aug. 2019.

[10] D. W. Matolak and R. Sun, "Air-ground channel characterization for unmanned aircraft systems - Part I: Methods, measurements, and models for over-water settings," IEEE Transactions on Vehicular Technology, vol. 66, pp. 26-44, Jan. 2017. 
[11] R. Sun and D. W. Matolak, "Air-ground channel characterization for unmanned aircraft systems - Part II: Hilly and mountainous settings," IEEE Transactions on Vehicular Technology, vol. 66, pp. 1913-1925, Mar. 2017.

[12] D. W. Matolak and R. Sun, "Air-ground channel characterization for unmanned aircraft systems - Part III: The suburban and near-urban environments," IEEE Transactions on Vehicular Technology, vol. 66, pp. 6607-6618, Aug. 2017.

[13] R. Sun, D. W. Matolak, and W. Rayess, "Air-ground channel characterization for unmanned aircraft systems - Part IV: Airframe shadowing," IEEE Transactions on Vehicular Technology, vol. 66, pp. 7643-7652, Sept. 2017.

[14] H. Jiang, Z. Zhang, L. Wu, and J. Dang, "Three-dimensional geometry-based UAVMIMO channel modeling for A2G communication environments," IEEE Communications Letters, vol. 22, pp. 1438-1441, July 2018.

[15] H. Chang, J. Bian, C. Wang, Z. Bail, J. Sun, and X. Gao, "A 3D wideband geometrybased stochastic model for UAV air-to-ground channels," in IEEE Global Coтmunications Conference (GLOBECOM), pp. 206-212, Dec. 2018.

[16] H. Chang, J. Bian, C. Wang, Z. Bai, W. Zhou, and E. M. Aggoune, "A 3D nonstationary wideband GBSM for low-altitude UAV-to-ground V2V MIMO channels," IEEE Access, vol. 7, pp. 70719-70732, May 2019.

[17] X. Cheng and Y. Li, "A 3-D geometry-based stochastic model for UAV-MIMO wideband nonstationary channels," IEEE Internet of Things Journal, vol. 6, pp. 16541662, Apr. 2019.

[18] Y. Xu, X. Xia, K. Xu, and Y. Wang, "Three-dimension massive MIMO for air-toground transmission: Location-assisted precoding and impact of AoD uncertainty," IEEE Access, vol. 5, pp. 15582-15596, July 2017.

[19] Y. Shi, R. Enami, J. Wensowitch, and J. Camp, "UABeam: UAV-based beamforming system analysis with in-field air-to-ground channels," in 15th Annual IEEE International Conference on Sensing, Communication, and Networking (SECON), pp. 1-9, June 2018.

[20] T. J. Willink, C. C. Squires, G. W. K. Colman, and M. T. Muccio, "Measurement and characterization of low-altitude air-to-ground MIMO channels," IEEE Transactions on Vehicular Technology, vol. 65, pp. 2637-2648, Apr. 2016.

[21] C. Zhang, L. Ma, and K. Pang, "Stable A/G MIMO transmission aided by openloop calibrated channel estimation and adaptive precoding," IEEE Transactions on Vehicular Technology, vol. 65, pp. 8806-8811, Oct. 2016.

[22] X. Cai, N. Wang, J. Rodrguez-Pieiro, X. Yin, A. P. Yuste, W. Fan, G. Zhang, G. F. Pedersen, and L. Tian, "Low altitude air-to-ground channel characterization in LTE network," in 13th European Conference on Antennas and Propagation (EuCAP), pp. 1-5, Mar. 2019.

[23] R. Amorim, H. Nguyen, P. Mogensen, I. Z. Kovcs, J. Wigard, and T. B. Srensen, "Radio channel modeling for UAV communication over cellular networks," IEEE Wireless Communications Letters, vol. 6, pp. 514-517, Aug. 2017. 
[24] P. Ladosz, H. Oh, G. Zheng, and W. Chen, "A hybrid approach of learning and model-based channel prediction for communication relay UAVs in dynamic urban environments," IEEE Robotics and Automation Letters, vol. 4, pp. 2370-2377, July 2019.

[25] Q. Feng, J. McGeehan, E. K. Tameh, and A. R. Nix, "Path loss models for air-toground radio channels in urban environments," in IEEE 63rd Vehicular Technology Conference, vol. 6, pp. 2901-2905, May 2006.

[26] F. Ono, K. Takizawa, H. Tsuji, and R. Miura, "S-band radio propagation characteristics in urban environment for unmanned aircraft systems," in International Symposium on Antennas and Propagation (ISAP), pp. 1-4, Nov. 2015.

[27] A. Al-Hourani, S. Kandeepan, and A. Jamalipour, "Modeling air-to-ground path loss for low altitude platforms in urban environments," in Proc. IEEE Global Communications Conference (GLOBECOM), pp. 2898-2904, Dec. 2014.

[28] A. Al-Hourani, S. Kandeepan, and S. Lardner, "Optimal LAP altitude for maximum coverage,” IEEE Wireless Communication Letters, vol. 3, pp. 569-572, Dec. 2014.

[29] I. Bor-Yaliniz, S. S. Szyszkowicz, and H. Yanikomeroglu, "Environment-aware drone-base-station placements in modern metropolitans," IEEE Wireless Communications Letters, vol. 7, pp. 372-375, June 2017.

[30] E. Greenberg and P. Levy, "Channel characteristics of UAV to ground links over multipath urban environments," in IEEE International Conference on Microwaves, Antennas, Communications and Electronic Systems (COMCAS), pp. 1-4, Nov. 2017.

[31] R. I. Bor-Yaliniz, A. El-Keyi, and H. Yanikomeroglu, "Efficient 3-D placement of an aerial base station in next generation cellular networks," in IEEE International Conference on Communications (ICC), pp. 1-5, July 2016.

[32] M. Alzenad, A. El-Keyi, F. Lagum, and H. Yanikomeroglu, "3D placement of an unmanned aerial vehicle base station (UAV-BS) for energy-efficient maximal coverage," IEEE Wireless Communications Letters, vol. 6, pp. 434-437, Aug. 2017.

[33] M. Alzenad, A. El-Keyi, and H. Yanikomeroglu, "3-D placement of an unmanned aerial vehicle base station for maximum coverage of users with different QoS requirements," IEEE Wireless Communications Letters, vol. 7, pp. 38-41, Feb. 2018.

[34] H. Huang, A. V. Savkin, M. Ding, and M. A. Kaafar, "Optimized deployment of drone base station to improve user experience in cellular networks," Journal of Network and Computer Applications, vol. 144, pp. 49-58, July 2019.

[35] E. Kalantari, H. Yanikomeroglu, and A. Yongacoglu, "On the number and 3D placement of drone base stations in wireless cellular networks," in IEEE Vehicular Technology Conference, pp. 1-6, Sept. 2016.

[36] W. Shi, J. Li, W. Xu, H. Zhou, N. Zhang, S. Zhang, and X. Shen, "Multiple dronecell deployment analyses and optimization in drone assisted radio access networks," IEEE Access, vol. 6, pp. 12518-12529, Feb. 2018. 
[37] C. T. Cicek, H. Gultekin, B. Tavli, and H. Yanikomeroglu, "UAV base station location optimization for next generation wireless networks: Overview and future research directions," in 1st International Conference on Unmanned Vehicle SystemsOman (UVS), pp. 1-6, Feb. 2019.

[38] S. Sekander, H. Tabassum, and E. Hossain, "Multi-tier drone architecture for 5G/B5G cellular networks: Challenges, trends, and prospects," IEEE Communications Magazine, vol. 56, pp. 96-103, Mar. 2018.

[39] M. Mozaffari, A. Taleb Zadeh Kasgari, W. Saad, M. Bennis, and M. Debbah, "Beyond 5G with UAVs: Foundations of a 3D wireless cellular network," IEEE Transactions on Wireless Communications, vol. 18, pp. 357-372, Jan. 2019.

[40] E. Kalantari, I. Bor-Yaliniz, A. Yongacoglu, and H. Yanikomeroglu, "User association and bandwidth allocation for terrestrial and aerial base stations with backhaul considerations," in IEEE 28th Annual International Symposium on Personal, Indoor, and Mobile Radio Communications (PIMRC), pp. 1-6, Oct. 2017.

[41] R. Ghanavi, E. Kalantari, M. Sabbaghian, H. Yanikomeroglu, and A. Yongacoglu, "Efficient 3D aerial base station placement considering users mobility by reinforcement learning," in IEEE Wireless Communications and Networking Conference (WCNC), pp. 1-6, Apr. 2018.

[42] F. Lagum, I. Bor-Yaliniz, and H. Yanikomeroglu, "Strategic densification with UAVBSs in cellular networks," IEEE Wireless Communications Letters, vol. 7, pp. 384387, June 2018.

[43] Y. Zeng, X. Xu, and R. Zhang, "Trajectory design for completion time minimization in UAV-enabled multicasting," IEEE Transactions on Wireless Communications, vol. 17, pp. 2233-2246, Apr. 2018.

[44] A. French, M. Mozaffari, A. Eldosouky, and W. Saad, "Environment-aware deployment of wireless drones base stations with Google Earth simulator," in IEEE International Conference on Pervasive Computing and Communications Workshops (PerCom Workshops), pp. 868-873, Mar. 2019.

[45] J. Lyu, Y. Zeng, and R. Zhang, "UAV-aided offloading for cellular hotspot," IEEE Transactions on Wireless Communications, vol. 17, pp. 3988-4001, June 2018.

[46] A. Merwaday and I. Guvenc, "UAV assisted heterogeneous networks for public safety communications," in IEEE Wireless Communications and Networking Conference Workshops (WCNCW), pp. 329-334, Mar. 2015.

[47] I. Bor-Yaliniz, A. El-Keyi, and H. Yanikomeroglu, "Spatial configuration of agile wireless networks with Drone-BSs and user-in-the-loop," IEEE Transactions on Wireless Communications, vol. 18, pp. 753-768, Feb. 2019.

[48] H. Ghazzai, M. B. Ghorbel, A. Kadri, and M. J. Hossain, "Energy efficient 3D positioning of micro unmanned aerial vehicles for underlay cognitive radio systems," in IEEE International Conference on Communications (ICC), pp. 1-6, May 2017. 
[49] W. Shi, J. Li, W. Xu, H. Zhou, N. Zhang, and X. Shen, "3D drone-cell deployment optimization for drone assisted radio access networks," in IEEE/CIC International Conference on Communications in China (ICCC), pp. 1-6, Oct. 2017.

[50] J. Plachy, Z. Becvar, P. Mach, R. Marik, and M. Vondra, "Joint positioning of flying base stations and association of users: Evolutionary-based approach," IEEE Access, vol. 7, pp. 11454-11463, Jan. 2019.

[51] M. Mozaffari, W. Saad, M. Bennis, and M. Debbah, "Optimal transport theory for cell association in UAV-enabled cellular networks," IEEE Communications Letters, vol. 21, pp. 2053-2056, Sept. 2017.

[52] M. Mozaffari, W. Saad, M. Bennis, and M. Debbah, "Mobile internet of things: Can UAVs provide an energy-efficient mobile architecture?," in IEEE Global Communications Conference (GLOBECOM), pp. 1-6, Dec. 2016.

[53] M. Mozaffari, W. Saad, M. Bennis, and M. Debbah, "Mobile unmanned aerial vehicles (UAVs) for energy-efficient internet of things communications," IEEE Trans. Wireless Commun., vol. 16, pp. 7574-7589, Nov. 2017.

[54] Q. Wu, Y. Zeng, and R. Zhang, "Joint trajectory and communication design for multi-UAV enabled wireless networks," IEEE Transactions on Wireless Communications, vol. 17, pp. 2109-2121, Mar. 2018.

[55] J. Lyu, Y. Zeng, R. Zhang, and T. J. Lim, "Placement optimization of UAV-mounted mobile base stations," IEEE Communications Letters, vol. 21, pp. 604-607, Mar. 2017.

[56] U. Challita, W. Saad, and C. Bettstetter, "Deep reinforcement learning for interference-aware path planning of cellular-connected UAVs," in IEEE International Conference on Communications (ICC), pp. 1-7, IEEE, May 2018.

[57] M. Chen, M. Mozaffari, W. Saad, C. Yin, M. Debbah, and C. S. Hong, "Caching in the sky: Proactive deployment of cache-enabled unmanned aerial vehicles for optimized quality-of-experience," IEEE Journal on Selected Areas in Communications, vol. 35, pp. 1046-1061, May 2017.

[58] P. Yang, X. Cao, C. Yin, Z. Xiao, X. Xi, and D. Wu, "Proactive drone-cell deployment: Overload relief for a cellular network under flash crowd traffic," IEEE Transactions on Intelligent Transportation Systems, vol. 18, pp. 2877-2892, Oct. 2017.

[59] A. Fotouhi, M. Ding, and M. Hassan, "Dynamic base station repositioning to improve performance of drone small cells," in IEEE Globecom Workshops (GC Wkshps), pp. 1-6, Dec. 2016.

[60] E. Koyuncu, M. Shabanighazikelayeh, and H. Seferoglu, "Deployment and trajectory optimization of UAVs: A quantization theory approach," IEEE Transactions on Wireless Communications, vol. 17, pp. 8531-8546, Dec. 2018.

[61] N. Rupasinghe, A. S. Ibrahim, and I. Güvenç, "Optimum hovering locations with angular domain user separation for cooperative UAV networks," in IEEE Global Communications Conference (GLOBECOM), pp. 1-6, Dec. 2016. 
[62] A. Merwaday, A. Tuncer, A. Kumbhar, and I. Güvenç, "Improved throughput coverage in natural disasters: Unmanned aerial base stations for public-safety communications," IEEE Vehicular Technology Magazine, vol. 11, pp. 53-60, Dec. 2016.

[63] M. Mozaffari, W. Saad, M. Bennis, and M. Debbah, "Efficient deployment of multiple unmanned aerial vehicles for optimal wireless coverage," IEEE Communications Letters, vol. 20, pp. 1647-1650, Aug. 2016.

[64] J.-W. Cho, S. Kim, and J.-H. Kim, "Multi-UAV placements to minimize power consumption in urban environment," in International Conference on Ubiquitous Information Management and Communication, pp. 306-314, Springer, May 2019.

[65] A. Akarsu and T. Girici, "Fairness aware multiple drone base station deployment," IET Communications, vol. 12, pp. 425-431, Mar. 2017.

[66] S. Yan, M. Peng, and X. Cao, "A game theory approach for joint access selection and resource allocation in UAV assisted IoT communication networks," IEEE Internet of Things Journal, vol. 6, pp. 1663-1674, Apr. 2019.

[67] C. Pan, J. Yi, C. Yin, J. Yu, and X. Li, "Joint 3D UAV placement and resource allocation in software-defined cellular networks with wireless backhaul," IEEE Access, vol. 7, pp. 104279-104293, July 2019.

[68] H. Ghazzai, M. Ben Ghorbel, A. Kadri, M. J. Hossain, and H. Menouar, "Energyefficient management of unmanned aerial vehicles for underlay cognitive radio systems," IEEE Transactions on Green Communications and Networking, vol. 1, pp. 434-443, Dec. 2017.

[69] J. Lu, S. Wan, X. Chen, and P. Fan, "Energy-efficient 3D UAV-BS placement versus mobile users' density and circuit power," in IEEE Globecom Workshops (GC Wkshps), pp. 1-6, Dec. 2017.

[70] L. Sboui, H. Ghazzai, Z. Rezki, and M. Alouini, "Energy-efficient power allocation for UAV cognitive radio systems," in IEEE 86th Vehicular Technology Conference (VTC-Fall), pp. 1-5, Sept. 2017.

[71] H. El Hammouti, M. Benjillali, B. Shihada, and M. Alouini, "Learn-as-you-fly: A distributed algorithm for joint 3D placement and user association in multi-UAVs networks," IEEE Transactions on Wireless Communications, Sept. 2019. Early Access.

[72] Y. Sun, T. Wang, and S. Wang, "Location optimization and user association for unmanned aerial vehicles assisted mobile networks," IEEE Transactions on Vehicular Technology, vol. 68, pp. 10056-10065, Oct. 2019.

[73] X. Sun and N. Ansari, "Jointly optimizing drone-mounted base station placement and user association in heterogeneous networks," in IEEE International Conference on Communications (ICC), pp. 1-6, May 2018.

[74] M. Chen, W. Saad, and C. Yin, "Liquid state machine learning for resource allocation in a network of cache-enabled LTE-U UAVs," in IEEE Global Communications Conference (GLOBECOM), pp. 1-6, Dec. 2017. 
[75] A. Azizi, S. Parsaeefard, M. R. Javan, N. Mokari, and H. Yanikomeroglu, "Profit maximization in 5G+ networks with heterogeneous aerial and ground base stations," IEEE Transactions on Mobile Computing, pp. 1-1, July 2019. Early Access.

[76] H. Hellaoui, O. Bekkouche, M. Bagaa, and T. Taleb, "Aerial control system for spectrum efficiency in UAV-to-cellular communications," IEEE Communications Magazine, vol. 56, pp. 108-113, Oct. 2018.

[77] Y. Pang, Y. Zhang, Y. Gu, M. Pan, Z. Han, and P. Li, "Efficient data collection for wireless rechargeable sensor clusters in harsh terrains using UAVs," in IEEE Global Communications Conference, pp. 234-239, Dec. 2014.

[78] S. H. Alsamhi, O. Ma, M. S. Ansari, and F. A. Almalki, "Survey on collaborative smart drones and Internet of Things for improving smartness of smart cities," IEEE Access, vol. 7, pp. 128125-128152, Aug. 2019.

[79] A. Ahmed, M. Awais, T. Akram, S. Kulac, M. Alhussein, and K. Aurangzeb, "Joint placement and device association of UAV base stations in IoT networks," Sensors, vol. 19, p. 2157, May 2019.

[80] X. Zhou, S. Durrani, J. Guo, and H. Yanikomeroglu, "Underlay drone cell for temporary events: Impact of drone height and aerial channel environments," IEEE Internet of Things Journal, vol. 6, pp. 1704-1718, Apr. 2019.

[81] W. Feng, J. Wang, Y. Chen, X. Wang, N. Ge, and J. Lu, "UAV-aided MIMO communications for 5G internet of things," IEEE Internet of Things Journal, vol. 6, pp. 1731-1740, Apr. 2019.

[82] L. Xie, J. Xu, and R. Zhang, "Throughput maximization for UAV-enabled wireless powered communication networks," IEEE Internet of Things Journal, vol. 6, pp. 1690-1703, Apr. 2019.

[83] J. Wang, C. Jiang, Z. Wei, C. Pan, H. Zhang, and Y. Ren, "Joint UAV hovering altitude and power control for space-air-ground IoT networks," IEEE Internet of Things Journal, vol. 6, pp. 1741-1753, Apr. 2019.

[84] X. Liu, Z. Li, N. Zhao, W. Meng, G. Gui, Y. Chen, and F. Adachi, "Transceiver design and multihop D2D for UAV IoT coverage in disasters," IEEE Internet of Things Journal, vol. 6, pp. 1803-1815, Apr. 2019.

[85] D. Ebrahimi, S. Sharafeddine, P. Ho, and C. Assi, "UAV-aided projection-based compressive data gathering in wireless sensor networks," IEEE Internet of Things Journal, vol. 6, pp. 1893-1905, Apr. 2019.

[86] E. Tuyishimire, A. Bagula, S. Rekhis, and N. Boudriga, "Cooperative data muling from ground sensors to base stations using UAVs," in IEEE Symposium on Computers and Communications (ISCC), pp. 35-41, July 2017.

[87] N. Rupasinghe, Y. Yapıcı, I. Güvenç, and Y. Kakishima, "Non-orthogonal multiple access for mmWave drones with multi-antenna transmission," in 51st Asilomar Conference on Signals, Systems, and Computers, pp. 958-963, Oct. 2017. 
[88] S. K. Zaidi, S. F. Hasan, X. Gui, N. Siddique, and S. Ahmad, "Exploiting UAV as NOMA based relay for coverage extension," in 2nd International Conference on Computer Applications Information Security (ICCAIS), pp. 1-5, May 2019.

[89] N. Rupasinghe, Y. Yapıcı, I. Güvenç, and Y. Kakishima, "Comparison of limited feedback schemes for NOMA transmission in mmWave drone networks," in IEEE 19th International Workshop on Signal Processing Advances in Wireless Communications (SPAWC), pp. 1-5, June 2018.

[90] M. F. Sohail, C. Y. Leow, and S. Won, "Non-orthogonal multiple access for unmanned aerial vehicle assisted communication," IEEE Access, vol. 6, pp. 2271622727, Apr. 2018.

[91] L. Liu, S. Zhang, and R. Zhang, "Exploiting NOMA for multi-beam UAV communication in cellular uplink," in IEEE International Conference on Communications (ICC), pp. 1-6, May 2019.

[92] X. Liu, J. Wang, N. Zhao, Y. Chen, S. Zhang, Z. Ding, and F. R. Yu, "Placement and power allocation for NOMA-UAV networks," IEEE Wireless Communications Letters, vol. 8, pp. 965-968, June 2019.

[93] P. K. Sharma and D. I. Kim, "UAV-enabled downlink wireless system with nonorthogonal multiple access," in IEEE Globecom Workshops (GC Wkshps), pp. 1-6, Dec. 2017.

[94] T. M. Nguyen, W. Ajib, and C. Assi, "A novel cooperative NOMA for designing UAV-assisted wireless backhaul networks," IEEE Journal on Selected Areas in Communications, vol. 36, pp. 2497-2507, Nov. 2018.

[95] X. Pang, Z. Li, X. Chen, Y. Cao, N. Zhao, Y. Chen, and Z. Ding, "UAV-aided NOMA networks with optimization of trajectory and precoding," in 10th International Conference on Wireless Communications and Signal Processing (WCSP), pp. 1-6, Oct. 2018.

[96] N. Rupasinghe, Y. Yapıc1, I. Güvenç, H. Dai, and A. Bhuyan, "Enhancing physical layer security for NOMA transmission in mmWave drone networks," in 52nd Asilomar Conference on Signals, Systems, and Computers, pp. 729-733, Oct. 2018.

[97] I. Ahmad, S. Shahabuddin, T. Kumar, J. Okwuibe, A. Gurtov, and M. Ylianttila, "Security for 5G and beyond," IEEE Communications Surveys $\mathcal{E}$ Tutorials, May 2019. Early Access.

[98] Y. Chen and L. Wang, "Privacy protection for internet of drones: A network coding approach," IEEE Internet of Things Journal, vol. 6, pp. 1719-1730, Apr. 2019.

[99] O. Galinina, L. Militano, S. Andreev, A. Pyattaev, K. Johnsson, A. Orsino, G. Araniti, A. Iera, M. Dohler, and Y. Koucheryavy, "Demystifying competition and cooperation dynamics of the aerial mmwave access market," arXiv preprint arXiv:1608.04943, 2016.

[100] F. Cheng, D. Zou, J. Liu, J. Wang, and N. Zhao, "Learning-based user association for dual-UAV enabled wireless networks with D2D connections," IEEE Access, vol. 7, pp. 30672-30682, Mar. 2019. 
[101] E. Christy, R. P. Astuti, B. Syihabuddin, B. Narottama, O. Rhesa, and F. Rachmawati, "Optimum UAV flying path for device-to-device communications in disaster area," in International Conference on Signals and Systems (ICSigSys), pp. 318-322, May 2017.

[102] D. Liu, J. Wang, Y. Xu, Y. Zhang, Q. Wu, and A. Anpalagan, "Opportunistic data ferrying in UAV-assisted D2D networks: A dynamic hierarchical game," in IEEE International Conference on Communications (ICC), pp. 1-6, May 2019.

[103] M. Mozaffari, W. Saad, M. Bennis, and M. Debbah, "Unmanned aerial vehicle with underlaid device-to-device communications: Performance and tradeoffs," IEEE Transactions on Wireless Communications, vol. 15, pp. 3949-3963, June 2016.

[104] X. Li, D. Guo, H. Yin, and G. Wei, "Drone-assisted public safety wireless broadband network," in IEEE Wireless Communications and Networking Conference Workshops (WCNCW), pp. 323-328, Mar. 2015.

[105] A. Alsharoa, H. Ghazzai, A. Kadri, and A. E. Kamal, "Spatial and temporal management of cellular HetNets with multiple solar powered drones," IEEE Transactions on Mobile Computing, Feb. 2019. Early Access.

[106] I. Bor-Yaliniz, M. Salem, G. Senerath, and H. Yanikomeroglu, "Is 5G ready for drones: A look into contemporary and prospective wireless networks from a standardization perspective," IEEE Wireless Communications, vol. 26, pp. 18-27, Feb. 2019.

[107] L. Wang, H. Yang, J. Long, K. Wu, and J. Chen, "Enabling ultra-dense UAV-aided network with overlapped spectrum sharing: Potential and approaches," IEEE Network, vol. 32, pp. 85-91, Sept. 2018.

[108] Z. T. Dydek, A. M. Annaswamy, and E. Lavretsky, "Adaptive control of quadrotor UAVs: A design trade study with flight evaluations," IEEE Transactions on Control Systems Technology, vol. 21, no. 4, pp. 1400-1406, 2013.

[109] Y. Li and S. Song, "A survey of control algorithms for quadrotor unmanned helicopter," in 2012 IEEE International Conference on Advanced Computational Intelligence (ICACI), pp. 365-369, 2012.

[110] P. E. Pounds, D. R. Bersak, and A. M. Dollar, "Stability of small-scale UAV helicopters and quadrotors with added payload mass under PID control," Autonomous Robots, vol. 33, no. 1-2, pp. 129-142, 2012.

[111] "Professional aerial and ground imaging." https://www.dji.com/ca/ products/professional?site=brandsite\&from=nav\#integrated-system. Accessed: 2020-06-06.

[112] V. Sharma, K. Srinivasan, H.-C. Chao, K.-L. Hua, and W.-H. Cheng, "Intelligent deployment of UAVs in $5 \mathrm{G}$ heterogeneous communication environment for improved coverage," Journal of Network and Computer Applications, pp. 94-105, May 2017. 
[113] S. Park, H. Kim, K. Kim, and H. Kim, "Drone formation algorithm on 3D space for a drone-based network infrastructure," in IEEE 27th Annual International Symposium on Personal, Indoor, and Mobile Radio Communications (PIMRC), pp. 1-6, Sept. 2016.

[114] C. Zhang and W. Zhang, "Spectrum sharing for drone networks," IEEE Journal on Selected Areas in Communications, vol. 35, pp. 136-144, Jan. 2017.

[115] J. Košmerl and A. Vilhar, "Base stations placement optimization in wireless networks for emergency communications," in IEEE International Conference on Communications Workshops (ICC), pp. 200-205, June 2014.

[116] A. M. Hayajneh, S. A. R. Zaidi, D. C. McLernon, and M. Ghogho, "Drone empowered small cellular disaster recovery networks for resilient smart cities," in IEEE International Conference on Sensing, Communication and Networking (SECON Workshops), pp. 1-6, Dec. 2016.

[117] M. Narang, W. Liu, J. Gutierrez, and L. Chiaraviglio, "A cyber physical buses-anddrones mobile edge infrastructure for large scale disaster emergency communications," in IEEE 37th International Conference on Distributed Computing Systems Workshops (ICDCSW), pp. 53-60, June 2017.

[118] A. Fotouhi, M. Ding, and M. Hassan, "Dynamic base station repositioning to improve performance of drone small cells," in IEEE Globecom Workshops (GC Wkshps), pp. 1-6, Dec. 2016.

[119] Y. Zeng and R. Zhang, "Energy-efficient UAV communication with trajectory optimization," IEEE Transactions on Wireless Communications, vol. 16, pp. 3747-3760, June 2017.

[120] O. Andryeyev and A. Mitschele-Thiel, "Increasing the cellular network capacity using self-organized aerial base stations," in Proceedings of the 3rd Workshop on Micro Aerial Vehicle Networks, Systems, and Applications, pp. 37-42, ACM, June 2017.

[121] S. A. W. Shah, T. Khattab, M. Z. Shakir, and M. O. Hasna, "Association of networked flying platforms with small cells for network centric 5G+C-RAN," in IEEE 28th Annual International Symposium on Personal, Indoor, and Mobile Radio Communications (PIMRC), pp. 1-7, Oct. 2017.

[122] Z. Becvar, M. Vondra, P. Mach, J. Plachy, and D. Gesbert, "Performance of mobile networks with UAVs: Can flying base stations substitute ultra-dense small cells?," in 23th European Wireless Conference (European Wireless 2017), pp. 1-7, May 2017.

[123] J. Lyu, Y. Zeng, and R. Zhang, "Spectrum sharing and cyclical multiple access in UAV-aided cellular offloading," in IEEE Global Communications Conference (GLOBECOM), pp. 1-6, Dec. 2017.

[124] C. Rametta and G. Schembra, "Designing a softwarized network deployed on a fleet of drones for rural zone monitoring," Future Internet, vol. 9, p. 8, Mar. 2017. 
[125] P. L. Mehta and R. Prasad, "Aerial-heterogeneous network: A case study analysis on the network performance under heavy user accumulations," Wireless Personal Communications, vol. 94, pp. 1-20, May 2017.

[126] S. Kandeepan, K. Gomez, L. Reynaud, and T. Rasheed, "Aerial-terrestrial communications: Terrestrial cooperation and energy-efficient transmissions to aerial base stations," IEEE Transactions on Aerospace and Electronic Systems, vol. 50, pp. 27152735, Oct. 2014.

[127] P. Zhan, K. Yu, and A. L. Swindlehurst, "Wireless relay communications with unmanned aerial vehicles: Performance and optimization," IEEE Transactions on Aerospace and Electronic Systems, vol. 47, pp. 2068-2085, July 2011.

[128] S. Rohde, M. Putzke, and C. Wietfeld, "Ad hoc self-healing of OFDMA networks using UAV-based relays,” Ad Hoc Networks, vol. 11, pp. 1893-1906, Sept. 2013.

[129] "Propagation data and prediction methods required for the design of terrestrial broadband radio access systems operating in a frequency range from 3 to $60 \mathrm{GHz}$," Recommendation ITU-R P.1410-5, International Telecommunication Union Radiocommunication Sector (ITU-R), Feb. 2012.

[130] M. Mirahsan, R. Schoenen, and H. Yanikomeroglu, "HetHetNets: Heterogeneous traffic distribution in heterogeneous wireless cellular networks," IEEE Journal on selected areas in communications, vol. 33, pp. 2252-2265, Oct. 2015.

[131] Z. Xiao, H. Dong, L. Bai, D. O. Wu, and X. Xia, "Unmanned aerial vehicle base station (UAV-BS) deployment with millimeter-wave beamforming," IEEE Internet of Things Journal, vol. 7, pp. 1336-1349, Feb. 2020.

[132] Z. Xiao, P. Xia, and X. Xia, "Enabling UAV cellular with millimeter-wave communication: Potentials and approaches," IEEE Communications Magazine, vol. 54, pp. 66-73, May 2016.

[133] C. Zhang, W. Zhang, W. Wang, L. Yang, and W. Zhang, "Research challenges and opportunities of UAV millimeter-wave communications," IEEE Wireless Communications, vol. 26, pp. 58-62, Feb. 2019.

[134] J. G. Andrews, S. Buzzi, W. Choi, S. V. Hanly, A. Lozano, A. C. Soong, and J. C. Zhang, "What will 5G be?," IEEE Journal on selected areas in communications, vol. 32, pp. 1065-1082, June 2014.

[135] M. Abouelseoud and G. Charlton, "The effect of human blockage on the performance of millimeter-wave access link for outdoor coverage," in IEEE 77th Vehicular Technology Conference (VTC Spring),, pp. 1-5, June 2013.

[136] M. Gapeyenko, A. Samuylov, M. Gerasimenko, D. Moltchanov, S. Singh, M. R. Akdeniz, E. Aryafar, N. Himayat, S. Andreev, and Y. Koucheryavy, "On the temporal effects of mobile blockers in urban millimeter-wave cellular scenarios," IEEE Transactions on Vehicular Technology, vol. 66, pp. 10124-10138, Nov. 2017. 
[137] S. Sun, T. S. Rappaport, T. A. Thomas, A. Ghosh, H. C. Nguyen, I. Z. Kovács, I. Rodriguez, O. Koymen, and A. Partyka, "Investigation of prediction accuracy, sensitivity, and parameter stability of large-scale propagation path loss models for 5G wireless communications," IEEE Transactions on Vehicular Technology, vol. 65, pp. 2843-2860, May 2016.

[138] "Study on channel model for frequencies from 0.5 to $100 \mathrm{GHz}$ (Release 14)." 3GPP RAN, 2018. TR 38.901 V14.3.0.

[139] M. R. Akdeniz, Y. Liu, M. K. Samimi, S. Sun, S. Rangan, T. S. Rappaport, and E. Erkip, "Millimeter wave channel modeling and cellular capacity evaluation," IEEE Journal on Selected Areas in Communications, vol. 32, pp. 1164-1179, June 2014.

[140] S. Hur, T. Kim, D. J. Love, J. V. Krogmeier, T. A. Thomas, and A. Ghosh, "Millimeter wave beamforming for wireless backhaul and access in small cell networks," IEEE Transactions on Communications, vol. 61, pp. 4391-4403, Oct. 2013.

[141] M. Mozaffari, W. Saad, M. Bennis, and M. Debbah, "Drone small cells in the clouds: Design, deployment and performance analysis," in IEEE Global Communications Conference (GLOBECOM), pp. 1-6, Dec. 2015.

[142] M. Gapeyenko, A. Samuylov, M. Gerasimenko, D. Moltchanov, S. Singh, E. Aryafar, S.-P. Yeh, N. Himayat, S. Andreev, and Y. Koucheryavy, "Analysis of humanbody blockage in urban millimeter-wave cellular communications," in IEEE International Conference on Communications (ICC), pp. 1-7, May 2016.

[143] Y. Zeng, R. Zhang, and T. J. Lim, "Wireless communications with unmanned aerial vehicles: Opportunities and challenges," IEEE Communications Magazine, vol. 54, pp. 36-42, May 2016.

[144] J. G. Andrews, T. Bai, M. N. Kulkarni, A. Alkhateeb, A. K. Gupta, and R. W. Heath, "Modeling and analyzing millimeter wave cellular systems," IEEE Trans. on Commun., vol. 65, pp. 403-430, Oct. 2017.

[145] "MOSEK ApS optimization software." https://WWw.mosek.com/. Accessed: 2020-02-18.

[146] H. Shakhatreh, A. Khreishah, and B. Ji, "Providing wireless coverage to highrise buildings using UAVs," in IEEE International Conference on Communications (ICC), pp. 1-7, May 2016.

[147] J. Lyu, Y. Zeng, R. Zhang, and T. J. Lim, "Placement optimization of UAV-mounted mobile base stations," IEEE Commun. Lett., vol. 21, pp. 604-607, Mar. 2017.

[148] "Propagation data and prediction methods for the planning of short-range outdoor radiocommunication systems and radio local area networks in the frequency range $300 \mathrm{MHz}$ to $100 \mathrm{GHz}$," Rec. P series, Radio propagation, Radio Communication Sector of International Telecommunication Union (ITU-R), July 2015.

[149] "Cities ranked by number of $150 \mathrm{~m}+$ completed buildings." https://WwW . skyscrapercenter. com/cities. Accessed: 2020-02-18. 
[150] 3GPP TR 22.862, "Study on 3D channel model for LTE," 2017. v. 14.0.0.

[151] M. Simunek, F. P. Fontn, and P. Pechac, "The UAV low elevation propagation channel in urban areas: Statistical analysis and time-series generator," IEEE Trans. Antennas Propag., vol. 61, pp. 3850-3858, July 2013.

[152] R. Schoenen and H. Yanikomeroglu, "User-in-the-loop: Spatial and temporal demand shaping for sustainable wireless networks," IEEE Commun. Mag., vol. 52, pp. 196-203, Feb. 2014.

[153] D. S. Nunes, P. Zhang, and J. S. Silva, "A survey on human-in-the-loop applications towards an internet of all," IEEE Commun. Surveys Tuts., vol. 17, pp. 944-965, Feb. 2015.

[154] L. G. Jaimes, I. J. Vergara-Laurens, and A. Raij, "A survey of incentive techniques for mobile crowd sensing," IEEE Internet of Things Journal, vol. 2, pp. 370-380, Oct. 2015.

[155] S. Sen, G. Burtch, A. Gupta, and R. Rill, "Incentive design for ad-sponsored content: Results from a randomized trial," in IEEE Conference on Computer Communications Workshops (INFOCOM WKSHPS), pp. 826-831, May 2017.

[156] I. Bajaj, Y. H. Lee, and Y. Gong, "A spectrum trading scheme for licensed user incentives," IEEE Transactions on Communications, vol. 63, pp. 4026-4036, Nov. 2015.

[157] A. Özlü, K. Uludağ, and O. Korçak, "User provided network system with incentive mechanism," in 25th Signal Processing and Communications Applications Conference (SIU), pp. 1-4, May 2017.

[158] M. M. Khalili, L. Gao, J. Huang, and B. H. Khalaj, "Incentive design and market evolution of mobile user-provided networks," in IEEE Conference on Computer Communications Workshops (INFOCOM WKSHPS), pp. 498-503, Apr. 2015.

[159] M. Pouryazdan, B. Kantarci, T. Soyata, L. Foschini, and H. Song, "Quantifying user reputation scores, data trustworthiness, and user incentives in mobile crowdsensing," IEEE Access, vol. 5, pp. 1382-1397, Jan. 2017.

[160] N. Haderer, R. Rouvoy, and L. Seinturier, "A preliminary investigation of user incentives to leverage crowdsensing activities," in IEEE International Conference on Pervasive Computing and Communications Workshops (PERCOM Workshops), pp. 199-204, Mar. 2013.

[161] T. Giannetsos, S. Gisdakis, and P. Papadimitratos, "Trustworthy people-centric sensing: Privacy, security and user incentives road-map," in 13th Annual Mediterranean Ad Hoc Networking Workshop (MED-HOC-NET), pp. 39-46, June 2014.

[162] X. Sheng, J. Tang, X. Xiao, and G. Xue, "Sensing as a service: Challenges, solutions and future directions," IEEE Sensors Journal, vol. 13, pp. 3733-3741, Oct. 2013.

[163] R. K. Ganti, F. Ye, and H. Lei, "Mobile crowdsensing: Current state and future challenges," IEEE Communications Magazine, vol. 49, pp. 32-39, Nov. 2011. 
[164] Y. Li, B. Shen, J. Zhang, X. Gan, J. Wang, and X. Wang, "Offloading in HCNs: Congestion-aware network selection and user incentive design," IEEE Transactions on Wireless Communications, vol. 16, pp. 6479-6492, Oct. 2017.

[165] Z. Su, Q. Qi, Q. Xu, S. Guo, and X. Wang, "Incentive scheme for cyber physical social systems based on user behaviors," IEEE Transactions on Emerging Topics in Computing, Feb. 2017. Early Access.

[166] G. Iosifidis, L. Gao, J. Huang, and L. Tassiulas, "Incentive mechanisms for userprovided networks," IEEE Communications Magazine, vol. 52, pp. 20-27, Sept. 2014.

[167] F. B. Saghezchi, A. Radwan, A. Nascimento, and J. Rodriguez, "An incentive mechanism based on coalitional game for fair cooperation of mobile users in HANETs," in IEEE 17th International Workshop on Computer Aided Modeling and Design of Communication Links and Networks (CAMAD), pp. 378-382, Sept. 2012.

[168] S. Reddy, D. Estrin, M. Hansen, and M. Srivastava, "Examining micro-payments for participatory sensing data collections," in Proceedings of the 12th ACM International Conference on Ubiquitous Computing, pp. 33-36, Sept. 2010.

[169] M. Musthag, A. Raij, D. Ganesan, S. Kumar, and S. Shiffman, "Exploring microincentive strategies for participant compensation in high-burden studies," in Proceedings of the 13th ACM International Conference on Ubiquitous Computing, pp. 435-444, Sept. 2011.

[170] C. Dumitrescu, "On the design of a user-in-the-loop channel with application to emergency egress," arXiv:1508.03204 [cs], Aug. 2015. arXiv: 1508.03204.

[171] R. Schoenen and H. Yanikomeroglu, "User-in-the-loop: Spatial and temporal demand shaping for sustainable wireless networks," IEEE Communications Magazine, vol. 52, pp. 196-203, Feb. 2014.

[172] N. Sawyer and D. Smith, "U-BeAS: A Stackelberg game for device-to-device communications," arXiv:1603.00104 [cs], Feb. 2016. arXiv: 1603.00104.

[173] A. Ometov, "Enabling secure direct connectivity under intermittent cellular network assistance." https://trepo.tuni.fi/bitstream/handle/123456789/ 24260/ometov . pdf? sequence=3\&isAllowed=y, Sept. 2016. Accessed: 201911-26.

[174] S. Andreev, D. Moltchanov, O. Galinina, A. Pyattaev, A. Ometov, and Y. Koucheryavy, "Network-assisted device-to-device connectivity: Contemporary vision and open challenges," in Proceedings of 21st European Wireless Conference, pp. 1-8, May 2015.

[175] R. Barbosa, D. Nunes, A. Figueira, H. Aguiar, J. S. Silva, F. Gonzalez, C. Herrera, and S. Sinche, "An architecture for emotional smartphones in Internet of Things," in 2016 IEEE Ecuador Technical Chapters Meeting (ETCM), pp. 1-5, Oct. 2016.

[176] R. Schoenen, Z. Wang, H. Yanikomeroglu, N. G. Senarath, and D. Ngoc-Dung, "System and method for wireless load balancing," May 2 2017. US Patent 9,642,039. 
[177] R. Schoenen and H. Yanikomeroglu, "System and method for charging services using effective quanta units," Mar. 6 2018. US Patent 9,911,106.

[178] H. Shan, Y. Zhang, W. Zhuang, A. Huang, and Z. Zhang, "User behavior-aware scheduling based on time-frequency resource conversion," IEEE Trans. on Veh. Technol., vol. 66, pp. 8429-8444, Sept. 2017.

[179] C. Wang, S. H. Fang, H. C. Wu, S. M. Chiou, W. H. Kuo, and P. C. Lin, "Novel user-placement ushering mechanism to improve quality-of-service for femtocell networks," IEEE Systems Journal, vol. 12, pp. 1993-2004, June 2018.

[180] K. Suto, H. Nishiyama, and N. Kato, "Postdisaster user location maneuvering method for improving the QoE guaranteed service time in energy harvesting small cell networks," IEEE Transactions on Vehicular Technology, vol. 66, pp. 9410-9420, Oct. 2017.

[181] Z. Du, Y. Sun, W. Guo, Y. Xu, Q. Wu, and J. Zhang, "Data-driven deployment and cooperative self-organization in ultra-dense small cell networks," IEEE Access, vol. 6, pp. 22839-22848, Apr. 2018.

[182] W. Wang, L. Yang, Q. Zhang, and T. Jiang, "Configurations and diagnosis for ultradense heterogeneous networks: From empirical measurements to technical solutions," IEEE Network, vol. 32, pp. 138-145, June 2018.

[183] M. Mirahsan, G. Senarath, H. Farmanbar, N. D. Dao, and H. Yanikomeroglu, "Admission control of wireless virtual networks in HetHetNets," IEEE Transactions on Vehicular Technology, vol. 67, pp. 4565-4576, May 2018.

[184] L. S. Muppirisetty, S. Yiu, and H. Wymeersch, "LAPRA: Location-aware proactive resource allocation," in IEEE Global Communications Conference (GLOBECOM), pp. 1-6, Dec. 2016.

[185] R. Schoenen, G. Bulu, A. Mirtaheri, and H. Yanikomeroglu, "Green communications by demand shaping and user-in-the-loop tariff-based control," in IEEE Online Conference on Green Communications (GreenCom), pp. 64-69, Sept. 2011.

[186] A. T. Z. Kasgari, W. Saad, and M. Debbah, "Human-in-the-loop wireless communications: Machine learning and brain-aware resource management," IEEE Transactions on Communications, vol. 67, pp. 7727-7743, Nov. 2019.

[187] D. Yang, G. Xue, X. Fang, and J. Tang, "Crowdsourcing to smartphones: Incentive mechanism design for mobile phone sensing," in Proceedings of the 18th annual international conference on Mobile computing and networking, pp. 173-184, ACM, Aug. 2012.

[188] A. Chakeri and L. G. Jaimes, "An iterative incentive mechanism design for crowd sensing using best response dynamics," in IEEE International Conference on Communications (ICC), pp. 1-7, May 2017.

[189] W. Nan, B. Guo, S. Huangfu, Z. Yu, H. Chen, and X. Zhou, "A cross-space, multiinteraction-based dynamic incentive mechanism for mobile crowd sensing," in IEEE 11th Intl. Conf. on Ubiquitous Intelligence and Computing and IEEE 11th Intl. Conf. 
on Autonomic and Trusted Computing and IEEE 14th Intl. Conf. on Scalable Computing and Communications and Its Associated Workshops (UTC-ATC-ScalCom), pp. 179-186, Dec. 2014.

[190] M. Mirahsan, R. Schoenen, H. Yanikomeroglu, G. Senarath, and N. Dung-Dao, "User-in-the-loop for HetHetNets with backhaul capacity constraints," IEEE Wireless Communications, vol. 22, pp. 50-57, Oct. 2015.

[191] R. Schoenen, H. Yanikomeroglu, and B. Walke, "User in the loop: Mobility aware users substantially boost spectral efficiency of cellular OFDMA systems," IEEE Communications Letters, vol. 15, pp. 488-490, May 2011.

[192] R. Schoenen, G. Bulu, A. Mirtaheri, T. Beitelmal, and H. Yanikomeroglu, "Quantified user behavior in user-in-the-loop spatially and demand controlled cellular systems," in 18th European Wireless Conference, pp. 1-8, VDE, Apr. 2012.

[193] Z. Wang, L. Gao, and J. Huang, "Pricing competition of rollover data plan," in 16th International Symposium on Modeling and Optimization in Mobile, Ad Hoc, and Wireless Networks (WiOpt), pp. 1-8, May 2018.

[194] M. Murakami, R. Funaki, and J. Murata, "Design of incentive-based demand response programs using inverse optimization," in IEEE International Conference on Systems, Man, and Cybernetics (SMC), pp. 2754-2759, Oct. 2017.

[195] C. Evers, R. Kniewel, K. Geihs, and L. Schmidt, "The user in the loop: Enabling user participation for self-adaptive applications," Elsevier Future Generation Computer Systems, vol. 34, pp. 110-123, May 2014.

[196] Z. Wang, R. Schoenen, H. Yanikomeroglu, and M. St-Hilaire, "Load balancing in cellular networks with user-in-the-loop: A spatial traffic shaping approach," in IEEE International Conference on Communications (ICC), pp. 2638-2643, June 2015.

[197] N. Sawyer and D. Smith, "Dynamic Stackelberg user-in-the-loop repeated game for device-to-device communications," arXiv preprint arXiv:1603.00104, 2016.

[198] S. Boyd and L. Vandenberghe, Convex Optimization. Cambridge University Press, 2004.

[199] C. D'Ambrosio, A. Lodi, and S. Martello, "Piecewise linear approximation of functions of two variables in MILP models," Operations Research Letters, vol. 38, pp. 39-46, Jan. 2010.

[200] J. Kallrath, Algebraic Modeling Systems: Modeling and solving real world optimization problems, vol. 104. Springer Science \& Business Media, 2012.

[201] L. A. DaSilva, "Pricing for qos-enabled networks: A survey," IEEE Communications Surveys Tutorials, vol. 3, no. 2, pp. 2-8, 2000.

[202] P. Demestichas, A. Georgakopoulos, D. Karvounas, K. Tsagkaris, V. Stavroulaki, J. Lu, C. Xiong, and J. Yao, "5G on the horizon: Key challenges for the radio-access network," IEEE Veh. Technol. Mag., vol. 8, pp. 47-53, Sept. 2013. 
[203] S. Chandrasekharan, K. Gomez, A. Al-Hourani, S. Kandeepan, T. Rasheed, L. Goratti, L. Reynaud, D. Grace, I. Bucaille, T. Wirth, et al., "Designing and implementing future aerial communication networks," IEEE Communications Magazine, vol. 54, pp. 26-34, May 2016.

[204] Y. Zeng, R. Zhang, and T. J. Lim, "Throughput maximization for UAV-enabled mobile relaying systems," IEEE Trans. on Commun., vol. 64, pp. 4983-4996, Sept. 2016.

[205] A. Bradai, K. Singh, T. Ahmed, and T. Rasheed, "Cellular software defined networking: A framework," IEEE Commun. Mag., vol. 53, pp. 36-43, June 2015.

[206] C. Liang and F. Yu, "Wireless virtualization for next generation mobile cellular networks," IEEE Wireless Communications, vol. 22, pp. 61-69, Feb. 2015.

[207] K. Dalamagkidis, Handbook of Unmanned Aerial Vehicles, ch. Classification of UAVs, pp. 83-91. Springer Netherlands, 2015.

[208] "Scenarios, requirements and KPIs for 5G mobile and wireless system," report, METIS 2020, 2013. Accessed: 2020-02-18.

[209] H. Kaushal and G. Kaddoum, "Free space optical communication: Challenges and mitigation techniques," arXiv:1506.04836 [cs, math], June 2015. Accessed: 201911-23.

[210] U. Siddique, H. Tabassum, E. Hossain, and D. I. Kim, "Wireless backhauling of 5G small cells: Challenges and solution approaches," IEEE Wireless Communications, vol. 22, pp. 22-31, Oct. 2015.

[211] X. Zhou, Z. Zhao, R. Li, Y. Zhou, T. Chen, Z. Niu, and H. Zhang, "Toward 5G: When explosive bursts meet soft cloud," IEEE Network, vol. 28, pp. 12-17, Nov. 2014.

[212] V. Yazici, U. C. Kozat, and M. O. Sunay, "A new control plane for 5G network architecture with a case study on unified handoff, mobility, and routing management," IEEE Communications Magazine, vol. 52, pp. 76-85, Nov. 2014.

[213] S. Sezer, S. Scott-Hayward, P. Chouhan, B. Fraser, D. Lake, J. Finnegan, N. Viljoen, M. Miller, and N. Rao, "Are we ready for SDN? Implementation challenges for software-defined networks," IEEE Commun. Mag., vol. 51, pp. 36-43, July 2013.

[214] S. Zaidi, S. Affes, U. Vilaipornsawai, L. Zhang, and P. Zhu, "Wireless access virtualization strategies for future user-centric 5G networks," in IEEE Globecom Workshops (GC Wkshps), pp. 1-7, Dec.

[215] K. Zarifi, H. Baligh, J. Ma, M. Salem, and A. Maaref, "Radio access virtualization: Cell follows user," in IEEE 25th Annual International Symposium on Personal, Indoor, and Mobile Radio Communication (PIMRC), pp. 1381-1385, Sept. 2014.

[216] J. Zhang, L. Lu, Y. Sun, Y. Chen, J. Liang, J. Liu, H. Yang, S. Xing, Y. Wu, J. Ma, I. B. F. Murias, and F. J. L. Hernando, "PoC of SCMA-based uplink grant-free transmission in UCNC for 5G," IEEE J. Sel. Areas Commun., vol. 35, pp. 1353-1362, June 2017. 
[217] S. Bassoy, M. Jaber, M. A. Imran, and P. Xiao, "Load aware self-organising usercentric dynamic CoMP clustering for 5G networks," IEEE Access, vol. 4, pp. 28952906, May 2016.

[218] A. Maaref, J. Ma, M. Salem, H. Baligh, and K. Zarin, "Device-centric radio access virtualization for 5G networks," in IEEE Globecom Workshops (GC Wkshps), pp. 887-893, Dec. 2014.

[219] "Guidelines for evaluation of radio interface technologies for IMT-advanced," Recommendation ITU-R M.2135-1, International Telecommunication Union Radiocommunication Sector (ITU-R), Dec. 2009.

[220] K. Shen and W. Yu, "Fractional programming for communication systems-Part I: Power control and beamforming," IEEE Transactions on Signal Processing, vol. 66, no. 10, pp. 2616-2630, 2018.

[221] K. Shen and W. Yu, "Fractional programming for communication systems-Part II: Uplink scheduling via matching," IEEE Transactions on Signal Processing, vol. 66, no. 10, pp. 2631-2644, 2018.

[222] D. Athukoralage, I. Guvenc, W. Saad, and M. Bennis, "Regret based learning for UAV assisted LTE-U/WiFi public safety networks," in IEEE Global Communications Conference (GLOBECOM), pp. 1-7, Dec. 2016.

[223] R. S. Sutton and A. G. Barto, Reinforcement Learning: An Introduction. Cambridge, MA: MIT Press, 2011.

[224] H. R. Tizhoosh, "Reinforcement learning based on actions and opposite actions," in International conference on artificial intelligence and machine learning, vol. 414, 2005.

[225] Chi-Hyon Oh, T. Nakashima, and H. Ishibuchi, "Initialization of Q-values by fuzzy rules for accelerating Q-learning," in IEEE International Joint Conference on Neural Networks, IEEE World Congress on Computational Intelligence, vol. 3, pp. 20512056, 1998.

[226] R. S. Sutton, "Learning to predict by the methods of temporal differences," Machine learning, vol. 3, no. 1, pp. 9-44, 1988.

[227] P. Dayan, "The convergence of TD $(\lambda)$ for general $\lambda$," Machine learning, vol. 8, no. 3-4, pp. 341-362, 1992.

[228] J. Luo, Q. Chen, F. R. Yu, and L. Tang, "Blockchain-enabled software-defined industrial Internet of Things with deep reinforcement learning," IEEE Internet of Things Journal, 2020. [Early Access].

[229] T. Tomic, K. Schmid, P. Lutz, A. Domel, M. Kassecker, E. Mair, I. L. Grixa, F. Ruess, M. Suppa, and D. Burschka, "Toward a fully autonomous UAV: Research platform for indoor and outdoor urban search and rescue," IEEE Robot. Autom. Mag., vol. 19, pp. 46-56, Sept. 2012. 
[230] "Mobile-enabled unmanned aircraft - How mobile networks can support unmanned aircraft operations," tech. rep., GSMA, Feb. 2018. https://wwW.gsma.com/iot/wp-content/uploads/2018/02/ Mobile-Enabled-Unmanned-Aircraft-web .pdf Accessed: 2020-02-18.

[231] H. Zhang, S. Vrzic, G. Senarath, N. D. Do, H. Farmanbar, J. Rao, C. Peng, and H. Zhuang, "5G wireless network: MyNET and SONAC," IEEE Network, vol. 29, pp. 14-23, July 2015.

[232] S.-Y. Lien, S.-L. Shieh, Y. Huang, B. Su, Y.-L. Hsu, and H.-Y. Wei, "5G New radio: Waveform, frame structure, multiple access, and initial access," IEEE Commun. Mag., vol. 55, pp. 64-71, June 2017.

[233] “Overview on IAB,” Tech. Rep. R3-181998, 3GPP RAN WG-3, Apr. 2018.

[234] ETSI Zero Touch Network and Service Management, "Zero-touch network and service management - Operators' view on the necessity of automation in end-to-end network and service management, and operation," Dec. 2017. https://portal. etsi . org/TBSiteMap/ZSM/OperatorWhitePaper Accessed: 2018-04-04.

[235] "Documentation of requirements and KPIs and definition of suitable evaluation criteria," Tech. Rep. Deliverable D6.1, 5G Mobile Network Architecture, Sept. 2017.

[236] M. Stojanovic, Z. Bogdanovic, D. Barac, M. Radenkovic, and J. MihajlovicMilicevic, "The role of AI in the transformation of mobile operators," in International Conference on Artificial Intelligence: Applications and Innovations (ICAIAI), pp. 90-904, Sep. 2019.

[237] J. Kang, Z. Xiong, D. Niyato, Y. Zou, Y. Zhang, and M. Guizani, "Reliable federated learning for mobile networks," IEEE Wireless Communications, pp. 1-9, Feb. 2020. Early Access.

[238] X. Wang, Y. Han, V. C. M. Leung, D. Niyato, X. Yan, and X. Chen, "Convergence of edge computing and deep learning: A comprehensive survey," IEEE Communications Surveys $\mathcal{E}$ Tutorials, 2020. Early Access.

[239] Y. Liu, F. R. Yu, X. Li, H. Ji, and V. C. M. Leung, "Blockchain and machine learning for communications and networking systems," IEEE Communications Surveys $\mathcal{E}$ Tutorials, 2020. Early Access.

[240] D. V. Medhane, A. K. Sangaiah, M. S. Hossain, G. Muhammad, and J. Wang, "Blockchain-enabled distributed security framework for next generation IoT: An edge-cloud and software defined network integrated approach," IEEE Internet of Things Journal, 2020. Early Access.

[241] "Jeff Bezos and Elon Musk to compete for 5G backhaul business." https://www.lightreading.com/mobile/backhaul/ jeff-bezos-and-elon-musk-to-compete-for-5g-backhaul-business / d/d-id/752654. Accessed: 2020-03-14.

[242] N. Rupasinghe, Y. Yapıc1, I. Güvenç, M. Ghosh, and Y. Kakishima, "Angle feedback for NOMA transmission in mmWave drone networks," IEEE Journal of Selected Topics in Signal Processing, vol. 13, pp. 628-643, June 2019. 